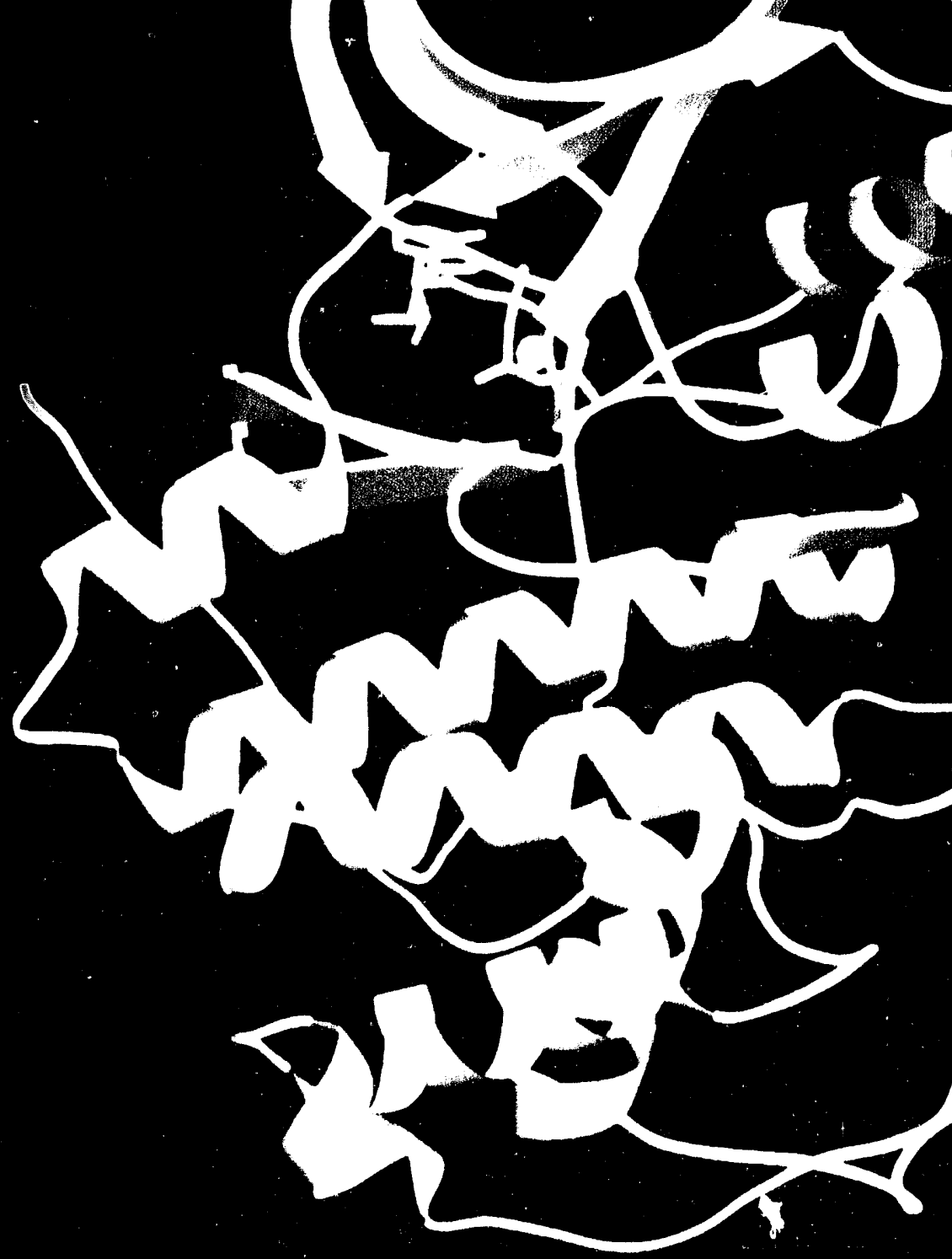


Cover caption: Scientists at LBL, in close partnership with UC Berkeley and UC San Francisco have solved the three-dimensional structure of a key protein, cyclin-dependent protein kinase $(C K D-2)$, that dispatches one of the last signals in the chain of events leading to the division of a cell. The importance of the achievement lies in the fact that uncontrolled cell division is a hallmark of cancer. This is the first three-dimensional structure determined for proteins controlling cell division.

The figure shows three-dimensional structure of the human CDK2 in complex with magnesium and adenosine triphosphate (ATP). The red/pink color represents the a helix while the green/ blue colors are the $b$-strands of the protein. They are connected by the loops shown in yellow. The pink ball indicates the magnesium atom, while the orange line segments are the ATP. The structure consists of two domains; one is primarily composed of five b-strands and the other of six a helices. The transfer of a phosphate group from ATP to the target proteins (necessary for cell division) by this complex signals the commitment of the cells to divide.

This research contributed to the structural biology program of $D O E^{\prime}$ ' Office of Health and Environmental Research. The structure was determined using $x$-rays from synchrotron radiation sources. DOE's biosciences capability will be greatly strengthened at LBL's Advanced Light Source.

\title{
DISCLAIMER
}

\begin{abstract}
This document was prepared as an account of work sponsored by the United States Government. Neither the United States Government nor any agency thereof, nor The Regents of the University of California, nor any of their employees, makes any warranty, express or implied, or assumes any legal liability or responsibility for the accuracy, completeness, or usefulness of any information, apparatus, product, or process disclosed, or represents that its use would not infringe privately owned rights. Reference herein to any specific commercial product, process, or service by its trade name, trademark, manufacturer, or otherwise, does not necessarily constitute or imply its endorsement, recommendation, or favoring by the United States Government or any agency thereof, or The Regents of the University of California. The views and opinions of authors expressed herein do not necessarily state or reflect those of the United States Government or any agency thereof or The Regents of the University of California and shall not be used for advertising or product endorsement purposes.
\end{abstract}

Lawrence Berkeley Laboratory is an equal opportunity employer. 


\section{LAWRENCE BERKELEY LABORATORY INSTITUTIONAL PLAN FY 1994-1999}

September 1993

Lawrence Berkeley Laboratory

University of California

Berkeley, California 94720

\section{MASTER}

Prepared for the U.S. Department of Energy under Contract No. DE-AC03-76SF00098 


\section{PREFACE}

The Institutional Plan provides an overview of the Lawrence Berkeley Laboratory mission, strategic plan, scientific initiatives, research programs, environment and safety program plans, educational and technology transfer efforts, human resources, and facilities needs. For FY 1994-1999 the Institutional Plan reflects significant revisions based on the Laboratory's strategic planning process.

The Strategic Plan section identifies long-range conditions that will influence the Laboratory, as well as potential research trends and management implications. The Initiatives section identifies potential new research programs that represent major long-term opportunities for the Laboratory, and the resources required for their implementation. The Scientific and Technical Programs section summarizes current programs and potential changes in research program activity. The Environment, Safety, and Health section describes the management systems and prograrns underway at the Laboratory to protect the environment, the public, and the employees. The Technology Transfer and Education programs section describes current and planned programs to enhance the nation's scientific literacy and human infrastructure and to improve economic competitiveness. The Human Resources section identifies LBL staff diversity and development programs. The section on Site and Facilities discusses resources required to sustain and improve the physical plant and its equipment. The new section on Information Resources reflects the importance of computing and communication resources to the Laboratory. The Resource Projections are estimates of required budgetary authority for the Laboratory's ongoing research programs.

The Institutional Plan is a management report for integration with the Department of Energy's strategic planning activities, developed through an annual planning process. The plan identifies technical and administrative directions in the context of the national energy policy and research needs and the Department of Energy's program planning initiatives. Preparation of the plan is coordinated by the Office for Planning and Deveiopment from information contributed by the Laboratory's scientific and support divisions. 


\section{TABLE OF CONTENTS}

Preface iii

1. Director's Statement 1-1

2. The Laboratory Mission $2-1$

Core Competencies and Foundations $2-1$

Division Strengths $2-3$

3. Laboratory Strategic Plan 3-1

LBL's Vision and Strategic Objectives 3-1

Situation Analysis $\quad 3-2$

LBL's Strategy 3-7

Strategic Issues $\quad 3-10$

4. Initiatives 4-1

Basic Energy Sciences $\quad 4-2$

High Energy and Nuclear Physics 4-11

Office of Health and Environmental Research 4-16

Energy Efficiency and Renewable Energy 4-21

Domestic and Energy Policy $\quad 4-22$

Fusion Energy 4-23

Environmental Restoration and Waste Management $\quad 4-25$

Work for Cithers $\quad$ 4-26

5. Scientific and Technical Programs 5-1

Office of Energy Research 5-1

Energy Efficiency and Renewable Energy $\quad 5-33$

Office of Fossil Energy $\quad 5-36$

Environmental Restoration and Waste Management 5-37

Environment, Safety, and Health $\quad 5-37$

Other DOE Programs $\quad$ 5-38

Work for Others $\quad$ 5-39

Laboratory Directed Research and Development $\quad 5-44$ 
6. Environment, Safety, and Health Management

ES\&H Goals and Objectives

Current Conditions

ES\&H Policies, Organization, and Management

ES\&H Plans and Initiatives

Environmental Restoration and Waste Management

7. Technology Transfer and Science Education

Technology Transfer

Science, Mathematics, and Engineering Education

8. Human Resources

Laboratory Personnel

8-1

Personnel Development Programs

Work Force Diversity

Affirmative Action 1993

9. Site and Facilities

Site Development Plans

9-1

Site Description and Status

9-2

Facilities Plans and Options

General Purpose Facilities Plans

Programmatic Facilities Plans

Facilities Decommissioning Plan

Facilities Resources Requirements

10. Information Resources Management

Goals and Objectives

10-1

Current Situation

Strategies

10-6

Resources and Initiatives 


\section{DiRECTOR'S STATEMENT}

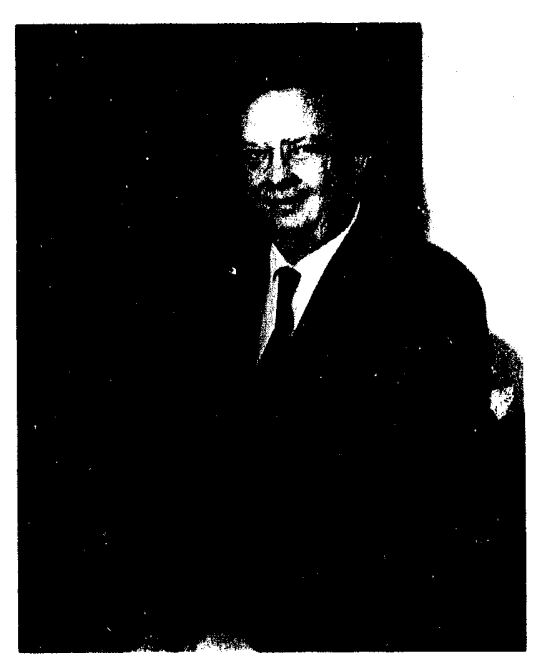

Charles V. Shank, LBL Director

\begin{abstract}
Vision 2000 is our strategic blueprint for the future. It focuses on basic research as our primary endeavor and establishes a commitment to reach out and forge new partnerships with industry, academia, and other national laboratories, to develop our resources and our people to create value for the economy, to enhance education, and to contribute to the community.
\end{abstract}

For more than 60 years Lawrence Berkeley Laboratory (LBL) has maintained its strong tradition of outstanding research. Established as a particle physics accelerator facility, the Laboratory has undergone dramatic changes since its early years, diversifying into a multiprogram institution that engages not only in accelerator research, but also in research in the chemical sciences, earth sciences, advanced materials, energy, and the biosciences. One of LBL's greatest strengths through the years has been its ability to be at the frontier of changing scientific priorities. This year's Institutional Plan takes us to the threshold of the 21 st century, where once again the Laboratory is addressing broad new challenges.

Although the energy mission of the Department of Energy is sustained, the role of the national laboratories is undergoing fundamental change. With the end of the cold war, pressing national budget constraints, and a new administration, new federal priorities have emerged. Economic growth, competitiveness in the global market, and the preeminence of science and engineering education have become driving concerns for the government, private industry, and the public. This year, under the direction of the new Secretary of Energy, Hazel O'Leary, the Department of Energy itself was restructured to mirror the priorities of a changed world. The goal of the restructuring is to decrease emphasis on defense programs and increase the commitment of resources to promote energy efficiency, technology partnerships, and environmental restoration, with the ultimate goal of enhancing 
U.S. economic and technological competitiveness. I believe that the national laboratories will play a critical role in meeting these new challenges. LBL is committed to developing new partnerships that will move our research more quickly into application, whether those applications are for the marketplace or the advancement of research in the scientific community.

In 1992 I initiated a major strategic planning effort at LBL. The purpose of this effort is to define the Laboratory's major objectives as it moves toward the new century; to provide LBL, its employees, its partners, and the Department of Energy with a clear statement of our plans and goals for the future.

Vision 2000, which is referenced throughout this Institutional Plan, is our strategic blueprint for the future. It affirms basic research as our foundation and establishes a commitment to reach out and forge partnerships with industry, academia, and other national laboratories, to develop our resources and our people to create value for the economy, to enhance education, and to contribute to the community. To address these issues, and others raised in the Laboratory's strategic planning effort, specific task forces were formed to study several vital areas: core competencies, national research needs, forging partnerships, creating an LBL that works, developing our facilities, and committing to our people. The reports of these task forces will become the basis for institutional changes to make our vision a reality. Some of these changes have already been accomplished as L.BL sustains and builds on its foundation of scientific excellence.

LBL plans to strengthen its user facilities already serving national research needs. The Advanced Light Source has been completed on schedule, within budget, and with performance exceeding project specifications. This begins an era of scientific programs that will be at the forefront of research in materials, surfaces, interfaces, chemical dynamics, and biosciences, with partners drawn from the national scientific community. Outstanding support for all our users is a key element of our strategic plan. Consistent with this, completion of the Advanced Light Source Beamlines Initiative is a crucial element of the Laboratory's strategic planning. The Laboratory's Structural Biology Initiative will add user capabilities for studies in biological microscopy and spectroscopy. The upgrade of electron microscopes at the National Center for Electron Microscopy with a OneAngstrom Microscope and a Magnetic Materials Microscope will enable our users to continue working at the world's leading facility.

LBL's commitment to partnering arises from its long-standing collaboration with the University of California at Berkeley. The fruits of this collaboration have been more than world renowned science and the education of the next generation of scientists. LBL has also learned the institutional lessons required to sustain such mutually beneficial relations over a sustained period-understanding and integrating the broad goals of one's partners into the goals of one's own institution. Our location in one of the country's major high technology centers, the San Francisco Bay Area, has also lent itself to increasingly stronger links with a wide variety of industries, and therefore a stronger role in technology transfer activities. We hope to build on those activities with more Cooperative Research and Development Agreements and technology transfer initiatives. Beyond that, the Laboratory's Center for Science and Engineering Education at L.BL has been extraordinarily effective at forming links with all levels of educational institutions, industry partners, and the wider community at large. It continues to develop new methods for outreach and new opportunities for sharing 
the resources and facilities of our national laboratories to promote excellence in education.

LBL continues to emphasize the value of scientific partnerships through such initiatives as the Combustion Dynamics Initiative. This program will combine the capabilities of two national laboratories, Sandia and LBL, as well as the educational ties of LBL with the University of California to research the characteristics and potential control of fossil combustion and emission. Support for the molecular scale design and synthesis of advanced materials will enable industry, academia, and the national laboratories to make advances in materials technology. These LBL programs will support the Department of Energy's efforts to increase energy efficiency, protect the environment, and promote economic competitiveness, as well as affect new energy technologies. In the life sciences, LBL is working in close collaboration with the University of California campus as well as industrial partners, as it continues research on the human genome. The Human Genome Laboratory will provide essential facilities as we work to meet the national scientific and technical goals involved in this effort to map and sequence the human chromosomes.

LBL will maintain its historic strength in high-energy and nuclear physics, providing leadership for the Superconducting Super Collider and the STAR detector at RHIC. Building on a core competency, the Laboratory will continue to utilize its accelerator and detector capabilities to serve the scientific community. For example, LBL's heavy-ion fusion accelerator research is leading the nation's inertial confinement fusion energy program, which may provide long-term electricity supply during the middle of the 21 st century.

LBL's continuing commitment to its people has been reflected in basic institutional changes that were made earlier this year. A new Office of Work Force Diversity was established to ensure a cultural ethic that fully respects diversity and encourages excellence. In addition, we have created a new management structure to enhance internal and external communications, program developments, and opportunities for supporting leadership. Our commitment to the environment continues to be extremely important in our day-to-day operations. We have incorporated major changes in our response to environmental and safety concerns through a continuous improvement program that ensures that our work is carried out with full regard for the environment, and the health and safety of our employees and neighbors.

As LBL charts its course into the 21 st century, we will continue to refine our strategic objectives, and to articulate clearly this institution's contribution to the missions of the Department of Energy and its partners. We are strengthening our partnerships with industry, other laboratories, and educational institutions to better serve both community and national needs. Finally, we will continue to engage in fundamental research at the scientific frontiers, which is the key to attracting and retaining our outstanding staff, the foundation of LBL's excellence. Our ultimate goal is to see our people and our technological resources continue to serve as a unique and valuable resource for the Department of Energy and the nation.

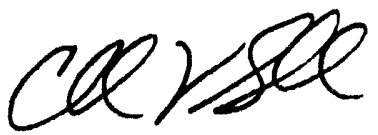

Charles V. Shank

Director 


\section{THE LABORATORY MISSION}

The Lawrence Berkeley Laboratory is a multiprogram national research facility operated by the University of California for the Department of Energy. Its fundamental mission is to provide national scientific leadership and technological innovation to support the DOE's objectives. LBL's mission is divided into four distinct goals:

- To perform leading multidisciplinary research in the energy sciences, general sciences, and biosciences in a manner that ensures employee and public safety and protection of the environment.

- To develop and operate unique national experimental facilities that are available to qualified investigators: The Advanced Light Source, National Center for Electron Microscopy, 88-Inch Cyclotron, and National Tritium Labeling Facility.

- To educate and train future generations of scientists and engineers to promote national science and education goals.

- To transfer knowledge and technological innovations and foster productive relationships between LBL research programs, universities, and industry to promote national economic competitiveness.

\section{CORE COMPETENCIES AND FOUNDATIONS}

The ability of the Laboratory to advance its mission depends upon a small number of "core competencies." Recognizing our core competencies is a necessary step in deciding where we as a laboratory can make the strongest and most important contributions to the changing missions of the agencies we serve. 
Core competency is defined as the integration of research disciplines, personnel, skills, technologies, and facilities which produce results of value to our sponsors. These results are in the form of basic research, applied research, technical advances, and research training. Core competencies can be applied to rapidly changing national needs and new research problems while, at the same time, undergoing evolution themselves.

Beyond our core competencies in specific technical areas, there are also several key capabilities that are integrated into, or underlie, many of the core competencies. We refer to these as "foundations" in order to distinguish them from the core competencies.

LBL has identified six core competencies and four foundations. They are listed below.

\section{Core Competencies}

- Bioscience and Biotechnology: Structural biology; genome research; bioinstrumentation; molecular cytogenetics; medical imaging; biology of human diseases; biomolecular design.

- Particle and Photon Beams: Analysis and design of accelerators; beam dynamics; high-brightness, ion, and photon sources; advanced magnet design and R\&D; high-frequency rf technology; $x$-ray optics and lithography; induction linacs and neutral beams for fusion energy.

- Characterization and Synthesis of Materials: Advanced spectroscopies and microscopies based on photons, electrons, and scanning probes; ceramics; alloys; heterostructures; superconducting, magnetic, and atomically structured materials; bioorganic synthesis.

- Advanced Technologies for Energy Supply and Energy Efficiency: Subsurface resources and processes; building technologies; electrochemistry; fossil fuel technologies; energy analysis.

- Chemical Dynamics, Catalysis, and Surface Science: Reaction dynamics; photochemistry of molecules and free radicals; surface structures and functions; heterogeneous, homogeneous, and enzymatic catalysis.

- Advanced Detector Systems: Major detectors for high-energy physics, nuclear science, and astrophysics; scientific conception and project leadership; advances in particle and photon detection; implementation of new concepts in detector technology.

In addition, there is an emerging core competency in Environmental Assessment and Remediation. This includes advanced instrumentation and methods for environmental characterization and monitoring; human health and ecological risk assessment; indoor air quality; subsurface remediation of contuminants; geologic isolation of high-level nuclear waste; and actinide chemistry.

\section{Foundations}

- National Research Facilities: Advanced Light Source; National Center for Electron Microscopy; 88-Inch Cyclotron; National Tritium Labeling Facility. 
- Computation and Information Management: High-speed networking and distributed computing; processing and analysis of scientific images: data-acquisition and analysis systems; scientific information systems; datahase technology.

- Engineering Design and Fabrication Technologies: Custom integrated circuits; integrated accelerator systems; superconducting magnet assemblies; insertion devices for synchrotron radiation; large-volume semiconductor detector technology; laboratory automation; advanced CAD/CAM facilities for large systems; facilities for materials processing and fabrication.

- Education of Future Scientists and Engineers: Undergraduate, graduate, postdoctoral, and faculty involvement in scientific arid engineering research through close ties with the University of California system; educational programs for elementary schools, high schools, and colleges.

\section{DIVISION STRENGTHS}

LBL benefits from its close working relationship with the University of California at Berkeley, as well as other universities, laboratories, and industrial institutions. The Laboratory is structured, as indicated in the organization chart, to integrate these relationships and its mission in the most effective way possible. The core research strengths of each of the LBL divisions are as follows:

\section{Energy Sciences Divisions}

- Chemical Sciences--chemical physics and the dynamics of chemical reactions; structure and reactivity of transient species; electron spectroscopy; surface chemistry and catalysis; chemistry of the actinide elements and their relationship to environmental issues; atomic physics.

- Earth Sciences--structure, composition, and dynamics of the earth's subsurface; geophysical imaging methods; chemical and physical transport in geologic systems; isotopic geochemistry; physicochemical process investigations.

- Energy and Environment-building energy efficiency; environmental effects of technology; energy storage and distribution; fossil-energy conversion; industry and utility energy use; national and international energy policy studies.

- Materials Sciences-advanced ceramic, metallic, polymeric, magnetic, biological and semi- and superconducting materials for catalytic, electronic, optical, magnetic, structural, and specialty applications; exploration of low-dimensional materials; development and use of instrumentation including spectroscopies, electron microscopy, $x$-ray optics, nuclear magnetic resonance, and analytical tools for ultrafast processes and surface analysis. 


\section{General Sciences Divisions}

- Accelerator and Fusion Research-fundamental accelerator physics research; accelerator design and operation; advanced accelerator technology development; arcelerator and ion-source research for heavy-ion fusion and magnetic fusion; construction and operation of the Advanced Light Source.

- Nuclear Science-relativistic heavy-ion physics; medium- and lowenergy nuclear physics; nuclear structure; nuclear theory; nuclear astrophysics; weak interactions; nuclear chemistry; studies of transuranium elements; nuclear-data evaluation; detector development; and operation of the 88-Inch Cyclotron.

- Physics-experimental and theoretical particle physics; advanced detector development; particle database for the high-energy physics community; astrophysics; applied mathematics.

\section{Biosciences Divisions}

- Life Sciences-gene expression; molecular genetics and human genome studies; cellular differentiation and carcinogenesis; hematopoiesis; macromolecular structure; DNA repair and recombination; diagnostic and functional imaging; and radiation biology.

- Structural Biology - structural and molecular biology of nucleic acids and proteins; genetics and mechanisms of photosynthesis; photochemistry; mechanisms of mutagenesis.

\section{Resources and Operations Divisions}

- Engineering-engineering design, planning, and concept development; advanced accelerator components; electronic and mechanical instrumentation; scientific applications software development; laboratory automation; fabrication of detectors and experimental systems; shops and technical support for scientific programs and research facilities.

- Environment, Health and Safety-technical support for safety and environmental protection; radiation associated with accelerator technology; advanced dosimeters; dispersion of radionuclides; waste management.

- Information and Computing Sciences--advanced software engineering; information management; scientific imaging and visualization tools; computation tools for the human genome project; biostatistics; agile manufacturing. 


\section{LAWRENCE BERKELEY LABORATORY • UNIVERSITY OF CALIFORNIA}

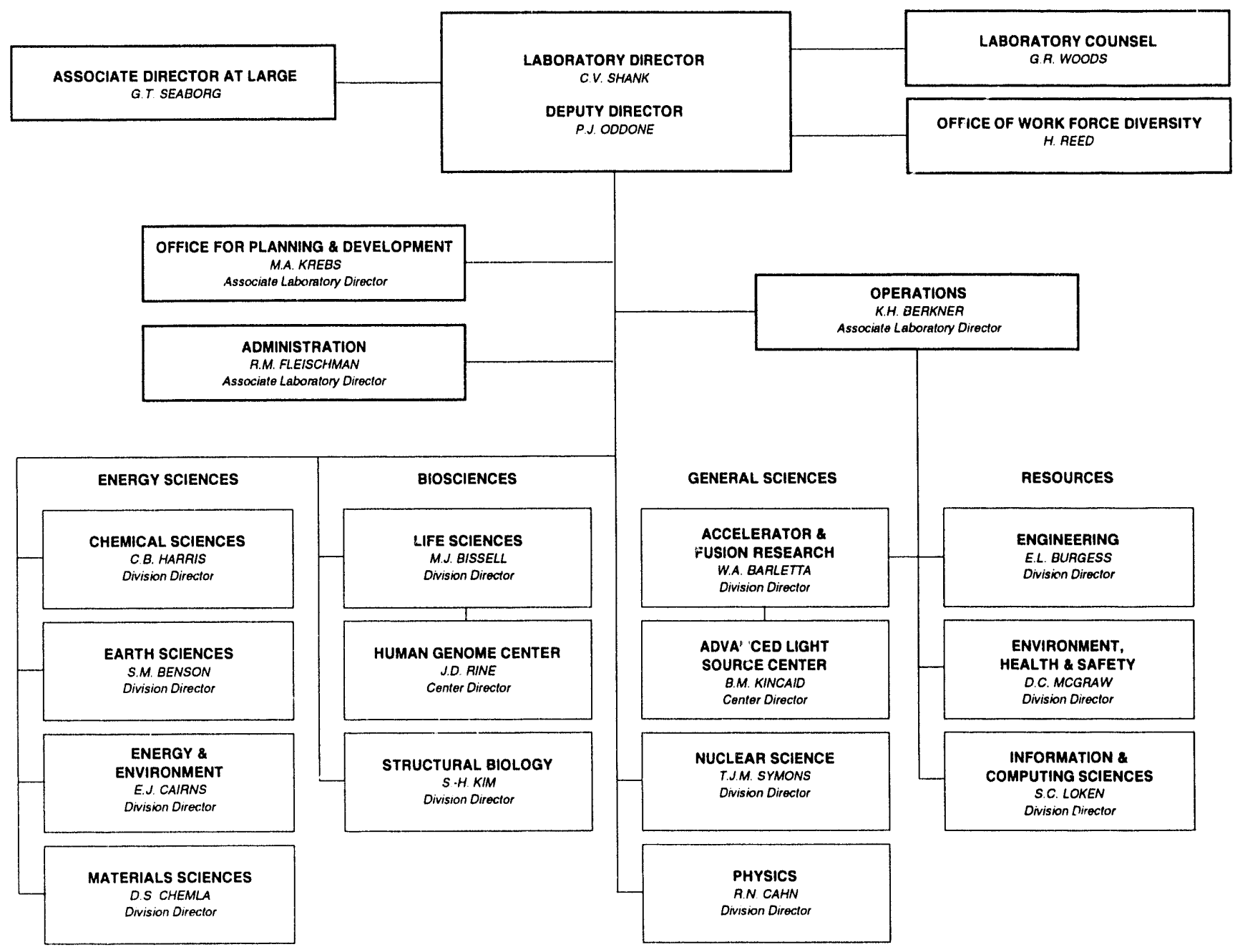

$L B L$ organization chart. 


\section{LABORATORY STRATEGIC PLAN}

\section{LBL'S VISION AND STRATEGIC OBJECTIVES}

LBL has undertaken a comprehensive strategic planning effort to define our vision, core competencies, strategic objectives, and implementary actions. This has included assessing our programs and operating context, defining specific issues confronting our future, and addressing both $L B L$ and DOE program objectives. At the same time, $L B L$ has been working with $D O E$ and other national laboratories to define mutual R\&D roles to support the DOE mission, planning, and quality management initiatives.

Since all employees and the research community have a stake in the Laboratory's future, LBL has established mechanisms for broad involvement of stakeholders in developing the strategic plan. Specific task forces have been established to define issues and objectives and to develop recommendations in the following areas: national research needs, distinctive competencies, scientific initiatives, partnerships, commitment to our people, and making an LBL that works. Through the work of these task forces, the Laboratory has begun to formulate a long-term strategy that will integrate all of the fundamental elements of its operations.

The strategy outlined in this section is a response to significant changes in the R\&D environment that have affected the Laboratory's research priorities and the allocation of its resources. As a national laboratory, LBL is committed to addressing the critical research and technical issues facing the nation. We have identified these issues and proposed a direction that will maintain the Laboratory's position of scientific and engineering leadership well into the 21 st century.

LBL's Vision 2000 establishes the overarching goals of the Laboratory's strategic planning effort. It focuses on the DOE as our primary sponsor, but also establishes a commitment to reaching out and forging new partnerships with industry, academia, and other national laboratories to create value for 
the economy, enhance education, and contribute to the community. The four major goals of Vision 2000 are as follows:

Distinguish ourselves as a premier DOE multiprogram national laboratory by performing research of the highest scientific quality. Build on our educational and technical resources to gain a competitive advantage for addressing problems of national significance and advancing the mission of the DOE.

Create value for the economy, enhance education, and contribute to the community through partnerships with industry, universities, and other laboratories.

Make LBL the location of choice for facilities and programs. Our operational, administrative, and technical resources will integrate seamlessly with the research and engineering programs to make an LBL that works. All of our activities will be conducted with full regard for the environment, health, and safet;".

Commit to developing our people to their fullest potential. We value and seek diversity in our work force. We will create an environment that respects the individual, encourages leadership, stimulates innovation, fosters integrity, and demands excellence.

To achieve these goals, we must understand the context of our research programs within the framework of DOE, other national laboratories, and the nation, and must incorporate this information into our strategic planning effort.

\section{SITUATION ANALYSIS}

As a DOE Laboratory, L.BL is dedicated to multiprogram energy research that addresses vital energy, economic, and educational interests. This mission has not changed; however, the world has entered an era fundamentally different from the one that motivated the establishment of the national laboratories. Pressing economic conditions in concert with the end of the Cold War have caused a reevaluation of national research priorities within the larger world community. U.S. global economic competitiveness and the preeminence of U.S. science and engineering have emerged as important Federal goals, along with new imperatives for improving government and addressing health and environmental concerns. As a result, public perception of the role of science and the basis for supporting science at national laboratories is changing, and the Laboratory must find new ways to demonstrate the benefits of its research to society. National laboratories will be called upon by many stakeholders for leadership in improving national energy efficiency, providing an improved basis for securing adequate domestic energy supplies and protecting health and the environment, as well as developing the foundations of scientific and engineering research.

\section{National Research Needs}

A major objective of the LBL strategic planning effort is to match the Laboratory's core competencies with national research needs. This will not only enhance our ability to meet DOE's national goals, but will also ensure 
the vitality of the Laboratory and its research. To better understand the new R\&D environment, LBL's task force undertook a study to identify national research needs that are important to our strategic planning. The task force identified three major categories of national need: $R \& D$ needs of a technological society, R\&D to secure our energy and environmental future, and scientific foundations.

\section{R\&D Needs of a Technological Society}

- Materials for technological applications. These efforts are broadly aimed at the development of superconducting, catalytic, polymeric, magnetic, biological, and tribiological materials and materials for electronic applications that have long-term economic benefit. Crosscutting research efforts include those aimed at developing nanometerscale artificially structured materials; materials for harsh chemical environments and high-radiation applications; and techniques for synthesizing, processing, and characterization. This demands continuous development of advanced facilities and instrumentation.

- Structural biology, biotechnology, and the human genome. These efforts include research underlying the development of new medical treatments and commercial products, including activities exploiting such modern techniques as genetic engineering and the use of monoclonal antibodies and structural studies based on $\mathrm{x}$-ray diffraction and spectroscopy, neutron scattering, and magnetic resonance imaging. They demand continuing development of instrumentation and computation, and underpin the nation's biotechnology industry.

- High-performance computation and information management. These are efforts focused primarily in the areas of massively parallel processing and high-performance, network-mediated collaborative computing. They demand continuous development of both hardware and software as well as telecommunication technology, and are vital to the nation's economic future.

- Advanced manufacturing and industrial processes. These efforts are broadly aimed at developing and applying technology-driven techniques and scientific insights to the improvement of manufacturing methods and industrial processes. Examples include the development of steel-molding processes that eliminate costly heating and cooling cycles, the development of advanced robotics, the application of surface science research and studies or heterogeneous catalysis to crucial processes in the oil and chemical industries, and a collaboration of the textile industry with the national laboratories to explore advanced manufacturing techniques.

\section{R\&D to Secure Our Energy and Environmental Future}

- Energy supply and resource development technology. Energy supply research is aimed at improving the performance, economics, and environmental effects of technologies such as solar energy systems (e.g., photovoltaic cells, biomass combustion and conversion, and wind systems), gas fired-turbines, coal-burning systems, fission reactors, and fusion-based energy sources. Resource development technology is focused on ensuring the optimal, environmentally safe exploitation of oil, natural gas, geothermal, mineral, and water resources, as well as geosciences research to allow accurate prediction 
of natural disasters such as earthquakes and volcanic eruptions. Required activities include the development of better techniques for characterization, resource evaluation, and prediction of natural and induced processes.

- Technologies to increase the efficiency of energy use. These include fundamental research in chemical and combustion dynamics as well as development of more energy-efficient building technologies, industrial processes, and transportation systems. They encompass research on energy-efficient windows, lighting, and HVAC systems for buildings, and the development of vehicles using fuels derived from natural gas and biomass, as well as other advanced air and surface transportation systems.

- Ecological, medical, and climatological effects of energy use and mitigation of environmental contamination. These include biological and medical research on plant and animal response to pollution and climate modeling research. Solving critical problems in global warming demands advances in computation including new data management tools, parallel algorithms, speed of processing, and size of processors and storage. Environmental contamination mitigation includes research on improved characterization methods, development of predictive models and risk-assessment methodologies, development of techniques to isolate and clean up sites contaminated by toxic chemical wastes and radioactive materials (including bioremediation techniques), and R\&D on waste minimization and effective means of waste disposal.

- Energy and environmental policy. This research is aimed at optimizing U.S. policies regarding energy and environmental protection and preservation. It includes development of improved methods for analyzing policies to increase energy efficiency, assessing alternative strategies for R\&D investment and energy supply, analyzing policy responses to mitigate global climate change, evaluating environmental cleanup policy, and understanding the consequences of transferring better energy and environmental technology to the former Soviet Union and the developing world for DOE's mission.

\section{Scientific Foundations}

- The universe, matter and its interactions, and basic science as a foundation for future technology development. This effort includes research in high-energy and nuclear physics, planetary studies, astronomy, and astrophysics. Such research demands continuing development of investigative tools, including advanced accelerator/detector facilities and space technologies. Basic science studies span the scientific spectrum, including materials, chemical, geosciences, and life sciences research. Broadly based fundamental research is the ultimate source of long-term technological advancement for DOE's mission.

\section{Laboratory Capabilities and Opportunities-Addressing National Needs}

LBL's capabilities and opportunities for DOE arise from its tradition of excellence in forefront multidisciplinary research, its close working relationship with the University of California, and its ability to evolve to meet changing research priorities. LBL's long-range strategy is to focus on the 
optimal development of these strengths, while increasing its commitment to meeting national needs through expanded research partnerships with industry and the development of applied technology. LBL's core competencies were described in Section 2. Based on these competencies, the Laboratory has identified the key DOE programs and capabilities to which it can make major contributions:

- Basic Energy Sciences. The Advanced Light Source (ALS) became operational in FY 1993. It is providing the world's brightest beams of soft $x$-rays and ultraviolet light. The ALS Beamlines Initiative will help the ALS to realize its full scientific potential through the application of $x$-ray microscopy to both fundamental and applied research. Applications of the ultrahigh-brightness ultraviolet and soft $x$-ray radiation from the ALS include atomic and molecular physics; biology and medicine; chemical dynamics; materials; and industrial research technology. A Combustion Dynamics Initiative is being proposed in collaboration with Sandia National Laboratories. It combines special capabilities in physical chemistry and combustion science and offers significant opportunities to understand and improve the combustion process and prevent air pollution. Significant capabilities in atomic scale synthesis, processing, and characterization at facilities such as the National Center for Electron Microscopy and Center for X-Ray Optics offer critically important opportunities for advanced materials research.

The development of new, efficient, and clean systems for energy production, use, and transmission will be increasingly important to national research programs. This research will involve, for example, advances in the understanding and use of advanced materials and chemical processes such as combustion, high-temperature superconducting materials and technologies, alternative means of generating electricity, processes for more efficient end-use technologies, and improved methods of finding and producing fuels.

- Health and Environment. LBL's bioscience research holds promise for the understanding and prevention of both hereditary and environmentally caused disease, as well as for establishing health and environmental protection standards. LBL's Human Genome Center is a national source of expertise in automated techniques for mapping human chromosomes, sequencing selected human-gene DNA fragments, and improving data analysis and interpretation. Transgenic mouse and molecular genetics research complement efforts at the Human Genome Center by elucidating the genetic contributions in atherosclerosis and environmentally induced disease. The Advanced Light Source will give biologists access to the world's brightest source of soft $x$-rays, strengthening DOE's national position in structural biology. At the Center for Functional Imaging, advanced positron emission tomography instrumentation, radiopharmaceutical chemistry, and data analysis methodologies are joined to explore the mechanisms involved in neurological and cardiovascular disorders. Cell and molecular biologists are making important strides toward understanding the relationships between cell proliferation and cell differentiation and how these processes become deranged in cancer cells. Environmental research capabilities in pollution sensing, transport, effects, and cleanup promise to reduce costs for environmental remediation problems. 
- Energy Efficiency and Renewable Energy. LBL's capabilities extend to all the energy end-use sectors: utilities, industry, transportation, and buildings. Major contributions include substantial improvements in windows, lighting systems, insulation, thermal performance, and air quality in buildings and development of long-life, high-power rechargeable batteries for vehicles and stationary power systems. Integrated resource planning for energy utilities, geothermal energy sources, and more efficient industrial processes are among other key areas of interest.

- High-Energy and Nuclear Physics. LBL's capabilities in forefront detector systems have been demonstrated at the Tevatron, the Stanford Linear Accelerator Center, and now, with the Solenoidal Detector Collaboration at the Superconducting Super Collider Laboratory, and the STAR experiment at the Relativistic Heavy lon Collider (RHIC). LBL also continues to advance theoretical physics and develop new accelerator concepts necessary for the continuing strength of highenergy physics. The benefits will include uniquely configured, moreefficient, and more-powerful accelerators for new insights with beams of electrons, ions, and photons. LBL's nuclear physics capabilities emphasize development of new detector systems, including detectors at RHIC, that explore nucleonic and quark-gluon matter. The 88-Inch Cyclotron will provide an expanded range of light-ion and heavy-ion beams for nuclear structure studies with the Gammasphere detector now under construction. The Sudbury Neutrino Observatory will be obtaining data expected to address the solar neutrino problem and the question of neutrino oscillations. Planning for the IsoSpin Laboratory opens the possibility for nuclear physics research with an experimental program that has great promise for the coming decade.

- Fusion Energy. LBL has the special expertise and facilities needed to develop induction linear accelerator systems of a type that, suitably scaled up, would provide intense and focused heavy-ion beams capable of compressing and igniting a deuterium-tritium pellet for inertial confinement fusion. The Induction Linac Systems Experiment is a fundamental stage in development toward ultimate commercial power generation. For magnetic confinement fusion, LBL's expertise and research in test facilities for high-current neutral beams may play a key role for plasma heating systems for the International Thermonuclear Experimental Reactor.

LBL's relations with universities and industry have created new opportunities for science education and technology transfer. The San Francisco Bay Area is a center for technological innovation in the areas of biotechnology, electronics, computing industries, and large-scale engineering, to name a few. The Laboratory's collaborations with industrial partners, both regionally and nationwide, have resulted in the transfer of some of the most advanced scientific concepts, including high-temperature superconducting devices for electronics, medical accelerators, direct liquefaction of methane, and new window technology and electrochemical storage systems. The transfer of these technologies to industry represents achievements directly supporting the mission of DOE.

L.BL ties to educational institutions, including the University of California at Berkeley, other schools and colleges in the Bay Area, and the Lawrence $\mathrm{Hall}$ of Science, are resulting in a new class of partnerships that are 
achieving reforms in science education. The Bay Area Science and Technology Education Consortium and the Science and Education Academy of the Bay Area are new models for effectively using the national laboratories to advance national educational objectives.

\section{Laboratory Constraints}

Scientific and technological advances are critical if we are to achieve the energy, economic, and environmental objectives of the DOE mission. Despite the key role laboratories play in long-term economic security, national laboratory initiatives are constrained by limited budget resources. Constrained funding is a recognized factor in energy research strategic planning. The costs of state-of-the-art facilities and added operational requirements limit the opportunities for setting new research objectives. Under these conditions, it is essential that initiatives be well coordinated and managed and involve effective scientific review. Selting priorities and improving cost effectiveness will be essential to initiating new programs that are vital to the national interest.

The national laboratories are undertaking management performance improvements in many sectors of their research and support operations to meet stakeholder expectations and best management practices. These activities call for improvement in the quality of services and delivery of information at all levels, including environmental, health, and safety programs, and financial, acquisition, and personnel management systems. A laboratory must have sufficient research funding to support these raised performance expectations. Additional program growth will permit enhancement of LBL's support services while maintaining its high level of research capabilities and performance.

From the perspective of U.S. industry, national laboratories have sometimes been perceived as enclaves for government-directed research and as partners of last resort. In the past, industry has been skeptical that national laboratories can work effectively in partnerships to transfer advanced technology to industrial use. Contributing to this attitude has been the lack of a specific mission to work with industry and develop the financial resources for successful long-term research that supports critical industrial technology needs. Efforts, such as the May 1992 Critical Technologies Workshop at LBL, are under way to remove the barriers to industry-laboratory cooperation and to devote an increasing share of LBL's programs to these activities.

\section{LBL'S STRATEGY}

The strategy for achieving a vision of excellence will require $L B L$ leadership to establish compelling research priorities, build new partnerships, and sustain excellent performance by developing our people and our facilities to their fullest potential. This requires a continual commitment to quality improvement in every aspect of the Laboratory's operations and endeavors. A part of this process, as established through the new contract with the University of California, will be to establish mutually agreed upon performance criteria between the Department of Energy, University of California, and L.BL. 


\section{Strengthening Research Priorities for National Needs}

LBL's strategy for fulfilling its DOE research mission is to target those research areas that promise national benefits from the Laboratory's science and engineering expertise. The DOE mission identifies key areas of energy supply, efficiency, fundamental science, and education where the Laboratory is directing its research efforts. In concert with national reviews conducted by advisory bodies such as the Basic Energy Sciences Advisory Committee, the High Energy Physics Advisory Panel, and the Nuclear Science Advisory Committee, LBL advances research to strengthen national programs. LBL. will participate actively in DOE's strategic planning efforts to define critical new directions and establish a framework for more fully utilizing and focusing the national laboratory competencies.

For the Office of Basic Energy Sciences, the Advanced Light Source enables the Laboratory to focus its efforts more on national research priorities, including implementation of user facilities and the Combustion Dynamics Initiative. Other initiatives of a smaller scale complement these priori. ties, including upgrade of the National Center for Electron Microscopy, and advanced materials and computing research.

Research in the biosciences is becoming increasingly synergistic with energy sciences and general sciences. Facilities such as the Advanced Light Source have outstanding promise for structural biology through $x$-ray crystallography, microscopy, and spectroscopy. The Human Genome Center directly benefits from the engineering and computer science expertise originally developed for data-intensive physical sciences. The biosciences offer promise for solving significant problems in molecular medicine, environmental remediation, materials synthesis, and energy conversion. The Laboratory's priorities will continue to emphasize the potential for a national impact from these programs beyond traditional biomedical disciplines.

The Laboratory supports initiatives in the general sciences and fusion energy through established long-range planning processes. It couples its initiatives in these areas, such as the Solenoidal Detector Collaboration-SSC, the Solenoidal Tracker at RHIC. Experiment, and the Induction Linac Systems Experiment for inertial fusion energy, to the national framework for largescale scientific experiments planned for these fields.

Technology research is an emerging direction for DOE and LBL. Critical technologies research opportunities that serve the national interest are in areas such as advanced materials and processing; biotechnology; computing and information science; and energy and environmental research. The Laboratory will work with the Office of Energy Research, the Assistant Secretary for Energy Efficiency and Renewable Energy, and other program secretarial offices to support priorities for fundamental and applied research and technology transfer vital to U.S. long-term competitiveness. The Laboratory's programs in energy efficiency and storage have a strong record of effective technology transfer to industry, and support the research strategy of DOE's Energy Efficiency and Renewable Energy programs.

\section{Building Partnerships}

The Laboratory will aggressively develop research initiatives and collaborative research opportunities in concert with industrial, academic, and government partners. The success of key LBL. initiatives, including the Advanced light Source Beamlines Initiative, Induction Linac Systems 
Experiment, Combustion Dynamics Initiative, and the PEP-II B factory at SLAC will be increasingly dependent on the strengths of LBL's relationships with its collaborating partners at other national laboratories, maximizing payoffs from DOE investments without duplication.

Collaborations with industry will be essential to develop the technology research role of the multiprogram laboratories. LBL maintains a vision of leadership in becoming a model laboratory to broaden the mission of the national laboratories in response to the need for critical technologies research. The Office of Energy Research has begun support for a technology research program involving Cooperative Research and Development Agreements with LBL's industrial partners. LBL's role in contributing to national initiatives in advanced materials, biotechnology, and computing will depend on strong relationships with industrial partners. LBL's effort in establishing the California Institute for Energy Efficiency, formalized by such a cooperative agreement, provides a model for a successful collaboration among energy utilities, national laboratories, the Office of Energy Efficiency and Renewable Energy, and educational institutions.

Partnerships with other national laboratories, government agencies, and state governments will contribute to successful strategic planning for the national laboratories. LBL's research in structural biology, molecular genetics, and research medicine will greally strengthen national programs supported by both the Office of Health and Environmental Research and the National institutes of Health. LBL's alliances with other national laboratories, including Lawrence Livermore National Laboratory, Oak Ridge National Laboratory, and Stanford Linear Accelerator Center, offer promise to DOE for efficiently and effectively utilizing the expertise of the national laboratory system. The Laboratory is revising its internal organization to utilize its resources most effectively in meeting these challenges.

\section{Developing Our People and Infrastructure}

The Laboratory has a strong tradition of research quality and productivity, maintaining a commitment to the success of DOE's mission and its research programs. The Laboratory is committed to continuous improvement in workforce diversity, project performance, environment and safety management, its infrastructure, and to the use of best business practices. LBL has developed a strategy that calls for programs to improve performance based on nationally recognized criteria and is working with DOE on program implementation.

The Laboratory is accountable for program performance based on the strengths, quality, and commitment of LBL's scientific and management efforts. Its focus on innovative solutions for national research programs is based on the valued contributions of all Laboratory staff, from principal investigators and program leaders through all the technical and administrative support staff. The Laboratory's success relies on the efforts of multidisciplinary teans, where knowledgeable and capable individuals work together with common goals. The Laboratory seeks the best professional program reviews and maintains nationally recognized high standards for performance and recognition. The $D()$. review framework, from initial field lask proposal through validation and program performance reviews, is an important element of program performance measurement.

The Laboratory has established a new ( fffice of Workforce Diversity, reporting to the Laboratory Director, 10 integrate diversity into our lesearch 
culture and to provide an environment that promotes equity and excellence for all employees. This is key to LBL's success as it prepares for the $21 \mathrm{st}$ century.

Starting in 1990, LBL. undertook a cultural change that emphasizes excellence in ES\&H performance at all levels of the Laboratory. It strengthened management relationships with DOE to thoroughly support modern requirements for worker safety and health, waste minimization and management, and environmental protection.

The Laboratory is responsible for maintaining an infrastructure for effective financial and administrative performance. It is committed to best business practices in all areas of adiministration, including human resource development and training, finance and contractual relations, materials management and procurement, facilities maintenance and engineering, and other management and planning practices. The Laboratory will emphasize the application of performance measures through coordinated and nonduplicative oversight by the University and DOE. LBL is committed to working with DOE as a partner in continuous performance improvement for national research institutions. In addition, the Laboratory has centralized other functions, such as facilities management and its technology transfer programs, to streamline its operation.

\section{STRATEGIC ISSUES}

The Laboratory's ability to conduct forefront multidisciplinary research is based on its effective management of research teams, a pool of creative research scientists and engineers, and an outstanding support infrastructure. These elements form the basis for achieving a nationally significant level of scientific accomplishment while maintaining high levels of safety and environmental protection.

To maintain LBL's scientific leadership and to ensure the full development of its capabilities and resources, LBL has identified strategic management issues that it is addressing with DOE. These issues, which continue to be developed as an ongoing process within LBL's strategic planning activities, can be divided into five main categories: fulfilling our mission, implementing initiatives, modernizing our research facilities, further improving our ES\&H performance, and reducing redundant oversight and indirect costs.

\section{Fulfilling Our Mission}

Historically, the research and development missions of the DOE national laboratories have supported energy research, defense, and the physical and life sciences. As we examine our mission in today's world, we must expand our efforts to realize our R\&D capability with an eye to addressing the economic security of the nation. These changes in LBL's role can be facilitated by DOE management policies that enable the national laboratories to work more effectively with industry and with other government agencies, such as the National Institutes of Health, the National Aeronautics and Space Administration, and the Advanced Research Projects Agency. LBI. has identified the following issues that affect our ability to fulfill the Laboratory's mission: 
- Implement DOE's mission for technology research. LBL is working with DOE and other national laboratories toward a mutual goal: to develop programs that make full use of current technical capabilities to benefit the nation. To support this goal, DOE and LBL need to work together to develop programs and obtain more funding support for research initiatives that are broadly responsive to the long-term economic vitality of the nation.

- Enable industrial R\&D partnerships. The Laboratory is working with DOE to develop work-for-others conditions and Cooperative Research and Development Agreement documents and procedures that reduce obstacles to the development of R\&D partnerships and technology transfer.

- Establish further alliances and agreements with NIH, DOD, NASA, EPA, and other agencies. The Laboratory's research relationships with National institutes of Health, Department of Defense, National Aeronautics and Space Administration, and U.S. Environmental Protection Agency have strengthened national research programs in space research, health, and environment. Agreements need to be forged to reinforce these mutually constructive relationships and reduce administrative barriers.

- Fully utilize laboratory and university capabilities. LBL is unique among the multiprogram national laboratories in being located next to a large university. The Laboratory and DOE must continue working constructively to strengthen relationships with the University of California Berkeley campus as well as with other universities, and to devise an administrative framework that minimizes barriers to university and other partners seeking access to facilities. The Laboratory's unique research facilities must also be effectively utilized. For example, effective utilization of the Advanced Light Source will require additional bearnlines and the development of the second-floor offices and laboratories for non-LBL users. Utilization of these capabilities is required to gain the full benefit of the investment in these unique national facilities.

\section{Implementing Initiatives}

Limited DOE resources set constraints on the range and scope of LBL's initiatives. In this environment, scientific excellence alone is no guarantee of success; the Laboratory must also demonstrate that its initiatives contribute to national needs. The Laboratory is working with DOE, the scientific. community, state, and industry participants to establish priorities and to support initiatives that can best serve the nation under financial constraints. Examples from three key DOE program areas illustrate the Laboratory's priorities for initiatives.

- Office of Basic Energy Sciences. The Laboratory proposes additional beamlines and the completion of the second floor of the Advanced Light Source building for user offices and laboratories. The Combustion Dynamics Initiative, proposed in partnership with Sandia National Laboratories, supports DOE's Chemical Sciences Division commitment to the nation's environmental, energy supply, and fundamental science goals. The Laboratory is working with broader state and national 
scientific communities to seek additional support for this proiect to complement DOE funding. The Laboratory is also supporting improved operations funding and upgrades for DOE's forefront electron microscope capabilities, including those at LBL's National Center for Electron Microscopy.

- Office of Health and Environmental Research. LBL's Human Genome Center and the Advanced Light Source give biologists access to advanced technology needed to address fundamental life science challenges: to understand the genetic and structural basis of energyderived and environmentally derived health issues. LBL is working with the Office of Health and Environmental Research to establish a scientific and management framework that will ensure the success of these initiatives.

- Office of Fusion Energy. The proposed Induction Linac Systems Experiment project is poised to evaluate the potential for using intense and focused heavy-ion beams as drivers for inertial confinement fusion. The Laboratory is prepared to construct this test facility, as well as continuing research in other national fusion programs.

This FY 1994-1999 Institutional Plan identifies important initiatives that support other DOE programs, including the Offices of Energy Research, Energy Efficiency and Renewable Energy, and Environmental Restoration and Waste Management. To fulfill the Laboratory's mission for DOE, the timely implementation of these initiatives is essential.

\section{Modernizing Facilities}

The Laboratory will continue to give priority to modernizing and restoring facilities to sustain national programs while also achieving standards of excellence in the areas of environment, health, and safety. The Laboratory has integrated its work on the Site Development Plan with the processes of institutional planning, safety and health planning, and environmental restoration, so that a safe working environment will be provided for implementation of DOE scientific programs. Critical elements of LBL capital resource and modernization planning are implemented through:

- Multiprogram Energy Laboratories Facilities Support (MEL-FS). This program has been vital for replacing mechanical and electrical utilities, upgrading buildings, and improving segments of roadways at LBL. A key element for the program in the 1990s will be the contınued improvement of these systems and modernization of support buildings and general purpose research facilities. Many of these buildings are temporary structures, World War II vintage wooden barracks, or sheet metal structures, that do not meet the current codes or DOE/Laboratory standards. Recent reversals in the program's funding place this Laboratory infrastructure under stress.

- General Plant Projects (GPP). Small capital projects $1 \$ 100 \mathrm{~K} 10 \$ 1.2$ M) supported by GPP are essential for small program modific ations and additions, for compliance with environmental, health, and safely standards, and for upgrades of obsolete and deteriorated equipment such as transformers, switching stations, boilers, chillers, and roofs. At $\$ 3.3 \mathrm{M}$ the current annual budget for CiPP projects is inadequate to make substantial progress in meeting identified needs. In addition, 
due to rising compliance standards for capital improvement, the available funds for each project are inadequate. Therefore, raising the project ceiling from $\$ 1.2 \mathrm{M}$ to $\$ 2 \mathrm{M}$ would also assist the Laboratory's rehabilitation plan. LBL also recommends that the Office of Energy Research and the Office of Laboratory Management coordinate GPP plans to ensure a coherent framework for GPP improvements for the multiprogram laboratories.

- General Purpose Equipment (GPE). The Laboratory uses GPE funds to replace its essential support equipment. This equipment includes environmental, safety, and health equipment; essential physical plant equipment for maintenance and repairs; mechanical and electrical engineering shop equipment; transportation vehicles, including shuttle vehicles; data processing and telecommunications equipment; and other equipment used by support divisions. In FY 1981 support for GPE was $\$ 1.3 \mathrm{M}$ per year, a level that allowed essential services to continue but did not reverse obsolescence. During the next few years funding slid to $\$ 800 \mathrm{~K}$. In FY 1993, GPE funds are $\$ 1.8 \mathrm{M}$, but, compared with 1981, the purchasing power is equivalent to about $\$ 700 \mathrm{~K}$, or one-half the 1981 funding. This level of need has resulted in equipment being used beyond its normal lifetime, sustained high maintenance costs, and substandard equipment performance.

- Bevalac decommissioning. LBL has developed a decommissioning plan for support and program facilities. The Bevalac accelerators were shutdown in FY 1993. Plans are for Energy Research support for the stand-down and secure phase into FY 1994, while subsequent plans for the decommissioning of the accelerator are being developed as discussed further in Section 9. It is important that funds to sustain the standard and secure operations be provided in FY 1995 and until acceptance by Environmental Restoration and Waste Management. The value of the Bevalac site, buildings, and its location within an urban area make this project a very high priority for the Laboratory and the University.

\section{Improving ES\&H Performance}

It is the policy of the Laboratory to integrate its performance in the areas of environment, safety, and health (ES\&H) into the planning and conduct of all of its operations to protect the health of employees, the public, and the environment. The Laboratory has developed a comprehensive Corrective Action Plan, Five-Year Environment, Safety and Health Plans, and Environmental Restoration and Waste Management Plans that integrate ES\&H requirements into all Lab activities. All new initiatives include specific identification of ES\&H needs, and their Conceptual Design Reports and other supporting materials indicate ES\&H requirements for program planning. Laboratory leadership and staff continue to make every effort to maintain the momentum and support the culture change mandated by LBL's Self-Assessment Program and the Tiger Team Assessment Corrective Action Plan. The following critical issues continue to be addressed:

- Resources for required environment, safety, and health plans. Management arrangements and interactions with DOE are being strengthened, and commitments must be obtained from DOE to ensure that the ES\&H momentum can be maintained. Funding through the Office of Energy Research (ER) has been insufficient to fully develop and 
implement LBL's ES\&H plans and programs. The Laboratory's FY 1993 programs have been conducted with 762 support FTEs, serving 1812 direct FTEs. To fulfill support demands at the projected levels of expectation for ES\&H and administrative management, additional indirect FTEs are required. Within the existing burden levels this will require programmatic growth to approximately 2000 direct FTEs. This overall growth to a critical size will enable the Laboratory to meet its Corrective Action Plans for ES\&H and administrative compliance while maintaining program objectives.

- ES\&H facilities for essential programs. As indicated by the Tiger Team, many of LBL's ES\&H facilities are inadequate and in poor condition. A Safety and Support Research Facility and an Environmental Monitoring and Industrial Hygiene Building are necessary to correct deficiencies identified by the 1991 Tiger Team Assessment, implement the Corrective Action Plan, and fulfill the personnel and program obligations in Environmental Restoration and Waste Management and Environment, Safety and Health five-year plans. The facilities will allow the Laboratory to meet accepted standards for LBL's environmental, health, and safety programs, including providing adequate monitoring and sample processing laboratories, adequate emergency command and response facilities, and sufficient space for on-site offices for industrial hygiene, environmental, and other essential ES\&H staff. Construction has begun on a New Hazardous Waste Handling Facility to upgrade, consolidate, and relocate existing waste handling operations. Construction is scheduled for completion in 1996.

- Clarifying ES\&H roles, responsibilities, and funding. LBL is working with DOE to use its resources more effectively in the ES\&H area. A major area for refining ES\&H responsibilities would be in identifying, prioritizing, and funding for preventative measures to preclude damaging and expensive environmental conditions. Funding is not available, for example, for waste minimization activities. On the other hand, funds to remediate soil and groundwater are available. DOE funds could be used more effectively by better balancing funding between preventative measures and after-the-fact measures, and by effectively integrating the responsibilities and resources of the Office of Energy Research and Office of Laboratory Management with those of the Environmental Restoration and Waste Management organization.

\section{Reducing Redundant Oversight}

DOE, LBL, and UC need to work together to eliminate redundancy in the review process, such as minimizing redundant paperwork and lengthy review cycles in the existing procedures for compliance with presently required for the National Environmental Protection Act (NEPA). A revised system for complying with DOE Orders, such as the Self-Assessment Program, hold promise for improving the efficiency of laboratory operations.

- Paperwork reduction. The existing framework for Cooperative Research and Development Agreements must be streamlined and made more timely. In addition, paperwork required for NEPA compliance should be reduced. Both of these actions would facilitate effective decision making and encourage industrial partnerships. 
- Excessive reviews. LBL program and support activities are subject to more than 300 reviews annually. These costly reviews require considerable time and effort by both the Laboratory and DOE. By emphasizing biennial and triennial reviews DOE could streamline the review process, increase productivity and reduce costs. The current contract between the University of California Regents and the Department of Energy calls for the elimination of redundant oversight, and every effort should be made to address this requirement for the benefit of the Laboratory and DOE.

$D O E, L B L$, and UC need to work to better understand the drivers of indirect costs. Opportunities for consolidating redundant and/or non-valueadding activities should be identified. In addition, rigorous implementation of the new DOE Directives Process is needed to ensure the cost impact and usefulness of proposed orders are identified before they are issued as final requirements. Coordination among DOE Offices of Principal Interests would be helpful to eliminate conflicting and overlapping requirements. 


\title{
4 initiatives
}

\begin{abstract}
Build on our educational and technical resources to gain a competitive advantage for addressing problems of national significance and advancing the mission of the DOE.... Make $L B L$ the location of choice for facilities and programs.
\end{abstract}

LBL Vision 2000

The Laboratory's initiatives have goals appropriate to a DOE national laboratory and are capable of significant new scientific and technological achievement. Expanded research program activity of a smaller scale is summarized in Section 5, Scientific and Technical Programs. Environment, safety, and health initiatives that support the Laboratory's services and support programs are described in Section 6, and human resources and information resources initiatives are described in Sections 8 and 10, respectively. The proposed initiatives listed below encompass the five-year planning period and span DOE's research program areas appropriate to this multiprogram national laboratory. Inclusion of the initiatives does not imply that they have been adopted by DOE or that they will be funded at the levels requested. Estimates of the approximate resource requirements for these initiatives include the incremental operating costs (with equipment) and construction costs over the period of the plan. Each initiative incorporates environmental, safety, and health compliance requirements and cost, including NEPA and CEQA review requirements.

\section{Basic Energy Sciences}

- Combustion Dynamics Initiative

- Advanced Light Source Beamlines Initiative

- Atomic Scale Synthesis of Advanced Materials

- NCEM Upgrade

- High Performance Computing and Communications 


\section{High-Energy and Nuclear Physics}

- PEP-II, An Asymmetric B Factory

- SSC Solenoidal Detector Collaboration

- Relativistic Heavy-lon Collider Program

- IsoSpin Laboratory

\section{Health and Environmental Research}

- Human Genome Laboratory

- Structural Biology Initiative

- Clobal Change Research Program

- National Biomedical Tracer Facility

\section{Energy Efficiency and Renewable Energy}

- Advanced Energy Design and Operation Technologies

\section{Domestic and International Energy Policy}

- Assisting Deployment of Energy Practices and Technologies

\section{Fusion Energy}

- Induction Linac Systems Experiments

- Neutral Beam Test Facility for ITER

\section{Environmental Restoration and Waste Management}

- Environmental Restoration Research and Development

\section{Work for Others}

- Advanced Lithography Initiative

- Magnetic Materials Microscope

\section{BASIC ENERGY SCIENCES}

\section{Combustion Dynamics Initiative}

In support of DOE's national role in combustion research and chemical science, LBL and the Sandia National Laboratories (SNL) have proposed a Combustion Dynamics Initiative. This initiative advances DOE's energy sciences mission by providing the knowledge and technology base needed to help solve the nation's combustion-related problems of the 21 st century. DOE's Basic Energy Sciences Advisory Committee (BESAC) reviewed the initiative in depth in 1991 and gave its strong endorsement to facility constiuction, saying, "The Chemical Dynamics Research Laboratory (CDRL) at Berkeley, which along with the Combustion Research Facility, Phase II, comprise the facilities portion of the Combustion Dynamics Initiative should be initiated. The CDRL will enable research that is also at the heart of the 


\section{U.S. ENERGY CONSUMPTION}

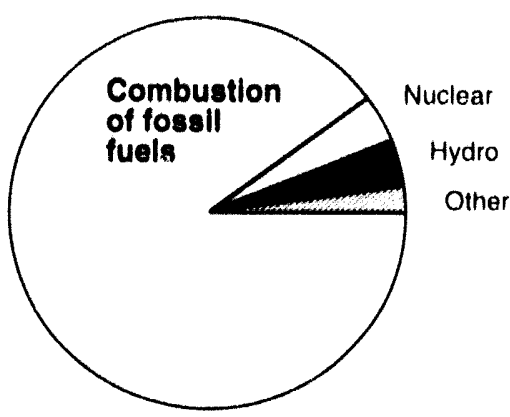

L'S. dependence on fossil tuels will not change appreciably in the foreseeable future. It is this dependence that gives a compelling reason for advanced research into the mechanism of fossil fuel combustion.
OBES and DOF mission in support of energy development and efficiency, while making use of the special capabilities of the ALS." The CDRL also contributes to the conservation energy programs in DOE, including efficient use of methane and related gaseous and liquid fuels.

The CDRL will provide unparalleled experimental resources for national users to enable new investigations of fundamental and applied combustion processes. At LBL, new lasers, experimental stations, and dedicated chemical physics beamlines from the Advanced Light Source (ALS) will be made available for dynamic, spectroscopic, and structural studies of many types of highly reactive molecules, radicals, clusters, and unusual transient species. A rigorous molecular-level understanding of combustion reactions, the structure and dynamics of highly excited molecular species and reactive intermediates, and molecular energy-flow processes can provide basic new knowledge that underlies scientific and technological leadership in internationally competitive energy-related industries and alternative fuels. Application of this basic chemical knowledge will be accelerated by the partnership with SNL through complementary experimental resources for applied research and linkages to combustion researchers in industry and universities.

The CDRL's purpose is to achieve this new understanding by an intense experimental and theoretical effort, developing and applying infrared radiation sources, and ultrahigh resolution and picosecond lasers in the VUV region, as well as molecular-beam apparatus for these investigations. The proposed facilities include:

- A tunable high-power infrared solid state laser optimized in the 3-to $10-\mu \mathrm{m}$ wavelength region for chemical reaction research;

- Advanced lasers, molecular-beam machines, fully equipped experimental stations, ALS chemical physics beamlines, and computer-based modeling and control systems; and

- A laboratory facility to support and provide utilities, safety systems, and necessary space to conduct studies.

The solid state laser will, for example, allow scientists to excite the internal modes of molecular species in a way that simulates the combustion environment and. with high intensities and uniquely broad tunability, will allow the unprecedented capability of selective multiphoton excitation. The research facilities would be assembled by combining the various photon sources with molecular-beam and ultrahigh-vacuum surface apparatus. The high-intensity photons from the ALS VUV undulator and the successful development of a high-resolution VUV laser at LBL will expand the potential scope of experiments substantially, making it possible to monitor many spectroscopic and reactive-scattering processes that were not possible to observe in the past. The facility will allow, for the first time ever, the integrated and simultaneous use of high-power IR laser and ALS beamlines for pump-probe experiments with crossed molecular beams.

Possible new pump-probe experiments include the high-resolution infrared spectroscopy of intermediates in molecular beams and the probing of reaction-product state distributions. The short pulse durations and synchronization of the VUV photon sources will make possible fast-timing experiments, such as the measurements of intramolecular relaxation and rearrangement rates, and unprecedented high-resolution photoelectron spectroscopy experiments. The facility's scope also allows for the flexible management and arrangement of experimental apparatus, for safe transfer of 
The Chermical Dynamics Research $L a b o r$ atery at $L B L$ and the Combustion Research Facility II at Sandia National Laboratory will be important new resourcess for the understanding of combustion chemistry and fluid dymamics. Working in partnership with industry and universities, the Combustion Dynamics Initiative will play a signiticant role in technology development and transfer to industry as well as supporting the $D()$. mission in energy development and efficiency. chemicals and gases, and for modular instrumentation and computer interface systems.

Research results from the CDRL will be incorporated into powerful predictive computer codes under development in the Advanced Combustion Modeling Environment Initiative. These computer codes will enable a broad range of manufacturers to design clean, efficient combustion systems. Combustion modeling is discussed further in the section on High Performance Computing and Communications.

The CDRL will host visiting scientists, and its facilities will be made available to all qualified collaborating investigators. A Program Review Panel, reporting to the highest levels of management, will recommend allocations of resources and review all proposals for use of the experimental stations. LBL's outstanding graduate student and science education programs will contribute to full utilization of the facility in support of national science education goals. The majority of research at the CDRL will be conducted by graduate students and postdocs from participating universities. A Steering Committee of predominantly external advisors and strong industry representation will be appointed to provide advice on policy issues and to ensure maximal scientific and technical productivity of the facilities to achieve national scientific purposes. Collaborations of external users and in-house research personnel will be supported by a dedicated scientific and technical staff. A Fellows Program will attract outstanding scientists to the CDRL. The planning of this initiative has included rigorous safety, health, and environmental considerations to ensure that the project will be conducted in full compliance with DOE Orders, NEPA and CEQA, and other federal, state, and local regulations. It is anticipated that an Environmental Assessment will be prepared under NEPA and an Initial Study will be prepared under CEQA.

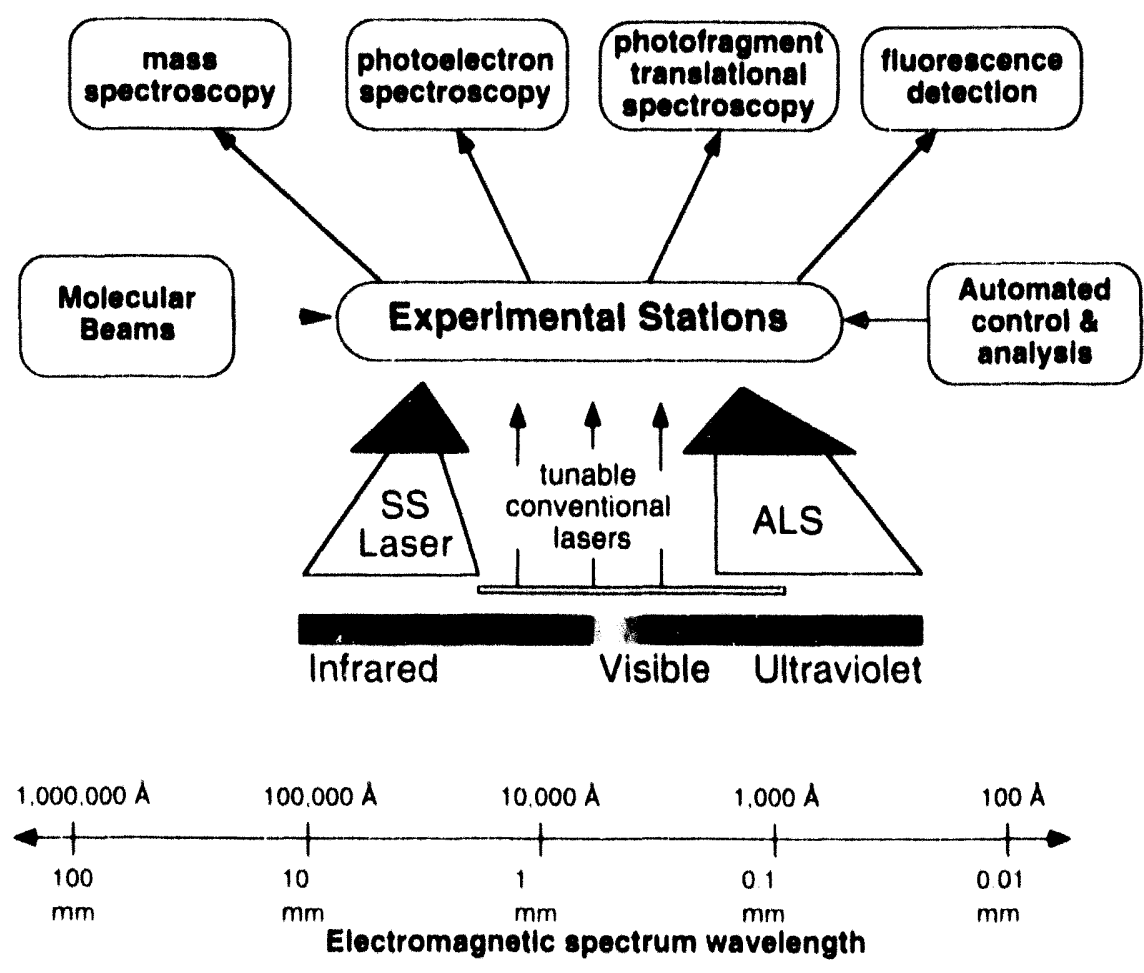


Combustion Dynamics Initiative Resource Requirements (SM)*

\begin{tabular}{lccccccc}
\hline Category & 1994 & 1995 & 1996 & 1997 & 1998 & 1999 & Total \\
\hline Operating & 0.0 & 0.0 & 1.0 & 2.0 & 2.0 & 2.0 & $7.0^{b}$ \\
Construction & 0.0 & 0.0 & 6.9 & 16.2 & 15.2 & 10.5 & 48.8 \\
\hline
\end{tabular}

"Estimates of actual-year Budget Authority for construction and relded project costs for the Chemical Dynamics Research Laboratory (B\&R cokle KC).

boes not include \$0.3M R\&D conducted in FY 1992.

\section{Advanced Light Source Beamlines Initiative}

To realize the full scientific potential of the Advanced Light Source (ALS) for both fundamental and applied research, LBL is proposing an initiative for experimental facilities needed to address new forefront scientific and technological opportunities identified by the nation's research communities. Applications of the ultrahigh-brightness ultraviolet and soft $x$-ray radiation from the ALS cover diverse areas, including atornic and molecular physics; biology and medicine; chemical dynamics; materials, interface, and surface science; and industrial research and technology:

- Several of the most exciting applications of the ALS lie in the field of high-resolution $x$-ray microscopy where enhanced spatial resolution is a signific ant consequence of the high brightness of the ALS. The impacts of $x$-ray microscopy are expected to be broad because of the decreasing size of the physical and biological systems to be analyzed.

- Use of novel two-photon techniques will make it possible to do realtime measurements of dynamical phenomena in materials.

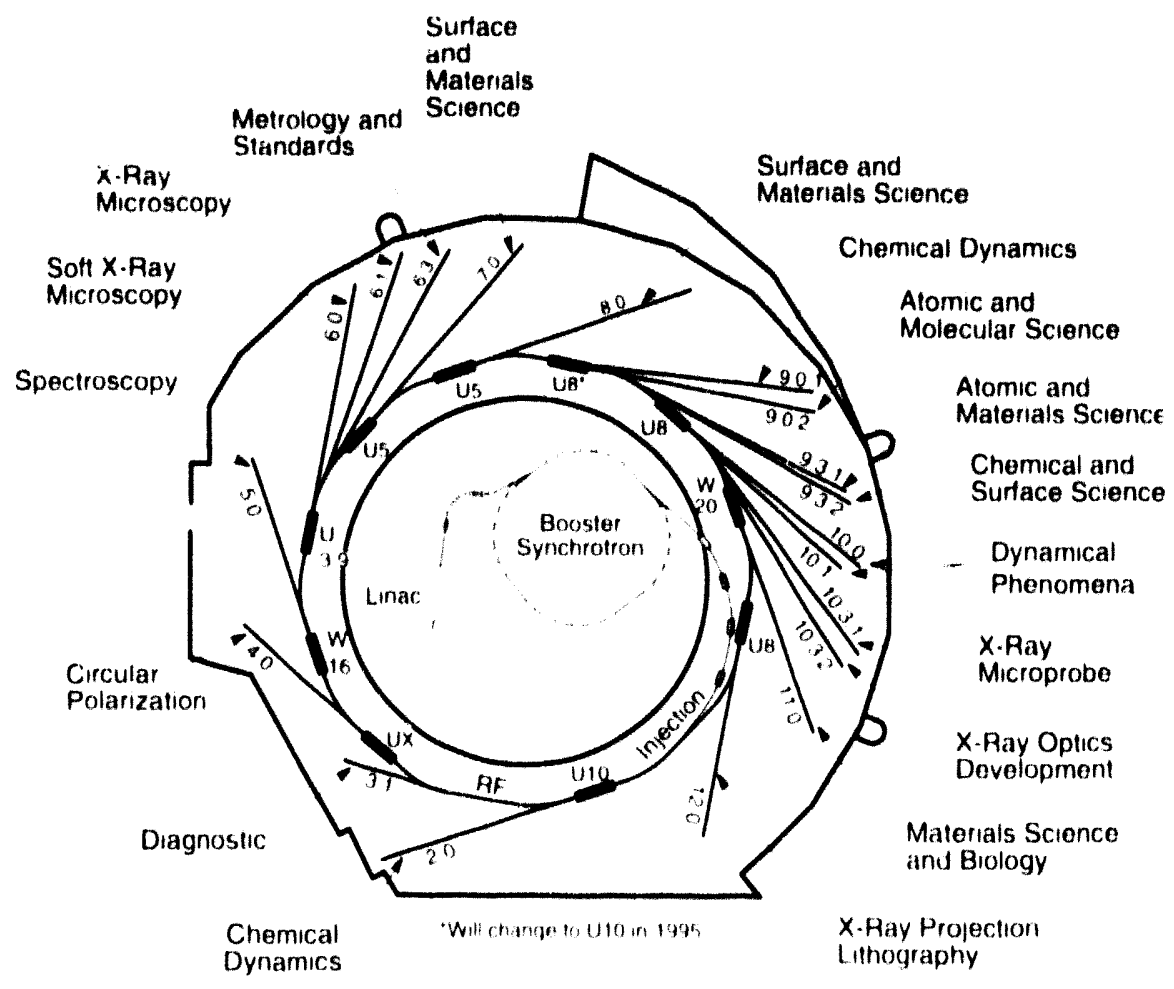

A diagram of the ALS llowe shows the beamlines planned for construction through 1998. The numbering scheme for bedmlines is related to the storage-ring sector where the'y begin. The ALS Beamlines Initiative will add insertion-device bedmlimes as well as user facilities such as support laboratories and offices in the ALS building. 
- The pulsed time structure of the ALS is crucial to the area of chemical dynamics, which encompasses all phenomena in which molecules undergo energetic or rhemic al transformations, both in the gas phase and on solid surfaces.

- The availability of broad-spectrum x-ray radiation is essential for detailed spectroscopic studies of solid-state materials and gas-phase atomic and molecular systems.

- In all fields, the extra degree of freedom associaled with circular polarization lends a precision and sperificity to photon-based techniques that is otherwise lacking. The use of circularly polarized synchrotron radiation is now at the culting edge of research on properlies, such as magnetism, that are dependent on electron spin.

Full utilization of the ALS to exploit emerging needs of users from industry, academia, and government laboratories requires insertion devices (undulators and wigglers) and beamlines to carry the light from insertion devices and bend magnets to the experimental end stations, together with completion of the $1877 \mathrm{gsm}(20,200 \mathrm{gsf})$ of office, light laboratory, and support space for users in the unfinished second floor of the ALS building.

An LBL technolan and an engineer prepare a monochromoter housing tor mas hiring on a giant computer-controlled mill. The monochromoler will be ased in one of the experimental areas at the Advanced light Source.

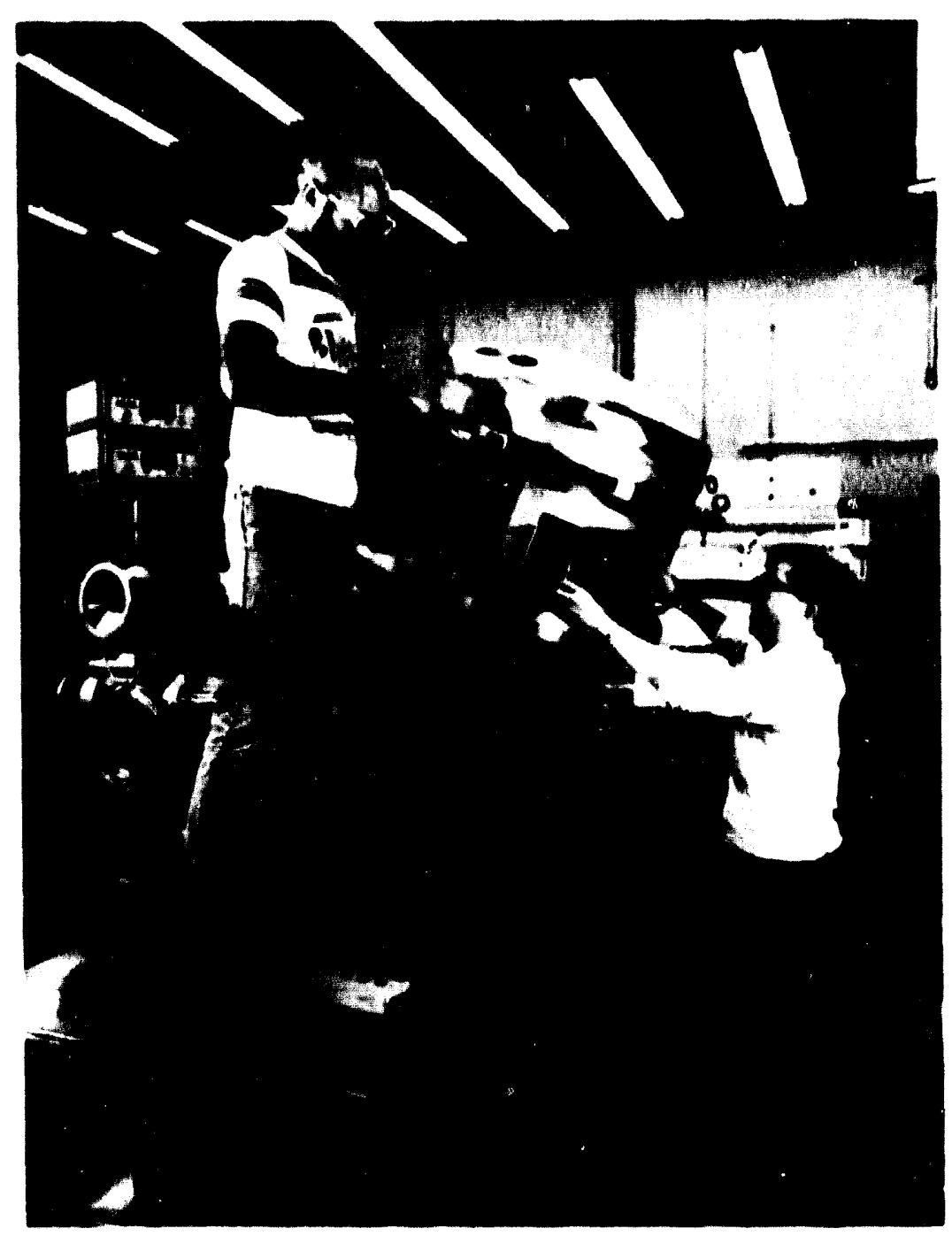


Project costs include all safely systems necessary for full compliance with applicable regulations, orders, and ALS design specific ations. In compli. ance with NEPA an Environmental Assessment was prepared for the ALS and a finding of No Significant Impacl was issued in 1988. In compllance with CEQA, an Initial Study report was prepared and a Negative Declaration adopted in 1987.

Advanced Light Source Beamlines Initiative Resource Requirements (SM)"

\begin{tabular}{lccccccc}
\hline Category & $\begin{array}{c}\text { Prior } \\
\text { Years }\end{array}$ & 1994 & 1995 & 1996 & 1997 & 1998 & Total \\
\hline Operating & 0.1 & 0.0 & 3.0 & 2.0 & 3.0 & 0.0 & 8.1 \\
Construction & 0.0 & 0.0 & 9.3 & 21.7 & 24.0 & 0.0 & 55.0 \\
\hline
\end{tabular}

Apeliminary estimate of actual-year LBL. Budget Authority (BRR coxde KC).

"For production of conceptual design repon and environmental assessment.

\section{Atomic Scale Synthesis of Advanced Materials}

In support of DOE's materials science research programs, LBL is propos. ing the establishment of a new program to develop techniques for the synthesis, processing, and characterization of materials at the atomic scale. Alomic scale synthesis will enable highly efficient, finely controlled processing and fabrication for the development of a new realm of materials. In addition, atomic scale processing can enhance the properties and synthesis techniques for existing thin films and their use in conjunction with bulk materials. The effort will strengthen DOE's mission in basic research that supports arlvanced energy technology, enabling more effective development of energy sources and more efficient processing technology. Alomic scale

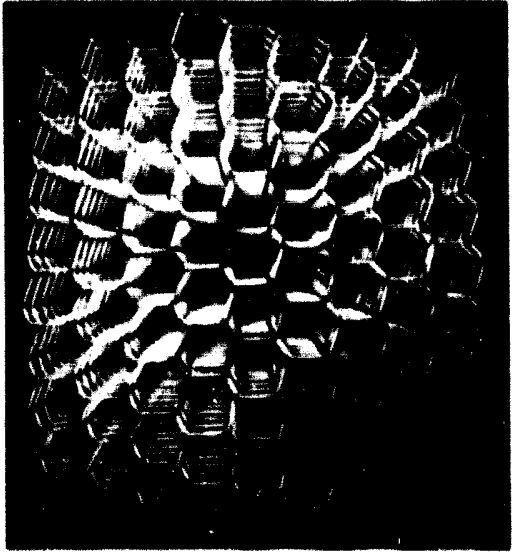

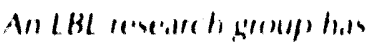

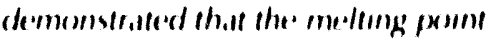
of sememeonductor nomocrystils is dependernt on the size. al the cluster. through the werecolelectrom

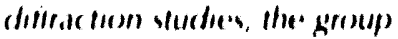
demomstralted a doubleng of the. melteng termpersallure of the direct.

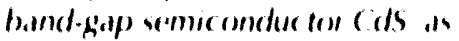

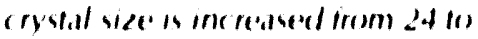
6.4 . control will enable a new generation of materials technology to improve energy efficiency, environmental research, and economic compelitiveness as exemplified below:

- New superconductors and semiconductor structures lenergy storage. photovoltaics, sensors, computing, and automation)

- High-energy-product magnets and magnetic films (motors and memories)

- Control of friction (energy dissipation)

- Hard and/or soft coatings (wearing)

- Stronger and lighter structural materials (transport)

- Materials with better heat resistance (energy dissipation)

- Catalysis (coal gasification and liquefaction; envirommental cleanup).

The program will extend beyond the current generation of thin films, interfaces, and bulk materials resedrch. These next-generation studies can enable atomic ally hilored materials optimized for energy storage, energy transfer, and optical, mechanical, electronic, surface llubricalive, adhesive, hardness), and magnetic properties. (optimized characteristics can be achieved through manipulation and deposition of atoms, clusters, and films using novel inorganic and organic synthesis, molecular and ion manipula- 


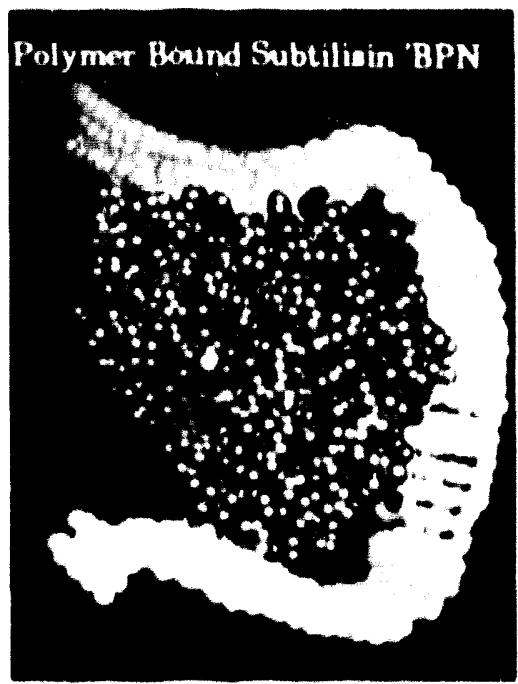

New miternals such as the profured polymer shown bound to sublilisin BlPN, have been discovered thalt stabilize proterns. enstbling them to tefalin activity al televited remperaftures and in organic solvents. This discosery allews an increased understanding an the

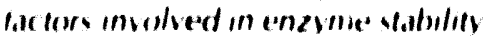

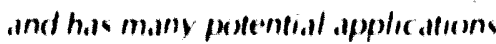
in the indualrial wae of enzymes and in diggnostes. The figure is a computer moxtel of cialsohydrille. hosed polymer emelepung the encume subelisum. tion, and other technicues, resulting in unique "zero-dimensional" structures or quantum dots (points at conventional scales), molecular wires, tubes of one dimension (length), and iwo-dimensional films of atomic or molecular thickness. These unique building blocks can be assembled into novel structures with advaliced performance (apabilities, such as near-zero noise, unprecedented spectral resolution, and temporal response that goes beyond conventional materials into the femtosecond domain.

The program includes the development of low-dimensional building blocks, uilizing organic biological methods and inorganic synthesis. A wide range of techniques will be employed, including solution chemistry for the preparation of zero-dimensional crystallites of cluster compounds, the preparation of polymeric molecular wires sheathed in nonconducting molecules, and the use of organic linkers to allach dots and wires to surfaces. Powerful characterization methoxls are available at LBL, including the Atomic Resolution Microscope (see below), advanced laser spectroscopy, and the AL.S. The initiative complements the ALS, which will be invaluable for studies of structure 10.g., with glancing-incidence $x$-ray scaltering) and other properties. Structural charac lerization will also be performed by LBL's scanning lunneling micro. scopes and altomic force mic rosc opes to characterize hardness, athesion, and other properties. The research will be conducted under rigorous safety, health, and environmental controls and reviews to ensure compliance with DOE Orders and (OSHA regulations. (ollaborations with indusiry will be encouraged, and an industrial visitors program will be established. As research proposals under this program are developed, they will be reviewed for NEPA and CEC QA compliance requirements.

Atomic Scale Synthesio of Advanced Materials Resource Requirements (SM)d

\begin{tabular}{lccccccc}
\hline Category & 1994 & 1995 & 1996 & 1997 & 1998 & 1999 & Tolal \\
\hline Operating & 5.2 & 5.2 & 5.2 & 5.2 & 5.2 & 5.2 & 11.2 \\
fquipment & 3.0 & 2.0 & 2.0 & 2.0 & 2.0 & 2.0 & 13.0
\end{tabular}

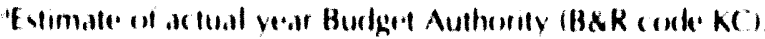

\section{NCEM Upgrade}

In support of the national research program in materials science, IBL is proposing an upgrade of its National Center for Electron Microscopy (NC EM). This national user fac ility has kept 1$)(x$ programs at the leading edge of transmission electron mic roscopy during the decade of the 1980s, particularly in high resolution, where the Alomic Resolution Microscope has been in the vanguard of the world effort. The NCEM Upgrade is for a ()neAngstrom Mic roscope with a spaltal resolution $50 \%$ grealter than current instruments. This initiative also complements a propossal for a Magnedic Materials Microscepe for materials charac ferization in a field free leons enviromment. This hatter fac ility is being proposed as a separalle Worh-for

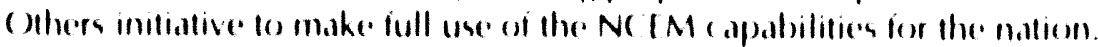

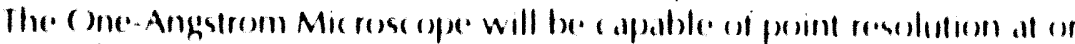

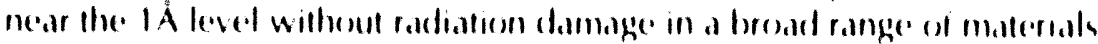

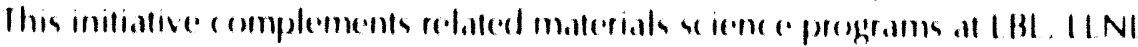
and SNI and supports a range of prestrams funded by the (oflice en Basis

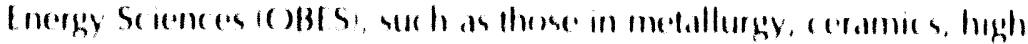

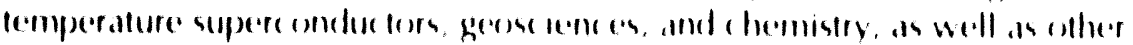




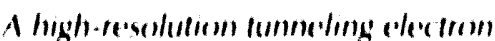

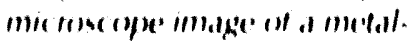

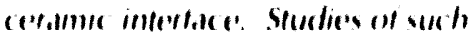

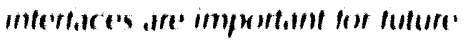

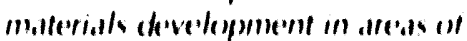

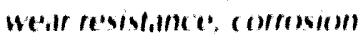
protection, andelectronics. Courent lechmolengy is limited to resolution on 1.6. A. insutficient for observing me'bll linteices in most oricentations.

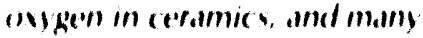
defects. The propused NCEAic he. Angstrom Mlic roscolese will allow

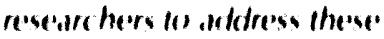
fund.mementil melterials problems.

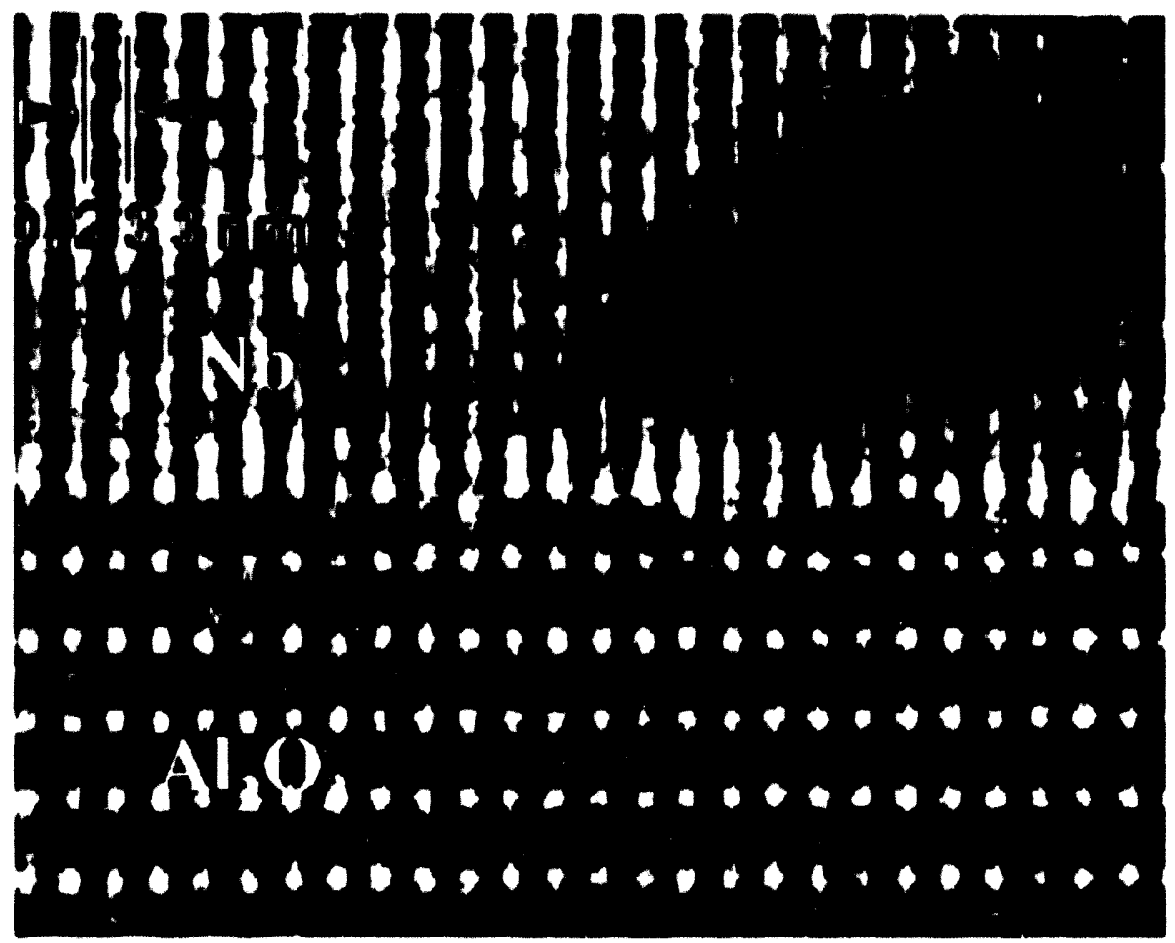

Oifice of Energy Research programs, inc luding the life sc iencess. It enables

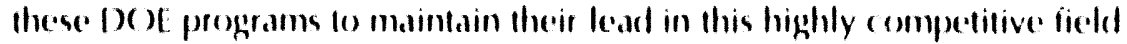
through the next dec ade.

In addition, the upgrade comprises state-of the ant support instrumentation to strengthen and complement the exosting fac ility. This inc leuder new in-situ Capabilities for direc lly relating clec tric al properties to micresstruc fural events; an energy - filtered analytic al micrescope, a Z-contrast instrument for high resolution mass contrast inngging and other suppoont instrumentalion. New

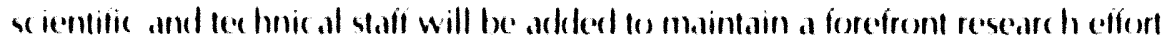
and (1) make the center's apabilitien readily available to a wide range of seientists from a ademic, industrial and government laboratories.

NC IN'sexisting comprehensive computational capability will be expanded and integrated with new mic roscopes to sutemste instrument control. alignenent and operation. The develepenent of the computer-

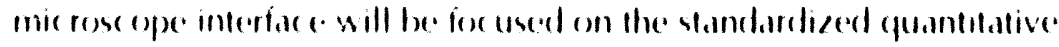

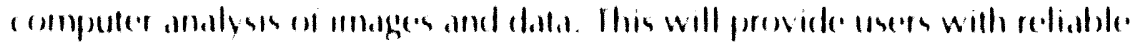

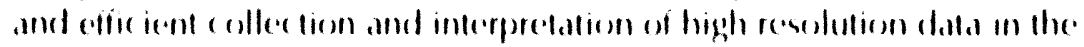

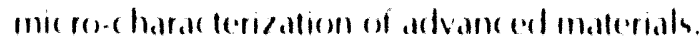

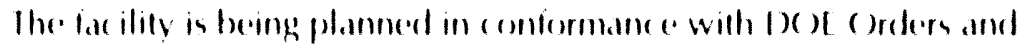

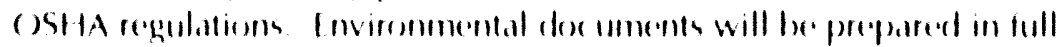

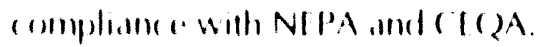

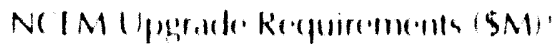

\begin{tabular}{|c|c|c|c|c|c|c|c|}
\hline ( allogent & 199.4 & $190 \%$ & $1 \% 41$ & $140 \%$ & 14) & 19109 & $|n, 1,1|$ \\
\hline ( ) & 3.11 & 11 & 1.1 & 1.2 & $\therefore 1$ & 4.4 & 1119 \\
\hline ( Imstrut ticm) & 0.11 & 1111 & $(1,1)$ & $(1,1)$ & $11 . !$ & 11.4 & 11 \\
\hline
\end{tabular}




\section{High Performance Computing and Communications}

The primary goal of the High Performance Computing and Communications Initiative is to enhannce national research and domestic economic devel. opment lhrough high-speed, advanced computation, and data communic ations capabilities. This initiative responds to the need for advanced information highways that meet Americ a's information needs. It addresses the critic al relationship be:ween successful federal scientific research and enabling high performance compuling and communications. The initiative stresses the importance of the U.S. computer industry to the national economy. Additional Information Resources Manlagement Initiatives are included in Section 10.

The DOE and LBL programs place emphasis on major scientific initiatives, including the development of software tools, new computational capabilities, and gigabit data transmission network applications. As part of the DOE program, LBL has an R\&D strategy based on developing an integrated, distributed computing environment, including advanced workstation capability, computer servers, database servers, and a high-speed network integrated through a software bus environment.

LBL is developing key technologies as a part of its high performance computing research program, including high-speed networking, advances in the software bus, developing scientific databases, and imaging and visualization tools for multimedia capability. These tools are being applied to Cirand challenges, including combustion modeling research, resolutionlimited imaging (in the areas of medicine, structural biology, geology, and AlS imaging systems), global (limate modeling, human genome research, and development of the next generation of detectors for nuclear physics and the Superconducting Super Collider (SSC).

An important part of the effort is focused on the problem of building high-speed distributed computing applic ations over heterogeneous networks. The fundamental limit is the overhead in inter-process communication mechanisms, network protocols, and routing algorithms that limit performance to a small fraction of the theoretical bandwidth. The research program utilizes existing lesthed networks to improve performance limits for problems of interest to the scientific and engineering community. In order to determine these performance limits, the Information and Computing Sciences Division is currently working with the XUNet collaboration, using 1345 megabitsecond) networks to model and measure network characteristics and develop enhancements to existing protocols and inter-process communic ation mechanisms. The program will also utilize the Bay Area Cigabit Network to improve network performance for distributed computing and multimedia applications.

An area of special emphasis will be new capabilities in combustion modeling. This inc ludes calls for the nexl generation of combustion devices with minimal emissions and maximal fuel eiiiciency. The common theme of the modeling efforts is to understand the combustion chemistry and the interaction of chemistry with fluid flow. This understanding will allow scientists to fillow the develepment of pollutants in combustion devices and minimize them. while at the same time maximizing fuel effic iency. Both stationary and mobile combustors will be studied, inc luding both gas and diesenl engines, industrial burners and furnaces, and gas turbines. The Advanced Combustion Modeling Environment is an initiative that addresses the relation ship among combustion problems and combustion modeling approaches that can contribwte to solutions for engine/ ombustor denign needs. 
High Performance computing and communications Resoures Reyuirements $(\$ M)^{4}$

\begin{tabular}{llllllll}
\hline Category & 1994 & 1995 & 1996 & 1997 & 1998 & 1999 & I0tal \\
\hline Operating & 4.8 & 5.0 & 5.5 & 6.0 & 6.0 & 6.0 & 11.3 \\
Construction & 0.0 & 0.0 & 0.0 & 0.0 & 0.0 & 00 & 0.0 \\
\hline
\end{tabular}

"Preliminary estimate of I.BL actual year Budget Autherity (BSR code KC).

\section{HIGH-ENERGY AND NUCLEAR PHYSICS}

\section{PEP-II, An Asymmetric B Factory at PEP}

The study of B-meson decays will be one of the key elements of the worldwide high-energy physics program for many years to come. These studies are limited today by the relatively low rate of events produced at $\mathrm{e}^{+} \mathrm{e}^{-}$storage rings such as the Comell clectron Storage Ring. To study the most interesting processes within the Standard Model, hoth rare decays and. even more important, the phenomenon of charge conjugation-parity (CP) violation, an effective increase in the event rate of at least a factor of 100 is required. In PEP-II, this will be accomplished by increasing the luminosily by a factor of 10 and by simultaneous'y increasing the event sensitivity through the use of asymmetric collisions (equivalent to another factor of 10 in luminosityl, as described below.

The concept of using asymmetric collisions of storage-ring beams with a center-of-mass energy at the Upsilon (4S) resonance was originally suggested at LBL. The Upsilon (4S) decays into Iwo B mesons nearly at rest in the center of mass. Since the center of mass would be moving in the laboratory frame bec ause of the different beam energies of the two ringst,

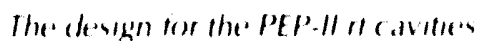
has beens studied externavely. Teats on the low posver prostentye shown here have led un to begin the deseghn ot a hegh-pewere protolvese. The.

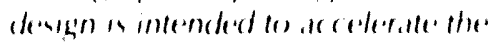
bestm with the tumblamental mode.

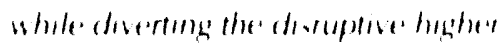

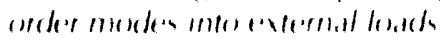

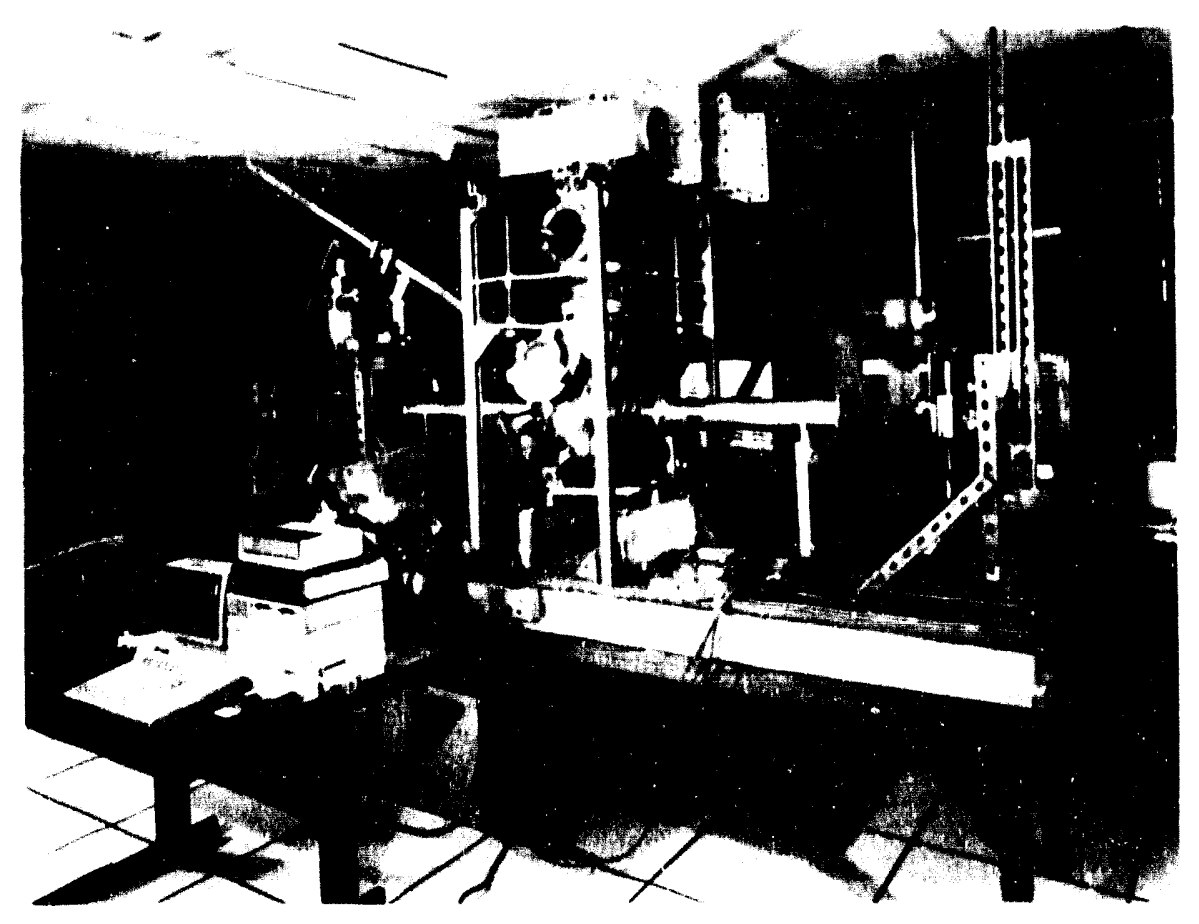


the two $B$ mesons move along the direction of the higher momentum beam, and their decays are separated in space (or equivalently, time). This separation permits the reconstruction of the individual $B$ mesons and the study of the time evolution of their decays. As noted, the use of asymmetric collisions is equivalent to an additional factor of approximately 10 in luminosity for the study of the most interesting channels for $\mathrm{CP}$ violation.

LBL, in collaboration with the Stanford Linear Accelerator Center (SLAC) and LLNL, has evaluated the use of the Positron-Electron Project (PEP) storage ring in conjunction with a new low-energy storage ring. A B-factory based on PEP (which serves as the high-energy, 9-GeV, ring), with the addition of a new low-energy (3.1-GeV) ring, is attractive both scientifically and fiscally. A conceptual design, prepared in collaboration with SLAC and LLNL, has shown that such an asymmetric $B$-factory scenario is entirely feasible with state-of-the-art technology.

The construction of the PEP-based B-factory (PEP-II) would be carried out as a collaboration among SLAC, LBL, and LLNL. LBL would play a lead role in major aspects of the accelerator construction project. Studies are also presently under way to design a new detector to exploit fully the opportunities made possible with PEP-II. The detector construction and operation would be carried out in collaboration with universities and other national laboratories. LBL physicists would participate in the detector design and construction, and would play an important role in the excellent scientific program to be carried out at the facility. All environment, safety, and health requirements are included in this project.

PEP-II Asymmetric B Factory Resource Requirements (\$M) a

\begin{tabular}{lrrrrrrrr}
\hline Category & 1994 & 1995 & 1996 & 1997 & 1998 & 1999 & Total \\
\hline Operating & 1.6 & 1.0 & 0.0 & 1.6 & 6.9 & - & $31.5^{b}$ \\
Construction & 36.0 & 44.0 & 52.0 & 45.0 & - & - & 177.0 \\
\hline
\end{tabular}

"Includes SLAC/LBL/LLNL costs. Preliminary estimate of actual-year Budget Authority (B\&R code $K A)$.

bincludes \$20.4 MR\&D funds from prior fiscal years.

\section{SSC Solenoidal Detector Collaboration}

The international collaboration on a solenoidal detector system for the SSC is directed toward exploring the rich domain of high-energy physics to be opened by the SSC. The initial goals include the discovery of new heavy bosons and critical elements of the standard model, including the Higgs particle and the top quark; investigating electroweak symmetry breaking and supersymmetry; and exploring quark and lepton substructure and other new phenomena. The requirements of a detector system to achieve these goals includie efficient lepton identification, isolation, and energy resolution; reconstruction of hadronic jet; and determination of missing transverse energy. A solenoidal detector system with high-resolution vertex measurement capability, outstanding tracking, well-understood calorimetry, and good calibration and monitoring can meet these requirements and achieve the physics goals.

The Laboratory has a leading role in the Solenoidal Detector Collaboration, which has emerged as an outgrowth of involvement in detectors worldwide, hosting the SSC Central Design Group, participation in work- 
View of interaction hall of the Solenoidal Detector showing an endcap calorimeter being lowered into the hall. This would occur at approximately month 25 of the installation schedule.

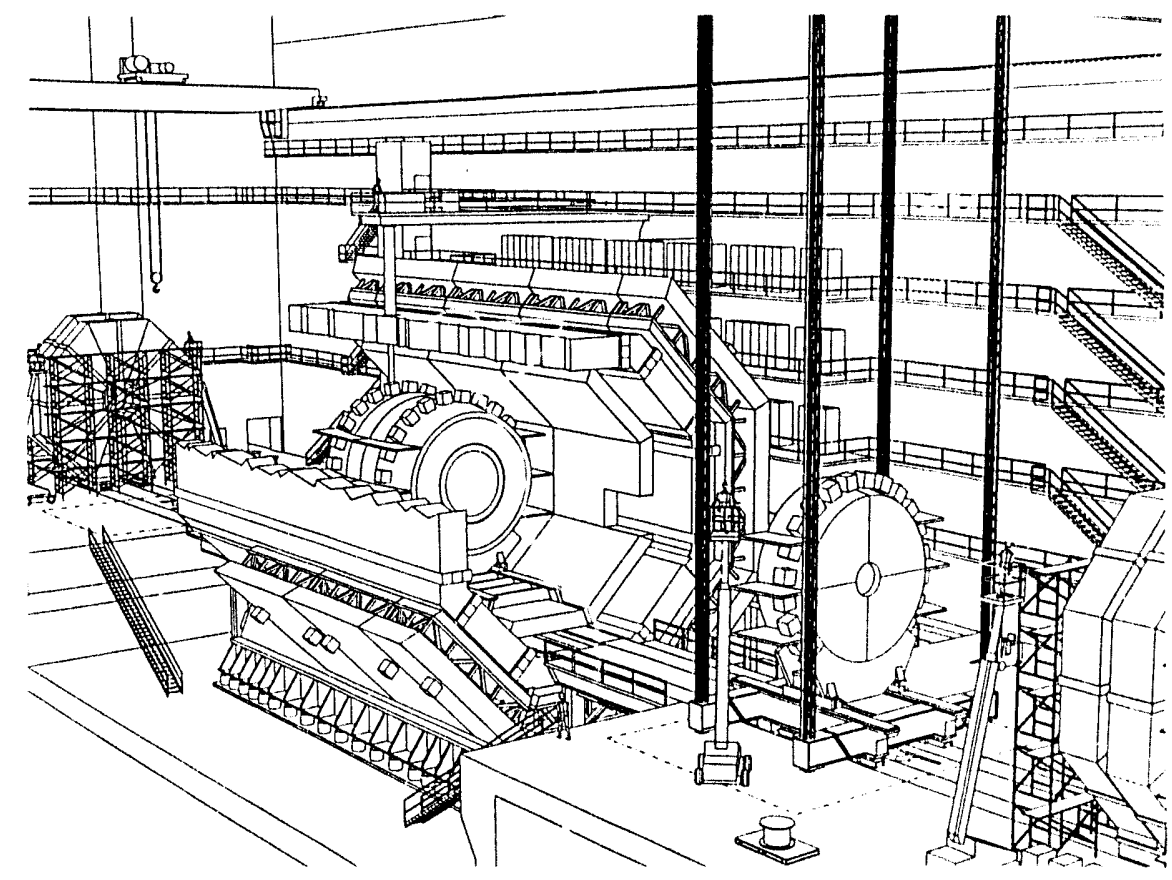

shops on SSC detector systems, and in developing the challenging technologies required for the high-rate environment of the SSC. This collaboration now comprises more than 1000 scientists representing more than 100 institutions from throughout the world. The collaboration spokesperson and the deputy project manager are at LBL, the project manager is at the SSC Laboratory, and the deputy spokespersons are from Fermilab, KEK in lapan, and the University of Pisa in Italy. Technical organization and design activities have begun for calorimetry design; computing and analysis software; electronics, data acquisition, and triggering systems; muon systems; superconducting magnets; and tracking systems; as well as overali detector integration and physics performance.

LBL has the established physics base to carry out its leadership and techni$\mathrm{cal}$ roles. Additional administrative and engineering resources are required to design the detector components, to develop the necessary subsystem prototypes, and to achieve the necessary infrastructure to complete the design for a construction start in FY 1994. LBL is responsible for the design and fabrication of major components of the SDC detector, generally in collaboration with other university and laboratory groups. These responsibilities include the endcap electromagnetic calorimeter, calorimeter electronics, silicon tracker modules and electronics and data acquisition and trigger systems. The cost profile identified below is an estimate of L.BL's component of the SSC detector efforts including all required $\mathrm{ES} \& \mathrm{H}$ costs.

SSC Solenoidal Detector Collaboration Resource Requirements $(\$ M)^{\text {a }}$

\begin{tabular}{lccccccc}
\hline Category & 1994 & 1995 & 1996 & 1997 & 1998 & 1999 & Total \\
\hline Operatingh & 3.0 & 3.0 & 3.0 & 3.0 & 3.0 & 3.0 & 18.0 \\
Construction & 0.0 & 0.0 & 0.0 & 0.0 & 0.0 & 0.0 & 0.0 \\
\hline
\end{tabular}

"Preliminary estimate of LBL Budget Authority (FY 1990) dollars); (B\&R code K.3)

"Resources are for detector fabrication, primarily equipment. 


\section{Relativistic Heavy-Ion Collider Program}

$L B L$ has played a seminal role in defining the forefront of relativistic heavy-ion physics since the field's inception and continues to maintain its leadership role. The Relativistic Heavy-Ion Collider (RHIC) is now under construction at Brookhaven National Laboratory (BNL), and LBL is the lead laboratory for the first approved RHIC experiment, STAR (Solenoidal Tracker At RHIC). LBL's Relativistic Nuclear Collisions Group is providing a focus for these RHIC activities. With 42 physicists, engineers and technicians (including the Spokesperson and Project Director) from LBL working on this experiment, the STAR collaboration now consists of 210 physicists and engineers from 28 institutions internationally.

The goal of the experiment is to study particle production at

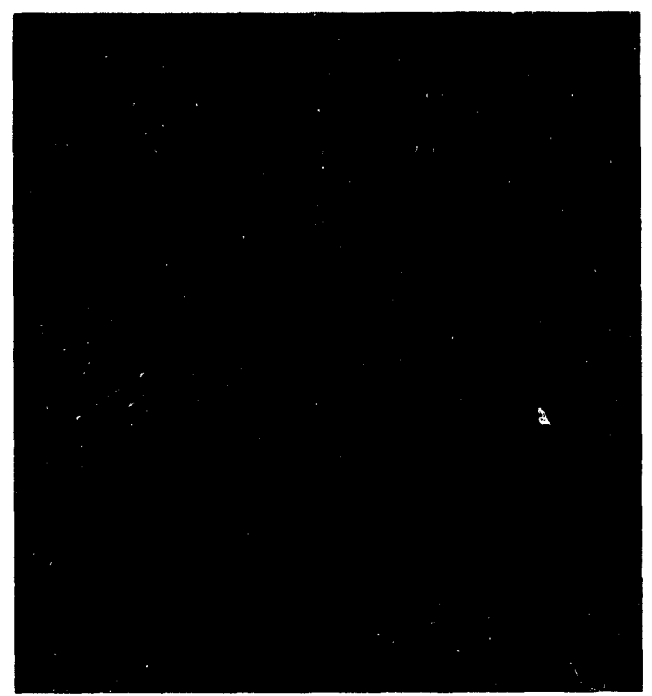
midrapidity on an event-by-event basis to identify the phase transition from normal nuclear matter to quark matter. An event-by-event measurement of the produced particles at midrapidity provides the opportunity to select on events with extreme values of temperature (particle spectrum), flavor (strangeness content), shape (particle momenta), and size (two-particle correlations). Furthermore, the study of high pt particle production as a function of energy and mass of the colliding system may also be an attractive experimental approach to identify the presence of quark matter. Hard-scattered partons, which are precursors of high $p_{t}$ particles and jets, are predicled to be sensitive to the medium through which they propagate and are directly calculable in quantum chromodynamics. The experiment will consist of a Time Projection Chamber (TPC) and silicon vertex tracker located inside a solenoidal magnet to provide tracking, momentum analysis and particle identification of charged particles. The trigger detector systems include a central trigger scintillator barrel around the TPC, vertex position detectors near the beamline just outside the magnet, and calorimeters located in the region

Charged particle tracks reconstructed in the STAR Time Projection Chamber from a simulation of a head-on collision of two silicon nuclei at a center-of-mass energy of $200 \mathrm{GeV}$ per nucleon pair. The STAR Time

Projection Chamber, which has been designed to analyze collisions of the heaviest nuclei at the Relativistic Heavy Ion Collider, will track approximately 3000 charged particles per event in head-on collisions of gold nuclei. of the beam insertion magnets to selectively veto events according to the number of spectators. An electromagnetic calorimeter to trigger on transverse energy and measure jet cross sections, a time-of-flight system surrounding the TPC for particle identification at higher momenta and external time projection chambers outside the magnet to extend the pseudorapidity coverage are anticipated as upgrades.

A detector R\&D program is currently underway to provide the detector and data acquisition developments required to support the experiment. Projects now receiving support from RHIC detector R\&D funds administered by BNL include TPC improvements for measurements at the high track densities anticipated at RHIC, integrated electronics for advanced detectors, and development of a silicon vertex tracker. These projects and the proposed STAR program are being developed in compliance with DOE Orders and all other applicable ES\&H requirements. LBL expects this R\&D effort and the detector engineering to continue over the next few years.

Relativistic Heavy-Ion Collider Program Resource Requirements $(\$ M)^{\mathrm{a}}$

\begin{tabular}{lrrrrrrr}
\hline Category & 1994 & 1995 & 1996 & 1997 & 1998 & 1999 & Total \\
\hline Operating & 1.5 & 2.5 & 4.0 & 5.0 & 6.5 & 7.0 & 26.5 \\
Construction & 4.8 & 3.1 & 1.4 & 1.0 & 0.8 & 0.5 & 11.6 \\
\hline
\end{tabular}

"Preliminary estimate of LBL Budget Authority (FY 1992 dollars), including equipment (B\&R code $K B)$. 


\section{IsoSpin Laboratory}

A broad base of nuclear scientists has recommended that the North American nuclear physics community should pursue the construction of a dedicated, flexible, radioactive nuclear beam facility that would provide intense beams of nearly all elements for a program of scientific studies in nuclear structure, nuclear reaction dynamics, astrophysics, high-spin physics, nuclei far from stability, material and surface science, and atomicand hyperfine-interaction physics. This international initiative has tentatively been given the name IsoSpin Laboratory (ISL) to underscore the key feature of this new physics tool.

The advent of unstable, radioactive nuclear beams in this decade may rival in importance the development of heavy-ion beams in the 1960 s. These beams remove the restraint to the natural neutron to atomic weight ratio of stable beams in nuclear, astrophysical, atomic, and material science experiments and add the new dimension of isospin to the two traditional dimensions of nuclear spin and temperature.

Elsewhere in the world, facilities utilizing the fragmentation of heavyion projectiles are in operation and others are planned. They produce nuclear beams in the energy range of tens of $\mathrm{MeV} /$ nucleon to $\sim 1000 \mathrm{MeV} /$ nucleon. For the new North American project a complementary approach to radioactive beam production has been chosen. The ISL is based on the coupling of two accelerators: the first to deliver a high-current light-ion beam to a thick spallation or fission target and the second to accelerate the emanating radioactive species with excellent beam qualities. New technical developments have made possible the intensities and purities required for meaningful experiments. The key advances are in high-current light-ion machines, new ion sources, radio frequency quadrupole pre-accelerators,

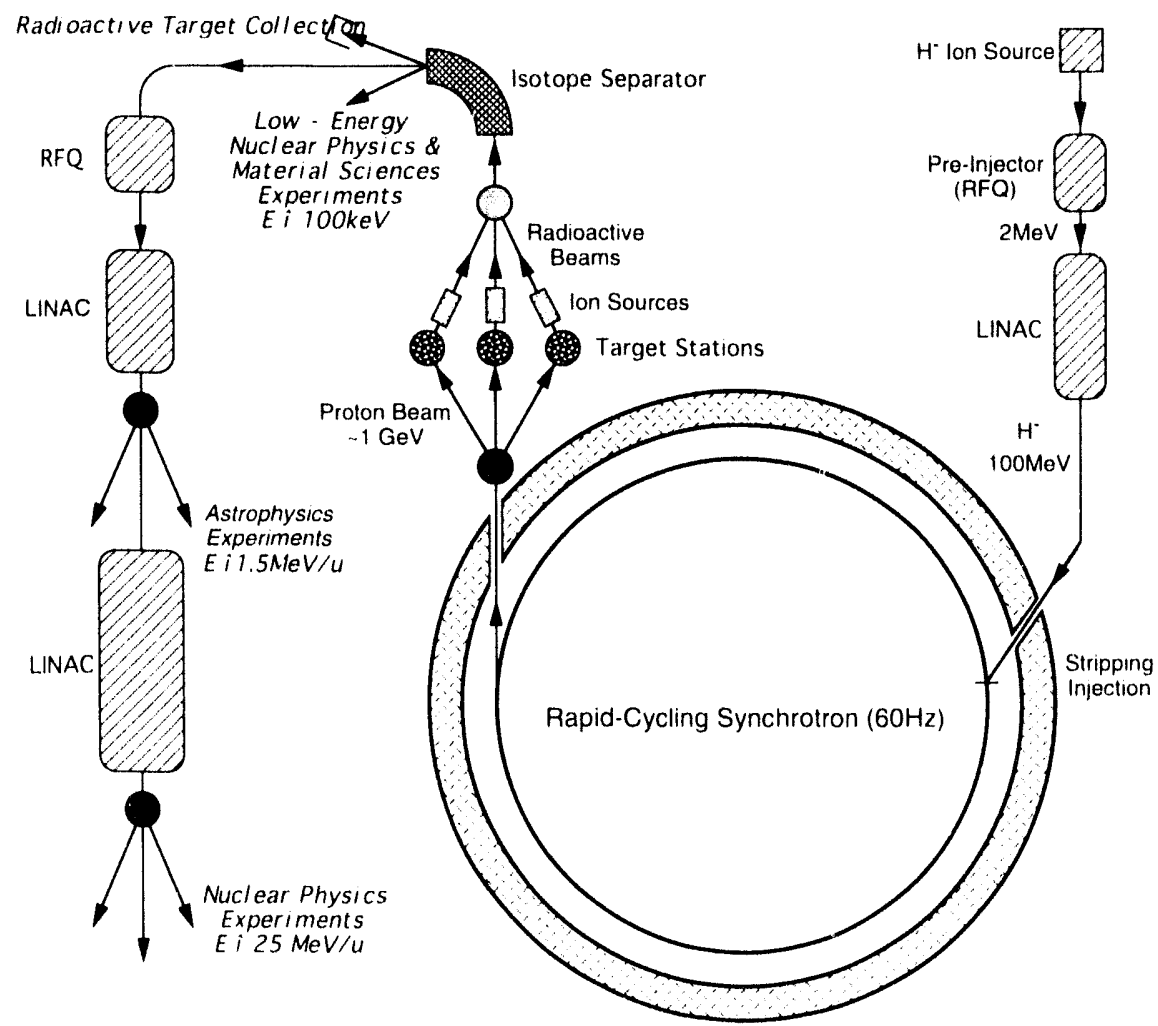

A schematic drawing of one option for implementing the IsoSpin Laboratory utilizing a rapid cycling synchrotron with a LINAC injector. 
low beam-oscillation accelerating structures, and superconducting linear accelerators. A number of these technologies have been pioneered at LBL and will be incorporated in the proposed IsoSpin Laboratory. The creative experiments under consideration will have to be highly efficient and insensitive to background radiation because of the inherently low-intensity radioaclive beams.

Several options for implementing the ISL, possibly at $L B L$, are being studied. One of the most attractive seems to be a rapid cycling synchrotron to serve, together with a new 70-100 MeV LINAC injector, as the primary beam accelerator. A proton energy of $1 \mathrm{GeV}$ and a current of $200 \mu \mathrm{A}$ would be obtainable, fully meeting ISL requirements. This would be followed by several target stations and a heavy-ion LINAC for the post-acceleration of the radioactive beams.

The ISL offers exciting physics prospects with a new understanding of nuclear structure and reaction dynamics, astrophysics, materials science, and atomic physics. LBL expertise in the underlying physics and machine design and development are coupled with excellent site characteristics and infrastructure. Existing expertise in environmental protection, health and safety for handling beam systems and isotopes couples well to the facility, together with a complete program for full documentation for NEPA and CEQA, if applicable, and health and safety procedures. Budget planning for the facility is under way; the following costs are for an initial design and planning program.

IsoSpin Initiative Resource Requirements $(\$ M)^{\mathrm{a}}$

\begin{tabular}{lccccccc}
\hline Category & 1994 & 1995 & 1996 & 1997 & 1998 & 1999 & Total \\
\hline Operating & 2.0 & 2.0 & 2.5 & 3.5 & 3.5 & 3.5 & 17.0 \\
Construction & 0.0 & 0.0 & 0.0 & 0.0 & 0.0 & 0.0 & 0.0 \\
\hline
\end{tabular}

"Budget to be determined for the facility.

\section{OFFICE OF HEALTH AND ENVIRONMENTAL RESEARCH}

\section{Human Genome Laboratory}

LBL was designated as a center for human genome studies as part of DOE's important role in the national effort to physically map and sequence the human genome. This national program will contribute significantly to understanding, diagnosing, and preventing hereditary and environmental diseases. LBL's Human Genome Center now requires the necessary laboratory facilities to conduct the research and development needed to map and sequence the human genome and to analyze the resultant complex genomic data. Programmatic elements to be conducted at the Laboratory would include:

- Mapping, cloning, and sequencing-develop new methods that will accelerate the speed of constructing large-scale restriction maps, ordered libraries, and completed DNA sequences of large regions of the genome. Immediate goals are to use the polymerase chain reaction to automate many of the steps required in DNA mapping and sequencing and to test new schemes for efficient large-scale mapping and sequencing for multiple genomes. 
An engineer at LBL's Cienome Center coperates a roboth that has been programmed to make copies of large numbers of plates of bacteria or yeast colonies dlibraries). Librarie's provide the basic IONA in small packages for sequencing and mapping the genome. The linear system permits the robot to handle many more samples than the original robot which could only work in a haltcircle. The system involves not only the stacks of multiwell plates (background, right), but also a sterilizer foreground, right for the multipin tool held in the robot arm.

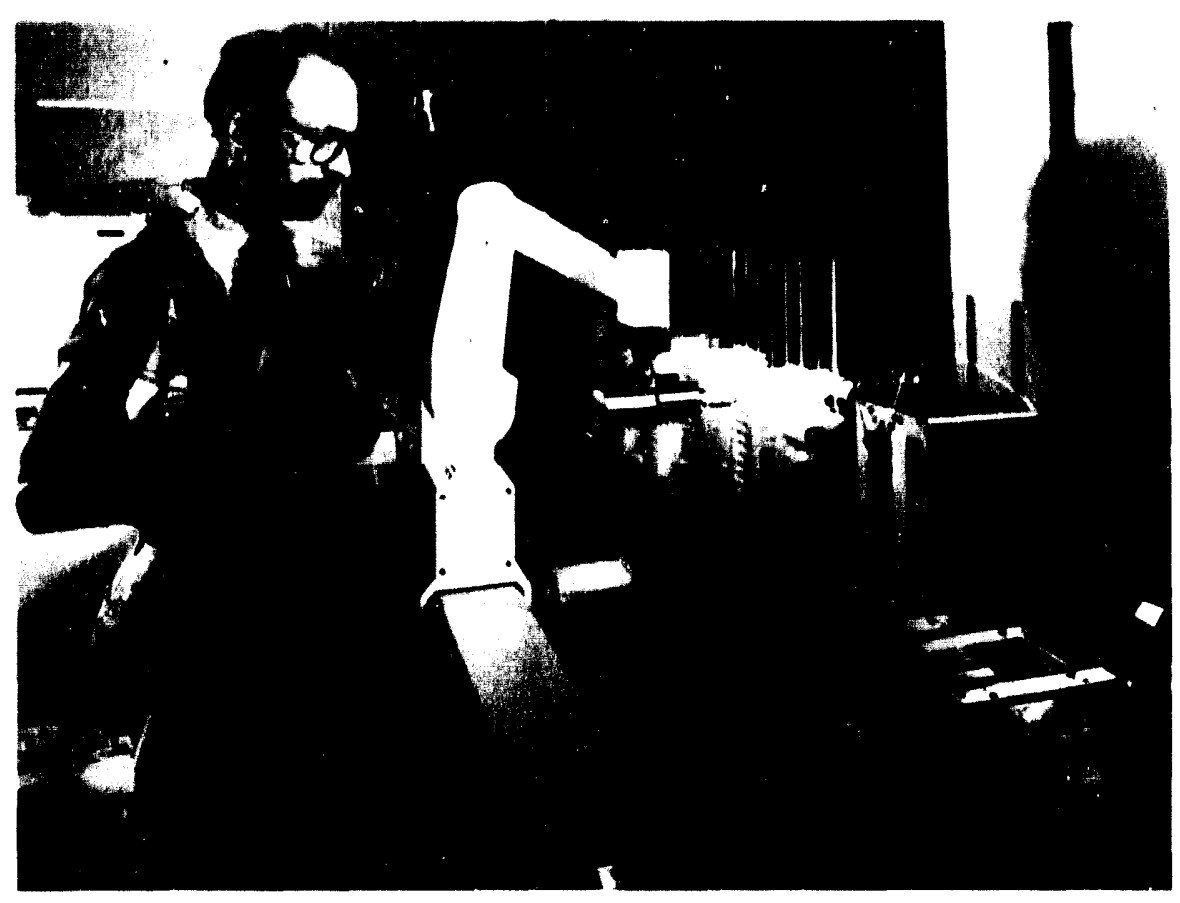

- Information systems-develop computational tools needed to analyze the mapping, cloning, and sequencing data generated from genome research at $L B L$ and throughout the scientific community and to provide computational foundations for the Human Genome Project. Develop novel data-management techniques needed for map and sequence data and laboratory information management. Investigate and implement methods for DNA fragment overlap detection, map assembly, and sequence and pattern matching;

- Instrumentation-develop innovative techniques in instrumentation and automation to accommodate the size and complexity of the experimental procedures. In addition to improving existing methods, emphasis will be placed on developing advanced techniques for handling large numbers of samples, e.g., large numbers of polymerase chain reactions, large numbers of oligonucleotides, etc. Methods for direct imaging of electrophoresis gels using modern detectors or optic al and ultraviolet imaging systems, including those based on chemiluminescence and fluorescence, will be explored, as will methods of manipulating, dissecting, and sequencing individual DNA molecules; and

- Structural and functional interpretation-interpret DNA sequence to identify transcriptional promoters and terminators, splice sites, reading frames, and protein binding sites; perform st:uctural analyses to predict unusual DNA structures relating to DNA regulation and RNA transcription; and relate these structural and functional elements to biological functions.

The program plans are being developed with close review and guidance from the (Office of Health and Environmental Research (OHER), with other national laboratories, and with the Life Sciences and computer Sciences Departments at UC: Berkeley and other UC campuses. LBL expects the research and development efforts to grow significantly during the next 


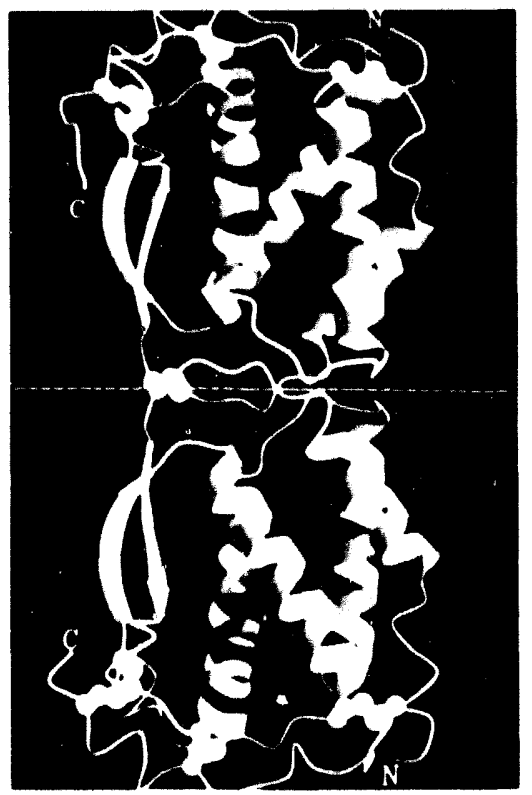

The birekbone structere of the mac rophonge-crolony stimulating

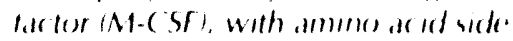

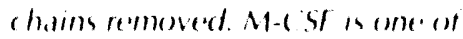
several seghaling molectules that regulalle grewth and ditterentlalterm of bethered and whete blesed cells. In clinical trath. M-c st has shown promere in the correctem at blesert cell defic iencies and an all agerell

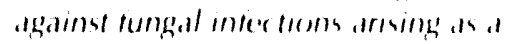

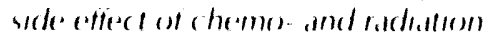
theralpy several years. The major experimental activities at that time will be conduc led in an essential new fac ility, the Human Cenome Laboratory, to be constructed in the L ife Sciences Functional Area of the Laboratory. This new laboratory will consist of $3850 \mathrm{gsm}(41.500 \mathrm{gsf}$ of light-laboratory space with functions dedicated to the conduct of mapping, cloning, and sequencing activities along with integrated instrumentation, computation, and all required ES\& $1+$ support facilities and building design elements. A plan for recommended operational safety procedures has been generated, and an Environmental Assessment under NEPA and Environmental lmpact Report under (EC $2 A$ are in preparation. As more is learned about the map of the human genome, the efforts will be integrated with fundamental studies in molecular genetics and gene expression.

Human Genome Laboratory Resource Requirements $(\$ M)^{*}$

\begin{tabular}{lrrrrrrr}
\hline Category & 1994 & 1995 & 1996 & 1997 & 1998 & 1999 & Total \\
\hline Operating & 9.0 & 10.0 & 12.0 & 12.0 & 12.0 & 12.0 & 67.0 \\
Construction & 2.2 & 15.6 & 5.9 & 1.0 & 0.0 & 0.0 & 24.7 \\
\hline
\end{tabular}

"Preliminary estimate of actual-year LBL. Budget Authority (B\&R code- KP).

\section{Structural Biology Initiative}

New applications of advanced imaging, diffraction, and spectroscopy techniques will greatly strengthen DOE's emerging national program in structural biology. A structural biology research program is being formulated at LBL to develop the potential structural biology resources at the Advanced Light Source. The ALS will offer major new resource opportunilies for life sciences research supporting several facilities in emerging areas of scientific emphasis:

- X-ray microscopy-to investigate tissues, cells, and organelles in nearnative states at a resolution that may approach macromolecular dimensions. Among the benefits of $x$-ray microscopy at the ALS will be element-specific imaging and the ultimate possibility of threedimensional imaging.

- X-ray crystallography-- to conduct static and dynamic analysis of macromolec ular architeclure with precise wavelength luning. The facility addresses the high demand for beam time with a multiuser configuration and user-friendly design and operation.

- X-ray spectroscopy - 10 determine biochemical properties at high spatial and temporal resolution within cells and organelles. Many experiments will explest the capac ity to control the polarization of synchrotron radiation.

The struc tural biology programs at the Al.S will be foc used on experimental stations, mitially at the ends of the three beamlines. The first beamline, from an undulator source of ultrabright soft $x$-rays, will illuminate two x-ray mic rese ope stations, one available for biolegical microscopy and the other for developing advane ed microscopy techniques. The second beamline, from a wiggler source of both soft and hard $x$-rays, will branch into several experimental areas inc luding $x$-ray crystallography and spectroscopy. The third beamline will come from a polarized wiggler for applica- 
tions in structural biology and the materials sciences. The majority of the operating (and eouipment) funds identified below will be for fabrication and development of these beamlines. The beamline and equipment components will include all safety systems, interlocks, and biohazards controls in compliance with DOE Orders and regulations; and appropriate safely procedures and manuals will be prepared prior to final design or operation. The supporting laboratories will be constructed in the second floor of Building 6 and in the adjacent Building 80, as part of the AL.S Structural Biology Support Facilities. A categorical exclusion under NEPA and categorical exemptions under CEQA will be prepared for associated modifications to Building 80 .

These ALS projects will be coupled to other structural biology expertise at L.BL. to initiate new collaborations and to participate in the national scientific program. Other relevant strengths at LBL include electron crystallography, high-voltage electron microscopy, NMR spectroscopy, limeresolved fourier-transform infrared spectroscopy, and scanning tunneling microscopy.

Structural Biology Initiative Resource Requirements (SM) d

\begin{tabular}{lccccccc}
\hline Category & 1994 & 1995 & 1996 & 1997 & 1998 & 1999 & Total \\
\hline Operating & 8.0 & 5.5 & 4.5 & 4.1 & 4.1 & 4.1 & 30.3 \\
Construction & 0.6 & 4.7 & 2.6 & 0.0 & 0.0 & 0.0 & 7.9 \\
\hline
\end{tabular}

"Preliminary estimate of actual-year LBL Budget Authority (B\&R code KP).

\section{Global Change Research Program}

OHER is participating in national and international efforts to understand complex and interdependent global environmental processes, including global climate change and its potential consequences. LBL scientists, through laboratory, field, and theoretical research, have contributed to the existing concepts on global and regional atmospheric phenomena and are participating in DOE's planning processes.

LBL is developing an interdisciplinary program to investigate the processes that lead to changes in the physical and chemical characteristics of the atmosphere, to provide the information to global climate modelers, for example, on cloud properties and other characterizations, and to assess potential regional ecosystem changes. The effort involves collaborations with several divisions at LBL, various UC campuses, and Lawrence Livermore National Laboratory (LLNL.) to use most effectively a breadth of research capabilities. The effort will benefit from instrumentational and computational capabilities developed at LBL and LLNL, such as the Cloud Chamber Facility at LBL. The effort is being developed in close conjunction with LBL.'s national and internatiunal policy-related studies on greenhouse gas issues sponsored by DOE's Office of Policy, Planning and Analysis. Areas of initial interdisciplinary research include:

- Laboratory, chamber, and field studies of atmospheric radiation properties including the physics and chemistry of cloud processes such as the effect of natural and anthropogenic nucleating particles on cloud optical characteristics. 
- Almospheric ecosystem interactions, such as increased UV radiation from ozone depletion, buildups of $\left(\mathrm{C}_{2}\right.$, methane and other gases/ particulates, and temperature changes, which all play a potential role in the modification of ecosysterns.

- Quantilative understanding of the sources and sinks of carbon in the oceans, including primary production, ocean mixing, transport of heat and carbon, gas hydrates, and isotopic composition of ocean sedi. ments.

The effort is coordinated by LBL's Center for AImospheric and Bio. spheric Effects of Technology. Resource requirements, with necessary ES\&H operational and equipment needs projected for the program, follow. As research proposals under this program are developed, they will be reviewed for NEPA and CEQA compliance requirements.

Global Change Research Program Resource Requirements (SM)“

\begin{tabular}{lccccccc}
\hline Category & 1994 & 1995 & 1996 & 1997 & 1998 & 1999 & Total \\
\hline Operating & 2.5 & 3.0 & 3.5 & 3.5 & 3.5 & 3.5 & 19.5 \\
Construction & 0.0 & 0.0 & 0.0 & 0.0 & 0.0 & 0.0 & 0.0 \\
\hline
\end{tabular}

"Prelliminary estimate of LBL acturi year Budget Aurhorily (BXR code KP).

\section{National Biomedical Tracer Facility}

Based on LBI.'s tradition of radiobiology and isotope chemistry, the Laboratory is supporting DOE's Health and Environmental Research program to conduct isotope-based research and to provide tracer isotope resources for the nation's biomedic al research community. The National Biomedical Tracer Facility will maintain U.S. research infrastructure for stable and radioactive tracers that are of vital need in biomedical research including metabolic studies, diagnostic imaging, and other applications.

The National Biomedical Tracer Facility will benefit from the Laboratory's strengths in accelerator systems design and engineering, biomedical applications of isotopes, and radiopharmaceutical chemistry. The facility would be based on a proton accelerator with a maximum energy of $100 \mathrm{MeV}$ and a current of 500 to $750 \mu \mathrm{A}$. The facility would include isotope processing and radiochernic al laboratories and supporting infrastructure. Imy ortantly, the facility will benefit from the technical expertise at LBL, including isotope preparation and handling, radiochemical systems, accelerator design, engineering, and EHXS operations. The facility can ulilize many of the Laboratory's existing resources, including utilities, shielding, and some of the components of the recently closed Bevalac acceleraters.

The National Biomedical Tracer facility will provide critic ally important resources to the biomedical community and will benefit from LBL.'s long tradition of radiobiological research that began with the inception of the cyclotren at I.BL. The facility will be operated in close conjunction with the biomedic al industry and will afford valuable graduate student, postdoctoral, and industry training programs. The facility will be operated as a controlled fac ility in compliance with all envirenment, heallh, and safety regulations. Invironmental doc uments will be prepared in full compliance with NEPA and Cl(2). 
National Biomerlic al iracer facility Resenurce Requirements (SM)"

\begin{tabular}{llllllllll} 
Collegory & 1494 & 1995 & 1996 & 1997 & 1998 & 1999 & Tot.1 \\
\hline Operating & 0.1 & 0.1 & 0.3 & 1.5 & 2.0 & 2.0 & 6.0 \\
Construction & 0.7 & 3.8 & 19.0 & 12.0 & 11.0 & 1.5 & 40.0 \\
\hline
\end{tabular}

"Preliminary entimate actual year Budget Authorily (BXR conke KP)

\section{ENERGY EFFICIENCY AND RENEWABLE ENERGY}

\section{Advanced Energy Design and Operation Technologies}

LBI. and other DOE national laboratories are engaged in a continuing research effort to improve energy efficiency in buildings. LBL's contributions to DOE's Advanced Energy Design and Operation Technologies (AEDOT) Initiative would develop innovative methods to incorporate arlvanced energy-efficiency concepts directly into building design and operation. These concepts can provide an additional energy savings of approximately $50 \%$ beyond the efficiencies already achieved. The design system would integrate new computer-aided design (CAD) systems with sophistic ated graphics, expert systems, and new energy-simulation models. Activities underway at LBL coupled to the initiative include:

- DOE-3, the next-generation whole-building energy-simulation program;

- Computer-generated visualization of interior lighting and daylighting;

- Studies of innovative building technology and systems such as win. dows, lighting, ventilation, and indoor air quality:

- User-friendly interfaces, (AD)/CAM systems, and large databases; and

- Exploratory research on expert systems for building design and opera. tion.

AEDO I software would link energy and nonenergy issues in buildings-to integrate quantitative (e.g., energy consumption) and qualitative (e.g., aesthetics) aspects of design, including considerations of occupant productivity. The initiative is intended to incorporate energy efficiency, building structure, and other design elements directly into architectural systems. Advanced simulation and imaging lechnology would provide engineering accuracy and visual realism. The complete building cycle would be ad. dressed, providing novel feedback to integrate design, construction, occupancy, maintenance, and economics.

An ongoing challenge is the transter of building research results to the building industry, since energy effic iency is offen treated as separate from the architectural design. The envisioned system would become a vehicle for the transfer of tee honology for almost all of $D$ OCE's research prodects to the design and construction industry. Reseurce requirements inc lude ESAH and distributed data manogerement costs. Specifie research propessals will be reviewed for NEPA and C LCA compliance recpuirements 
Advanced Energy Design and Operation Technologies

Resourre Requirements (SM)"

\begin{tabular}{llllllll}
\hline Category & 1994 & 1995 & 1996 & 1997 & 1998 & 1999 & Total \\
\hline Operating & 2.0 & 2.0 & 2.0 & 2.0 & 2.0 & 2.0 & 12.0 \\
Construction & 0.0 & 0.0 & 0.0 & 0.0 & 0.0 & 0.0 & 0.0 \\
\hline
\end{tabular}

"Preliminary estimate of LBL actual year Budget Authorily (fY 1992 dollars): (BRR coxte EC).

\section{DOMESTIC AND ENERGY POLICY}

\section{Assisting Development of Energy Practices and Technolo- gies}

The Assisting Development of Energy Practices and Technologies (ADEPT) Initiative has helped to establish a new program at DOE that provides assistance to developing and transitional countries in their choice and application of energy technologies. The program supports technology choices that-when compared with lypical current technology-produce or use energy more efficiently, cause reduced levels of emissions of pollutants and greenhouse gases, and are economically competitive or superior on a lifecycle cost basis.

The new program consists of seven elements: (1) energy technology adaptation, (2) demonstrations, (3) key country programs (including China and Indial, (4) one-stop shopping network, (5) training, (6) applied R\&D, and (7) institution building in developing countries and Eastern Europe. Initial work on the program has begun within the Office of Policy, Planning and Education during FY 1993.

ADEPT will be especially valuable in responding to needs of developing countries, and the former Soviet Union and Eastern Europe, for technical assistance toward limiting greenhouse gas emissions in a highly costeffective manner. It will also provide important benefits in reducing future global oil demand and in helping to establish international markets for U.S. energy lechnology. This program is an important United States contribution 16 support recommendations made by the Intergovernmental Pand on Climate Change and the United Nations Conference on Environment and Development. The program will be conducted consistent with applicable DCE orders and regulations. The resources indic ated below are for LBL.'s activities for the national DOE program. Specific research proposals will be reviewed for NEPA and CEQPA compliance requirements.

Assisting Deployment of Energy Practices and

Technologies Resource Requirements (\$M)"

\begin{tabular}{lccccccc}
\hline Category & 1994 & 1995 & 1996 & 1997 & 1998 & 1999 & Total \\
\hline Operating & 0.8 & 1.2 & 1.5 & 2.0 & 2.2 & 2.4 & 10.1 \\
Construction & 0.0 & 0.0 & 0.0 & 0.0 & 0.0 & 0.0 & 0.0 \\
\hline
\end{tabular}

"Proliminary estimate of I BL actual year Budget Authority (BAR code. PE). 


\section{FUSION ENERGY}

\section{Induction Linac Systems Experiments}

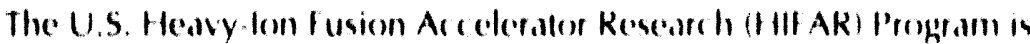
building the research database to assess and develeph heavy in an accolerators as drivers for an inerlial.fusion energy source for commercial power genera. tion. The results of the suc cessful Single-Beam Transpont Experiment and the Multiple. Beam Experiment provide encouragement and justification to conduct larger, more complex experiments; however, these experiments do not demonstrate all features of a full-scale fusion driver. Yet lo be demonstrated are the components for beam merging with minimal phase-space density dilution, Iransition from electrostatic to magnetic focusing. Iranspont in bending and focusing magnets, drift compression, and foc using onto a small target. To demonstrate these important beam components and manipulations. LBL proposes to build a new accelerator known as ILSE: (Induction Linac System Experiments).

The II.SE Program has two parts, an accelerator construction project and a series of experiments to be performed with the accelerator. The ILSE accelerator and the associated experiments are designed to test nearly all the features of a fusion driver. Using a 2.MeV injector, ILSE will accelerate ions such as $\mathrm{Ne}^{+}$or $\mathrm{K}^{+}$to $10 \mathrm{MeV}$, after which they will be transported and focused to a small spot. To minimize cost, the ILSE accelerator is shorter and has fewer beams than a full-scale driver, but the beams will have the same diameter and line charge density as driver beams. ILSE will therefore test much of the important driver physics at full scale. The ILSE project also provides a pathway for the development of the pulsed-power ter hnology with applicability to the production of pulsed neutrons (see Section 5). Incremental costs of ILSE hardware above the base HIF AR Proghram are indicated in the following table, which assumes a construction project start in Fy 1995. Additionally, the Heavy-Ion Fusion base program will continue theoretic al studies and small experiments required to imvestigate fundamenhal aspects of heavy-ion fusion physics. It also will address the design of the II SE experimental program that will begin in FY 1999 and require a stupport

A plan verw of the ll se laboul in shewn. The llst expermentents wall study bedm combenings. bending.

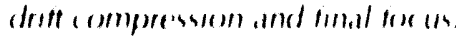

$B \log 518$
Drift

Compression Experiments

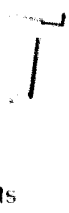

1

Blog 51

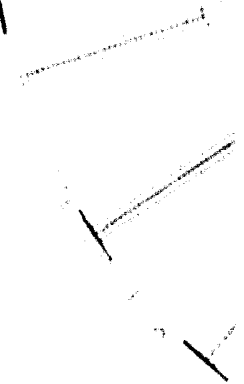




\section{Initiatives}

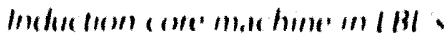

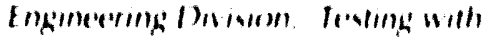

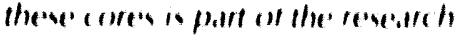

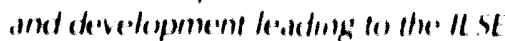
proment.

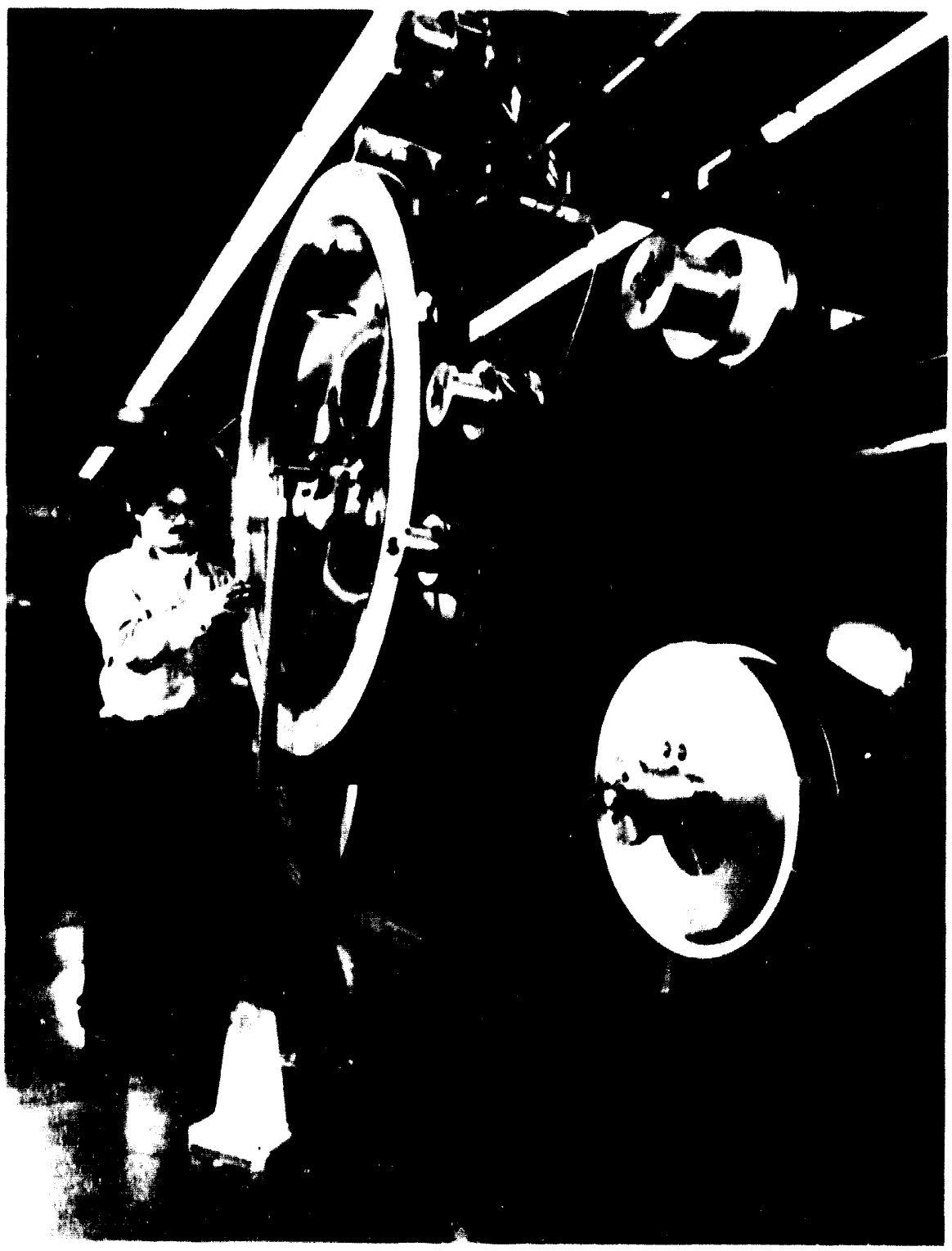

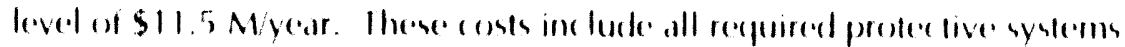

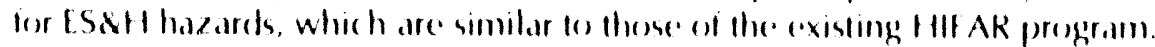

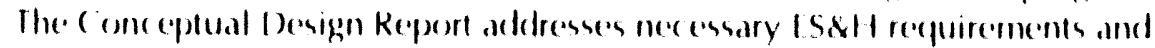
NIPACE(QA docomentalion).

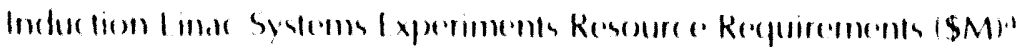

\begin{tabular}{|c|c|c|c|c|c|c|c|}
\hline C alegory & 1994 & 1905 & 1996 & 1997 & 1908 & 1909 & $|0|,||$ \\
\hline 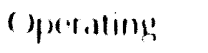 & 23 & 0.7 & 11.7 & 0.7 & 2.4 & 11.5 & 18,3 \\
\hline comstruclion & 0.0 & 8.1 & $1: 2$ & 14.1 & 9.6 & 0.0 & 44.11 \\
\hline
\end{tabular}

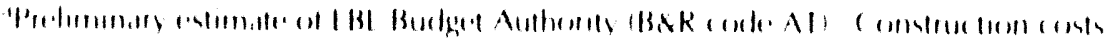

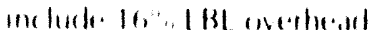




\section{Neutral Beam Test Facility for ITER}

Most proposals for future magnetic - fusion projects involve injertion of energetic neutral beams at high currents; the beams play a signific ant role in heating the plasma and in driving the foroidal current nonindustively in the steady state. LBL has traditionally been a leading center for aesign and development of these neutral-beam injection systems, inc liuding the shandardized Common Long.Pulse Source that has been incorporaled info all major U.S. fusion experiments.

The International themomuclear Lxperimental Reactor (ITRR), a pro. posed next-generation tokamak to be built by a multinational collaboration. may need neutral-beam systems of higher power and energy la total of 75 MW of $D^{\prime \prime}$ at $1.3 \mathrm{MeV}$ from 9 injectors) capable of operating continuously for periods as long as two weeks. Signific ant design challenges, many of which are already being addressed in the Magnetic Fusion Energy Progran at LBL, include negative-ion sources, accelerators, neutralizers, and a suitable test facility.

I.BL's role in ITER has been lo participate in a conceptual design for a neutral-beam system agreeable to all four participants the U.S., Curope, lapan. and the former Sovict Union) and to conduct supporting research and develop) ment in the areas of 1 ) ion sources and high-voltage de accelerators. IBL is now proposing a major ITER neutral-beam development project, including a new accelerator test facility for conducting a proof-of-principle accelerator demonstration. The resource requirements indicated below are the incremental fabrication and operating costs for the proposed facility. These costs inclucte all protective systems for ES\&H compliance. Environmental doc uments will be prepared in full compliance wilh NEPA and CEQ QA.

Neutral Beam Test facility for IrfR Resource Projections (SM)

\begin{tabular}{lccccccc}
\hline Category & 1994 & 1995 & 1996 & 1997 & 1998 & 1999 & Total \\
Operating & 0.1 & 4.2 & 8.0 & 2.7 & 4.6 & 1.4 & 21.0 \\
Comstruction & 0.0 & 0.0 & 0.0 & 0.0 & 0.0 & 0.0 & 0.0
\end{tabular}

"Preliminary astimate of LBL Budget Authority, including engupment (BAR code AT)

\section{ENVIRONMENTAL RESTORATION AND WASTE MANAGEMENT}

\section{Environmental Restoration Research and Development}

In support of the (office af Technology Developenent in 1)( )I's Inviron. mental Resteration and Waste Management program, and to facilitate the development of underlying se Ience conducted in the (offic es of Basic Inergy Sciences and Healih and Envirommental Research, I BI is develepping a moltidise iplinary research and develepenent progeram direc ted lo improving

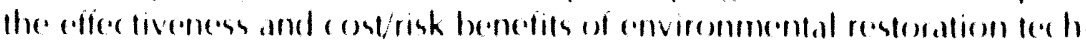
moleggies. The progham has lour components

- Improved charac ferization of subsurface enviromments, inc lueling beller medsurement of their hiolesgic al, chemic al, and phyase al propertices and

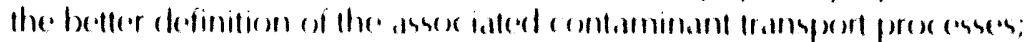


- Development of methods for assured containment and control of subsurface biological, chemical, and radiological contaminations;

- Development of advanced remediation technologies including instrumentation for cost effective measurements and treatrnent methods appropriate to complex and heterogeneous subsurface environments;

- Improved risk assessment and prioritization systems to allow better allocation of remediation funds.

The methodologies used in the program will include field testing and tracking contaminant fronts; developing descriptive and predictive mathematical models; characterizing heterogeneous underground systems; designing, demonstrating, and testing containment and cleanup systems at specific contaminant sites; and determining the underlying chemical, biological, and thermodynamic properties involved in mixed contamination. As program plans are developed, they will be reviewed for NEPA and CEQA documentation requirements.

Environmental Restoration Research and Development Resource Requirements $(\$ M)^{\mathrm{a}}$

\begin{tabular}{lccccccc}
\hline Category & 1994 & 1995 & 1996 & 1997 & 1998 & 1999 & Total \\
\hline Operating & 3.0 & 4.0 & 5.0 & 5.0 & 5.0 & 5.0 & 27.0 \\
Construction & 0.0 & 0.0 & 0.0 & 0.0 & 0.0 & 0.0 & 0.0 \\
\hline
\end{tabular}

aPreliminary estimate of LBL Budget Authority (B\&R code EX).

\section{WORK FOR OTHERS}

\section{Advanced Lithography Initiative}

Since the creation of the first integrated circuit in 1960, there has been an ever-increasing density of devices manufactured on semiconductor substrates. The VLSI (very large scale integration) era from the mid-70s to the present has seen chip densities increase from 100,000 transistors per chip to over 1 million per chip. The increasing device count was accompanied by a shrinking minimum feature size from $2 \mu \mathrm{m}$ in the late 70 s to less than $0.5 \mu \mathrm{m}$ in current 4 -megabit DRAMs. The challenge to continued U.S. industrial competitiveness in microelectronics is the development of techniques of lithography and pattern transfer at minimum feature sizes less than $0.25 \mu \mathrm{m}$. This will lead to chip densities of over $10^{9}$ transistors per chip. The advanced lithography program at LBL's Center for X-Ray Optics (CXRO) focuses on the enabling technologies essential for extreme ultraviolet (EUV) optical imaging systems. These systems will be required for $0.1 \mu \mathrm{m}$ features, and 1-Gbit DRAM's by the year 2000. CXRO's lithography program will concentrate on the development of EUV/SXR interferometry for at-wavelength (130 A) testing of optics, nanofabrication facilities for zone plates and reflective masks, general $x$-ray metrology and the necessary processing for pattern transier.

LBL's initiative responds to programmatic needs established by the Department of Defense to provide collaborative support for this emerging area of science, which supports essential U.S. technological capabilities. A 
A schematic diagram of the "atwavelength" interferometer for extreme ultraviolet optical systems. This technique is a critical tool for assessing improvements in pattern transfer capabilities of new reflective optical systems being developed in industry for extreme ultraviolet lithography of nano-electronic devices. The system will use coherent radiation available only at the ALS, and will be done in collaboration with Lawrence Livermore National Laboratory and their industrial partners.

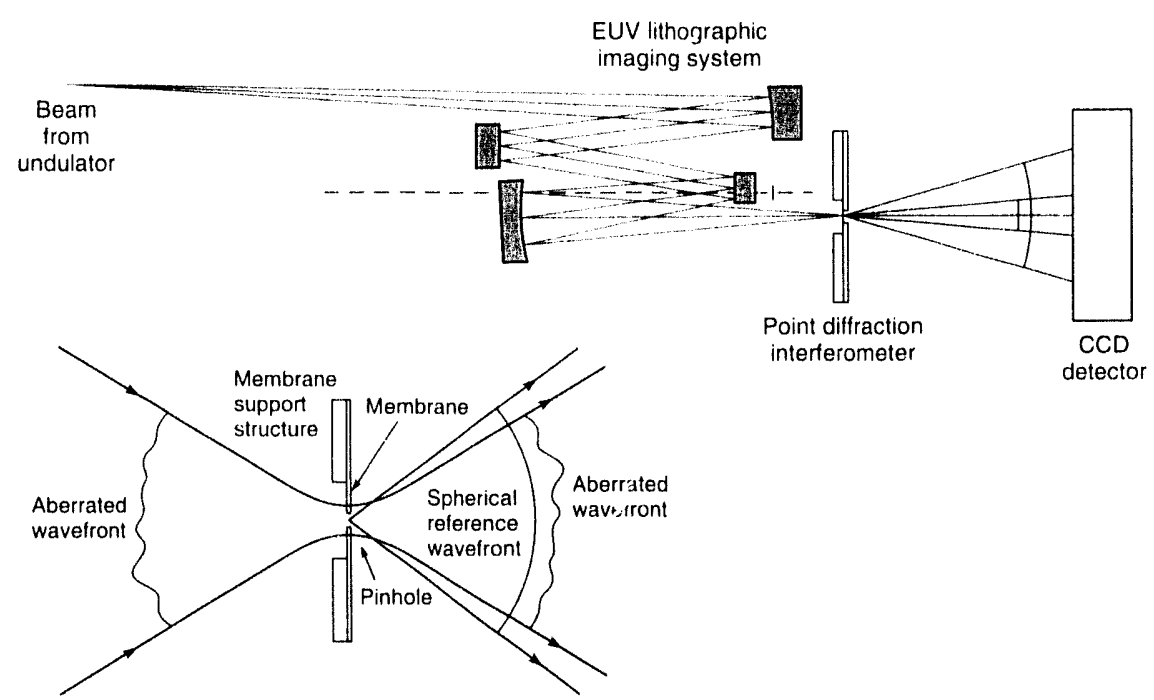

successful program requires an integrated consortium of industrial, university, and national laboratory scientists to break new scientific and technical ground well in advance of the competition and to train a new generation of scientists to carry the project to fruition in the 21 st century. The consortium will require dedicated laboratory research facilities with modern highbrightness partially-coherent $x$-ray sources, expertise in relevant $x$-ray optical techniques, an active student research program, and state-of-the-art equipment for the fabrication of nanometer-scale structures and the synthesis of new materials whose structures are controlled at the atomic level. Strong participation of a consortium of local microelectronics companies will ensure quick and effective transfer of all relevant technologies.

The ALS synchrotron storage ring will be a unique national resource to be utilized by the collaboration. Additional laboratory equipment dedicated to advanced lithographic research for microelectronics applications will be required. These facilities will include insertion devices, bend magnets, and beamline components; metrology stations to test all optical surfaces and coatings; and advanced EUV interferometers for testing optical surfaces and integrated optical systems and equipment that will support the fabrication of nanostructures and the synthesis of artificially structured materials. Specific initial elements are to include:

- A high-brightness coherent undulator beamline for at-wavelength interferometry

- A direct-write electron beam "nanowriter" for mask patterning and diffractive optics

- Component and system interferometry

- An EUV metrology bending magnet beamline

A continued investment would further the consortium efforts and lead to improved American competitiveness in this critical technological area. Costs include operational procedures and equipment design in compliance with DOE ES\&H orders and regulations. As proposals under this program are developed, they will be reviewed for NEPA and CEQA documentation requirements. 


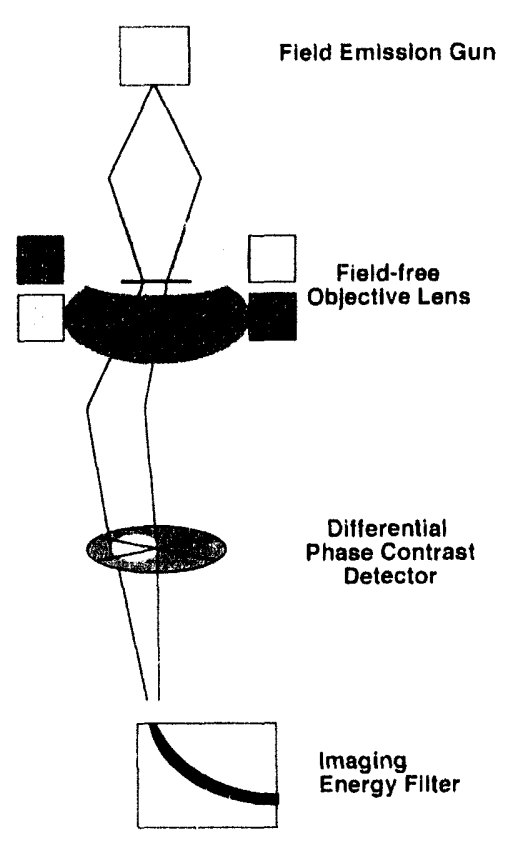

Conceptual schematic diagram of the proposed Magnetic Materials Microscope for field-tree imaging of magnetic, physical, and chemical microstructures at the nanometer scale of resolution. Current research into magnetic materials is limited by the electromagnetic field of the objective lens. Such research is essential for understanding permanent magnets, thin tilm magnetism, and other novel science areas.
Advanced Lithography Initiative Resource Requirements $(\$ M)^{\text {a }}$

\begin{tabular}{lrrrrrrr}
\hline Category & 1994 & 1995 & 1996 & 1997 & 1998 & 1999 & Total \\
\hline Operating $^{\mathrm{b}}$ & 15.0 & 15.0 & 8.1 & 0.0 & 0.0 & 0.0 & 38.1 \\
Construction & 0.0 & 0.0 & 0.0 & 0.0 & 0.0 & 0.0 & 0.0 \\
\hline
\end{tabular}

aPreliminary estimate of LBL actual year Budget Authority from DOD.

${ }^{b}$ Costs are primarily for equipment.

\section{Magnetic Materials Microscope}

The National Center for Electron Microscopy (NCEM) at LBL is an established user research facility for the electron optical characterization of materials at high resolution. Its complement of instrumentation includes the world's first Atomic Resolution Microscope, the nation's highest voltage electron microscope $(1.5 \mathrm{MeV})$ and soon one of a new generation of instruments capable of one-angstrom resolution. The facility is available for use by industrial, government-laboratory, and academic researchers from around the nation and the world.

NCEM has proposed a new instrument, the Magnetic Materials Microscope $(M M M)$, to be added to the facility. The MMM will permit micro-characterization of magnetic materials in a field-free environment. Its unique capability will provide new insights and advance ongoing research on magnetic materials.

Magnetic materials are at the heart of many modern technologies ranging from information recording and storage to electric motors and power generation. New magnetic effects that have recently been discovered in solid surfaces and multilayers have great potential for new technologies. However, present understanding of the microstructure of materials lags behind because the strong magnetic field of the focusing lens in an electron microscope interferes with the magnetic microstructure. The MMM will feature a field-free objective lens without compromising the high resolution typical for electron microscopes. This will allow unprecedented resolution in the direct quantitative characterization of the micromagnetic behavior of materials.

The location of the MMM at NCEM, with its established user base and its scientific and computing support, will ensure optimum access for scientific researchers nationwide. The Magnetic Materials Microscope Project will be implemented in full conformance with safety and health considerations. DOE and $U C$ will be requested to determine the appropriate NEPA and CEQA documentation for this initiative. The facility will be located adjacent to the existing High Voltage Electron Microscope and adequate user facilities and preparation areas are included in the project scope.

Magnetic Materials Microscope Resource Requirements $(\$ M)^{a}$

\begin{tabular}{lccccccc}
\hline Category & 1994 & 1995 & 1996 & 1997 & 1998 & 1999 & Total \\
\hline Operating & 6.0 & 0.0 & 0.0 & 0.0 & 0.0 & 0.0 & 6.0 \\
Construction & 1.0 & 0.0 & 0.0 & 0.0 & 0.0 & 0.0 & 1.0 \\
\hline
\end{tabular}

"Preliminary estimate of actual-year LBL Budget Authority. This is a preliminary estimate only and does not distribute funds among operations, equipment, or construction. 


\title{
5 SCIENTIFIC AND
}

\author{
Distinguish ourselves as a premier DOE multiprogram \\ national laboratory by performing research of the highest \\ scientific quality.... Our operational, administrative, and \\ technical resources will integrate seamlessly with the \\ research and engineering programs to make an $L B L$ that \\ works.
}

LBL Vision 2000

Lawrence Berkeley Laboratory research programs fortify the foundations of energy and environmental technology and will continue to be supported primarily from the Office of Energy Research (OER) and the Assistant Secretarial Offices of Energy Efficiency and Renewable Energy, Civilian Radioactive Waste Management, and Fossil Energy. The Assistant Secretary for Environmental Restoration and Waste Management continues to be important for supporting site-specific environmental restoration projects. In addition, other DOE offices and the Nuclear Regulatory Commission will support LBL programs. Work for Others (WFO) supports about one-fifth of the Laboratory's programs. This section summarizes current LBL research programs, including anticipated program trends. LBL's scientific and technical programs are conducted under strengthened environment, safety, and health guidelines for conduct of operations. Research facilities and programs are conducted to ensure the safety of all employees and the public, with environmental and safety management programs developed in close working relationships with OER. 
Major Program Summary

(Fiscal Year Operating and Capital Budget Authority, \$M)

\begin{tabular}{lrrrr}
\hline Major Program/Office & 1992 & 1993 & 1994 & 1995 \\
\hline Office of Energy Research & 181.1 & 157.2 & 166.5 & 185.7 \\
Energy Efficiency \& Renewable Energy & 16.2 & 18.1 & 22.8 & 23.8 \\
Fossil Energy & 2.2 & 2.2 & 2.3 & 2.3 \\
Environmental Restoration \& Waste Mgt. & 14.7 & 10.4 & 20.7 & 18.8 \\
Environment, Safety, and Health & 1.8 & 2.6 & 2.6 & 2.7 \\
Other DOE & 27.2 & 20.1 & 25.3 & 23.5 \\
Work for Others & 46.3 & 51.2 & 68.5 & 59.3 \\
Total & 289.5 & 261.8 & 308.7 & 316.1 \\
\hline
\end{tabular}

\section{OFFICE OF ENERGY RESEARCH}

During the plan period, OER will continue to be the focus of fundamental science and engineering research activities at the Laboratory, implementing the initiatives described in Section 4 and growing in selected areas of the Basic Energy Sciences and Life Sciences. Many of these programs will be conducted in cooperation with industrial and academic research partnerships.

- For FY 1994 the Laboratory's contribution to national efforts in the Basic Energy Sciences (BES) includes scientific programs at the Advanced Light Source (ALS) and developing advanced user facilities to support scientists in chemistry, biology, materials research, physics, and other fields.

- In the context of an advancing national program in health and environmental research, LBL's human genome, structural biology, and cell and molecular biology programs will continue to provide the technical capabilities to improve the understanding of environmental and genetic control of diseases and normal functions. The biomedical program will improve diagnostic imaging systems and elucidate the metabolic basis of disease.

- High-energy physics research will continue to make major advances in detector and accelerator systems with a sophisticated new detector for the SSC and plans for a B-factory detector and collider. LBL contributes to the operation of current forefront facilities, including the DZero and Collider Detector Facility (CDF) at Fermilab.

- The 88-Inch Cyclotron with its Advanced Electron Cyclotron Resonance ion source provides the highest flux of heavy ions of any low-energy accelerator in the U.S. The Gammasphere detector, located at the Cyclotron, has started conducting new research in nuclear structure. The relativistic heavy-ion research program will be pursued with the STAR experiment at the Relativistic Heavy-lon Collider (RHIC) and with lead beams at CERN. The Sudbury Neutrino Observatory will be providing data to address the solar neutrino problem and the question of neutrino oscillations. 
This graph of the beam current in the storige ring of the Advanced light Source marks the major milestone when it erce'eded the design specification of $400 \mathrm{~mA}$.
$[\mathrm{mA}]$

500

400

300

200

100

0

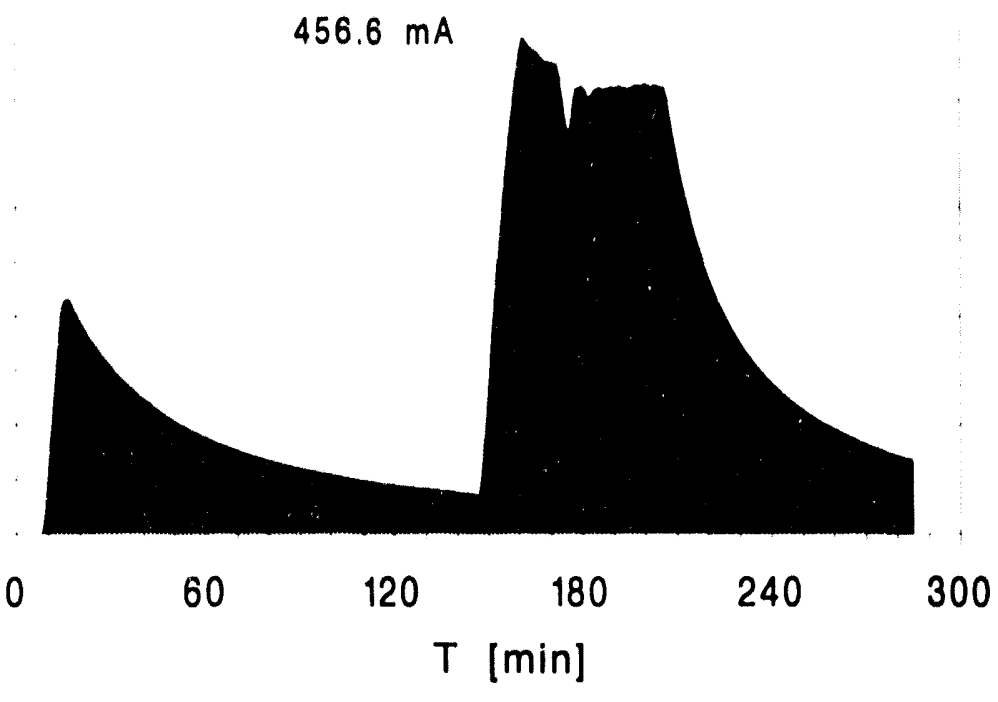

- In support of the national fusion research goals, LBL's programs in heavy-ion fusion accelerator research for inertial-confinement fusion and in neutral-beam development for magnetic-confinement fusion may expand significantly. These programs build on expertise in induction-linac systems and ion-source development.

- The Center for Advanced Materials (CAM) will continue to pursue Laboratory goals for conducting longer-term research responsive to industrial needs and the transfer of the results of the work to industry. Expanded program activity in CAM is anticipated in thin-film

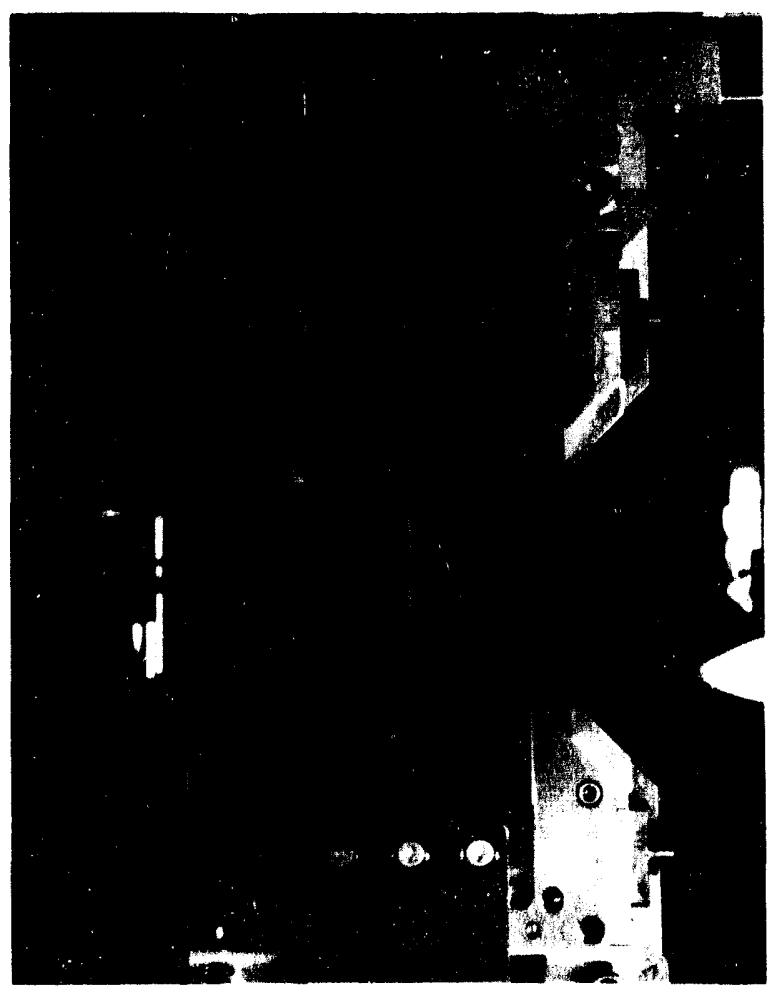
research, studies of wear and mechanical properties of surfaces, atomic scale synthesis of materials, and enzymatic synthesis of materials.

- The National Center for Electron Microscopy (NCEM) will continue to provide forefront research facilities for metallurgy, ceramics, and other materials research. Advanced microscopes for quantitative atomic resolution and analytical studies and studies of magnetic materials are proposed to maintain the nation's research leadership (see Section 4).

LBL will continue providing OER programs with the most advanced engineering research for instrumentation, such as magnet technology devices and advanced control systems. Program activity for OER is summarized in the table below.

Simulating an electron beam, a strobe light shines through the two arrays of magnets in an ALS undulator. The gap between the upper and lower rows of magnets can be changed to alter the wavelength of the electron motion, and thereby the photons emitted through synchrotron radiation. 
Office of Energy Research Funding Summary

(Fiscal Year Operating and Capital Budget Authority \$M)

\begin{tabular}{lrrrr}
\hline Major Program (BR Code) & 1992 & 1993 & 1994 & 1995 \\
\hline Basic Energy Sciences (KC) & 63.8 & 61.3 & 63.5 & 67.4 \\
Nuclear Physics (KB) & 48.2 & 35.4 & 37.2 & 35.4 \\
High-Energy Physics (KA) & 22.7 & 21.8 & 21.8 & 22.7 \\
Superconducting Super Collider (KS) & 0.4 & 0.0 & 0.0 & 0.0 \\
Biological \& Environmental Research (KP) & 21.3 & 21.7 & 23.9 & 38.2 \\
Fusion Energy (AT) & 9.1 & 8.6 & 7.9 & 8.2 \\
University and Science Education (KT) & 2.5 & 2.5 & 2.9 & 3.0 \\
General Purpose Facilities (KG) & 11.0 & 4.1 & 5.3 & 6.7 \\
Laboratory Technology Transfer (KU) & 2.1 & 1.8 & 4.0 & 4.0 \\
Total & 181.1 & 157.2 & 166.5 & 185.7 \\
Percent of LBL Total & 62.6 & 60.0 & 53.9 & 58.7 \\
\hline
\end{tabular}

\section{Basic Energy Sciences}

LBL is one of the world's leading centers of research in materials science and the chemistry and physics of materials that are important to both the production and efficient use of energy. In addition, outstanding programs exist in advanced energy projects, in engineering and geosciences, in biological energy research, and in applied mathematics. Several of these programs are expected to increase, as indicated below.

\section{Materials Sciences}

BES programs in Materials Sciences will continue to emphasize new and forefront research projects for the synthesis, processing, and characterization of advanced materials. Expanded areas include design and construction of synchrotron-radiation instrumentation and its application to important programs in materials sciences, and advanced materials synthesis and fabrication technologies, especially at the atomic scale. Leading programs continue in $\mathrm{x}$-ray optics, electron microscopy, solid-state physics, surface sciences, catalysis, polymers, biomolecular materials, metallurgy, electrochemical materials, electronic materials including super- and semi-conductors, ceramics, and materials chemistry. The ALS, a third-generation synchrotron-radiation facility, will produce the world's brightest beams of ultraviolet and soft $x$-ray radiation. The commissioning of the injection system and storage ring has been completed. The ALS, CAM, Center for XRay Optics (CXRO), and NCEM are organized interdisciplinary research centers that bring DOE resources to bear on scientific challenges of national importance.

In support of the ALS, the Laboratory is conducting research on storagering physics and engineering, ultrahigh-vacuum technology, instrumentation and feedback systems, insertion devices, beamline optical systems, and magnet systems. At CXRO, research is conducted on advanced opticalsystem components for the utilization of high-brightness photon beams. 
A conversien process has been developed to obtion polycrystalline silicon combide ceramic tibers. Thermally stable ceramic fibers have value as the reinfercing phase in high-performance composite materials for applicallions in energy and related industrial technologies such as gas turbine power generaltors, healt recovery equipment, waste incineration systems, burmers and combustors.
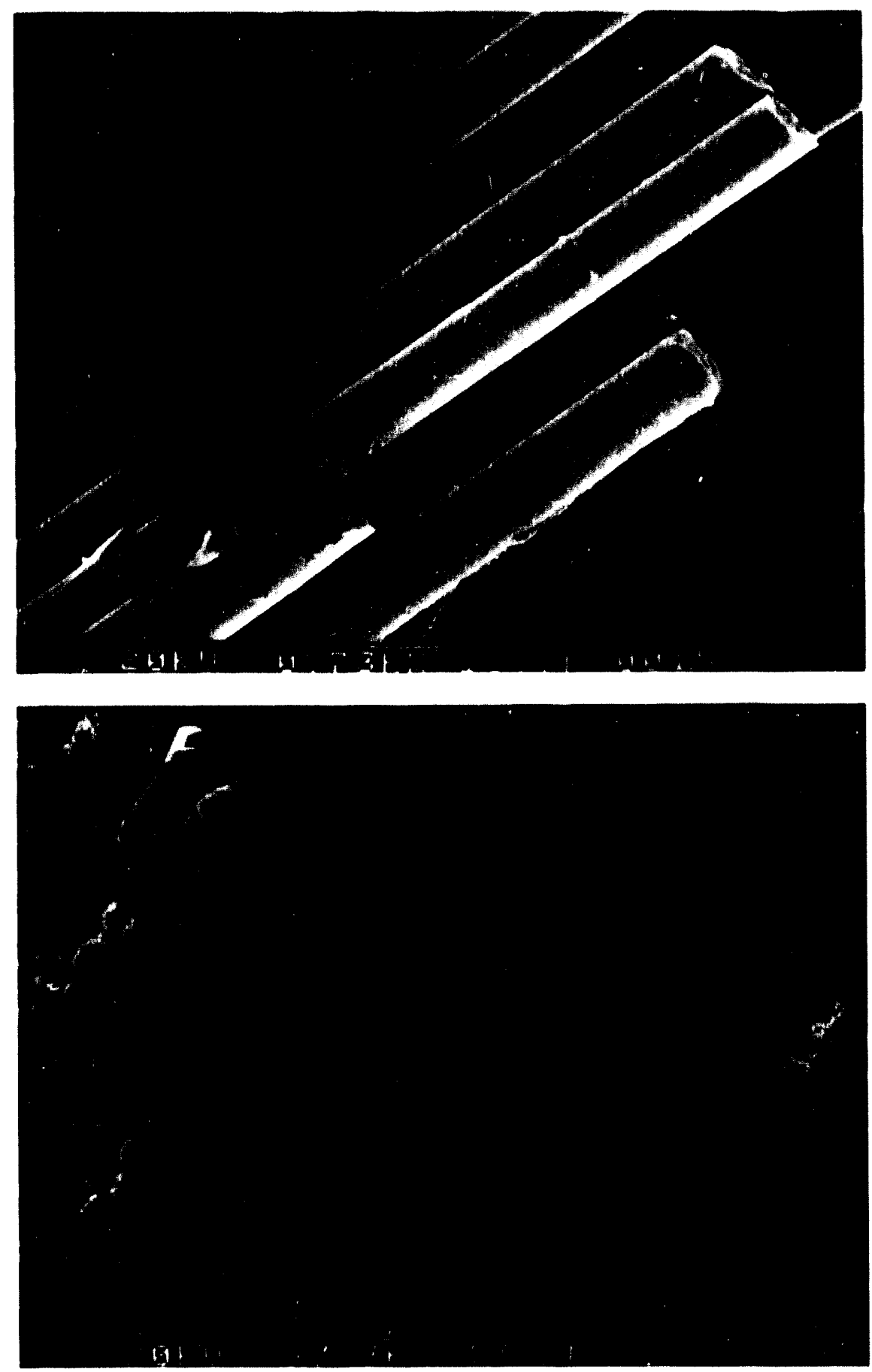

CAM will continue major research efforts selected because of their potential impact on U.S. industrial strengtin. The research focus evolves with new discoveries and with the changing needs of U.S. industry for fundamental research to underlie its development and commercialization activities.

- The Surface Science and Catalysis Program focuses on studies of advanced catalysts for clean fuels, pollutant reduction and methanol synthesis, and the atomic scale surface structure and chemical and mechanical properties of solids and adsorbed monolayers. Advanced surface instrumentation includes nonlinear optical techniques, scanning tunneling and atomic force microscopies, and advanced Raman spectroscopy. 
- The Electronic Materials Program focuses on theoretical and experimental studies of basic materials problems pertinent to the develop. ment of advanced electronic and optical materials. Semiconductor thin-film crystal growth and characterization, and comprehensive investigations of structural properties of heterointerfaces are pursued.

- The Polymers and Composites Program pursues polymer synthesis and studies of the relationships between polymer processing and micro. structure, focusing on anisotropic materials, and surface interactions between polymer licquids and metals.

- The High-Performance Metals Program addresses the mechanisms of structural failure, including fatigue and fracture in metals and interme. tallics, and is concerned with the theoretical and experimental studies of the properties and development of metal alloys, formable steels, advanced intermetallics, and materials for high-field superconducting magnets.

- The Ceramics Science Program supports research on the development of predictive, quantitative theories of densification and microstructure development, the application of these theories to produce and evaluate advanced structural ceramics with improved high-temperature performance, and new understanding of fatigue and other failure mechanisms in ceramics.

- The Biomolecular Materials, Enzymatic Synthesis Program explores the application of recent advances in biochemistry, molecular biology, and bioorganic chemistry to the synthesis of novel materials. Emphasis is on the use of natural, genetically engineered and "created" enzymes, and self-assembling thin films to control surface properties and form the basis of sensors.

- The High-Tc Superconductivity Program focuses on basic science, including theory, synthesis, and characterization of new materials; and on thin films and their applications, including devices such as SQUIDs and bolometers.

CXRO continued its two complementary roles: developing state of the art $x$-ray optics and demonstrating their utility for new science and technol. ogy in the extreme ultraviolet (EUV) and soft x-ray (SXR) regions of the electromagnetic spectrum. High resolution $x$-ray microscopy continues to be prominent among our activities, with applications directed at both the physical and life sciences. For example, our SXR microscopy based on fresnel zone-plate lenses has produced images of features as small as $300 \AA$. These microscopes are widely used for biological microscopy and surface science at synchrotron radiation laboratories around the world. Other efforts include an SXR photoelectron microscopy beamline, based on multilayer-coated reflective optics, for material and surface science at the University of Wisconsin Synchrotron Radiation Center. In the hard x-ray regime our microprobe has achieved $2-\mu \mathrm{m}$ spatial resolution at the National Synchrotron Light Source (NSLS) and has been used in a large number of applications. New efforts include projects in SXR projection lithography (Section 4) and in development of a national resource center for biological microscopy, spectroscopy, and crystallography at the AI.S.

NCEM, a national user facility, is supported by the DOE Metallurgy and Ceramics Program but contributes substantially to research in other fields such as biology and geology. The heart of NCEM consists of two micro- 

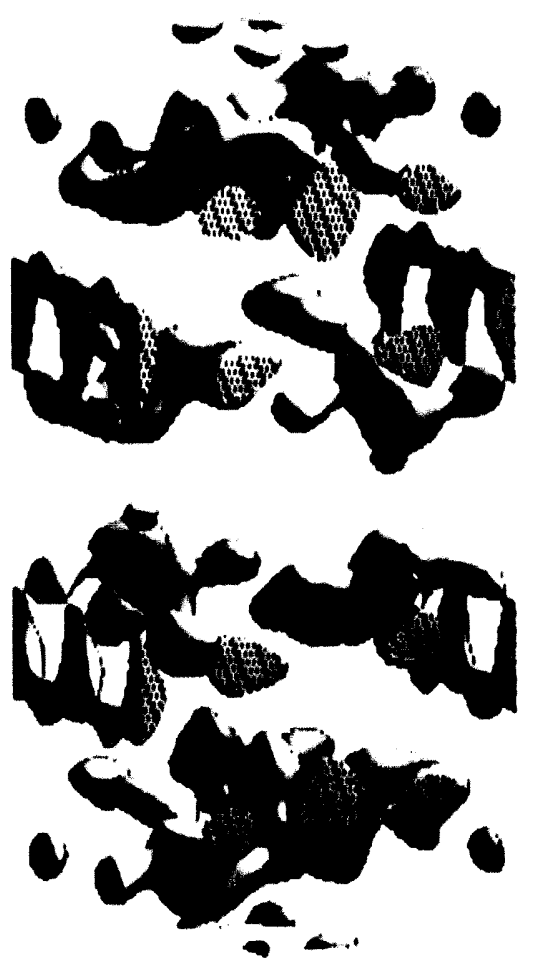

The tirst atemic resolution threedimensional Coulomb postential mape at matterials have been preduced at the Natrumal Center tor Electrom Alicroscopy using dilla irom the high-angle-thlt teature of its Alomic Resolution stic roscopes. To produce thes i-lo imager, high. resolutum ! 1.6 A 2.1 ) manges of the

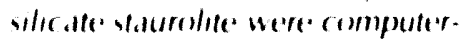

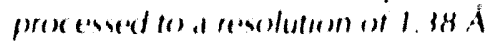
and combined tes bow the b-D coutemb pote itial as the cryatel and allow visualizatlen on all the atoms that lie in any geven platre through the material. scopes: (1) the High-Voltage Llectron Microscope, the most powerful microscope of its kind in the U.S., and (2) lhe Aicomic Resolution Micro. scope, with a resolution of $1.5 \AA$, currently the highest resolution in the world. Enhancement of the Center through the design and acquisition of new state-of the art microscopes is being proposed to maintain U.S. leadership in electron microscopy (see Section 4).

Research at NCEM is carried out on a wide range of materials, including studies of high-lemperature superconducting materials, structural materials, magnetic materials, ceramics, and amorphous silicon semiconductor materials. Research on the structure and properties of Iransformation interfaces has the goal of determining the atomic configuration at structural boundaries and the relationship between structure and properties at the interface.

LBL participates in the DOE Center of Excellence for the Synthesis and Processing of Advanced Materials. The Director of the Materials Sciences Division is the national coordinator for the focus area on Atomically Structured Materials, one of five focus areas in the center. This area of research has attracted the interest of a number of federal agencies and LBL is hopeful that its research program can, with appropriate collaborative infrastructure and operating support, make a major contribution to the field. Materials Sciences Division investigators also lead projects in the other four focus areas of Complex Polymer Systems, Advanced Ceramics and Ceramic Thin Films, Nanophase Materials, and Emerging Materials and Processes.

In addition to the research conducted in focused centers described above, LBL conducts materials research in further support of DOE's Metallurgy and Ceramics Program. Solid-State Physics Program, and Materials Chemistry Program, as described below.

in Metallurgy, LBL projects involve the development, characterization, and understanding of advanced alloys and intermetallics for energy needs. Specific research projects focus on investigations and structural observations of the ordering phenomena in alloys, with emphasis on long-period ordered superstru tures in binary f.c.c. alloys. There are also sludies aimed at understanding the fundamental features that underlie the evolution of microstructures in solids by applying crystallographic techniques to the analysis of topology and defects in crystalline materials. Another component of the research seeks to achieve a detailed understanding of the role of interfaces in materials, using high resolution transmission electron microscopy. One project is refining a method of three-dimensional structure determination using the Alomic Resolution Microscope at NCEM. Research continues on the synthesis and characterization of atomically engineered thin films with novel magnetic, optical, and mechanical properties. Solidstate phase-transformation mechanisms are also examined with the goal of understanding the structural factors that govern phase changes and phase stability.

In Ceramics, the L.BL program is again focused on developing advanced materials, both monolithic and composite, with superior mechanical propertices. Several projects are centered on the processing of improved compositions, both by special coating techniques applied to reinforcement phases for composite materials, and by modification of grain-boundary phases in monolithic materials. Mechanical property evaluation is concerned primarily with the mechanisms of toughness, oxidation, and creep behavior and the performance of ceramics under cyclic fatigue conditions, with particular emphasis on elevated-temperature behavior. In addition, the 


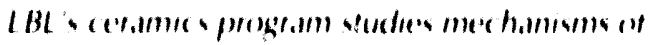

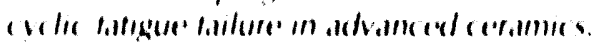

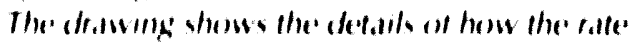

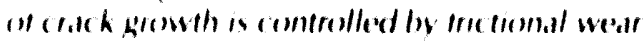

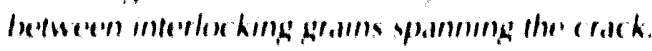

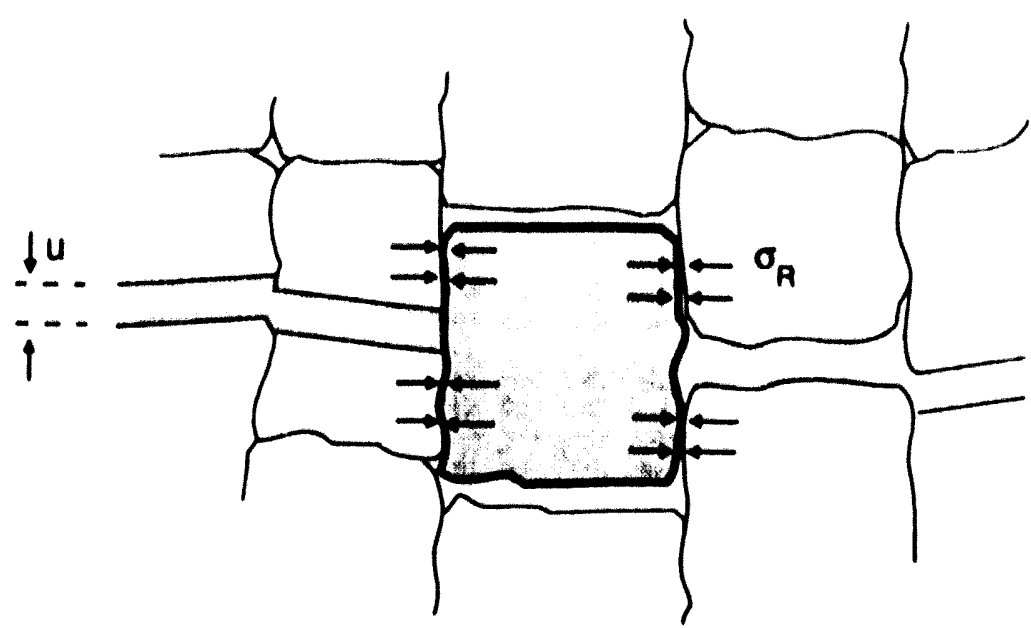

joining of ceramics to metals and other ceramics is being exarnined using novel techniques, such as transient liquid-phase bonding.

A new research program is planned to characterize the mechanical properties of advanced materials at extreme temperatures. An integral part of this program will be evaluating the deformation and fracture properties of advanced ceramics and intermetallics at temperatures up to $1600^{\circ} \mathrm{C}$ and above.

In Electronic Materials, LBL research focuses on an improved understanding of the materials science of artificially structured semiconductor and semiconductor-metal systems. Basic studies concentrate on the relation. ships between synthesis and processing conditions and the properties of semiconductor materials, as modified by the resulting structural and elec. tronic imperfections. Projects include the synthesis and study of heterointerfaces between highly dissimilar materials in order to understand mechanisms of interface formation and the defect structures resulting from lattice mismatch and differences in ionicity and electrochemical potential. The structure and stability of defects and interfaces are studied by advanced characlerization techniques. Atomic resolution microscopes at the National Center for Electron Microscopy and advanced $x$-ray techniques utilizing synchrotron radiation provide structural information. Optical spectroscopies ranging from the near UV to the far-infrared region of the electromagnetic spectrum, electron paramagnetic resonance spectroscopy and electrical transport measurements reveal complementary electronic properties. Theoretical and experimental work on the eifects of atomic scale diffusion and the differences between solid solubility limits of dopants and the maximum concentration of iree carriers is pursued. Novel types of processing methods inc luding annealing under large hydrostatic pressures and with funable synchrotron radiation, to increase the electric ally active fraction of dopants, are explored. Progress in this area is applic able to design of advanced photovoltaic energy conversion devices and of a large variety of sensors used in energy conversion processes.

Solid-State Physics research al L BL will continue with strong programs in both experimental and theoretical physics. One project focuses on furthering basic understanding of laser interaction with matter and applies 
modern optical techniques, including novel linear optical diffraction and infrared-visible sum-frequency generation, 10 moterials sciences research. Studies are also directed toward advancing the state-of-the-art in fermosecond laser pulse generation and measurement. Another research element involves investigation of the ultrafast responses of nanoscale materials 10 a variety of excitations and the development of new and stateof-the-art techniques in time resolved spectroscopy, femtosecond-time resolved spectroscopy, and the generation of ultrashort (100 fs) pulses. In superconducting devices, researchers apply new lechniques, including novel spectroscopy methods, for the investigation and development of superconductor structures, including superconducting quantum interference devices, magnetometers, and losephson junction amplifiers. Time-resolved spectroscopies in solids study the fundamental properties of semiconductors which have potential applic ations in electronic devices. The purpose of this work is to advance understanding of ultrafast relaxation processes of electrons excited either optically or electrically. In addition, the Solid-State Physics Program involves synthesis characterization and potential application of novel fullerene-based materials. A project on the quantum theory of materials has as its purpose the study of the structural, electronic, magnetic, and chemical properties of solid surfaces. Another project investigates the nonlinear phenomena in solid state systems and develops models that can explain and predict the observed behavior.

Working with the unique capabilities of LBL.'s new Advanced Light Source, projects will perform surface, interface, and nanostructure studies using synchrotron radiation and develop techniques of photoelectron diffraction and photoelectron holography as probes of near-surface atomic structures. One research project will utilize a new method with $x$-rays for the studies of the in situ interface at metal/solution interfaces. There is also research directed at understanding and unraveling the picometer-scale structure $(0.01 \AA$ ) of interfaces using synchrotron radiation at LBL's Ad. vanced light Source.

In Materials Chemistry, programs at L.BL. will continue to make strong contributions in interface synthesis and physical characterization through the development of new methods in atomic force microscope/scanning tunneling microscope, and photoelectron tunneling spectroscopy. Important new research to synthesize and characterize advanced materials with novel properties, particularly new classes of rare-earth transition metal phosphides that exhibit cooperative phenomena such as superconductivity is underway, along with studies on the low temperature properties of materials, with particular emphasis on superconductors. Another research clement is developing new concepts and techniques, such as optical pumping, in nuclear magnetic resonance and designing and fabricating the next-generation of nuclear magnetic resonance equipment.

One new initiative is examining the atomic-scale mechanical properties of surfaces and lubric ants: adhesion, friction and wear. A goal is to understand the role of surface films of lubricants in modifying these tribologic al preperties. A cecond initiative foc uses on the synthesis and characterization of heterointerfaces compesed of highly dissimilar materials. The geal of the batter project is 10 understand the growth me hanisms of heterointerfaces and to investigate their atemic and electronic structure. This project also involves developing new fee hniques for their synthesis and characterization. 
The schemallic diagram shows the unimolecular dissociation of ketene (CH.C()). The ketene molecule is prepared in a vibrationally excited state by absorption of laser light. which is followed by a localization of energy in the C.C. bond. As more energy is pumped into the molecule. vibriflunal excitation at the transulion vate the sadelle point ot the ene gy surfaces becomes possible. Stepwise increases in

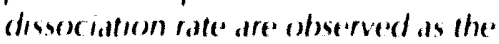
energy increases above the threenold fur each vibrational stale. This confirms the fundamental assumptions of statistical theorries for the rate of unimolecular reactions and demonstrates that ab initio calculations for transition stalles rall be reliable.

\section{Chemical Sciences}

DOE's (hemical Sciences Program supports focused research in soveral LBL divisions. Efiorts in the (hermical Sciences Division (CSD) emphasize chemical physics, dynamics and mechanisms of chemical reactions and combustion processes, calalysis, electron spectroscopy, atomic physics, photochemistry, theoretical chemistry, and chemistry of the actinide elements and their relationship to environmental issues. Programs in the Energy and Environment Division focus on advanced combustion and the mechanisms for minimizing emissions and improving fuel efficiency. In the Structural Biology Division, programs in photochemistry and the chemistry of electronically excited molecules are conducted. In the Nuclear Science Division, a study of the chemical properties of the heaviest elements is being conducted.

In CSD, the extensive chemical physics research includes several major programs. One has focused on a study of the spectroscopy and structures of reactive intermediates. Laser-induced fluorescence, multiphoton ionization, and negative-ion photodelachment have been used to stirlu reartive molecules such as free radicals and cluster ions that may $b_{x}$. inportant in combustion processes, reactive plasmas, and high-technology manufacturing processes. Techniques such as the use of crossed molecular beams are used for advanced and novel studies of the dynamics of important reactions with the goal of understanding elementary chemical reactions through single collision conditions or laser excitation. This research on reaction dynamics and combustion is expected to grow during the next several years.

The program in reactivity at surfaces and interfaces will involve molecular studies of interfacial phenomena using new techniques in laser spectroscopy and $x$-ray scaltering. The program is designed to gain an understanding of chemic al reactivity in key areas of energy science, including nearly all

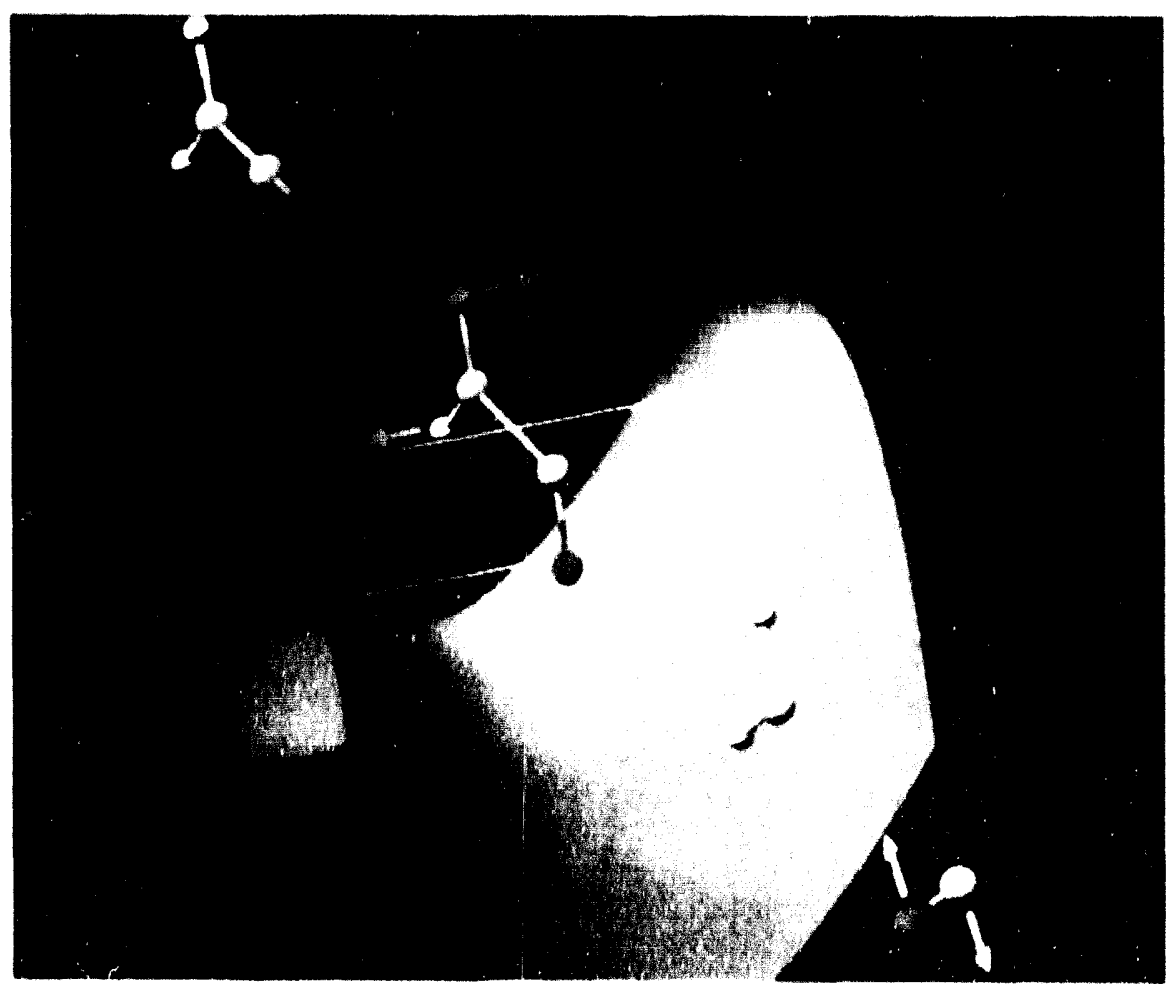


Catalytic reaction systems, solar-energy conversion fechnolegies, lightt. assisted chemical syntheses, electros hermic al-energy-comversion fechnolegies, and corrosion phenomena.

Chemical energy research at LBL has revealed new read tions between transition metals, such as heniun, and organic molecules that are important to the improvement of catalysis involved in coal-conversion processes. Contınuing program areas are focused on the fundamental chemistry of important environmental and fuel species, including aqueous and gaseous species of carbon and sulfur. Catalytic conversion of carbon monoxide and hydrogen to gaseous and liquid fuels is studied to develop more efficient catalysts for hydrocarbon production.

The research programs in theoretical chemistry have the goal of accurately predicting chemic al reaction dynamics, especially those that are too complicated to be solved experimentally. The program on photochemical and radiation sciences includes research into the photochemistry of materials in the stratosphere (with applications to the role of trace gases in the "greenhouse effect").

Research in the actinide chemistry program in CSD has two thrusts, the design and synthesis of sequestering agents for treatment of actinide poisoning and for possible application to spent reactor fuels, and the preparation and study of ne'w compounds incorporating actinides. A program to investigate the chemical properties of the heaviest elements $(Z$ of 102 to 106) at the furthest reaches of the periodic table is being undertaken in the Nuclear Science Division.

Research in the Energy and Environment Division includes theoretical and experimental programs on ignition, reactivity, turbulence, and energy transfer in combustion systems. Advanced approaches include studies of photodissociation, laser spectroscopy methods, and molecular-beam nass spectroscopy and the use of unimolecular kinetics for the theoretical study of high-temperature reactions important to combustion. Another area of research is laser-material interactions for chemical analysis.

Research at L.BL's Structural Biology Division is directed at a fundamen. tal understanding of the chemistry of electronic ally excited molecules, with altention on features that relate to the conversion of photon energy and the photo-assisted synthesis of high-value compounds. Projects focus on the manganese catalytic function in artificial photosynthesis, the photoinduced reduction of $\mathrm{CO}_{2}$ into organic products, and polyelectrolyte interfaces for increasing quantum efficiency in photosynthetic processes. Other work explores chemistry induced by red and near-infrared light, the most abundant form of energy available from the sun. Foc us is on redox reactions in solution that may lead to new concepts for chemical storage and conversion into electric ity of these long wavelength quanta, and on their use for controlled photoc hemic al synthesis in a solid matrix. A main new effort is direc ted at monitoring of elementary reaction steps in hese enviromments by time-resolved fourier-transform infrared spectroscopy.

\section{Engineering and Geosciences}

The ciecsciences Program at L.BI is strengthening its multidisciplinary (effort in) expand the scientific basis of many energy-related tec honologies, inc luding development of hydrof arbon and strategic -mineral resources, safe disposal of radieactive and loxic chemical wastes, and explestation of geothermal energy. Farth seiences researchers at LBL. are among the leading experts in the areas of subsurface imaging of the structure and dynamic sof 


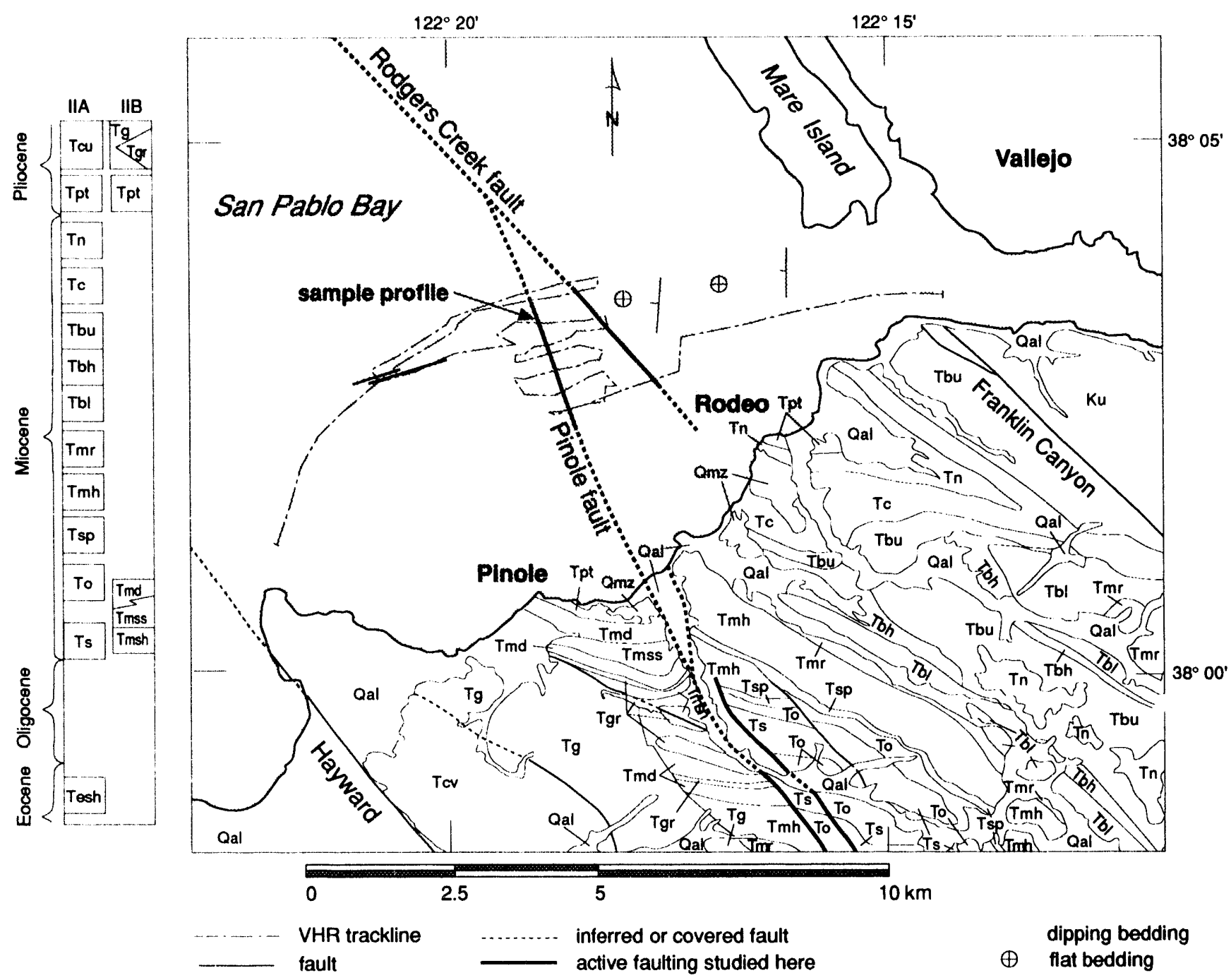

$\angle B L$ is a leader in technology development for high resolution subsurface imaging. For example, in fall of $1992 \mathrm{LBL}$ geologists recorded prototype very high resolution images of faulting in layered silts beneath the waters of San Pablo Bay. These studies provide the first evidence of activity of the Pinole fault, give the detailed geometry of faulting in the Pinole area, and indicate the earthquake recurrence intervals. the earth's crust, experimental investigation of the mechanisms by which lithospheric processes influence energy resources, and numerical modeling of geochemical and hydromechanical processes occurring in heterogeneous fractured rock formations.

Geohydrology research at LBL includes experimental studies of the physical behavior of fluid-saturated rock, numerical modeling of coupled processes in subsurface reservoirs, and investigation of the mechanisms associated with chemical transport and multiphase fracture-flow phenomena. New algorithms are being developed to greatly increase the efficiency of the numerical solution of flow and transport equations.

Geophysicists, supported by LBL's Geophysical Measurements Facility and the Center for Computational Seismology, are developing methodologies and instruments to define crustal structure over a range of scales, to monitor processes in subsurface reservoirs, and to track the movement of contaminant plumes in underground aquifers. Borehole seismic and electromagnetic source development coupled with real time signal processing are keys to improved high resolution imaging capabilities. At a laboratory scale, new approaches are employed to understand fracture processes and wave propagation in fractured media. Other geophysical research 
employs new computational codes to measure fracture properties in subsurface reservoirs and to map hydrofractures at well sites.

Geochemical studies focus on the thermodynamic properties of electrolytes, the generation and migration of petroleum compounds, and the interactions between minerals and groundwaters. Analytical capabilities of the Center for Isotope Geochemistry provide a powerful means of characterizing natural systems. This Center is an important element in many of the multidisciplinary geological and environmental investigations at LBL. It provides a focus, in collaboration with geophysicists and geologists, for continised study of crustal processes as part of the multiagency Continental Scientific Drilling Program.

\section{Energy Biosciences}

LBL's program continues to improve understanding of the unique features of photosynthetic organisms for collecting light energy and storing it as chemical energy. One project uses spectroscopic techniques to map the components and the kinetics of the light reactions. The genetics of the photosynthetic apparatus of single-cellrd organisms are studied to allow application of DNA-cloning techniques to elucidate photosynthetic mechanisms. The light regulation of gene-encoding components of the photosynthetic apparatus in plant protoplasts is also being investigated. The DOE Division of Energy Biosciences is also supporting research in LBL's CAM on the enzymatic synthesis of materials.

This schematic details a model, developed from electron paramagnetic resonance and $x$-ray absorption spectroscopy, for the understanding of the mechanism by which $\mathrm{H}_{2} \mathrm{O}$ is converted to $\mathrm{O}_{2}$ and reduced products in photosynthetic organisms. This process will be most valuable for designing artificial solarto-chemical energy conversion svstems.

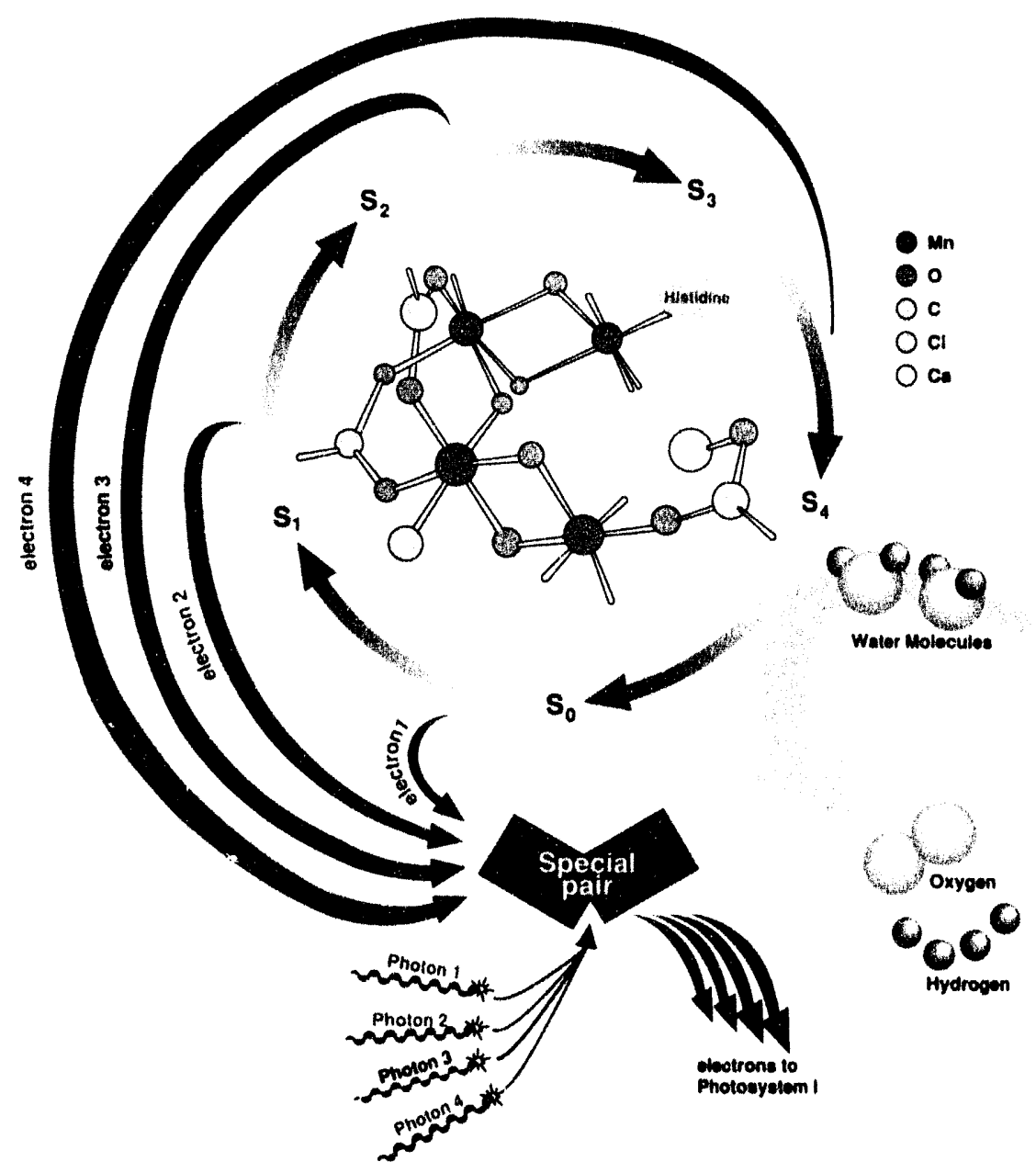




\section{Applied Mathematics and Computer Science}

The program in mathematics at LBL centers on the development of numerical and analytical methods and their application to the most challenging problems in physics and engineering. Investigations that were started within the LBL Mathematics Department have been at the frontiers of such topics as vortex methods, random choice techniques, high-resolution methods in gas dynamics, front-propagation techniques, and lattice and polymeric models in turbulence.

The three most active areas are particle and vortex methods, free-surface problems, and parallel processing. In the first area, recent successes include an analysis of superfluid turbulence by vortex methods, a model of the turbulent boundary layer, vortex renormalization, rapid implementation of particle methods, and numerical studies of engineering flows and of suspensions. In the second area, new algorithms based on Hamilton-Jacobi methodology, new surface-integral techniques, and other methods are being developed for freesurface problems; these techniques will be used for studying applications in differential geometry, combustion, flow in porous media, solidification, relativity, fluid instability, and capillarity. In the third area, software is being developed on a massively parallel processor for solving two-dimensional, viscous, incompressible fluid flow in arbitrary geometries and for scientific visualization. Other continuing areas of activity in mathematics include numerical linear algebra, finite-difference methods in fluid mechanics, Monte Carlo methods, and labor-partitioning schemes for multiprocessors.

The Scientific Data-Base Management Research Program will continue to investigate new data-management techniques suited to scientific and statistical applications. New requirements arise from the structure of some scientific data (e.g., sparse multidimensional tables, temporal data) and operation needs (e.g., transposition, aggregation, random sampling, proximity searches). Thus new efficient techniques for data-storage organization, new algorithms for data manipulation, and riew data-modeling methods to improve semantics of scientific data are being developed.

The Supercomputing Access Tools Program addresses the problems of scientific computing in distributed environments, with the goal of developing techniques that will partition the computational requirement optimally across distributed resources. The research on a software bus system will result in an ability to generate interoperable, and therefore reusable and replaceable, software. This will greatly enhance the computing environment available to energy-research scientists. Visualization and imaging tools compatible with this innovative architecture will be developed.

A new area of research, in collaboration with the Xerox Palo Alto Research Center, has been in multi-media conferencing. New protocols have been developed to support high quality audio and video transmission on the Energy Sciences Network and other parts of the Internet. In addition, work is being done to incorporate an interactive "whiteboard" or graphic display window in which network users can make real-time modifications.

\section{Nuclear Physics}

Nuclear physics research at LBL will continue to focus on the experimental and theoretical investigation of the structure and properties of nuclei, emphasizing studies of nuclei under extreme conditions (of temperature, isospin, angular momentum, and density, for example). The comprehensive research program and the unique facilities and instrumentation available at LBL are summarized below. 


\section{Relativistic Heavy-Ion Physics}

The main focus of the future high energy heavy ion research program at LBL is the Relativistic Heavy lon Collider (RHIC), which is currently scheduled to be completed at Brookhaven National Laboratory in 1997. For the nearer term, the various activities of LBL at the CERN Laboratory in Geneva, Switzerland have finished data taking with ${ }^{32} \mathrm{~S}$ beams and have now consolidated into one experiment, $\mathrm{NA49}$, for the $\mathrm{Pb}$ beams program in 1994. In the short term, the extensive local activities at the Bevalac have finished data taking, because of the shutdown of the Bevalac, and are now in the analysis stage.

The goal of many of the Bevalac experiments has been to compress nuclear matter sufficiently to study its equation of state, or how its density responds to the pressure built up in the collision. The higher energy program at CERN is attempting to compress nuclei sufficiently to produce a transition of the nucleons in the nuclei to a plasma of free quarks and gluons. At the still higher energies expected at RHIC the density of the energy of the produced particles will be so high that a momentary state of the quark-gluon plasma is expected. It is believed that the quark-gluon plasma existed soon after the Big Bang at the creation of the universe and may exist now in the cores of neutron stars.

In the RHIC Program, the STAR (Solenoidal Tracker At RHIC) Collaboration now has 210 scientists and engineers from 28 institutions. The spokesperson, project director, and thirty of the scientists are from LBL. STAR is an experiment to study particle prod:uction and high transverse momentum jet production at midrapidity to identify the phase transition from normal nuclear matter to quark matter. The experiment will contain a Time Projection Chamber (TPC), a three-dimensional imaging device, located inside a superconducting solenoidal magnet for tracking, momentum analysis and particle identification. STAR was approved as a project in early 1993 and detector design and construction are under way (see Section 4 for further details).

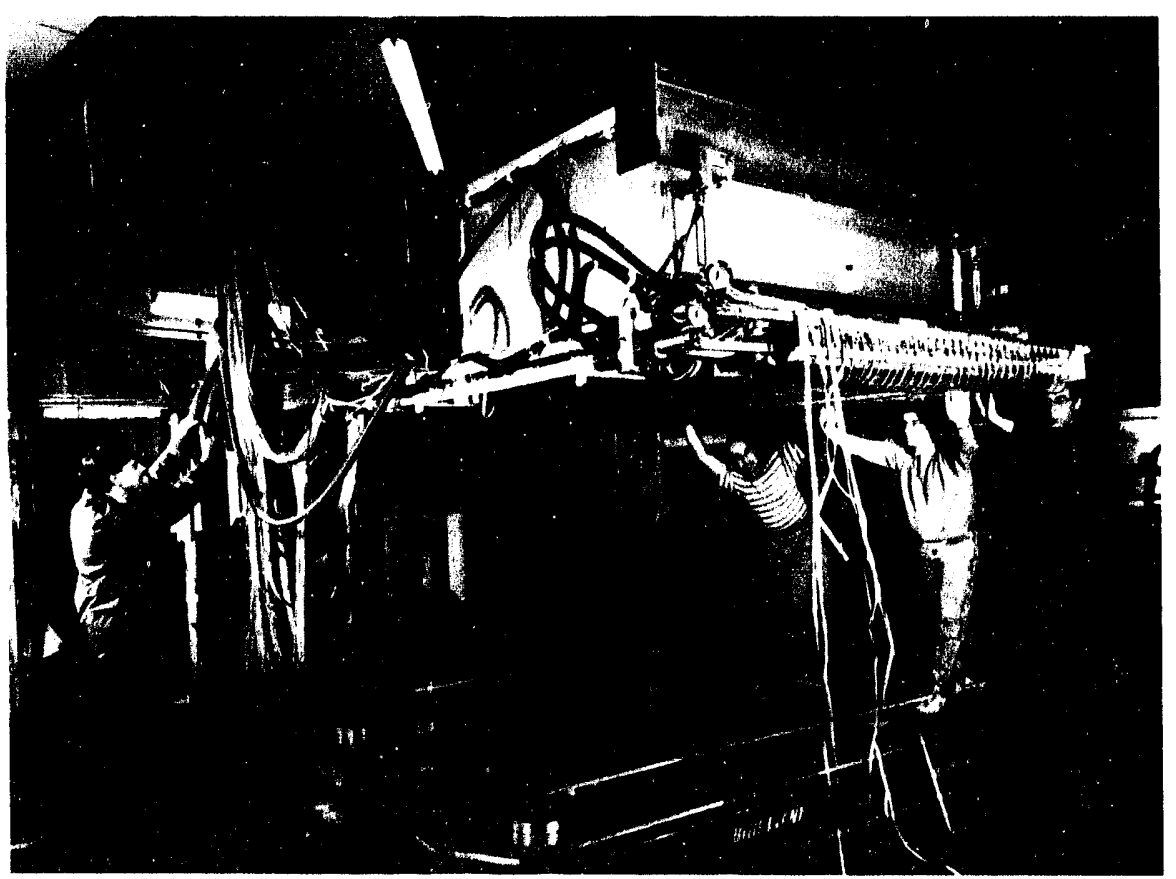

The Equation-Of-State Time Projection Chamber is being rolled out of the Bevalac experimental area for servicing. A successful series of experiments was performed with this detector prior to the 1993 closure of the Bevalac. In addition to the scientific contributions, technical experience gained with this detector will contribute to the design of the Time Projection Chamber for the STAR Detector at RHIC. 
At the CERN Super Proton Synchrotron (SPS) the emphasis of the research program is to explore the possibilities of producing a phase transition from hadronic matter to a quark-gluon plasma in central collisions of heavy ions at these energies. LBL effected a consolidation so that at the end of the recently completed ${ }^{32} \mathrm{~S}$-induced reaction program it was participating in only one CERN experiment: NA35. LBL built and installed 6000 additional channels of modern electronics for the NA35 TPC. This TPC was the first such detector to operate in a magnetic-field-free region with pad readout only. This was a step on the way to a much larger contribution from LBL of integrated electronics for the main TPC of the NA49 Pb-beams experiment in 1994. NA49 will consist of a combination of TPCs, dipole magnets and a time-of-flight wall that should allow enough coverage of the particles from the reaction to do event-by-event physics for the first time at a CERN experiment.

Concluding the Bevalac Program during the past year, there were three programs utilizing unique experimental equipment and beams to study the properties of nuclear matter that have accumulated copious data and are now in the analysis stage. First, the EOS time projection chamber (TPC) pioneered the technique of tracking by pad-readout only in a magnetic field and the use of high-density electronics using custom designed integrated circuits. EOS expanded on existing $4 \pi$ measurements and has obtained new data for complete event analysis of the central collisions of the heaviest nuclei. Secondly, the dilepton spectrometer (DLS) measured dilepton production in $\mathrm{Ca}+\mathrm{Ca}, \alpha+\mathrm{Ca}$, and $\mathrm{C}+\mathrm{C}$ reactions at several energies. The DLS collaboration finished a systematic study of the elementary $p-p$ and $p-n$ interactions at several energies. Finally, the production of radioactive beams and measurements of their ground-state properties were pioneered at the Bevalac in collaboration with several groups from Japan. Initial work involved the measurement of the radii of light neutron-rich nuclei, culminating in the observation of the "neutron halo" associated with ${ }^{11} \mathrm{Li}$. Work in this area continued to concentrate on measurements of the correlations between nuclear fragments from these exotic nuclei and played a worldwide leadership role in defining new directions for this line of research.

\section{Low Energy Nuclear Physics}

The broad and diversified low energy research program has five major components: nuclear structure, heavy element nuclear and radiochemistry, heavy-ion reaction studies, nuclei far from stability, and nuclear astrophysics/weak interactions. Some of these programs are carried out exclusively at the 88-Inch Cyclotron, others have portions at other accelerators, and others are non-accelerator based experiments. LBL scientists are playing important roles in the construction of two major new facilities: Gammasphere and the Sudbury Neutrino Observatory (SNO). LBL scientists are also participating in planning for the IsoSpin Laboratory, a proposed major new national initiative.

The research in nuclear structure is currently focused on the structure of the nucleus at very high angular momentum (high spin). The nuclear structure group has continued its strong physics output with numerous publications on the structure of superdeformed nuclei and the new oblate bands in the mass 190 region. At the same time the group is heavily involved in the construction of Gammasphere, the major initiative of the nuclear structure community. 
Photo of the Garmmasphere with 30 detectors installed at the 88 -Inch Cyclotron. Following a commissioning period, the Early Implementation Program has completed several successtul runs, detecting previously unobserved transitions. Completion of Gammasphere (with 110 detectors) is scheduled for late 1995.

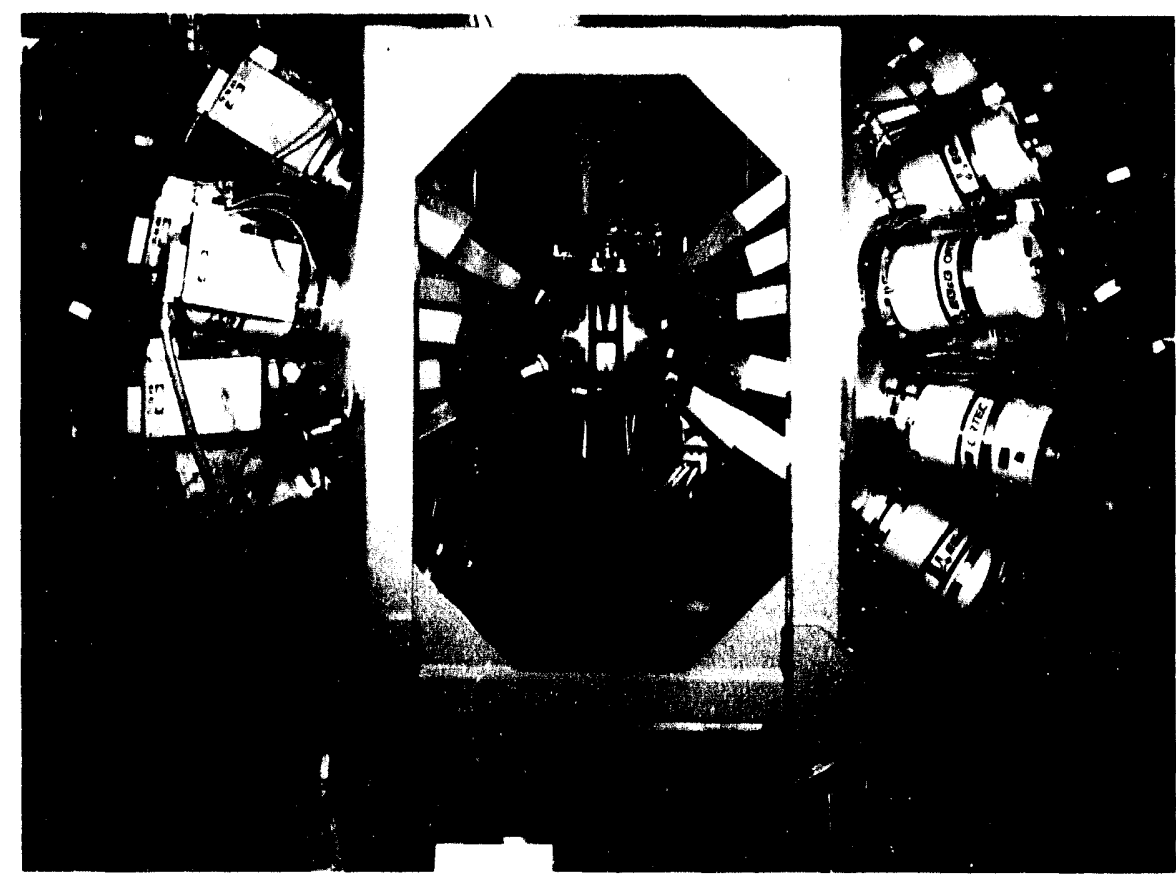

Gammasphere is under construction at LBL and will operate initially at the 88-Inch Cyclotron. Construction began in 1991 following the Baseline Review. Gammasphere consists of an array of 110 large Compton-suppressed Ge detectors that will have an efficiency for detecting 1-MeV gamma rays six times larger than any array existing prior to 1992, when Eurogam and GASP began operation. It will be possible to use five-fold coincidence events with Gammasphere, and these events will provide a resolving power that is around 100 times higher than that obtained from any of the arrays in use prior to 1992 and about 10 times higher than Eurogam and GASP. A broad range of nuclear physics can be addressed with such an instrument. Some examples include nuclear shapes (superdeformation, coexistence), warm nuclei (onset of chaos, giant resonance), symmetries in nuclei (Coulomb excitation), new regions of nuclei (secondary beams), and astrophysics (proton and alpha-particle capture cross sections).

Early implementation of Gammasphere began in March 1993 with 21 detector systems (soon to be 30 ) and five very successful commissioning runs were completed by early May; first phase operation will begin a year later with about 50 detectors, with completion of the facility in October 1995. (In an independent but parallel effort, an improvement in the performance of Gammasphere detectors is under study.) After it is fully operational, Gammasphere research is expected to occupy about one-half of the 88 -Inch Cyclotron's total beam time. Gammasphere will be operated by LBL's Nuclear Science Division as a national user facility, with active participation by groups at ANL, ORNL and about 20 other U.S. national laboratory and university research groups that joined in the proposal.

In the field of heavy element nuclear and radiochemistry, several factors have combined to make the 88-Inch Cyclotron facility one of the leading laboratories in the world for the production and study of transuranic nuclei. One factor is the availability of intense (microampere) beams of ions from ${ }^{1} \mathrm{H}$ through ${ }^{48} \mathrm{Ca}$. Another is the technology developed at LBL and LLNL for 
producing, handling, and irradiating radioactive targets of isotopes from uranium through einsteinium. This program will continue to use the Cyclotron to produce and characterize new elements and isotopes, to study nuclear reaction mechanisms, and to train students in modern nuclear and radiochemical techniques.

With the closure of the Bevalac, much of the heavy-ion multifragmentation program has been transferred to the $\mathrm{K} 1200$ Cyclotron at Michigan State University. A parallel program is maintained at the 88-Inch Cyclotron to determine the emission barriers for complex fragment emission from medium mass nuclei. More recently, a collaboration has been established to build a $4 \pi$ charged particle detector (MicroBall) that will fit inside the Gammasphere. The combination of the MicroBall and Gammasphere will be a very powerful tool for spectroscopic and reaction studies at the Cyclotron. In particular, studies of angular momentum transfer and alignment in deep-inelastic reactions with discrete $\gamma$ rays that, in the past, were hindered by poor statistics, will now become feasible.

A program in nuclei far-from-stability characterizing the decay of light proton-rich nuclides is carried out at the 88-Inch Cyclotron. There is also an ongoing program (carried out at several accelerators) measuring the spontaneous emission of heavy clusters from actinide nuclei. Work previously carried out at the Bevalac measuring the magnetic moments and nuclear polarization of radioactive nuclei will continue at the 88-Inch Cyclotron.

LBL scientists are participating in a national initiative, the IsoSpin Laboratory, which would provide intense beams of radioactive ions and open up new areas of research. LB! is represented on the IsoSpin Laboratory national steering committee, is conducting R\&D to answer key design questions, and is exploring a preliminary proposal to build such a facility. Currently, a high intensity target test stand is being constructed to study target cooling problems associated with intense proton beams from the proposed IsoSpin Laboratory (see Section 4 for further details).

In collaboration with eleven other institutions from Canada, the U.S., and the United Kingdom, LBL is participating in the Sudbury Neutrino Observatory (SNO), an experiment to detect neutrinos from the sun and from supernovae using a large $\mathrm{D}_{2} \mathrm{O}$ detector. $\mathrm{SNO}$ is designed to address the solar neutrino problem, the question of neutrino oscillations and neutrino production in stellar collapse. The LBL nuclear astrophysics group is playing a major role and has responsibility for the design and construction of the support structure to hold the some 10,000 photomultiplier tubes required. Installation of the support structure in Sudbury will begin in the fall of 1993 and first physics results are expected by the end of 1995 .

The new weak interactions group has started a program in laser trapping. The discovery that certain neutral atoms can be easily cooled and manipulated with laser light provides nuclear physicists the exciting opportunity to study nuclear beta decay with unprecedented precision. The initial goal is to apply the new techniques to radioactive ${ }^{21} \mathrm{Na}$. The beta-decay asymmetry of ${ }^{21} \mathrm{Na}$ will be measured for the purpose of studying the structure of the charge changing weak currents and to test the hypothesis that the weak interaction should be represented by a purely left-handed theory at the most fundamental level. A laser laboratory has been set up and efforts are underway to trap radioactive ${ }^{21} \mathrm{Na}$ produced on-line at the 88 -Inch Cyclotron. A ${ }^{21} \mathrm{Na}$ beam has been successfully focused by transverse cooling with a dye laser; this is the first time radioactive atoms have been cooled with laser light. 
The 18-meter (60)-ft) geodesic sphere hangs from a crane during the ribbon-cutting ceremony held to acknowledge the completion of the first integral component of the Sudbury Neutrino Oloservaltory (SNO). The sphere will hold photomultiplier detectors surrounding an acrylic tank full of heavy water, which will be uniquely sensitive to detection of solar neutrinos.

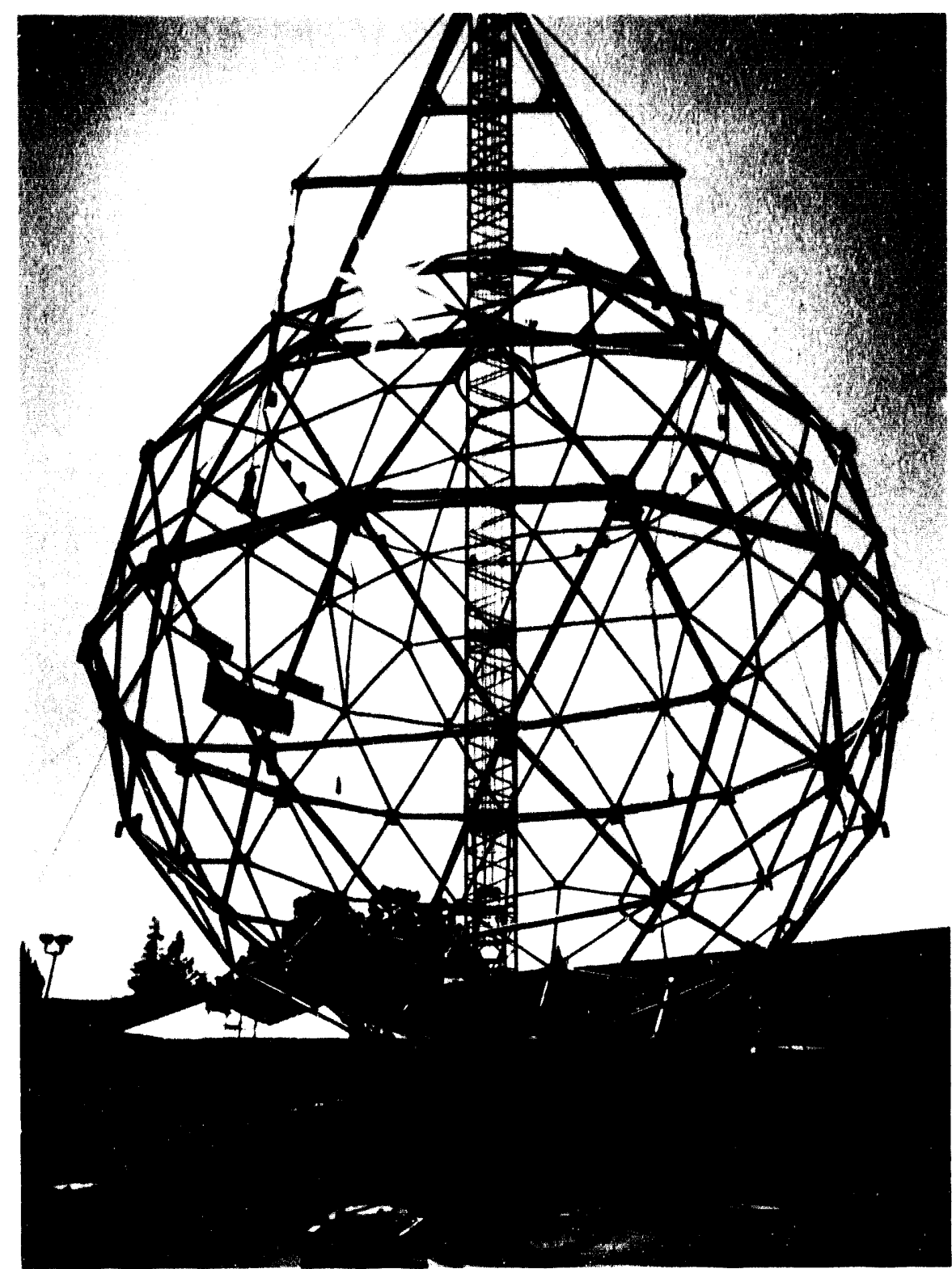

A few years ago, the LBL nuclear astrophysics group demonstrated that the beta spectrum from a ${ }^{14} \mathrm{C}$-doped germanium detector showed a distortion, which is consistent with a $17-\mathrm{keV}$ neutrino that is emitted with a probability of $1.25 \%$. Their recent very high statistics measurement of the inner bremsstrahlung (IB) spectrum of ${ }^{55}$ Fe shows no evidence for this distortion. This is strong evidence against the existence of a $17-\mathrm{keV}$ neutrino. This negative result was confirmed recently in an elegant measurement of the beta decay spectrum of ${ }^{15} \mathrm{~S}$ carried out by the weak interactions group. Using a high field solenoid spectrometer with no collimators, they were able to set very stringent limits on the emission of a $17-\mathrm{keV}$ neutrino.

\section{Nuclear Theory}

The goal of the nuclear theory program at LBL is to develop the theory and methods necessary for the analysis and interpretation of experiments involving nuclei and for the prediction of as yet unobserved phenomena. 
These include nuclear reactions at low to ultrarelativistic energies, and lepton-nucleus and hadron-nucleus reactions. In addition, the program aims at adding breadth to the Nuclear Science Division's overall nuclear research program by concentrated efforts also in nuclear astrophysics, macroscopic nuclear models, QCD and hadrodynamic theories of ultradense matter and phase transitions, and an interdisciplinary program in the order-to-chaos transition. New programs are being planned in preequilibrium parton dynamics and supernova simulations. A lively visitor program stimulates and further supplements these areas of theoretical research.

\section{Nuclear Data Evaluation}

The Isotopes Project of the Nuclear Science Division will continue to provide evaluated nuclear structure and decay data for the world nuclear physics community. It coordinates its efforts with the U.S. Nuclear Data Network and the IAEA International Nuclear Data Committee. In addition to its mass-chain evaluation activities, the group produces comprehensive: publications for both basic and applied users. The group is currentiy preparing the 8th edition of the Table of Isotopes, in both electronic and hard-copy versions. The group is exploring the feasibility of redirecting the conventional approach of mass chain evaluation to specialized horizontal evaluations of high spin and decay data. The group has an expanding role in the Network, particularly for electronic access and publication of the extensive data files; a modern database management system is being developed to facilitate this work.

\section{Accelerator Improvements}

Two improvement projects being carried out this year will optimize the 88-Inch Cyclotron's performance with regard to heavy ions and will directly benefit the Gammasphere experimental program now getting underway. Improvements in the cyclotron vacuum system will increase the intensity of heavy ions above mass 100 and a beam pulse reduction system will improve beam timing. Planned future improvements include modernization of the radiation safety interlock system and an upgrade of the AECR ion source so that it can operate at higher magnetic fields and frequency. The AECR upgrade will further increase the cyclotron's performance for the heavy ion beams $(A>100)$ and open up new research opportunities in the Gammasphere and complex fragmentation programs.

Several communities of users in the physical, chemical, and biological sciences and in materials and nuclear engineering have expressed their need for a pulsed, megawatt-class neutron source that is based on an accelerator and a production target. Complementing existing and proposed reactorbased sources, this "spallation" approach reduces the cost, simplifies the environment, health, and safety issues, and, most important, enables an additional class of experiments that requires pulses rather than a continuous flux. The DOE Office of Basic Energy Sciences has asked LBL to host a Spallation Neutron Source Design Study to examine the users' needs and available technology concepts and to produce a conceptual design. LBL offers the accelerator expertise necessary to contribute to and manage the design effort, and will not be in the competition for the neutron facility itself. 


\section{High-Energy Physics}

In high-energy physics, the Laboratory continues its strong and diverse program of experimental and theoretical research, including the development and operation of innovative detectors and research on advanced accelerator components and concepts. LBL is actively participating in the national effort to design future facilities - the SSC detector and accelerator components and an asymmetric B factory at the Positron Electron Project (PEP).

\section{Experimental Programs}

The Laboratory's experimental programs in high-energy physics focus on the properties of quarks and leptons, the basic constituents of matter. Their interactions are mediated through the gauge bosons, namely, massless photons and gluons and massive $W$ and $Z$ particles. Efforts at studying these particles emphasize the development of sophisticated detectors and their operation at colliding-beam facilities. Major experiments are in progress or in active preparation at SLAC and Fermilab.

A major achievement has been the very successful exploitation of the Silicon Vertex Detector (SVX) at the Collider Detector Facility at Fermilab. The $S V X$ project required innovative $I C$ design and read-out electronics, led by $L B L$ scientists and engineers, to measure tracks of charged particles with very high spatial resolution. The SVX improved vertex resolution by a factor of 25, enabling detailed studies of $B$ mesons including the lifetimes of the charged and neutral $B$ mesons and evidence for the $B$, meson.

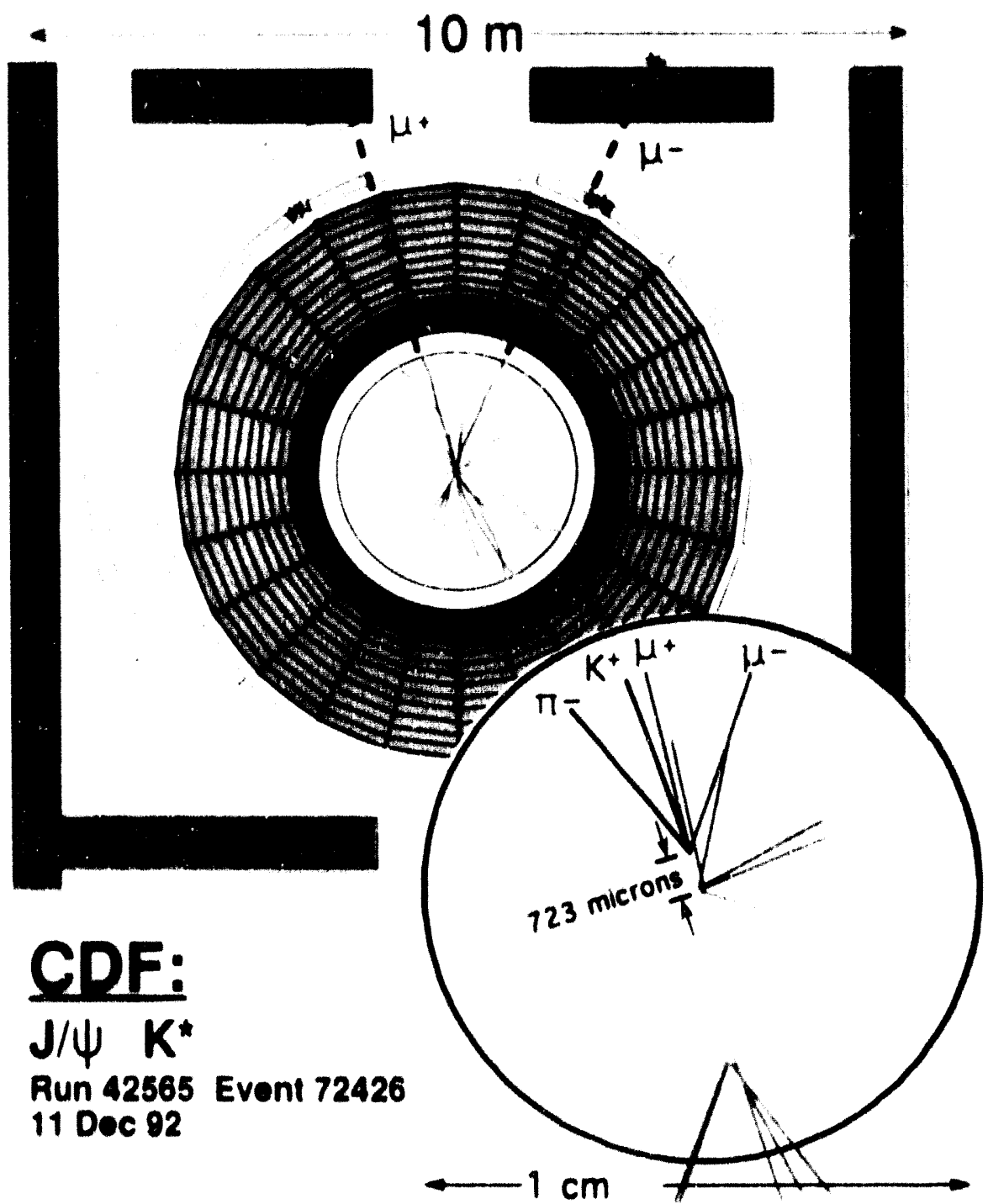


LBL has a major role in both of the two large detectors at the formilab Tevatron Collider, the Collider Detector Facility and the D-Zero. Both detectors are now laking data. The effectiveness of the Collider Detector Facility has been enormously enhanced by the Silicon Vertex Detector, for which LBL was the lead institution. This work involved close collaboration among the Physics Division, the Engineering Division, and industrial suppliers. The Silicon Vertex Detector has achieved approximately 10 micron resolution in the transverse plane. The result is that B physics has been opened up to an unprecedented and unexpected extent. The Silicon Vertex Detector may play a crucial role in the discovery of the I quark. LBL. groups are involved in analysis of $B$ decays, the $t$ quark search, and the measurement of the $W$ mass. Meanwhile, the D-Zero detector has started to produce physics remarkably quickly. LBL's contributions to the hardware, the VTX (Vertex Detector) and ECEM (End Cap Electromagnetic Modules) are functioning well. LBL groups are involved in analysis of $W$ and $Z$ events. The future of D-Zero lies with upgrades and LBL is heavily committed to the design and construction of a silicon vertex detector, building on the experience at LBL gained in the development of this type of detector. The SVXII chip is being developed jointly with Fermilab.

The SLAC Linear Collider has dramatically improved its performance. High luminosity running with good polarization is now regularly achieved. The LBL. Compton Polarimeter is a key element of this program. A stringent test of the Standard Model is being made by measuring the left-right asymmetry. Continued running with improved polarization will give a result that may rival those of CERN's Large Electron-Positron Colliding storage ring results for precision on the weak mixing angle.

The Astrophysics Program of the Physics Division has three components: cosmic microwave background measurements, a search for dark matter, and a search for distant supernovas. The cosmic microwave background program may be extended with new satellite-borne detectors. The dark matter search is embarked on a several-year program of experimentation. The distant supernova search has already discovered the most distant supernova ever observed. An expanded program should result in a few dozen distant supernovas, enough to determine the mass density of the universe.

\section{Theoretical Programs and Data Compilation}

The Laboratory has a strong theoretical particle physics group, whose work ranges from highly theoretical topics to others closely related to current experiments. A substantial effort is being devoted to theoretical studies in support of physics in the SSC energy range.

The Particle Data Group performs a service to the world's high-energy physics community through its compilations of particle properties. Its recent strengthening includes making databases more accessible through computer links.

\section{Detector Research and Development}

Advanced detector development is aimed at long-range research in detector problems relevant to proposed hadron colliders (see SSC below and Section 4). The program emphasizes the development of radiation-hardened devices, pixel devices, monolithic amplifier arrays, and data-acquisition electronics. 
A wedge-shaped silican microstrip) cletector for the D.Kers experiment is shown. fogether with its asoocialled reditout electronics. There are $B$ cusfom very large scale infegration IC, mourted on a low-mass kapton reddout circuit. which is in lurn mounted directly on the active silicon detector. Each 16 has $12 \mathrm{H}$ channels of electronics, for a total of 10.24 channets on this defector. Both the VLSI chip and the low-mass heplese circuit were developeed all $L B L$.
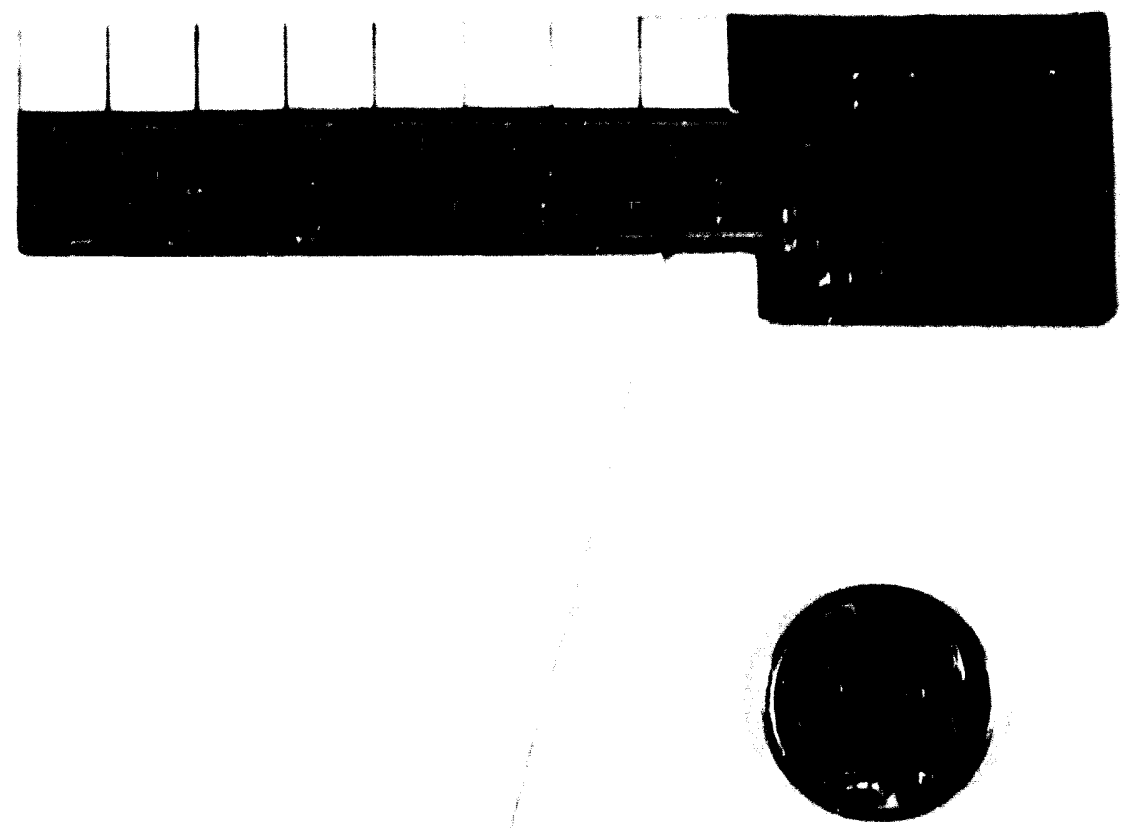

In addition, technology development efforts are directed toward ongoing detector construction and upgrade projects. For example, ultrahighresolution vertex detectors for MARK II at the SLC and D.Zero at the Tevatron I were fabricated to provide spatial resolution on the order of tens of micrometers to detect the decays of very-short-lived particles. Another example is the development of ultrahigh-resolution solid-state detectors to search for neutrinoless double beta decay to measure a finite neutrino mass.

\section{Accelerator Physics and Design}

Accelerator physics and engineering for the design and application of particle beams is an LBL core competency that has long played a leading role in research and development for high-energy physics facilities. In the recent past, for example, LBL experience with beam cooling resulted in successful systems at the Tevatron I. Most recently they have turned their attention to high-luminosity lepton colliders such as B-meson factories, and have been using their combination of analytical and practical expertise to solve a variety of problems, such as beam-beam instabilities and damping of higher-order modes.

A comprehensive, integrated approach to superconducting magnet $R \& D$ is an element of a competency that LBL applies to high-energy physics. The program emphasizes three major aspects of building higher-field and higherquality magnets. An ongoing effort to make wire for greater current density explores small filament diameters $(2-3 \mu \mathrm{m})$ and brittle superconductors such as niobium-tin. Improved cable designs are also a major focus of activity. These achievements come logether in the development of stronger and more efficient accelerator-type magnets, one of which set a field-strength record of slightly more than 10 tesla in 1992 testing. Other highlights include efforts to make better magnets and to find better ways of designing magnets. 


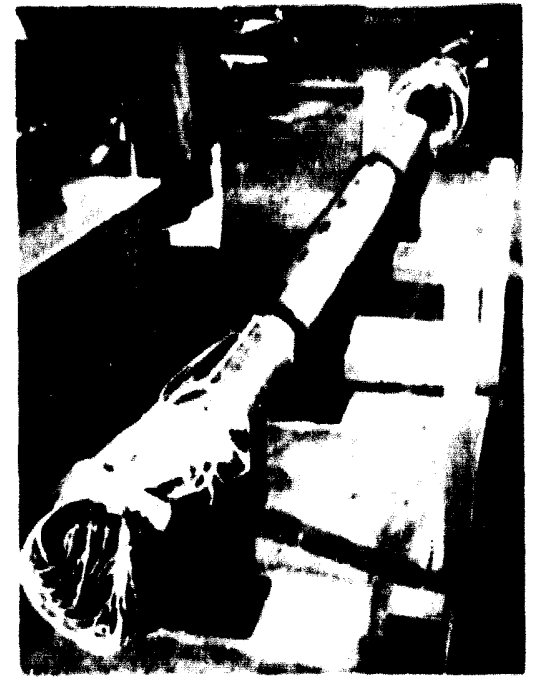

A prosetype SSC quadrupole magnet is inverted in its cryostat for testing. After a magnet has been fabricaled. it is operated in a cryostal all ever. increasing curremt until a "quench." l.e. a rapid herating and consequent loss of superronductivity. is detected. This intormation can be used to improve design and cabrication in the search tor grealeer reliability and predic ability.

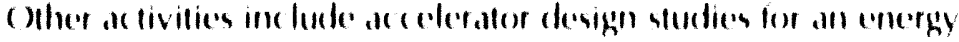

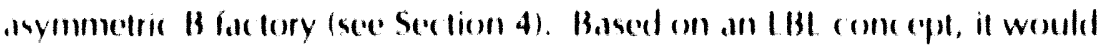
me ete collisions all different energies 10 produce B mesoms in a way that

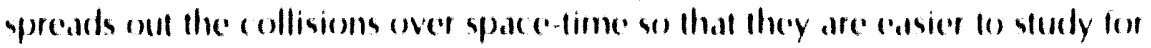
evidence of CP.violating dec ays.

In a futuristic program aimed at a next-generation high-energy electron collider, LBL scientists, collaboraling wilh LLNI and SLAC colleagues, are working on the Two-Beam Accelerator. This concept. pioneered at $|B|$. uses either a free-electron laser or a relativistic klystron to generalte extreme levels of microwave power, which are then applied to a high-gradient linac structure.

\section{Superconducting Super Collider}

LBL is conducting advanced detector development in detector problems directed specifically toward SSC physics. The program emphasizes the development of radiation-hardened devices, new pixel devices for twodimensional high-resolution detectors, and low-noise, high-speed monolithic amplifier arrays. Specific efforts are directed toward a silicon tracker, scintillation calorimetry, and front end electronics for the SSC. Cooperative efforts between LBL engineers and high-energy physicists are developing designs for improved data-acquisition electronics suibable for experiments at high-luminosity hadron colliders as a part of LBL's major Solenoidal Detecfor Collaboration initiative (see Section 4). The design, fabrication, and testing of SSC quadrupole magnets with a Nb-Ti superconductor continues at LBL.

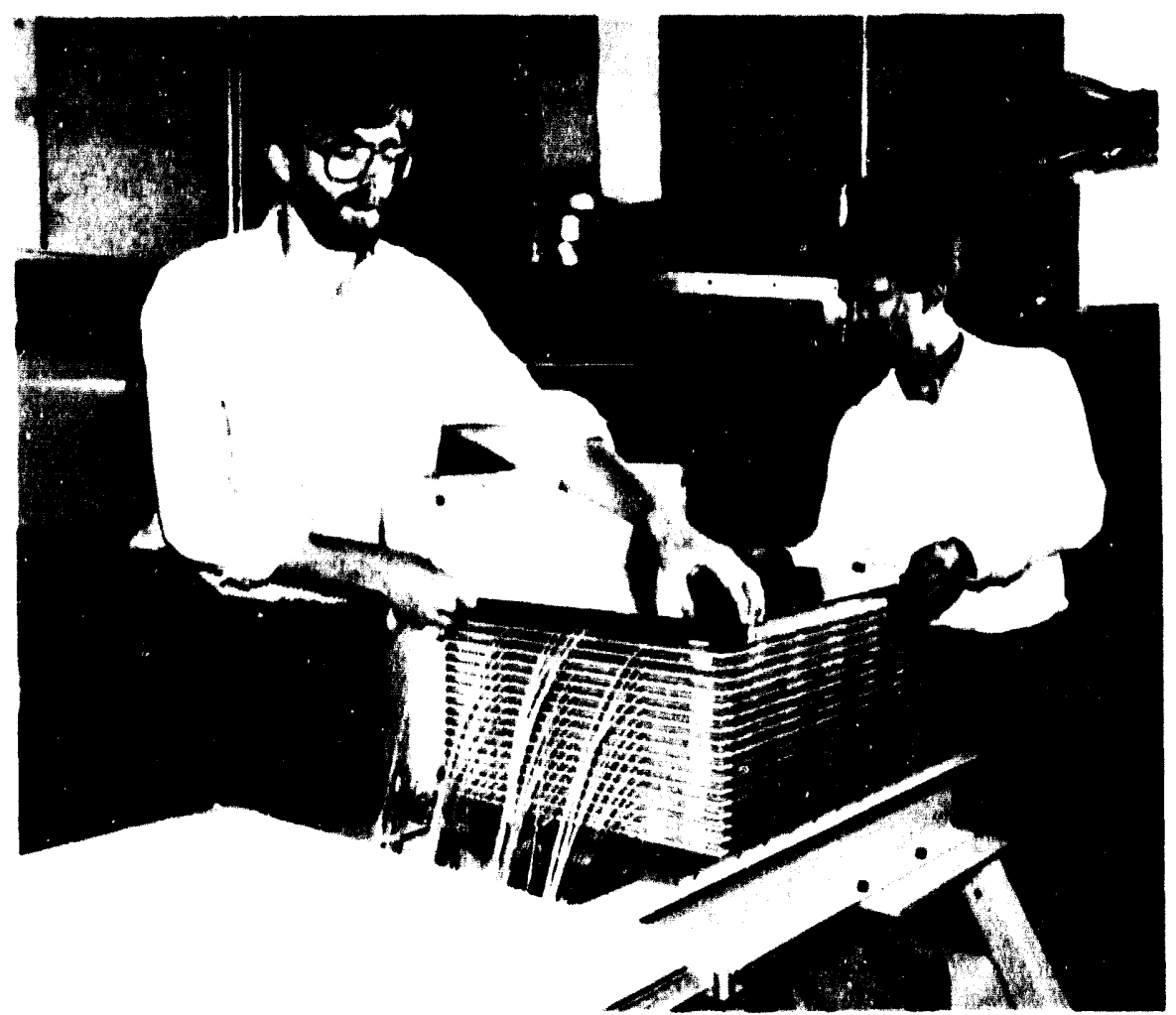
at LBL.
The prototype of the enctip electromagnetic calorimeter tor the SDC detector ot the SSC. Was assembled at L.BL. This prototype will be lested at CERN. The final SDC-SSC calorimeter is 10 be 54 meters in diameter and weigh 60 metric tons. 


\section{Office of Health and Environmental Research}

Life-sciences-related research activities include six research program areas: gene expression and genome mapping; structural biology; nuclear medicine and functional imaging; carcinogenesis, mulagenesis, and radiation biology: environmental and health-effects research; and measurement technology. These programs form a core of research conducted for DOE's national programs supported by OHER. Program expansions are anticipated in human genome research, structural biology, gene expression, growth regulation, molecular medicine, and environmental science.

\section{Gene Expression and Genome Mapping}

Important research growth areas for LBL are studies on human genome structure and regulation of gene expression. Research at the Human Cenome Center includes generation of physical and genetic maps at several levels of resolution $(20 \mathrm{~kb}$ to $100 \mathrm{~kb})$, identification and localization of all of the expressed genes on human chromosome 21 , and development of techniques and automation for efficient sequencing of human DNA.

As part ot the Human Genome Projert. LBL has concentrated on locating expressed genes on human chromosome 21. The schermaltic indicates that LBI. has more than doubled the number of genes located to specific regions of chromosome 21, as well as linking them to the yeast artificial chromosomes that form the backbone of the physical map of the

chromosome. This permits integraltorn of two kinds of maps: physical maps and expression gene) maps.

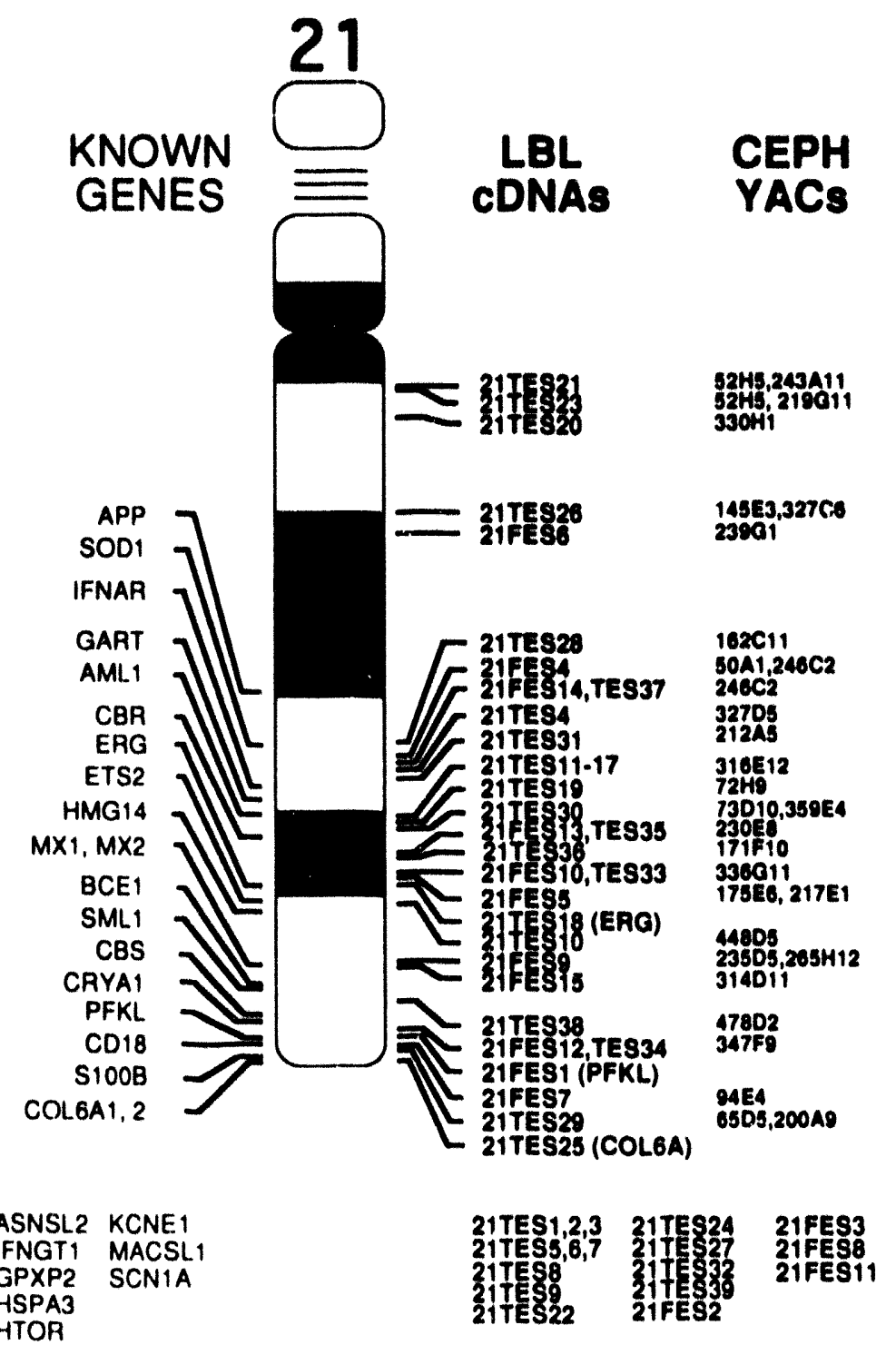


Analysis is under way of the biologically relevant signals culled from sequence information. Expressed genes and cDNAs are also being mapped throughout the Drosophila genome, in collaboration with UC Berkeley and Harvard University. Identification and mapping of groups of genes involved in the control of development and in the generation of personality traits are also part of the Center's program.

LBL's Life Sciences Division conducts several related research programs on gene expression within mammary-gland and blood-forming systems. The highly secretory mammary epithelial cells provide excellent models for gene expression and chemical- and radiation-induced carcinogenesis and are now also providing vehicles for production of genetically engineered foreign genes. LBL has identified hematopoietic research for expansion. Bloodforming cells are important targets of radiation-induced damage and are versatile models of stem-cell differentiation and regulation of gene expression.

Molecular cytogenetics describes a set of pioneering diagnostic methodologies for biomedical applications-many of which have already made a significant contribution to the megabase sequencing effort in the Human Genome Project. The research program centers on three core projects. In the first, refinements in hybridization technology are being made to increase the speed, sensitivity, and specificity of hybridization to allow rapid identification and mapping of genetic aberrations. Computer-assisted microscopy, the second emphasis, is being developed for rapid, high-resolution, multicolor imaging for the detection of rare genetic events. Probe development focuses on whole chromosomes, chromosome regions and specific repetitive DNA sequences. Probes will target specific regions of the human genome known to be involved in genetic disease. Fully integrated, the Center for Molecular Cytogenetics will develop improved methods of screening for prenatal and neonatal genetic abnormalities, cancer gene localization, and diagnosis and prognosis of solid tumors.

\section{Structural Biology}

One thrust of LBL's structural biology program is directed toward $x$-ray based research at the ALS, as described in Section 4. X-ray crystallography, electron crystallography, and NMR spectroscopy focus on protein and nucleic acid structures. Several studies are aimed at determining how proteins regulate the expression of genes by examining the structure of the protein-nucleic acid complexes. These latter studies will make use of advanced techniques made possible by the ALS such as x-ray diffraction.

Studies based on high-resolution electron crystallographic structure analysis and $x$-ray diffraction are also elucidating the structure of specific membrane proteins that are involved in transmembrane signaling. Using unique techniques for electron diffraction and imaging of crystalline sheets of membrane proteins, structural studies will be pursued on such membrane bound proteins as growth-factor receptors, chemotaxis receptors, and receptors for extracellular matrices. LBL's new intermediate-voltage electron microscope, which allows viewing of much thicker specimens than can be examined with conventional electron mi-roscopes, is enabling researchers to study complex structures, including that of the isolated mitotic spindle, which can be prepared as a frozen, hydrated specimen. Related work will exploit circular-dichroism microscopy, electron microscopy, and novel microscopic-imaging capabilities from the UV and SXR beams of the ALS. 
Hard x-ray radiation from a wiggler will make it possible to develop new technical capabilities in synchrotron-based protein crystallography that have never been attempted before. These include the use of specialized $x$ ray optical devices to provide multiplexing and time sharing capabilities for order-of-magnitude more efficient use of the available beams and $x$-ray microfocus technology to extend data collection capabilities to protein microcrystals as small as $20 \mu \mathrm{m}$ or less.

Current research is exploring new concepts in the realm of soft x-ray microscopy of biological specimens. The goal is to test ideas such as highresolution fluorescence microscopy (using lanthanide chelate labels) and the mapping of elemental distribution with in subcellular compartments, which could justify the construction of a scanning $x$-ray microscope. This microscope would use a specially designed undulator, optimized for light output in the so-called water window range of $x$-ray wavelengths.

LBL has also established a unique center of excellence, the Program for Biomolecular Design, that will bridge chemical, biological, and computer sciences by combining the expertise in LBL and the University of California, Berkeley. The Program will catalyze an understanding of biological systems with the ability to analyze and manipulate chemical structure. The aim is to redesign the natural biological molecules to create new classes of novel biomolecular structures with applications to major problems in the medical, biological and environmental sciences relevant to DOE missions.

\section{Nuclear Medicine and Functional Imaging}

Research in nuclear medicine will include studies involving molecular biology while continuing studies on improved radiopharmaceuticals and advanced instrumentation for applications to medical science. A systematic search for new, ultrafast heavy-atom scintillators will continue, as well as the development of solid-state photodetectors for multilayer high-resolution positron-emission tomography. A new multilayer, high-resolution tomograph design is planned for use in medical studies of the human brain and heart, as well as for studies in laboratory animals. This work includes studies of the causes of atherosclerosis and of the physiological basis of brain disorders, including Alzheimer's disease, using nuclear and organic chemistry, magnetic resonance and emission, tomographic imaging procedures, autoradiography, and advanced computer kinetic modeling. Highresolution in vivo microscopy of the human brain and in vivo carbohydrate metabolic studies are also being furthered through the development of advanced NMR instrumentation.

Methods for the production of radioisotopes and for the labeling of biochemical substrates to be used in noninvasive imaging have contributed to the effective use of these diagnostic-imaging tools. Newly developed radioisotope generators give greater flexibility to the application of shortlived, positron-emitting isotopes by using long-lived parent radioisotopes, absorbers, and elution techniques that favor the production of short-lived radionuclides.

A new Biomedical Isotope Facility that will provide isotopes for use by LBL scientists and other qualified users is under construction. The capability for using the short-lived light-element tracers from a minicyclotron that is the heart of the facility will be especially appropriate to a range of metabolic studies. The primary isotopes produced would be ${ }^{15} \mathrm{O},{ }^{11} \mathrm{C},{ }^{13} \mathrm{~N}$, and ${ }^{18} \mathrm{~F}$ for brain, heart, and cancer positron-emission tomography studies. The two 
A magnetic resonance image of a living human cortex from $L B L$ 's Center for Functional Imaging. Such images feature unprecedented resolution $(0.3 \times 0.3 \times 0.7 \mathrm{~mm})$ of $3-D$ in vivo structure. There are immediate clinical applications to epilepsy studies.

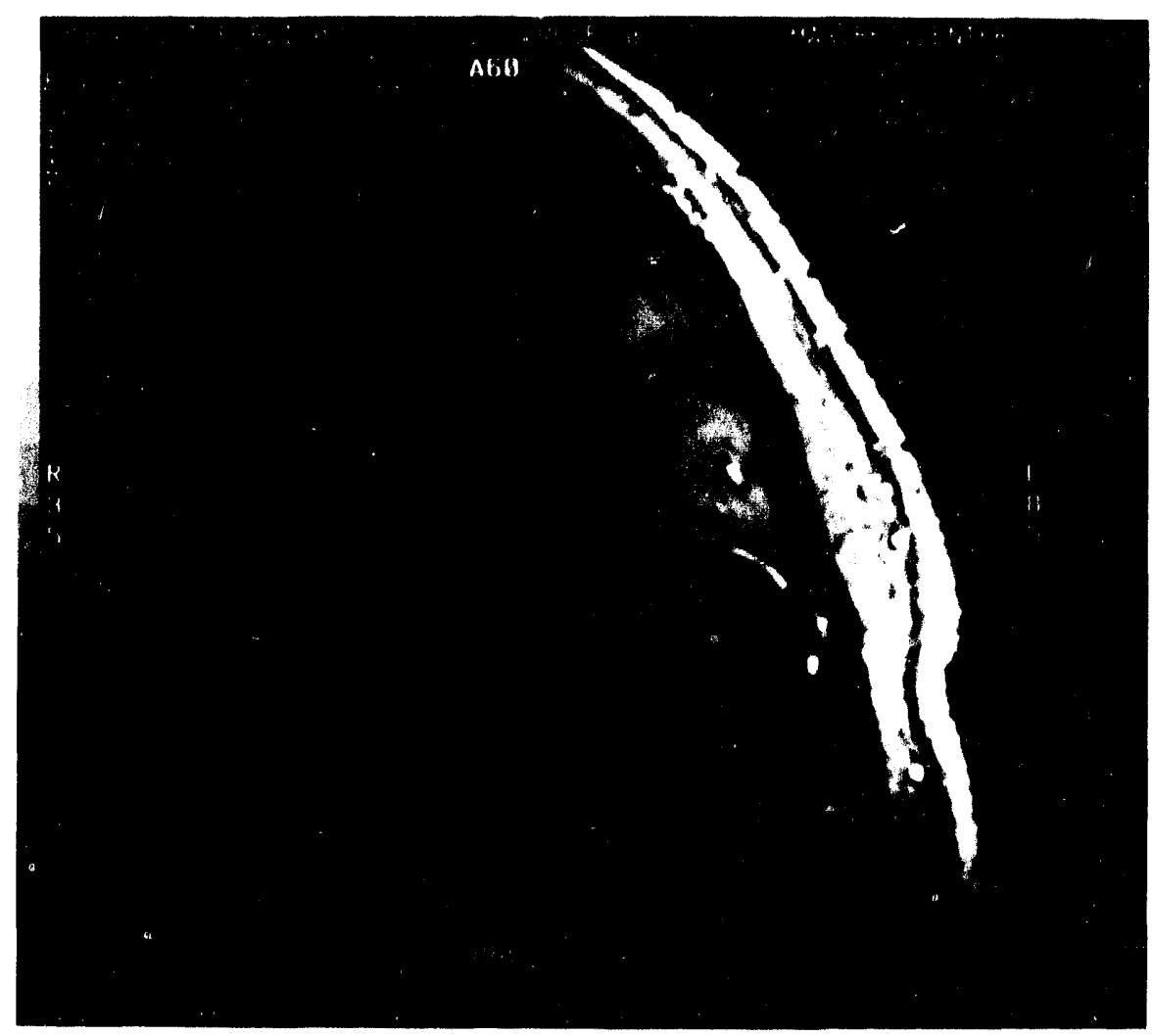

LBL positron tomographs, one with the highest spatial and temporal resolution in the world, will be utilized effectively with these short-lived positronemitting tracers.

In parallel with an emerging national trend in molecular nuclear medicine, studies in three new areas are in the works: (i) use of modern instrumentation and dosimetry, along with human genetic studies and transgenic animal models to pursue the relationship among variations in human low-density lipoprotein receptors, the genome, and the occurrence of atherosclerosis; (ii) use of advanced non-invasive methods of nuclear medicine and NMR to study the relationships among ion-channel protein aberrations, brain physiology associated with mental disorders, and the genome; (iii) use of ${ }^{13} \mathrm{C}-\mathrm{NMR}$ metabolic studies to evaluate the carbon cycle in plants relative to environmental changes in temperature, $\mathrm{CO}_{2}$, and nutrients.

\section{Carcinogenesis, Mutagenesis, and Radiation Biology}

LBL plans increased research emphasis on the role of oncogenes and tumor suppressor genes in cancer induction and embryogenesis, as well as on an integrated program in cancer initiation, promotion, and progression. Activities in the area of DNA damage and repair include cloning of human genes by complementation of repair defects in lower organisms, research on how structural constraints and organization of the cell's genome affect repair processes, studies of inducible responses to DNA damage, and cell culture studies of replication of damaged DNA templates. Studies using rodent and human epithelial and blood-forming cells in culture and in vivo are directed toward a better understanding of how differentiation and malignant transformation is controlled in their cells. Researchers are examining how the cells 

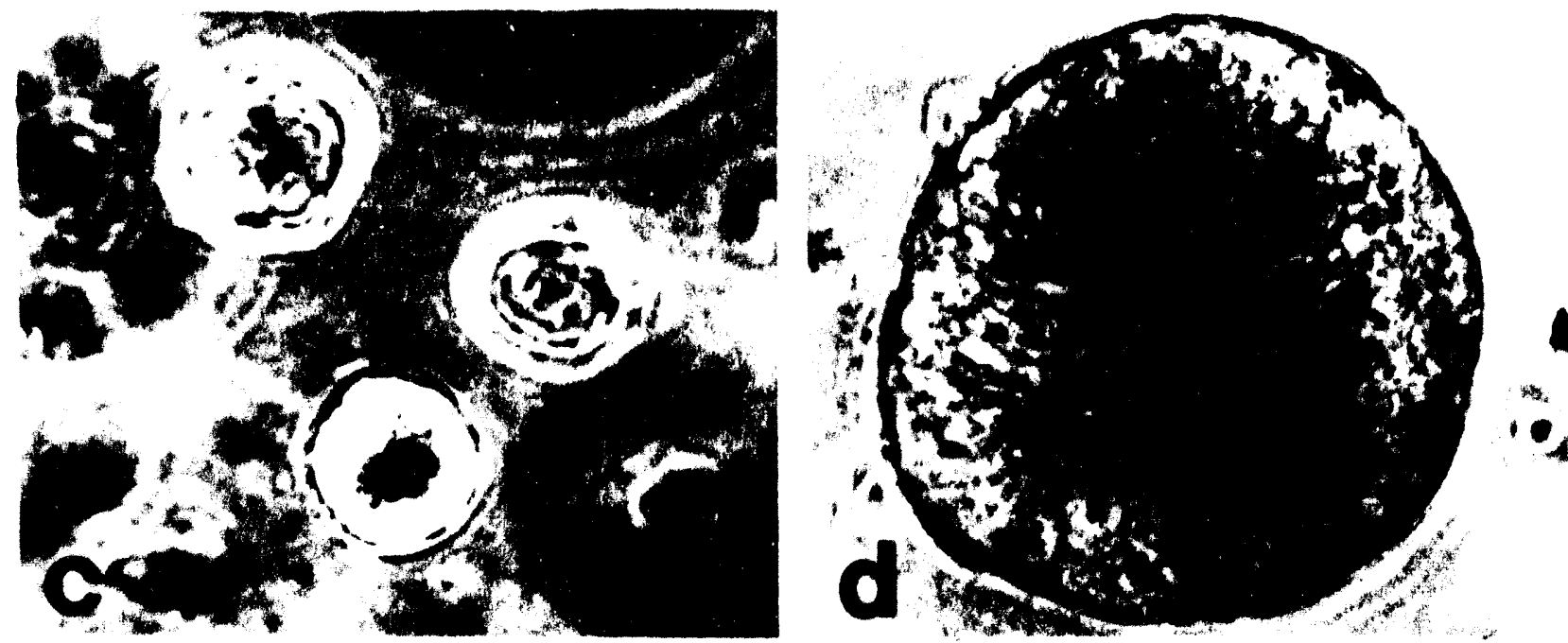

In collaboration with the University of Copenhagen, LBL scientists have developed a rapid culture assay to distinguish normal human epithelial breast cells from their malignant counterparts. This is achieved by using a basement membrane, a delicate noncellular matrix with which epithelial cells interact. The figures show the differential response of normal breast epithelial cells and carcinoma cells to basement membrane. The normal cell lines are shown to left, carcinoma cell lines are to right. communicate with their environment to regulate gene expression, growth, and differentiation. Using viral and cellular genes, they are studying critical features of oncogene and suppressor gene expression, including the influence of environmental variables, cellular growth factors, and cellular aging. Another area of research is directed at understanding how protooncogenes (which, through mutation, become oncogenes) and tumor suppressor genes (which become inactivated during carcinogenesis) function in normal, terminally differentiated, and neoplastic cells. Finally, researchers are intensively studying the process of hematopoiesis-the differentiation of blood cells from a multipotential stem cell-both because of its intrinsic significance and relationship to diseases of the blood and because it provides an excellent model for the processes that take place during embryogenesis.

LBL researchers are using electromagnetic and ionizing radiation ( $x$-rays and gamma rays) to evaluate the biological responses to these environmental hazards. Similar strategies are also being pursued with other carcinogens and mutagens (chemical oxidants) that are expected to generate information about the health consequences of environmental pollutants. Such work will enable continued progress in radiation medicine and the assessment of radiation and chemical hazards. The studies will attempt to separate the process of lesion formation in DNA from the processes of enzymatic repair. The influence of the extracellular environment, including hormones and the extracellular matrix, will be included in models of the relationship between exposure dose and biological effects. Another major effort entails resolving the differences in the responses of human and animal cells. Measuring the consequences of low-dose exposure will contribute to understanding the risks associated with accidental exposure to neutrons, space radiation, other occupational hazards, and exposure from medical diagnostic investigation.

\section{Environmental and Health-Effects Research}

Environmental research at LBL comprises multidisciplinary efforts on global, regional, and local environmental problems such as subsurface contamination, indoor air quality, and high-magnetic-field environments. 
Correlation analysis of indoor radon concentrations with soil radium and other physical parameters is being done at LBL. This will enable researchers to predict mean indoor radon concentrations for areas for which such data are not abailable and to identify regions of the U.S. with the highest indoor radon concentrations for remedial action. The map of Minnesota shows mean radon concentrations by county.
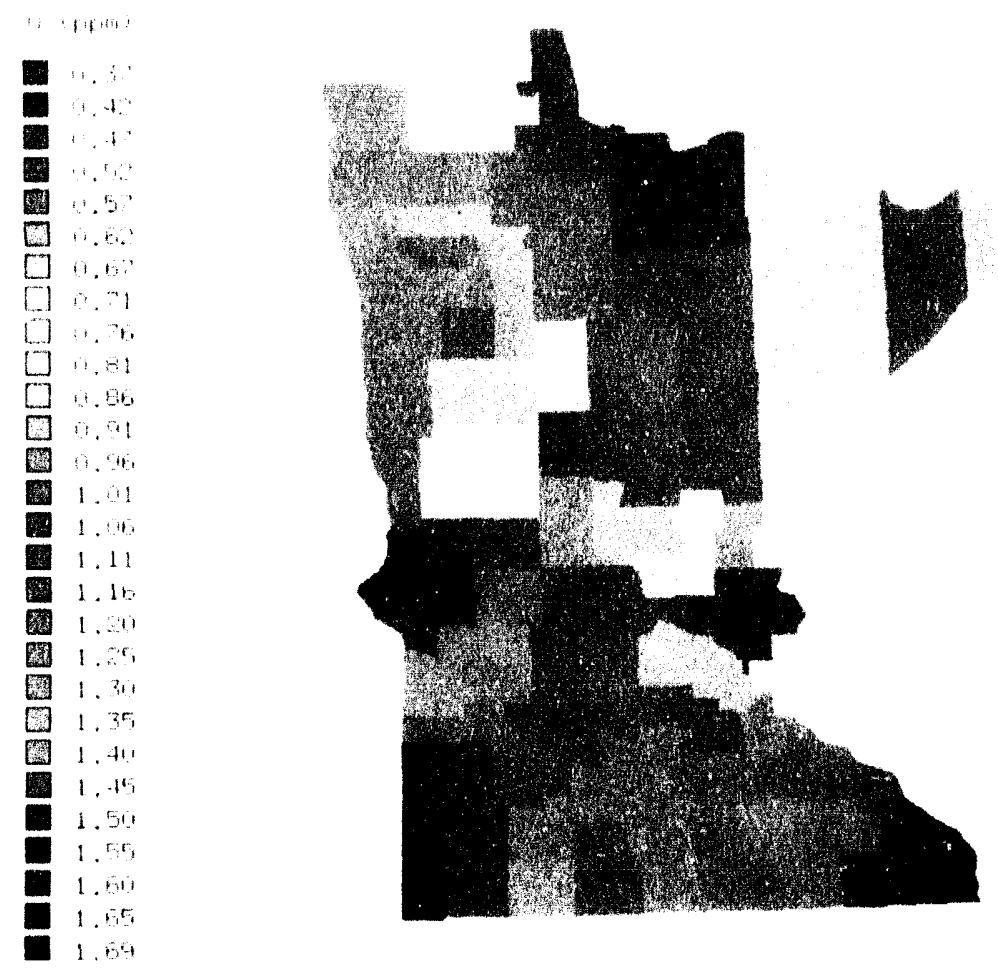

The Laboratory is advancing programs in support of DOE's research initiatives on the subsurface environment. The Laboratory's proposed program encompasses the biological and hydrogeochemical control and remediation of toxic waste. Specific projects include characterization of contaminants, subsurface heterogeneity, transport processes, and enhancement of restoration methods. Improved risk-estimation methods will enable the deployment of cost-effective remediation technologies.

LBL is developing an interdisciplinary program to investigate the processes that lead to changes in the physical and chemical characteristics of the atmosphere and other potential changes in the ecosystem. Initial research subjects include atmospheric processes that are involved in the generation of nucleating particles from artificial and natural sources; heterogeneous chemical processes and the role of particulates in the formation of clouds and the resulting chemical and physical changes in the atmosphere; and atmosphere-ecosystem interactions.

The $L B L$ program on radon migration includes unique experimental facilities for studying radon movement in soil and into buildings under controlled conditions, which will allow comparisons with detailed numerical simulation models. In addition, the radon research program examines the effects of other parameters, including other indoor air pollutants, building construction and operation, and climate conditions, on the resulting indoor radon concentrations and, ultimately, on the radiation doses to the building occupants from these indoor exposures.

\section{Measurement Technology}

Excellence in measurement technology is key to the success of OHER programs. Refinements in sensors and analytical techniques have been developed at LBL for a number of years. New detector configuration and 
the discovery of new scintillation will significantly benefit the positron emission tomography program of OHER. Research and development of the associated electronic signal-processing techniques complement this work. OHER support for $x$-ray detectors at the ALS and other synchrotron sources will utilize LBL expertise and provide significant advances in image resolution and chemical characterization of biological materials.

\section{Laboratory Information Management Systems}

LBL is developing data management tools for molecular biology laboratory information management system (LIMS) databases. These tools cover all aspects of developing LIMS databases, that is, database schema and integrity specification, data entry and manipulation, and browsing and querying LIMS databases. The goal of these tools is to ensure rapid development of flexible LIMS databases that can be easily modified and maintained, and to allow scientists to interact with the databases in terms of their own frame of reference, that is, LIMS-specific objects and protocols.

\section{Office of Fusion Energy}

Fusion energy research at LBL focuses on accelerator systems supporting the nation's inertial confinement and magnetic fusion energy programs. The LBL Heavy-Ion Fusion Accelerator Research (HIFAR) Group has focused its attention on exploring the physics and technology of induction acceleration as the means for producing high-current heavy-ion beams as a driver for inertial-confinement fusion systems. In comparison with other possible inertial-fusion drivers (e.g., lasers), beams of heavy ions offer important advantages for practical applications, including high efficiency from the "wall plug" to the beam, good potential for beam-to-pellet energy focusing and coupling, and high repetition rates. LBL's current efforts have resulted

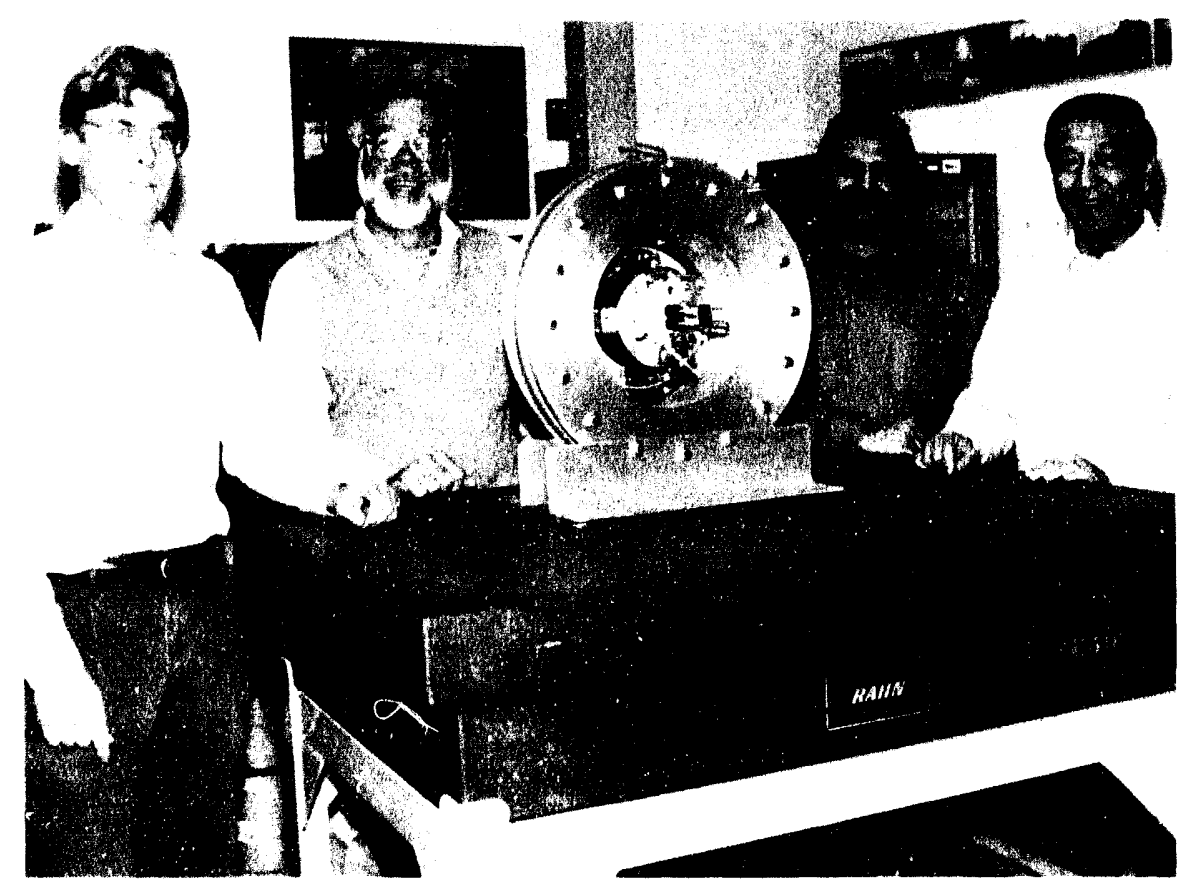

A negative ion source designed and built at $L B L$ has been sent to the SSC Laboratory to test the radio frequency quadrupole of the injector, and will be evaluated for possible use as the main ion source for the SSC itself. 
in successful completion of the multiple-beam experiment to examine the initial accelerator components for space-charge-dominated beams undergoing current amplification. Current efforts are directed toward design and development of the next set of studies, the Induction Linac Systems Experiments (ILSE), as described in Section 4. The ILSE accelerator would constitute a significant step toward realizing induction accelerator technology that could be used to produce intense sources of spallation neutrons. An induction accelerator that uses $5-\mathrm{MW}$ beams of protons at $1 \mathrm{GeV}$ to generate spallation neutrons in 100-ns bursts at a rep-rate of $10 \mathrm{~Hz}$ would be quite similar but one-tenth the size and cost of that needed for an inertial fusion driver. At higher average power levels (i.e., rep-rate) induction accelerators are candidates for accelerator-based pulsed reactors for waste transmutation (ATW), tritium production (ATP), and for a Fusion Material Test Facility.

$\mathrm{LBL}$ contributes to the magnetic-confinement fusion program through the development of neutral beams for heating, refueling, and confining reactor plasmas. LBL's work on positive-ion-based neutral beams has been coupled with the research efforts at the Tokamak Fusion Test Reactor at Princeton and the DIII-D at General Atomics in La Jolla. Studies for an Engineering Test Reactor, specifically the International Thermonuclear Experimental Reactor (ITER), have identified neutral beams as a candidate for driving current in the steady state. The mission of ITER, and the need for neutral beams, will be re-examined during the initial part of the next phase of the project, the Engineering Design Activity. As a result, research and development of high-energy ( $\geq 1 \mathrm{MeV}$ ) beams for heating and current drive in ITER may increase. Another possible future application of high energy neutral beams would be as an update of the neutral beam system of the proposed Tokamak Physics Experiment (TPX). These systems will be based on the production and acceleration of negative deuterium ions.

An additional contribution to the magnetic fusion program in the Materials Sciences Division is research on alloys and weldments for lowtemperature superconducting magnets for magnetic-confinement fusion systems. The alloys must withstand extremely high magnetic fields at the cryogenic temperatures needed for superconductivity.

\section{University and Science Education Programs}

In support of DOE's role in energy-related science education and technology transfer, LBL conducts training and technology-transfer activities with many organizations and institutions as part of the Laboratory's mission to educate and train scientists and engineers and to transfer knowledge and technological innovations to industry. These programs, currently undergoing significant expansion in response to strategic national goals for investment in human resources and long-term economic development, include collaborative technology research projects with industry, an industrylaboratory-personnel exchange program, and science education activities (see Section 7). 


\section{ENERGY EFFICIENCY AND RENEWABLE ENERGY}

The LBL program in Energy Efficiency and Renewable Energy comprises a broad set of related activities that provide research support and technology development in support of national goals to reduce energy demand and cost to consumers, balance environmental concerns with economic development, and enhance energy security. LBL's programs are principally in electrical energy storage and distribution, buildings, industry, transportation, utility systems, and geothermal systems.

Energy Efficiency and Renewable Energy Funding Summary

(Fiscal Year Operating and Capital Budget Authority, \$M)

\begin{tabular}{lrrrr}
\hline Major Program (BR Code) & 1992 & 1993 & 1994 & 1995 \\
\hline Buildings Sector (EC) & 9.3 & 10.7 & 15.2 & 15.9 \\
Electric Energy (AK) & 0.2 & 0.2 & 0.2 & 0.2 \\
Geothermal (AM) & 1.5 & 0.9 & 0.9 & 1.0 \\
Industrial Sector (ED) & 1.4 & 1.2 & 1.2 & 1.3 \\
State \& Local Assistance (EF) & 0.0 & 0.1 & 0.2 & 0.2 \\
Transportation Sector (EE) & 3.0 & 4.0 & 4.0 & 4.2 \\
Utility Sector (EK) & 0.8 & 1.0 & 1.1 & 1.1 \\
Total & 16.2 & 18.1 & 22.8 & 23.8 \\
Percent of LBL Total & 5.6 & 6.9 & 7.4 & 7.5 \\
\hline
\end{tabular}

\section{Utility Technologies}

LBL's programs provide assistance to utility planning and renewable energy supply, and research that will yield long-term options for utilities. An expanding effort in Integrated Resource Planning is directed toward providing information and analytic methods for incorporating conservation and renewable options in utility planning on an equivalent basis to conventional supply options. This effort is carried out in close collaboration with the National Association of Regulated Utility Commissioners.

The work on electrochemical energy storage, described below under Transportation Technologies, will provide stationary energy storage options for load-leveling applications. In another effort, work is ongoing to understand the effects of electromagnetic fields on biological systerns.

A multidisciplinary program addresses the characterization and development of geothermal energy resources. The current program consists of field, laboratory, and theoretical studies covering four principal technical areas: delineation and evaluation of geothermal systems, definition of reservoir processes, modeling of reservoir dynamics and exploitation effects, and optimization of field management practices. 
An LBL experimenter uses a polarization nepholometer to investigate the properties of aerogel, an inert super-insulating material. Aerogels have four to five times improvement of insulating value over polyurethane foam. They are relatively transparent, so are good possibilities for improved windows.

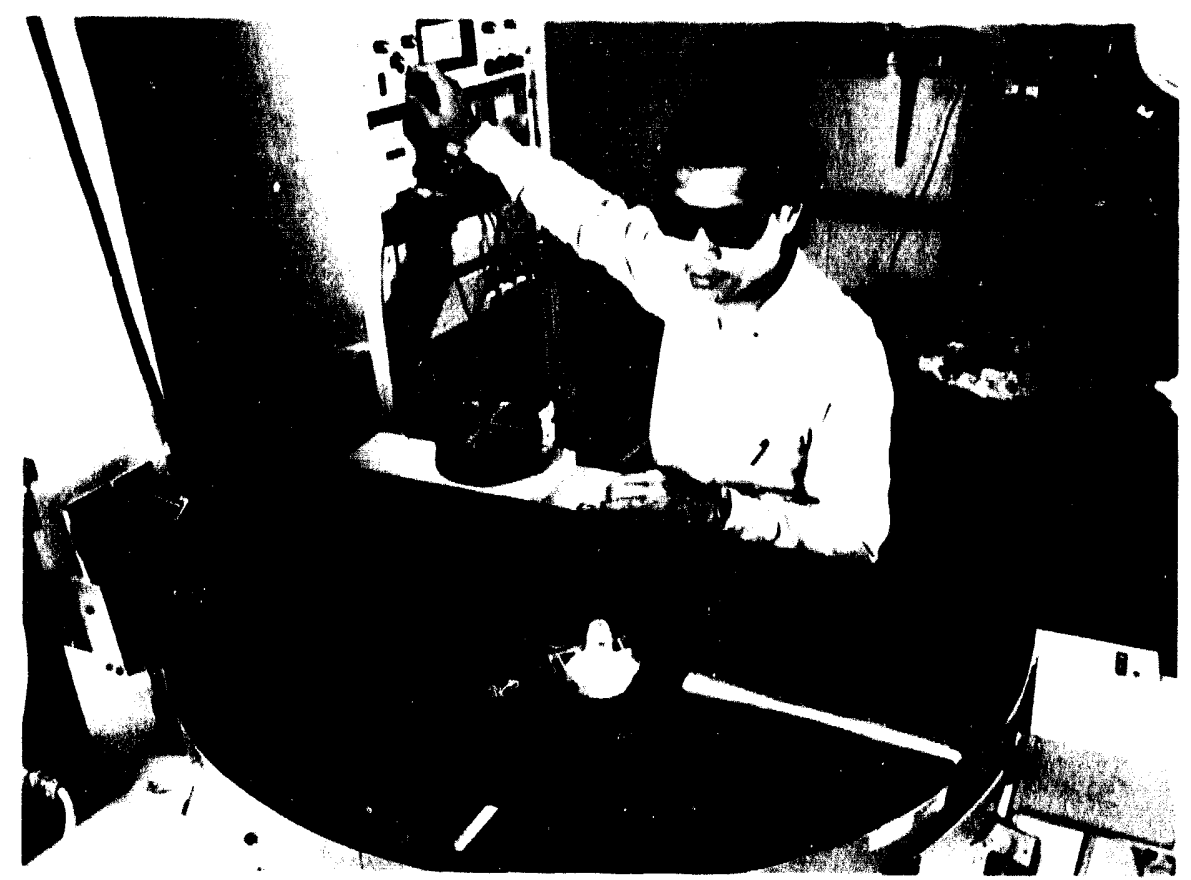

Reservoir technology work will lead to more accurate predictions of the responses of a geothermal reservoir to exploitation for optimum management through carefully designed fluid-production and injection operations. Joint field projects with U.S. geothermal developers and utilities continue to be highly productive, as do collaborations with organizations in Mexico, Iceland, and Italy.

Currently, LBL is investigating The Geysers geothermal field-the largest in the world-as well as several other systems in California and Nevada as part of a DOE-industry multi-institutional collaboration.

Magnetic-field interactions are being evaluated in experimental-animal systems and in tissue and cellular systems potentially sensitive to this nonionizing radiation. This program will develop theoretical models of magnetic-field interactions with biological systems and provide essential data for assessing the potential effects of magnetic fields.

\section{Industry Technologies}

This effort focuses on advanced industrial concepts, including energyefficient chemical separations such as distillation, the opacification of aerogels for high-performance insulation in non-view applications (e.g., refrigeration), and premixed lean-engine combustion, which holds the promise of reduced emissions, improved engine efficiency, and extended fuel tolerance. Work is underway in two projects on catalysts for industry: theory-assisted design of metal and zeolite catalysts; and chemistry, immunology, and modeling as tools for the rational design of stable, active enzymes.

\section{Transportation Technologies}

LBL manages the Exploratory Technology Research Program, which is the electrochemical research arm of DOE's Office of Propulsion Systems. The primary goal of this program is to advance the development of highperformance rechargeable batteries and fuel cells for use in electric vehicles. The battery performance goals for this program have been established by the 
Flexible gas filled panels, developed at $L B L$, can be used for stud wall insulation in manufactured or conventional housing applications, as well as automobile insulation in body design. Several licensing agreements are under discussion at this time with a variety of industrial partners. Popular Science magazine awarded the gas-filled insulation one of its grand winners for best new building technology.

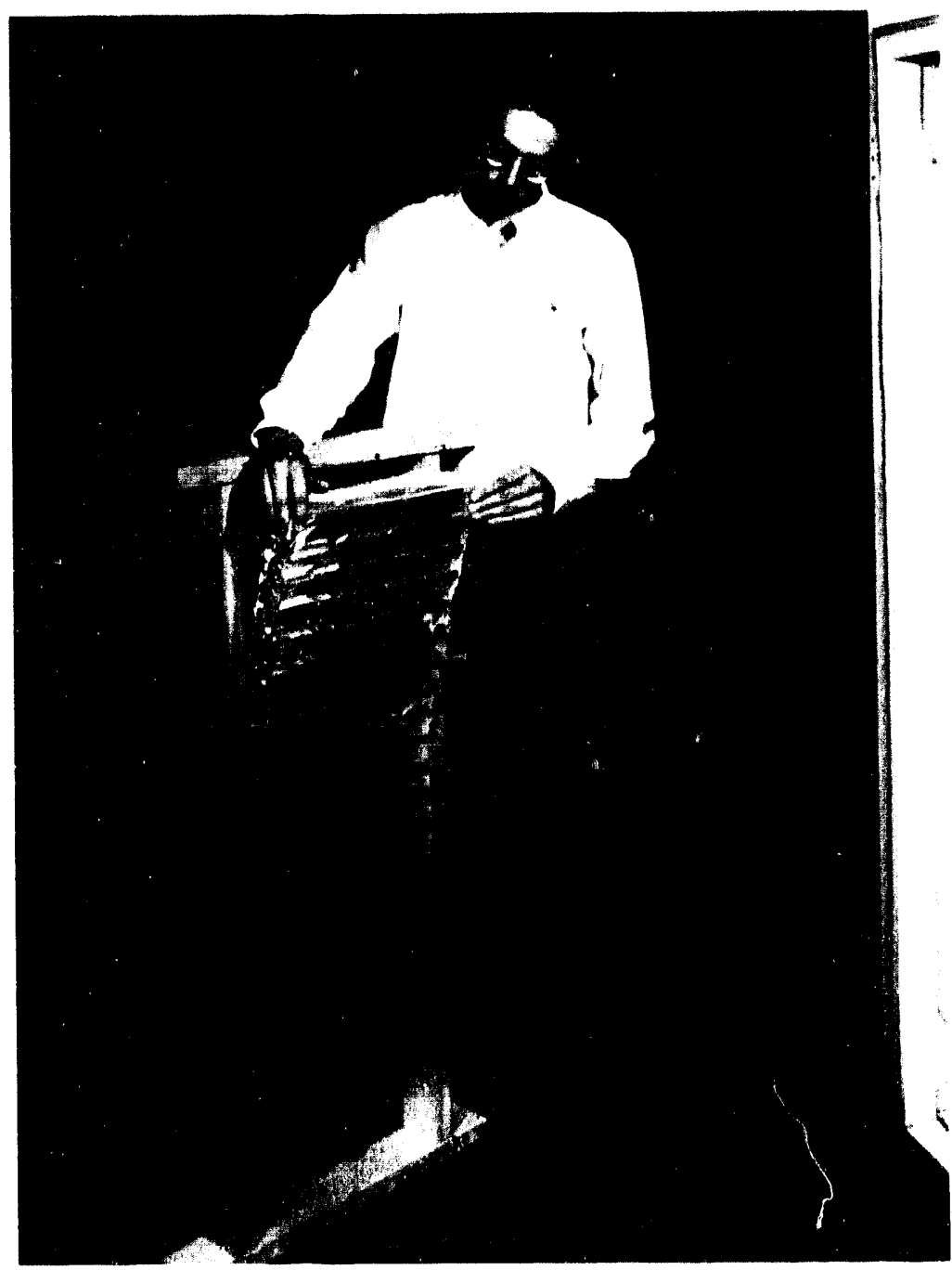

U.S. Advanced Battery Consortium. LBL research areas include exploratory R\&D on new electrochemical systems, supporting research for advanced rechargeable batteries currently under development, materials science research for improved battery components, fundamental electrochemical research to improve electrochemical energy conversion efficiency, and the characterization of advanced electrodes and electrolytes for use in fuel cells.

Based on its work for Building Technologies, LBL is developing switchable glazings for automobiles and other vehicles. Optical control to reduce solar heat gain will permit down-sized air conditioners and thus reduced use of CFC refrigerants as well as increased mileage.

\section{Building Technologies}

LBL will continue activities related to residential and commercial buildings in a program of laboratory and field research, modeling, and data analysis. This work is a coordinated systems approach to designing building components and whole buildings with improved energy efficiency. Modeling and field measurements verify results on economic costs and benefits of conserving energy efficiently. Important aspects of the work include measurements of indoor air quality and possible health effects of proposed efficiency measures. The initiative on Advanced Energy Design and Operation Technologies (see Section 4) is an extension of these efforts.

The Laboratory has a lead role in applied research in four areas related to energy efficiency in buildings: windows and daylighting, artificial lighting, computer modeling of building energy use, and infiltration/ventilation and indoor air quality. The general objective of these programs is to develop advanced energy efficient technolo. gies and to develop computational tools and experimental facilities. This will allow evaluation of technologies showing the greatest promise for significant energy savings in buildings while maintaining levels of illumination and air quality adequate for human comfort and health. Specific projects focus on energy-savings opportunities in fluorescent lamps, advanced windows, novel building insulation, analysis of federally assisted housing, residential and commercial building analysis and performance studies, analysis of appliance energy efficiency, and site-planning studies to minimize summer heat-island effects. These projects are undertaken in close collaboration with the building industry and utilities.

Both domestic and international studies of economic impacts of alternative energy policies are expected to grow. The purpose of surveying the conservation policies of other developed countries is to enable the U.S. to compare progress in this area and perhaps adopt effective efficiency measures. 


\section{OFFICE OF FOSSIL ENERGY}

LBL conducts research directed toward making coal more usable, including studies on conversion to gaseous and liquid fuels and reduction of emissions. One current effort focuses on the low-temperature catalytic gasification of graphite and other forms of carbon. A flue-gas chemistry project is directed toward methods of simultaneous removal of $\mathrm{SO}_{2}$ and $\mathrm{NO}_{\mathbf{x}}$, and other new processes are being developed to remove $\mathrm{H}_{2} \mathrm{~S}$ from gas streams, such as those produced during coal gasification. Another project is studying the erosion and corrosion of materials used in systems developed for coal conversion and use.

A study is under way to develop methods for characterizing heterogeneous and fractured reservoirs through a combination of geological, geophysical, and hydraulic studies. Heuristic methods are used to determine the systematic patterns that result from the geologic processes. Improved methods are being developed for imaging these features from boreholes. Both of these types of information are used to obtain a model of reservoir performance. This new approach results in a model that is a co-interpretation of all reservoir data and consequently should provide a much-improved ability to manage reservoir production.

Fossil Energy Funding Summary

$L B L$ researchers have developed a highly efficient technology for scrubbing flue gas that, in benchscale results, can remove better than $95 \%$ of sulfur dioxide and nitrogen oxides from coal furnace exhaust. Nicknamed PhoSNOX, the method uses yellow phosphorus, a novel scrubbing agent, with wet limestone to initiate a series of reactions that result in nearly pollutant-free emissions. The method is highly attractive and cost-effective.

Wot PhoSNOX Process

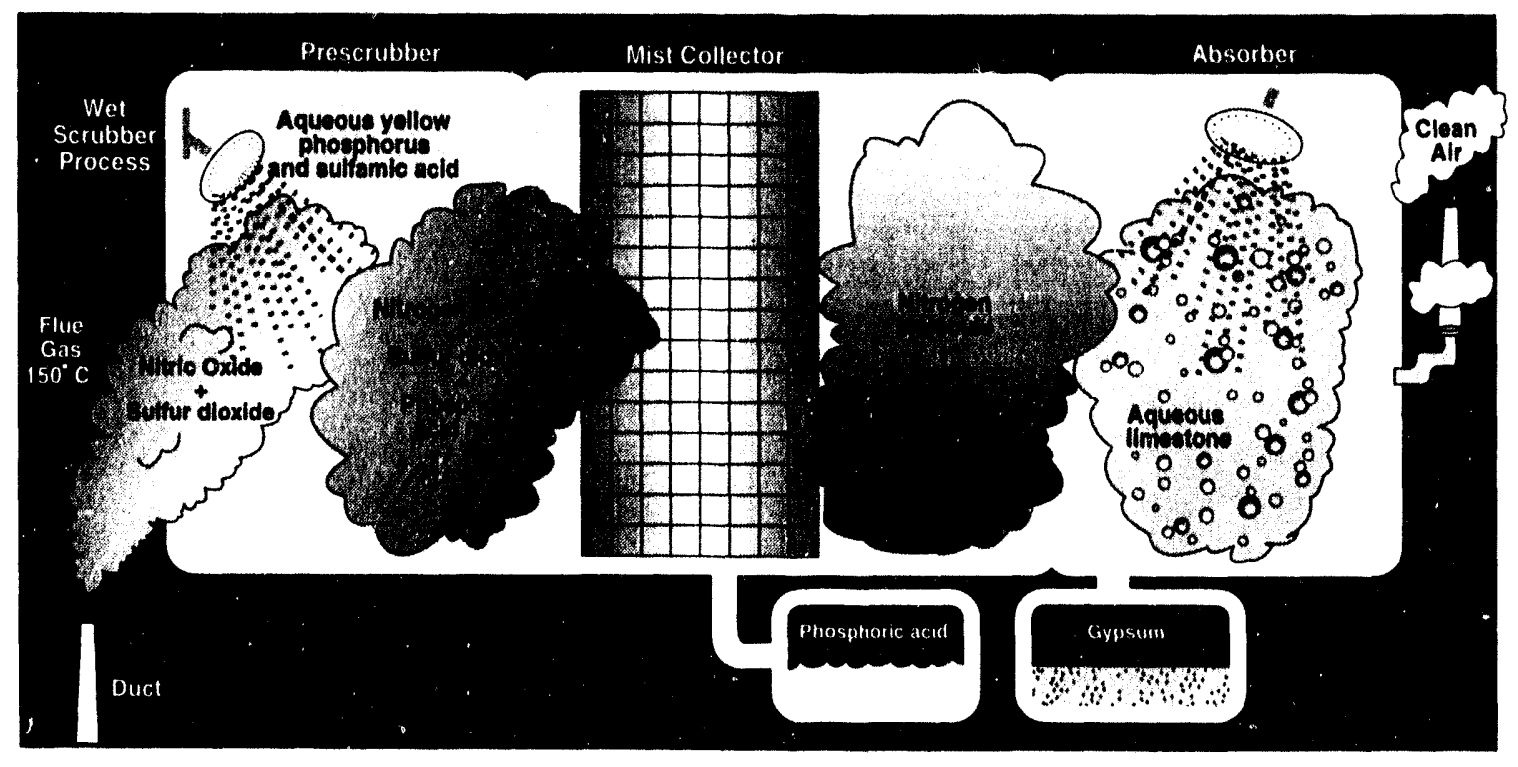




\section{ENVIRONMENTAL RESTORATION AND WASTE MANAGEMENT}

The Laboratory is implementing site projects for restoration and waste management consistent with DOE's National Environmental Restoration and Waste Management Program. As described in greater detail in Section 6 , the existing and budgeted site projects address specific conditions at the Laboratory, including facilities and operating programs for corrective actions, environmental restoration, and waste management.

Funding dropped from FY 1992 to FY 1993 because LBL's Corrective Action Plan is no longer funded through ERWM. The increase in FY 1994 is due to the construction start of the Hazardous Waste Handling Facility.

Environmental Restoration and Waste Management Funding Summary (Fiscal Year Budget Authority, SM)

\begin{tabular}{lrrrr}
\hline Major Program (BR Code) & 1992 & 1993 & 1994 & 1995 \\
\hline Environmental Management (EX, EW) & 14.7 & 10.4 & 20.7 & 18.8 \\
Percent of LBL. Total & 5.1 & 4.0 & 6.7 & 5.9 \\
\hline
\end{tabular}

"Includes costs with reference to Section 6.

\section{ENVIRONMENT, SAFETY, AND HEALTH}

LBL is continuing its strong programs in analytical methods development and statistical studies of environmental and epidemiological factors supported by the Office of Epidemiology and Health Surveillance. The Population at Risk to Environmental Pollution project focuses on the collection, analysis, and interpretation of data pertaining to relationships between human health and environmental pollution. Computational techniques are developed for the analysis of ecologic data, especially small-area geographic data, to investigate alleged departures from expected disease rates, to generate etiologic hypotheses, and to plan clinical trials or cohort studies. The role of the Comprehensive Epidemiologic Data Resource project is to provide exposure and health data on DOE workers to research epidemiologists both within and outside DOE.

Environment, Safety and Health Funding Summary

(Fiscal Year Budget Authority, \$M)

\begin{tabular}{lcccc}
\hline Major Program (BR Code) & 1992 & 1993 & 1994 & 1995 \\
\hline Environmental Health \& Safety (HA) & 1.8 & 2.6 & 2.6 & 2.7 \\
Percent of LBL Funding & 0.6 & 0.8 & 0.8 & 0.9 \\
\hline
\end{tabular}




\section{OTHER DOE PROGRAMS}

LBL maintains active research programs in Civilian Radioactive Waste Management, Policy Planning and Analysis, In-House Energy Management, and in supporting other DOE contractors, such as the Superconducting Super Collider Laboratory.

Other DOE Programs Funding Summary

(Fiscal Year Budget Authority, \$M)

\begin{tabular}{lrrrr}
\hline Major Program (BR Code) & 1992 & 1993 & 1994 & 1995 \\
\hline $\begin{array}{lrrr}\text { Civilian Radioactive Waste } \\
\quad \text { Management (DB) }\end{array}$ & 3.5 & 3.3 & 3.4 & 3.6 \\
$\begin{array}{l}\text { Policy, Planning, \& Analysis (PE) } \\
\text { Administration and Human }\end{array}$ & 1.6 & 1.5 & 2.0 & 2.1 \\
$\quad$ Resource Management (WB) & 5.3 & 1.1 & 2.3 & 2.3 \\
Other DOE Contractors (WFO) & 16.8 & 14.2 & 17.6 & 15.5 \\
Total & 27.2 & 20.1 & 25.3 & 23.5 \\
Percent of LBL Total & 9.4 & 7.7 & 8.2 & 7.4 \\
\hline
\end{tabular}

\section{Civilian Radioactive Waste Management}

LBL continues a strong multidisciplinary program of interrelated geoscience and geological engineering research important to the safe long-term underground storage of high-level nuclear wastes, e.g., characterization of deep geologic formations, determination of the physical and chemical processes occurring in the repository rocks, analysis of hydrologic and chemical transport mechanisms, and development of predictive techniques for repository performance. Coupled with ongoing basic research, $L B L$ is contributing to technology and applied development research at DOE's Yucca Mountain Project as well as to international projects in cooperation with Sweden, Switzerland, Canada, and Japan.

Experimental work involves testing rock samples to determine fundamental chemical, mechanical, and hydrologic parameters under a suite of anticipated repository conditions. Complementary research is conducted on the solubilities of actinides and the characteristics and processes that control radionuclide transport in host rocks. Related efforts involve understanding of processes and the development of methods for predicting the response of geologic systems to repository development and the performance of geologic environments for various repository containment designs. These expanding research activities draw upon LBL's expertise in chemistry, earth science, computing, and numerous engineering fields.

\section{Policy, Planning, and Analysis}

LBL undertakes analysis activities in support of policy issues of concern to DOE. Recent efforts include analysis of "feebates" (an imposition of fees and concomitant offering of tax incentives to encourage energy efficient technologies) as a policy approach to increase auto fuel economy, combus- 
tion pollution exposure that takes place indoors, the development of data and models for developing projections of energy demand under a variety of policy cases, and assessment of a variety of policies as an input to DOE.'s assessment of U.S. energy strategies. An ongoing task related to important national energy issues is to assess the performance of utility demand-side management programs, to gather better cost data for energy efficiency measures, and to incorporate this information into forecasts of residential and commercial energy demand.

In support of DOE's interest in developing a comprehensive understanding of factors that influence the release of "greenhouse" gases, LBL is undertaking a series of studies on global energy demand and related issues. Included are (1) an extensive analysis of China's energy system and an exchange and collaboration program with the Energy Research Institute of the People's Republic of China; (2) an analysis of electricity and overall energy demand growth patterns in the major developing countries; (3) energy demand analysis of Poland; (4) development of technology database for the Intergovernmental Panel on Climate Change; (5) and a review of current knowledge about selected environmental parameters in developing countries. An initial effort to combine this knowledge into a set of global scenarios is under way.

\section{Administration and Human Resource Management}

LBL's In-House Energy Management program pursues opportunities to significantly reduce energy costs at LBL. It is estimated that the program will result in over $\$ 2$ million in annual savings. The program involves surveys and studies of existing conditions, retrofit projects, new construction, and utility management and related operational programs. Recent major projects have improved accelerator efficiency, lighting, and utility systems.

\section{Other DOE Contractors}

LBL conducts research and development programs in support of the SSC Laboratory, including magnet systems technology development and research on detectors (see Superconducting Super Collider above). The magnet systems work, now focusing on dipole magnets, is supported through the Universities Research Association, the SSC Laboratory contractor.

LBL contributes to the research programs at other DOE national laboratories and facilities through such activities as management and design for the STAR project at Brookhaven National Laboratory (see Relativistic HeavyIon Physics above), laser-material interactions for Lawrence Livermore National Laboratory, development of specialized object scanning CCD cameras, the investigation of advanced windows and energy-conservation strategies for the Bonneville Power Administration, and environmental research and technology developrnent at DOE sites.

\section{WORK FOR OTHERS}

LBL WFO complements DOE research programs and provides unique research resources to other agencies and organizations. Support from some federal agencies is being complemented by growing collaborative research partnerships with the private sector in support of national terhnology transfer goals, discussed in more detail in Section 7. 
Work for Others Funding Summary

(Fiscal Year Budget Authority, \$M)

\begin{tabular}{lrrrr}
\hline Agency & 1992 & 1993 & 1994 & 1995 \\
\hline National Institutes of Health & 14.3 & 15.9 & 16.9 & 17.8 \\
Department of Defense & 12.8 & 12.2 & 30.0 & 20.0 \\
National Aeronautics \& Space Adm. & 3.2 & 3.2 & 2.7 & 2.6 \\
Environmental Protection Agency & 2.0 & 1.3 & 1.6 & 1.8 \\
Department of the Interior & 0.7 & 0.6 & 0.7 & 0.7 \\
Other Federal Agencies & 1.3 & 0.6 & 0.8 & 0.8 \\
State and Private & 12.0 & 17.4 & 15.8 & 15.6 \\
Total & 46.3 & 51.2 & 68.5 & 59.3 \\
Percent of LBL Total & 16.0 & 19.6 & 22.2 & 18.8 \\
\hline
\end{tabular}

A gene that predisposes individuals to atherosclerosis, the leading cause of heart disease in this country, has been discovered by scientists at $L B L$ and the Children's Hospital Oakland Research Institute. Designated ATH-S, for atherosclerosis susceptibility, the gene was located on chromosome 19.

\section{CHROMOSOME 19}

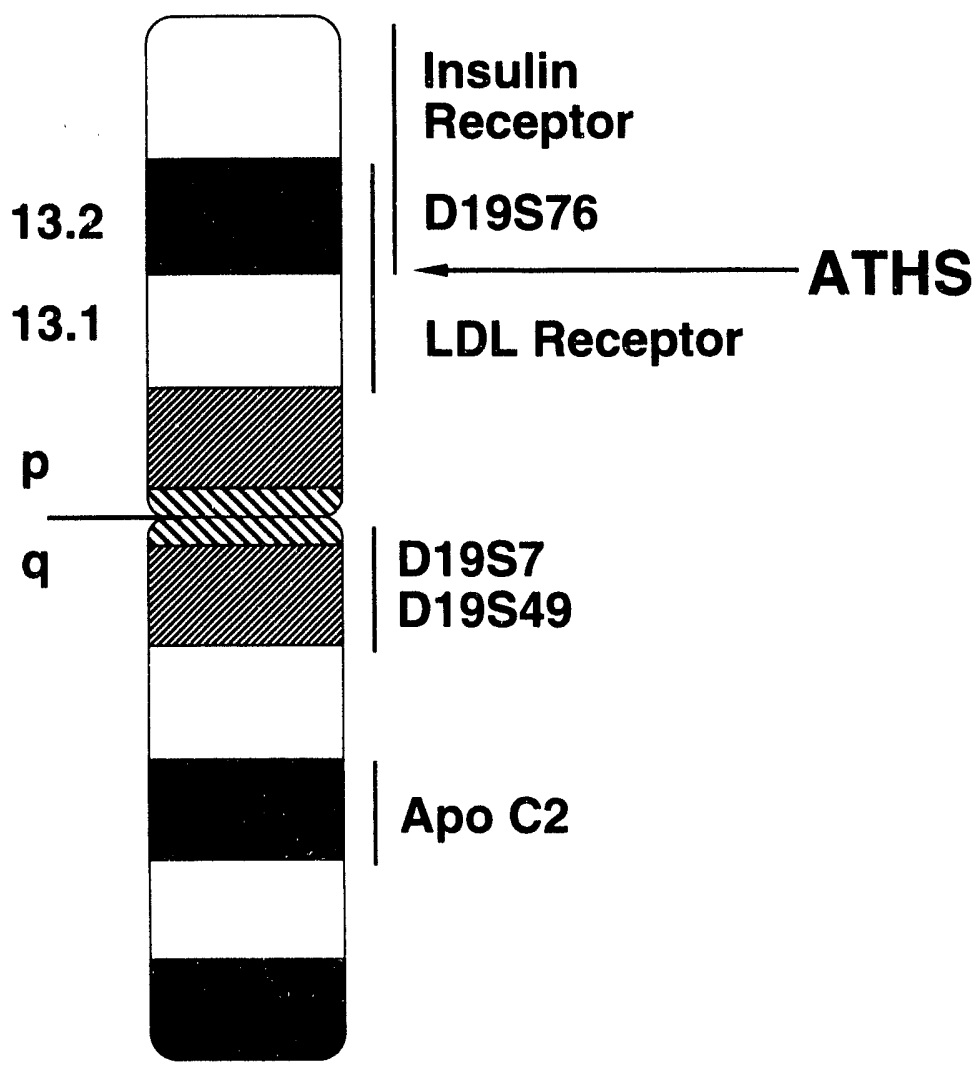

\section{National Institutes of Health}

The success of DOE Biosciences and environmental sciences programs at LBL has depended not only on DOE support but also on complementary $\mathrm{NIH}$-supported research that is closely coupled to these programs. Several critical technologies recently identified as being of high priority for advancement by NIH, specifically molecular medicine, biotechnology, and struc-

tural biology, are all major components of the life sciences program at LBL. These technologies build on the unique facilities and expertise available at $\mathrm{LBL}$, and point toward a growing interaction of DOE and $\mathrm{NIH}$-funded research in pursuing new goals in biology and medicine.

$\mathrm{NIH}$ supports programs on radionuclides, NMR, diagnostic image reconstruction, and radiopharmaceuticals related to advanced instrumentation and disease treatment. Other major $\mathrm{NIH}$-funded programs involve lipoproteins and their relationship to cardiovascular disease, biological structure analysis by electron crystallography to characterize cellmembrane proteins and viruses, and the intracellular molecular structure of DNA and sickle hemoglobin.

NIH applies LBL's unique resources to investigations of the human genome and in carcinogenesis and mutagenesis.

Repair and recombination in yeast and the genetic effect of carcinogens will continue to be major foci. Cell nuclei are studied by circular dichroism and related techniques. The Laboratory's capability in culturing human mammary epithelial cells is used to study breast cancer. 
The National Tritium Labeling Facility conducts research into the labeling of compounds with tritium. LBL also conducts a program on intermediatevoltage biological electron microscopy under NIH sponsorship. NIH also supports research on oxygen radicals and aging, environmental tobacco smoke, soil transport of gas pollutants, arıd ecotoxicology assays.

\section{Department of Defense}

The Army Strategic Defense Command supports neutral-beam research related to magnetic fusion energy. The Air Force Office of Scientific Research supports $x$-ray microscopy and high-brightness $x$-ray studies. The Office of Naval Research supports studies of oxide scales on aluminides, quantum Monte Carlo calculations, thin-film superconductors, zinc cells, and microwave-radiation-stimulated release of drugs. The Navy also sponsors research on efficient lighting for ships and optical properties of the ocean.

The Advanced Research Projects Agency (ARPA) is expanding its support of research in $\mathrm{x}$-ray lithography at the Center for X-Ray Optics (CXRO). The projects supported by ARPA are on the critical path for the development of extreme ultraviolet (EUV) pattern transfer technology. The research is in response to unique opportunities for science and technology at the Advanced Light Source (ALS). Funding for the design of an undulator beamline has already (FY 1991) been received through LBL's Center for XRay Optics. CXRO is also acquiring an advanced direct-write electron beam lithography system with ARPA funding. This e-beam writer, or "nanowriter," will be used to support $x$-ray lithography research through the fabrication of zone plates and $x$-ray masks. Funds for new instrumentation are expected to continue in FY 1994.

ARPA also funds the MAGIC gigabit testbed, a project to combine highspeed wide-area network technology, distributed image storage systems, and high-speed graphics with aerial and satellite images to present a user with a virtual reality that corresponds to what a real observer would see while traveling through the real terrain. LBL is doing the research and development for the high speed distributed image server system that will supply the imagery to the terrain visualization application that provides the real-time view of the landscape. LBL may also receive increased ARPA support for dual-use R\&D for magnetic materials microscopy and for molecular design.

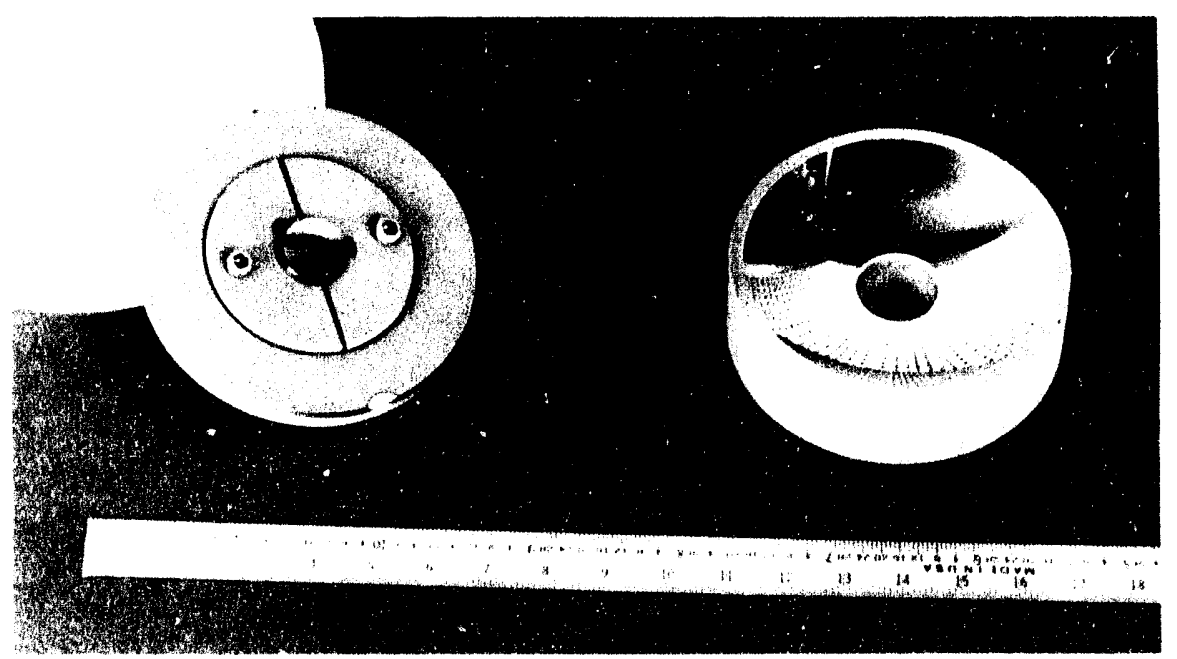

Coated optics with controlled multilayer period variation for $68-\AA$ wavelength operation. The optics were made by AT\&T, coated with DOE support at LBL's Center for $X$ Ray Optics, and experiments in collaboration with AT\&T will be done with DOD support at Brookhaven National Laboratory. These will be demonstration experiments for nano-electronic pattern transfer studies. 
At a site near the Stillwater Marsh in Nevada, test wells were drilled 300 feet deep to study the groundwater. By detailed characterization of the drainage, cost-effective recommendations were made to reduce drainage contaminants. This project is an example of how scientists at $L B L$ are helping federal and state agencies solve problems in soil contamination and resource management.

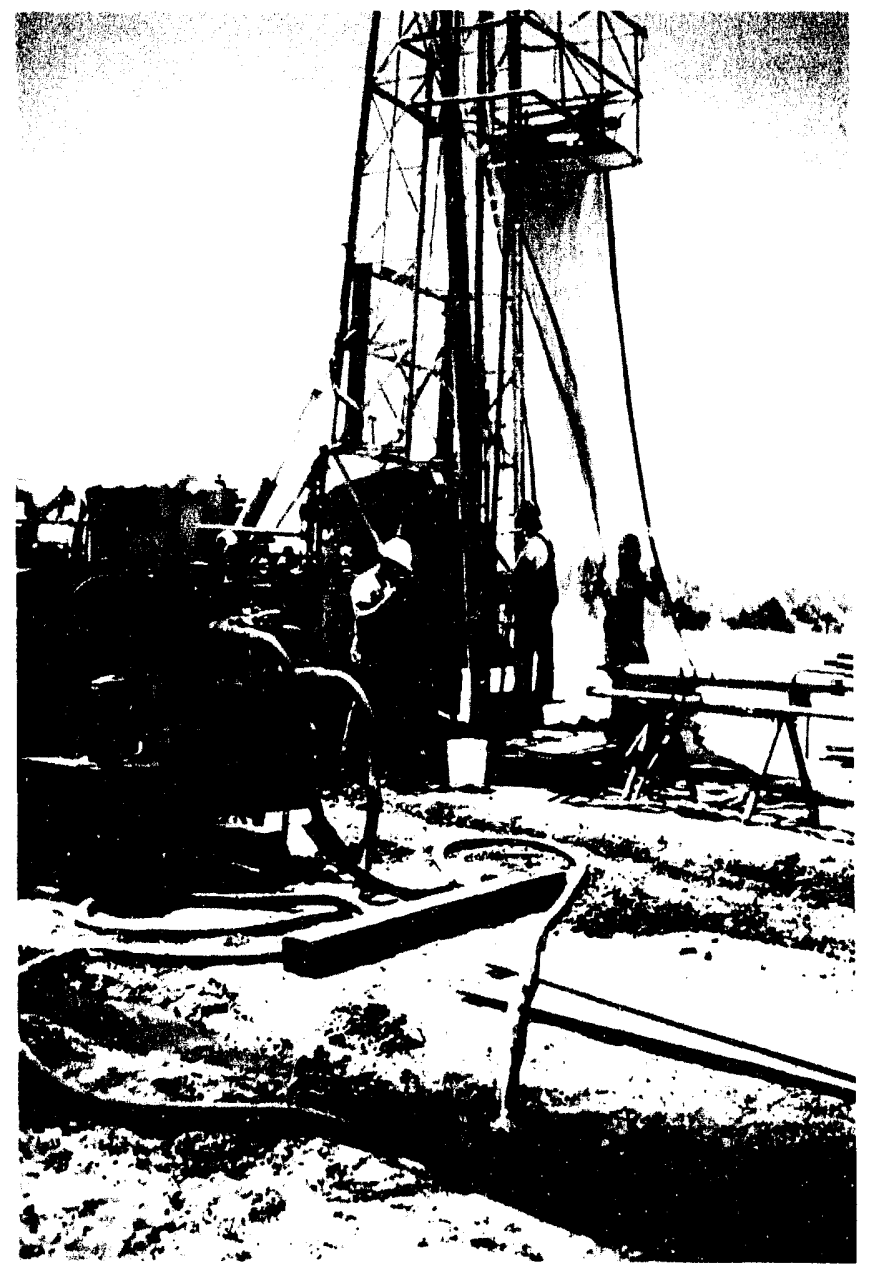

\section{National Aeronautics and Space Administration}

LBL is conducting radiation health and instrumentation-development projects for NASA. In collaboration with Colorado State University, Fort Collins, LBL is recognized as the NASA Specialized Center for Research and Training (NSCORT) in these areas. LBL also collaborates with various NASA groups to investigate the cosmic microwave background radiation and to develop gamma and $\mathrm{x}$-ray detector systems for various space applications, such as for the WIND Spacecraft and Nuclear Astrophysics Explorer. LBL scientists are developing a superconducting magnetic spectrometer for the space station to measure cosmic-ray particles and gamma rays in search of exotic matter. Other ongoing research concerns nitrogen recycling in a Closed Ecological Life Support System for long-term space missions.

The LBL Astrophysics Group has been instrumental in the development of the Differential Microwave Radiometer installed on the Cosmic Background Explorer (COBE) which detected anisotropies in the cosmic microwave background. These anisotropies show the primordial seeds of modern structures such as galaxies, clusters of galaxies, and things of larger scale. These seeds are produced by particle interactions at the creation of spacetime. Data collection and analysis is continuing, to improve statistics and to refine our understanding of the early universe.

\section{Environmental Protection Agency}

LBL conducts research on the hydrogeological transport of contaminant plumes from deep underground injection disposal. In the area of global environmental effects, LBL is characterizing the emissions of energy technologies, improving global energy projections, fostering international awareness of global trends, studying effects of tropical deforestation, and gathering information on the potential effect of global climate change on U.S. natural resources. LBL, with other national laboratories, is working to develop new programs in partnership with EPA that advance national environmental goals.

\section{Department of the Interior}

Laboratory scientists are investigating the geochemistry of selenium and other trace elements at Kesterson Reservoir, which has been a terminus of agricultural drainage water in California's San Joaquin Valley. Continuing collaborative investigations are underway to evaluate remediation techniques for the area's soil. Related research is being conducted at Stillwater Marsh, Nevada.

\section{Agency for International Development}

The Agency for International Development is supporting a multiyear effort in which LBL will perform research in support of improving the efficiency of energy use in developing countries. 


\section{Other Agencies/State and Private}

The Laboratory conducts research for the Electric Power Research Institute (EPRI). Chemistry-related research includes siudies on reducing oxidation and scale formation, on the development of chemical "mimics" of natural enzymes for methane conversion, and on oxygen depletion in compressed-air storage. Another EPRI project is on studies of surface modification with metal plasma techniques.

The Gas Research Institute supports databases on the influence of clays on seismic wave attenuation in reservoir rocks. The California Air Resources Board is sponsoring an analysis of polycyclic aromatic hydrocarbons in indoor air.

LBL's expertise in buildings is recognized by the California Energy Commission and the energy utilities. Much of the support is through the California Institute for Energy Efficiency (CIEE), a joint effort among the California energy utilities, LBL, the University of California, and the California Energy Commission and Public Utilities Commission. CIEE manages a focused research program, with the research carried out by California universities and DOE national laboratories located in the state. CIEE's overall research framework is determined by a research board consisting of high-level executives from the utilities, the University, the Commissions, DOE, the Gas Research Institute, and the Electric Power Research Institute. A Planning Committee with members from these same institutions approves individual research projects within this framework. To LBL researchers, CIEE appears in effect as a WFO agency which evaluates and funds proposals from LBL along with those from other California research institutions. Particular LBL projects funded by CIEE include the study of efficient systems for thermal distribution in buildings, integrated envelope and lighting technolngies, end-use technology performance data, urban landscape modifications to reduce energy use and air pollution, and advanced combustion devices to reduce nitrogen oxide emissions.

Additional utility support is as follows: Southern California Edison supports window studies; Pacific Gas and Electric Company supports a study of end-use energy intensifiers in commercial and residential buildings; and the Sacramento Municipal Utilities District supports energy-savings studies of shade trees and other surfaces.

The LBL-designed Keck 10-m telescope, the world's largest optical instrument, is completed. LBL anticipates funding from UC Santa Cruz/Lick Observatory to perform work in support of the equally large companion Keck II. In the life sciences, research on human lipoprotein function and genetics is supported by the National Dairy Board through Children's Hospital in Oakland. The UC California Tobacco Research Institute supports research on carcinogenesis. The National Cancer Institute supports basic research on how carcinogenesis is initiated by chemicals and radiation. Additional studies are funded to investigate how growth is normal and cancer cells are controlled by their microenvironments. The California Competitive Technology Program is supporting several LBL-industry collaborative R\&D projects in the Center for Advanced Materials, including high-temperature thin-film superconducting device development and selfassembling thin-film sensors. 


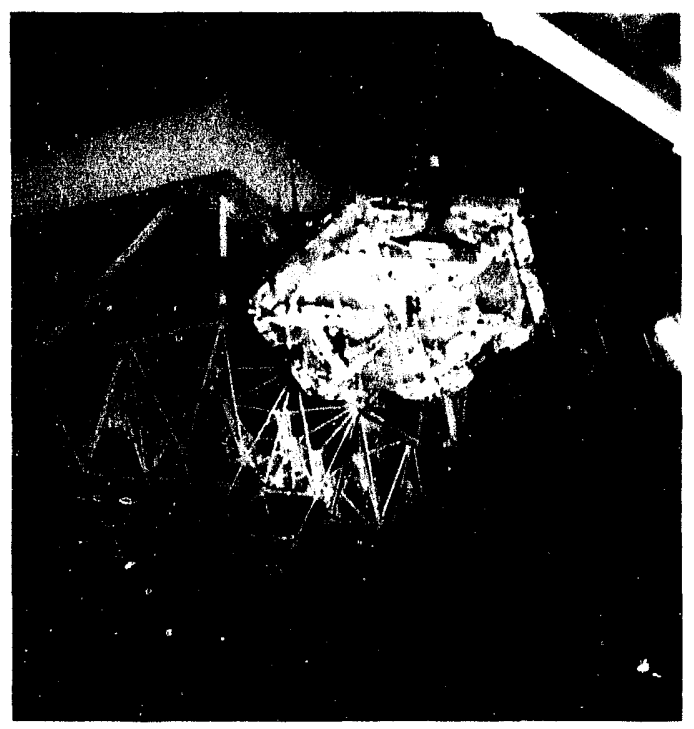

The original concept and proof-of-principal studies for tite Keck Telescope's segmented mirror, which made the world largest teiescope possible, were done at LBL. Here is shown a hexagonal mirror segment lifted by crane for installation, and the first celestial image of spiral galaxy NGC 1232.

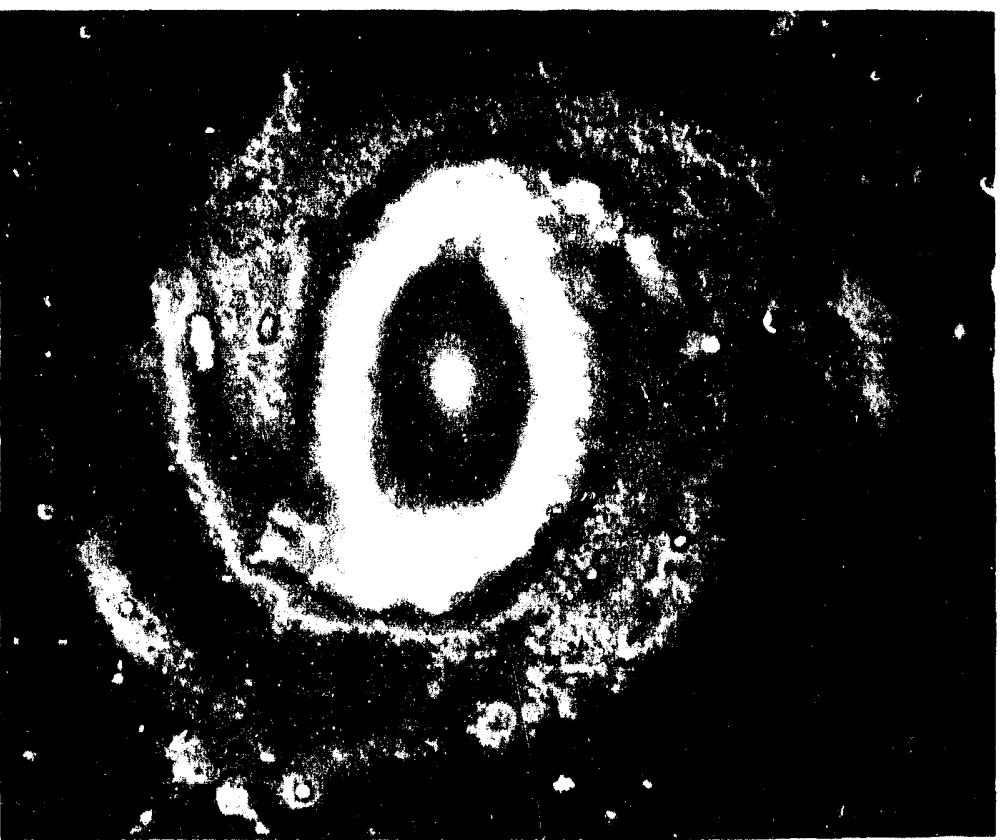

\section{LABORATORY DIRECTED RESEARCH AND DEVELOPMENT}

The Laboratory Directed Research and Development (LDRD) Program was established in 1984 following the issuance of DOE guidance to allocate a portion of LBL's operating budget to explore innovative research opportunities. These allocations are subject to the approval of DOE.

Laboratory Directed Research and Development Program

\begin{tabular}{lcccc}
\hline Category & $\begin{array}{c}\text { FY 1992 } \\
\text { Actual }\end{array}$ & $\begin{array}{c}\text { FY 1993 } \\
\text { Actual }\end{array}$ & $\begin{array}{c}\text { FY 1994 } \\
\text { Projected }\end{array}$ & $\begin{array}{c}\text { FY 1995 } \\
\text { Projected }\end{array}$ \\
\hline Funding (\$M) & 4.9 & 5.1 & 7.6 & 7.8 \\
Projects Approved & 46 & 48 & - & - \\
\hline
\end{tabular}


The LDRD-funded work in synchrotron $x$-ray fluorescence enables researchers to do micro-scale mapping of trace element distributions and correlations in soils. An immediate application is the measurement of heterogeneities of contaminant distributions within soils. Pictured is the micropobe that has been used at Brookhaven National Laboratory.

$L B L$ researchers have successfully synthesized and characterized sizable single crystals of both pure and doped $C_{60}$ including single crystals of $R b_{3}, C_{60}$ and $K_{3} C_{60}$. The magnitude and temperature dependence of the intrinsic crystal resistivity exhibited by the single crystals can be used to distinguish between competing electronic transport and superconductivity models.
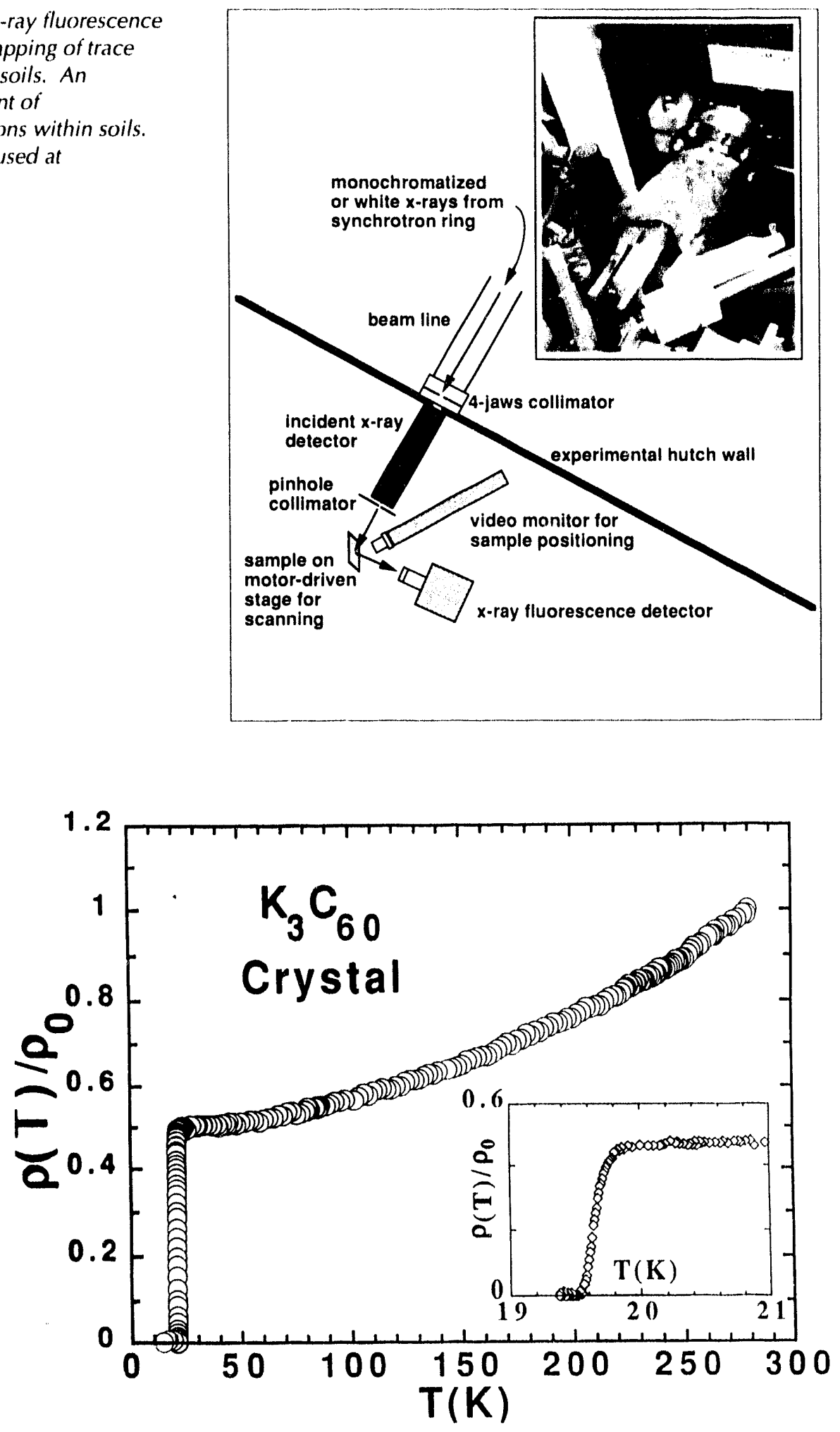
This program contributes to scientific staff capability and vitality through the support of new research programs of merit and potential. Examples of project areas eligible for support include:

- Work in forefront areas of science and technology that enriches Laboratory research and development capabilities;

- Advanced study of new hypotheses, new experiments, and innovative approaches to development of new concepts or knowledge;

- Experiments directed toward proof of principle for initial hypothesis testing or verification; and

- New device studies to explore possible application to instrumentation or experimental facilities.

Recent achievements sponsored by the LDRD program in the Energy Sciences include research in interface and nanostructure materials, novel displacement detectors for seismology, time of flight spectroscopy of rare gases, $x$-ray reduction lithography, novel chemical dynamics studies, studies on fullerenes, new high pressure semiconductor processing, and advanced techniques for electron microscopy. Research achievements in the General Sciences were directed towards prototype tracking and timing detectors, ultraviolet free electron lasers, new types of silicon tracking systems, and new data acquisitions systems. Achievements in the Biosciences areas included analysis of a cell cycle regulatory gene, single DNA molecule chemistry, testing of novel mismatch repair enzymes, $x$-ray crystallographic studies of RNA, and environmental air pollutants and oxidative stress. Achievements in the Resources and Operations areas include studies on neutron detectors for future applications, and studies in high density packaging of high frequency integrated circuits. An Annual Report on the LBL Laboratory Directed Research and Development Program is available from the Office for Planning and Development. Annual planning documents are prepared that indicate program directions and projected resources. 


\title{
6 ENVIRONMENT, SAFETY,
}

\author{
All of our activities will be conducted with full regard for \\ the environment, health, and safety.
}

LBL Vision 2000

\section{ES\&H GOALS AND OBJECTIVES}

It is the policy of the Lawrence Berkeley Laboratory to integrate environment, safety, and health (ES\&H) performance in the conduct of all of its operations to ensure employee and public safety and the protection of the environment. The Laboratory has developed institutional ES\&H goals to guide its integrated ES\&H program. The ES\&H goals are the following:

- LBL will provide employees with a safe workplace.

- LBL will design and operate facilities and research activities to minimize adverse impact on public health and the environment.

- LBL will produce and use only materials that can be disposed of safely and will minimize waste.

- LBL will promptly communicate the known hazards of our activities and the related methods necessary for safety and health protection.

- LBL will use available technology, engineered safeguards, and responsible science to mitigate all significant risks arising from its research and related activities.

The objectives of independent research activities are to ensure the integrity of human health and safety and the environment in which we operate, to maintain a capability that is not currently supported by other Laboratory programs, to provide opportunities for staff development, to build new competencies that could prove useful to future Laboratory and DOE ES\&H programs, and to support the Laboratory's technology transfer mission. 
Underlying these goals is a commitment to ES\&H performance through quality management of the Laboratory's programs and in its conduct of operations. LBL's current efforts respond to the management initiatives of the DOE Office of Energy Research (ER), and directly to the Corrective Action Plan for the Tiger Team Assessment and ER review. The Laboratory has also developed a new environment, safety, and health five-year plan and is developing implementation programs for a DOE Environmental Restoration and Waste Management five-year plan. These efforts include a renewed commitment to ES\&H through self-assessment and an effective Corrective Action Plan.

The Laboratory's ES\&H performance indicators are utilized to improve performance and institute a more quantitative framework for LBL's ES\&H trends and activities. For employee health and safety, representative indicators include the absolute number and rate of injuries (i.e., frequency) and the number of workdays lost and rate of workdays lost (i.e., severity rate). The Laboratory seeks to establish trends of continuing improvement in ES\&H performance for FY 1994 compared with performance in recent years.

Environmental indicators include improving waste management operations and waste minimization, with the goal of managing waste disposal more effectively and efficiently, including significantly reducing the total amount of hazardous waste generated for FY 1994. Waste minimization indicators include the percent of Laboratory office waste recycled and the total number of solid, hazardous, and radioactive waste streams recycled. Performance indicators include: number of waste pick-up requisitions rejected because waste is nonhazardous (waste minimization), number of complaints received by group leader (waste management operations), and number of requisitions received and processed with average turnaround time for each requisition (waste management operations). In addition, the Laboratory is eliminating any excess discharge of heavy metals or toxic chemicals to the sewer system, with no exceedances of standards as the Laboratory's goal.

To facilitate management performance, the Laboratory is developing improved standards for individual employee ES\&H responsibility, calling for formally defined responsibilities for each employee as a goal (100 percent

ES\&H Performance Indicators

\begin{tabular}{|c|c|c|c|c|c|c|c|}
\hline Indicator & 1987 & 1988 & 1989 & 1990 & 1991 & 1992 & $\begin{array}{c}1993 \\
\text { (Projected) }\end{array}$ \\
\hline Injuries & 62 & 50 & 75 & 78 & 101 & 99 & 100 \\
\hline Injury Rate & 2.6 & 2.0 & 3.1 & 3.2 & 4.0 & 3.8 & 3.8 \\
\hline Lost Workdays ${ }^{b}$ & 592 & 442 & 295 & 810 & 1409 & 1516 & 1500 \\
\hline Lost Workday Rate & 25 & 18 & 11.1 & 33 & 55.7 & 60 & 60 \\
\hline Division OSHA Training (\%) & 0 & 0 & 0 & 0 & 66 & 71 & 100 \\
\hline Office Waste Recycled (\%) & n.d. & n.d. & n.d. & n.d. & 80 & 82 & 87 \\
\hline Recycled Waste Streams ${ }^{d}$ & n.d. & n.d. & n.d. & 23 & 26 & 35 & 37 \\
\hline
\end{tabular}

\footnotetext{
"Rate is expressed as number per 100 FTEs, approximately 200,000 person hours of work.

bystem and comprehensiveness of tracking has undergone improvement in recent years.

'Percent of divisions with OSHA-trained safety staff (estimated).

dNumber of solid hazardous, and radioactive waste streams recycled; n.d. = nof determined
} 
confirmed level for 1994). The Laboratory also has the goal of providing Occupational Safety and Health Act (OSHA)-trained expertise in each division.

From 1987 to present the first quarter report of injuries and illnesses with associated injury rates and lost workdays for LBL indicates a $65 \%$ increase (from 592 to 1500 incidents: a plus 908 incidents). Possible contributors to this increase are:

- DOE requires that all recordable injuries be documented and recorded. In the past this policy was not clear and many first aid incidents did not get recorded. All first aid injuries are now required to go to medical.

- In 1992 the Occupational Safety Group added seven safety professionals. These additions increased the reporting, the documentation, and the administration of reporting accident/injury type incidents.

- In an effort to better identify causal factors and corrective action, the Laboratory has established and implemented an accident investigation training program to reduce potential for recurrence of similar type accidents. Subsequently this will assist in reducing the frequency of injuries. This type of program was not in place prior to 1993.

The accident investigation training program will require some time to be evaluated in its effectiveness in reducing the number of recordable injuries and illnesses at LBL.

This Environment, Safety, and Health Management subsection provides a framework for identifying and integrating long-range programmatic activities with ES\&H resource plans. The following subsections identify current ES\&H operating conditions and trends, management arrangements, and management strategies and initiatives. This framework supports the Office of Energy Research efforts to effectively plan and assess safety and health support, facilities infrastructure needs, and long-range environmental compliance programs, such as National Environmental Policy Act (NEPA) compliance and site restoration programs.

\section{CURRENT CONDITIONS}

\section{Programmatic Directions and Potential Hazards}

As indicated in Section 5, LBL's scientific and technical programs primarily support DOE's Office of Energy Research (62\%). The multiprogram character includes programs in Basic Energy Sciences, Nuclear Physics, High Energy Physics, and Health and Environmental Research. Energy Efficiency and Renewable Energy (6\%) supports studies in building energy conservation, energy storage, and solar and geothermal energy. Other DOEsponsored programs $(16 \%)$ include research supported by the Superconducting Super Collider Laboratory, Civilian Radioactive Waste Management, and Fossil Energy. Work for other agencies and institutions (16\%) is primarily for the National Institutes of Health, Department of Defense, states, and private industry.

LBL's potential ES\&H risks are characteristic of accelerator operations, shops, and a diversity of laboratories for chemical, biological, materials science, and technology development, as well as other facility support operations. Hazards arise from both radiological and nonradiological activities. Radiation protection for workers and the public is required for 
accelerators, $x$-ray units, sealed sources, and radioisotope use. The nonradiological hazards include electrical systems, sources of ignition and combustible materials, rotating and reciprocating machinery, hoisting and rigging operations, lasers, chemicals, moving vehicles, construction activities, and natural pinenomena such as storms and earthquakes.

LBL monitors the levels of chemicals and radioactivity discharged from operations at the Laboratory, and evaluates their impacts on the environment and to the public health. Radiological operations include particle accelerators, gamma irradiators, laboratories conducting research using radionuclides and radiopharmaceuticals, and the National Tritium Labeling Facility. Nonradiological sources and discharges include chemicals from research operations, fabrication shops, automotive shops, paint shops, water treatment facilities, and the Hazardous Waste Handling Facility. Current trends in Laboratory activity indicate environmental and safety hazards that must be mitigated:

- Chemistry and Materials Research. Programs in materials and chemical research, including new hazardous materials management requirements, create demands on many ES\&H programs. Examples include managing chemical acquisition and inventories, chemical monitoring systems, fume hood monitoring, laser safety training, respiratory protection programs, and hazards communications. The Advanced Light Source, a controlled facility, will greatly expand the use of $x$-rays on site, and will be operated with complete ES\&H systems and management protocols in place.

- Biological Research. LBL biological research programs have grown recently. LBL's biological hazards control program continues to be expanded. Biological research programs often utilize radiolabeled materials, and the delivery of low-level radioisotopes has increased in the last few years. The radioactive and mixed wastes derived from these operations present a special challenge because of the restrictions on their disposal.

- Radiobiology Experimental Programs. LBL radiological research at the Bevalac has closed, but the use of isotopes is expected to continue in many applications, including the new Biomedical Isotope Facility and a proposed National Tracer Facility.

- Construction. LBL's construction activity has increased during the past decade, resulting in potential additional accident risk. ES\&H oversight for construction contractors and tradespersons has become increasingly important, including, but not limited to, the need to ensure the stability of excavations and management of drainage systems.

- Nuclear Physics and Nuclear Chemistry. The nuclear physics experimental programs at the Bevalac were curtailed in FY 1993. However, the 88-Inch Cyclotron anticipates expanded research with the Gammasphere detector and supports a more diversified scientific program. In addition, planning is under way for an IsoSpin Laboratory that will utilize radioactive isotopes.

Increased resources have been provided to serve research program areas with potential hazards, such as those employing toxic chemicals, lasers, new $x$-ray sources and beamlines, and radiolabeled compounds. Space for staff and equipment must be provided to meet service demands and logistics needs. Reporting functions are being formalized to optimize use of staff. 
Automated systems to track chemicals from procurement to disposal are under development.

\section{Response to Regulatory Requirements}

LBL ES\&H polices and procedures are being developed to iully comply with existing, proposed, and anticipated DOE Orders, federal laws and regulations, and applicable state and local regulations. For maintaining and improving air quality, LBL addresses requirements of the Feder.al Clean Air Act and the California Clean Air Act and obtains permits consistent with Environmental Protection Agency (EPA) policies, including National Emission Standards for Hazardous Air Pollutants (NESHAPs), and those of the Bay Area Air Quality Management District. The Laboratory conducts its operations and monitors its effluents consistent with its National Pollution Discharge Elimination System (NPDES) permit and its East Bay Municipal Utility District Wastewater Discharge permits. For protecting surface water and groundwater, LBL conducts programs consistent with the Federal Clean Water Act, the Safe Drinking Water Act, and the California Oil Pollution Control Act. In addition, the Laboratory complies with the requirements of the California Porter-Cologne Water Quality Act, including programs permitted through the San Francisco Bay Regional Water Quality Control Board and the East Bay Municipal Utility District. LBL further protects and monitors the environment from radiation releases as directed by the Atomic Energy Act and monitors wastes through compliance with the Resource Conservation and Recovery Act (RCRA) and the Comprehensive Environmental Response, Compensation and Liability Act (CERCLA).

In addition, LBL policies, procedures, and operations must fully comply with the Toxic Substances Control Act and the Federal Insecticide, Fungicide and Rodenticide Act to control the storage and disposal of hazardous materials, including polychlorinated biphenyls. Consistent with the Hazardous Materials Transportation Act, LBL provides complete manifests for shipped wastes and packages and labels materials to comply with Department of Transportation Regulations.

LBL safety and health programs also address requirements of OSHA, DOE Orders, and a broad range of industry standards on occupational protection. The Laboratory's Conduct of Operations and Quality Assurance (QA) plans are addressing appropriate guidelines of new DOE Orders. QA for planning, monitoring, testing, controlling, and reporting is being directed to meet the objectives of DOE General Design Criteria, reporting, and environmental protection and safety standards, as well as methods for analysis established by EPA.

LBL projects and new proposals are reviewed for conformance with NEPA and the California Environmental Quality ACt (CEQA), including the goals to protect the environment and provide safe and healthful surroundings. The Laboratory provides environmental reviews and performs assessments consistent with NEPA and CEQA guidelines. The Laboratory incorporates procedures and documentation responsive to the Endangered Species Act, the Federal Fish and Wildlife Coordination ACt, and the National Historic Preservation Act. LBL cooperates with the State of California Resources Agency, including the Department of Fish and Game, to identify and protect threatened or endangered species, and the Office of Historic Preservation to identify and protect cultural resources. 
LBL alsu coordinates ES\&H activities with the cities of Oakland and Berkeley, including environmental activities and mutual-aid fire-protection programs and traffic management. Examples of this coordination include (1) a hill-area fire-safety program with the University and Berkeley fire departments, (2) an LBL traffic and parking management plan to discourage singleoccupant vehicles, (3) a compliance program to provide the City of Berkeley with a Hazardous Material Inventory, and (4) a monitoring program for underground storage tanks. The Laboratory's construction program and facilities planning are conducted within the framework of a Long Range Development Plan approved by The Regents of UC and plans approved by DOE.

\section{Review Activities and Accomplishments}

LBL has been active in its self-assessment program, the application of performance indicators, and in the integration planning for ES\&H resources and operations. The recent ER Tiger Team follow-up reviewed the commitment of the Laboratory Director and the improvement of ES\&H conditions at LBL. The ER team found examples of notable practices:

- The process of preparing LBL plans such as the Institutional Plan has had a positive effect on providing visibility of ES\&H activities and promoting broad discussion of goals, expectations, and plans for the Laboratory divisions.

- LBL's Performance Indicator Program shows clear evidence of commitment and effort to identify, monitor, and measure performance.

- The Division Self-Assessment Plan, such as in the Accelerator and Fusion Research Division, is an excellent example for implementation of the self-assessment process.

- LBL's Operations Office has demonstrated awareness of the use and value of occurrence reporting and the desire to improve performance through risk identification and the re-emphasis of safety practices.

- The notebook approach to Laboratory activities and projects shows an innovative approach to address the management findings of the Tiger Team Assessment.

The Laboratory continues to undertake institution-wide efforts to enhance safety and protect the environment. Examples of other efforts in FY 1993 serve to highlight the steps the Laboratory is taking to develop a meritorious ES\&H program:

- LBL has finalized and implemented compliance guidelines and initiated a new NEPA/CEQA training program, and fully implements its NEPA/CEQA trackirig system. All Field Task Proposals, Work For Others proposals, CRADAs, grant proposals, Laboratory Directed Research and Development proposals, and construction projects include review information for NEPA/CEQA compliance consistent with LBL's compliance program.

- LBL has served as a leading Laboratory in the development of a riskbased prioritization system for the development of five-year safety and health plans. LBL is serving as a testbed for prioritization plans and has led workshops for contractor and field personnel. 
- Comprehensive cleanup of laboratories and support facilities resulted in recycling of more than 727,000 pounds of metals and other materials to the local salvage industry, more than double the previous year's average. In addition, LBL has instituted a comprehensive recycling program in which all the Laboratory's nonhazardous waste is sorted for recycling, resulting in $80 \%$ of the waste being recycled and only $20 \%$ going to landfills. In addition, LBL has reduced the amount of waste sent to landfills by two-thirds.

- Advances in high-voltage safety in electrophoresis have been made through special power supplies, shielded connectors, ground fault interrupters, and interlocks. These advances, recognized as a noteworthy practice by the 1991 Tiger Team, are being transferred to manufacturers.

- Chlorofluorocarbon recycling equipment has been acquired that allows for repair and reclamation of refrigeration equipment without freon releases. Special protective suits and gas monitoring equipment have been acquired, and procedures for confined space entry and lockout tagout have been upgraded.

- Comprehensive fire and electrical inspections have been implemented. As a result, a number of changes, such as additional fire doors, better fire alarms, and improvements in the type and availability of fire extinguishers have been made as initial steps in a program to upgrade fire and electrical safety.

- Materials Safety Data Sheets, other hazard information, and chemical inventory data are being made accessible to LBL's distributed computer network, enabling this important information to be readily available across the LBL site.

- LBL's printing plant converted to use of recycled paper and vegetablebased ink where possible.

There have been accomplishments in the particular area of ES\&H training.

- Implementation of a new training database to track all training requirements, including environment, safety, and health. The database, with report generation capabilities, is accessible Lab-wide.

- Production of a hazardous waste training video entitled "Environmental Compliance: Hazardous Waste and Radioactive/Mixed Waste." This video, coupled with a guidebook and written exam, enables divisions to complete required generator training at their convenience.

- Development and implementation of a four-hour chemical safety training for chemical users to comply with OSHA laboratory safety and hazard communications requirements. Course is given by division to meet divisional needs.

- Production of an educational video entitled "Radiation Dosimetry" to inform all those on LBL's personal dosimetry program the proper use of persorial dosimeters and requirements for internal dosimetry.

- Publication of health and safety handbooks for new employees, participating visitors, contractors, and guests to inform them of the hazards that may be encountered on-site, available resources and proper emergency response. 


\section{Environment, Safety, and Health Management}

These examples are indicative of the developing momentum that is strengthening LBL's ES\&H performance and compliance. A broad range of changes is now being advanced, as indicated in the Management Strategies and ES\&H Plans and Initiatives sections below.

\section{ES\&H POLICIES, ORGANIZATION, AND MANAGEMENT}

\section{Policies and Organizational Responsibilities}

$D O E, U C$, and LBL have strengthened policies and resources to address DOE ES\&H initiatives. The Office of Energy Research (ER) has landlord responsibility for $L B L$. ER Headquarters provides oversight and direction through its line programs and has established the Office of Assessment and Support, a staff organization with ES\&H expertise to assist ER line programs. $\mathrm{DOE} / \mathrm{SF}$ conducts oversight and communicates programmatic, budgetary, and scheduling guidance for ES\&H activities at LBL. Lead responsibility for implementation of DOE/SF's ES\&H activities at LBL lies with the Site Manager at LBL.

LBL addresses ES\&H planning and operating requirements, including the implementation of DOE Orders, guidelines, and applicable Federal, state, and local ordinances through defined lines of management responsibility. The Regents of the University of California, through the Office of the President, are responsible for corporate policy and contract approval. Within the Office of the President, the Office of the Senior Vice President for Administration has corporate support offices for Long Range Development and Environmental Planning, Hazardous Materials Planning, Environmental Health and Safety, and Environmental Planning for compliance with CEQA. The Senior Vice President for Academic Affairs and the Special Assistant for Laboratory Affairs provide program management support for Laboratory activities and work closely with the administrative units of the University. In recognition of the strengthened requirements for University oversight of ES\&H activities at its laboratories, the responsibilities of the Senior Vice President for Administration have been expanded. A new office to carry out specific oversight activities for ES\&H and a new President's Council on the national laboratories has been established.

The LBL Director is responsible for the establishment and administration of LBL ES\&H policies. The Director is accountable for the ES\&H requirements placed upon the University of California as a consequence of its management contract with DOE. The Director has assigned responsibilities and authorities necessary to implement and oversee the Laboratory's policies regarding ES\&H to Associate Laboratory Directors and Division Directors.

Management policies, procedures, and responsibilities for supervisors and employees are formally communicated in the Regulations and Procedures Manual (PUB-201) and the Health and Safety Manual (PUB-3000), and separate technical documents and safety procedures address specific operations. The Occupational Safety Department is leading the major effort to rewrite the Laboratory's Health and Safety Manual to incorporate all new safety policies. Applicable rules and procedures extend to all Laboratory operations. It is the responsibility of supervisors to ensure that employees are familiar with these publications and to apply the rules and regulations in 
planning and carrying out their work. Compliance with ES\&H policies and procedures is implemented, enforced, and ensured through line organization and support and control organizations:

- Division Directors are responsible for ensuring that the Laboratory's environmental, safety, health, and emergency preparedness policies are followed by employees within their own divisions.

- The Associate Laboratory Director for Operations is responsible for the oversight and management of the technical support resources at the Laboratory, including ES\&H operational support resources, and provides corrective action, self-assessment planning and reporting, and quality assurance to ensure that the environmental, health, and safety management systems and procedures of the Laboratory are implemented by the divisions.

- The Director of the Environment, Health \& Safety (EH\&S) Division is responsible for supporting and enforcing the implementation of Laboratory-wide environmental, safety, and health programs. The Division provides technical support and technical audits to LBL operating programs for compliance with applicable ES\&H standards, with line management being responsible for the actual implementation.

- The Associate Laboratory Director of the Office for Planning and Development integrates ES\&H plans in LBL site-wide planning processes, including the Institutional Plan and Site Development Plan, and manages the LBL NEPA and CEQA programs for LBL plans and proposed programs.

\section{Management Strategies}

LBL has developed focused strategies directed toward mitigating underlying ES\&H problems and issues. These strategies are part of the Corrective Action Plan to institutionalize ES\&H throughout the Laboratory, build ES\&H resources for compliance with the underlying regulatory framework, and strengthen the relationship between DOE and LBL:

- Develop and Implement ES\&H Management Framework. Management goals for addressing LBL's ES\&H are defined in this Institutional Plan and indicate the Laboratory's commitment to ES\&H. LBL has defined management responsibilities, maintains a well-understood system for prioritizing needs, and is a!locating resources in the framework of long-range ES\&H planning.

- Formalize ES\&H Roles, Responsibilities, and Accountability. At every working level LBL is implementing policies to ensure that all employees know what is expected of them. LBL managers are being evaluated on how well they implement ES\&H policies. A reassessment of organizational responsibilities is being performed to eliminate unnecessary duplication of function, to clarify expectations, and to make sure that no important functions are omitted.

- Strengthen the Central ES\&H Organization. Closely related to the development of an improved ES\&H management strategy is an enhancement of the resources available to LBL's ES\&H organization. Increased resources, both in terms of numbers of qualified ES\&H personnel (nearly doubled since 1991), the range of expertise avail- 
able, and adequate space and equipment, will be essential to correct existing deficiencies and to assist line management in maintaining compliance.

- Multi-Level Self-Assessment Program. A rigorous self-assessment program is the responsibility of the Laboratory and its divisions. The program has been implemented in accordance with the Office of Energy Research Self-Assessment Program plan. LBL's program serves as an important resource to address root causes. Self-assessment is a cornerstone of LBL's efforts to sustain high levels of ES\&H performance.

- Comprehensive ES\&H Training and Communication. More centralized responsibility has been established for coordination of LBL's training activities; existing training activities will be defined and described. The training program will reach all levels of the Laboratory, provide effective targeted material for the different job classifications, and include follow-ups to assess the effectiveness of the training.

- Augment Management Systems. Existing management systems have been augmented to respond effectively to ES\&H needs. ES\&H needs are explicitly included in all LBL long-range planning and programmatic budget-planning processes, and prioritization methodologies are used to ensure that resources are applied to activities of greatest importance.

- Strengthen Interactions with DOE. LBL and DOE have implemented an action plan to strengthen programmatic resources for ES\&H, clarify ER management responsibilities, define lines of communication among $E R$ and DOE field offices and LBL, and institutionalize appropriate $\mathrm{DOE} / \mathrm{SF}$ oversight roles in ES\&H-related activities.

Examples of how LBL management strategies have already been applied are among the following:

- The EH\&S Division has doubled its staff since FY 1991. This planred increase was implemented to adequately respond to ES\&H requirements identified in the 1991 Tiger Team Assessment and in ongoing LBL self-assessments. The new EH\&S Division staff represents a wide spectrum of ES\&H professional disciplines and of ethnic and social backgrounds, bringing a new vitality in addressing the ES\&H needs of the Laboratory.

- Two EH\&S Division student programs are under way. Thirteen students worked from June to September and were mentored by EH\&S Division scientists and engineers. The students were encouraged to attend training in health and safety, government compliance requirements, administrative methods, and computer science. At the end of the summer session, scientific and engineering mentors formally evaluated each student's training and contributions to the EH\&S Division projects and programs.

- Pursuant to OSHA's new Bloodborne Pathogen Standard, the Industrial Hygiene Group is implementing a new Blood Safety Program at LBL. The Standard requires that LBL identify and train all workers who could potentially be exposed to blood and "other potentially infectious materials" while carrying out their assigned job duties. Other program elements include a written Exposure Plan, a Hepatitis-B Vaccination Program, and implementation of control measures and procedures. 
- A Confined Spaces Safety Program has been designed, written, and implemented in response to a Category 11 requirement of the Tiger Team Corrective Action Plan. It is intended to increase awareness of and safety conduct in the Laboratory's confined spaces. The Industrial Hygiene Group is training LBL staff in the recognition of confined spaces, their hazards, permitting procedures, protective equipment and ventilation, and atmospheric testing techniques.

- An Asbestos Management Program is designed to address all aspects of asbestos hazard control; the Pian will include training requirements, hazard identification protocols, air and bulk sampling criteria, contractor specifications for asbestos removal, and specifications for use by construction and facilities personnel to safely maintain the condition of asbestos-containing building materials.

- The Respiratory Protection Program was completed and implemented in February 1992. It establishes procedures and requirements necessary to meet Federal OSHA and ANSI regulations for the use of respiratory protection equipment. The Respiratory Protection Program includes guidelines for training, selection and fitting, and safe use and maintenance of respiration protection equipment.

\section{ES\&H PLANS AND INITIATIVES}

To promote integration of strategic and program planning, the Laboratory maintains a Comprehensive Planning Calendar that defines annual planning requirements and provides a schedule that identifies necessary information exchange and preparation responsibilities. This process defines the responsibilities and information requirements intended to promote the integration of environmental, safety, and health concerns into institutional and program planning elements. Specific working groups strengthen communication, although each planning activity and product is the responsibility of individuals held accountable for the quality and accuracy of the plans. Examples of planning products include those for program plans and for ES\&H support functions:

- ES\&H planning and initiatives. The Environment, Health \& Safety Division (EH\&S) has realigned its mission in service to the broader mission of the Laboratory. To this end, the EH\&S Division effectively manages environment, health, and safety in a way that minimizes interference with new or ongoing scientific research. Creating value for the economy and contributing to the community through partnerships with industry is another part of the Laboratory mission. The EH\&S Division is developing creative solutions for environment, safety, and health problems that can be transferred into the private sector for broader application.

- ES\&H management and operations planning. LBL manages and coordinates its ES\&H programs through strategic plans that define activities, source needs, staffing, and regulating responsibilities. These plans include, as examples, the Tiger Team Assessment Corrective Action Plan, the Safety and Health Five-Year Plan, the Self-Assessment Implementation Plan, NEPA programs planning, and waste minimization plans (see below). In addition, other examples are the Environmental Protection Implementation Plan, the Environmental Monitoring Plan, and the Groundwater Protection Management Plan. 
- LBL emergency preparedness and response planning includes maintaining and updating a Master Emergency Plan, Building and Facility Emergency Plans, and individual equipment emergency plans. $L B L$ is revising and strengthening these plans and implementation programs to ensure that resources and trained staff are available to address all credible emergencies.

- Research program planning. As an example of these plans, early in the formulation of the Induction Linac Systems Experiments (see Section 4), the underlying FS\&H criteria for this facility were established, as documented in a Conceptual Design Report. The safety systems and procedures for this facility are being designed to meet all standards for the expected occupancy. Safety management operations are integrated with pregram plans and are included in cost estimates. The NEPA document preparation schedule and the requirements for Operational Safety Procedures were also planned from the outset. The Human Genome Laboratory underwent a similar review and documentation process.

- Multiprogram Energy Laboratory Facilities Support program planning. LBL's objectives for the MEL-FS program are primarily directed toward ES\&H needs, and the strategic elements of this program are set forth in Section 9. The process and justification, including Corrective Action Plan needs in response to Tiger Team Assessment, are developed in integrated and systematic procedures identified in the Comprehensive Planning Calendar and documented through ES\&H criteria identified in DOE's Capital Asset Management Program. General Plant Projects and General Purpose Equipment planning are also integrated with ES\&H needs (see also Section 9).

- Site development planning. The Site Development Plan integrates ES\&H objectives and needs in all facilities-related programmatic building support projects. Site planning goals, existing conditions, and planning analysis integrate ES\&H planning information, including NEPA and CEQA requirements. Specific references are made to environmental monitoring, environmental impact studies, and the health and safety of facilities. To provide adequate ES\&H support facilities, two general-purpose building initiatives are proposed within the period covered by this plan. Current Laboratory support service facilities are inadequate and inefficient because of obsolete design and substandard construction.

\section{Corrective Action Plan}

LBL's Tiger Team Assessment Corrective Action Plan, completed in September 1991, addresses the findings and concerns of the Tiger Team as well as the 1989 Technical Safety Appraisal. The Laboratory and DOE/SF developed 409 tasks with subsidiary milestones to correct the findings and concerns and eliminate the underlying root causes. These root causes addressed the need for:

- Greater formality of operations and effective verification of the accomplishment of environmental, safety, and health requirements;

- More effectively addressing the challenge of the demands of environment, safety, and health requirements and the urgency of incorporating these demands into LBL operations; and

- Providing DOE program direction and oversight that places adequate emphasis on environmental, safety, and health requirements. 
LBL has made significant progress on the Corrective Action Plan. Over 52\% of the findings have been completed with the completion of $75 \%$ of the corrective actions. All Category $\|$ OSHA findings have been corrected or reduced to lower levels. $L B L$ is continuing to allocate significant resources to the Corrective Action Plan effort. Complete implementation of the Corrective Action Plan also requires the support of the Office of Energy Research and Office of Environmental Restoration and Waste Management as well as additional resources for LBL.

In February 1993, a Tiger Team Follow-Up Review was held at LBL to evaluate LBL's progress on the Corrective Action Plan. Although the Laboratory has not yet received the final report on this review, the draft report was generally positive. The Laboratory has already begun addressing some problems that were identified during the review.

ES\&H Program and Corrective Action Plan Resource Requirements (\$M)

\begin{tabular}{lllllllll}
\hline Category & 1992 & 1993 & 1994 & 1995 & 1996 & 1997 & 1998 & 1999 \\
\hline
\end{tabular}

Corrective Action Plan

Office of Energy Research

\begin{tabular}{lllllllll} 
Operating & 0.2 & 0.8 & 0.7 & 0.0 & 0.0 & 0.0 & 0.0 & 0.0 \\
GPP & 0.0 & 1.7 & 0.1 & 0.0 & 0.0 & 0.0 & 0.0 & 0.0 \\
GPE & 0.1 & 0.0 & 0.0 & 0.0 & 0.0 & 0.0 & 0.0 & 0.0 \\
$\quad$ MEL-FS & 1.2 & 2.2 & 4.0 & 6.0 & 4.0 & 0.0 & 0.0 & 0.0 \\
ERWM & 2.1 & 2.2 & 0.0 & 0.0 & 0.0 & 0.0 & 0.0 & 0.0 \\
Laboratory Overhead & 3.1 & 2.0 & 1.3 & 0.1 & 0.0 & 0.0 & 0.0 & 0.0 \\
$\begin{array}{l}\text { Total Corrective } \\
\quad \text { Action Plan }\end{array}$ & $6.8^{\mathrm{a}}$ & 8.9 & 6.2 & 6.1 & 4.0 & 0.0 & 0.0 & 0.0 \\
\hline
\end{tabular}

Includes data from ERWM table, below.

\section{Environment, Safety, and Health Five-Year Plan}

The Laboratory has developed a prioritized Five-Year Plan for Environmental, Safety, and Health Activities that includes the existing core program of environment, safety, and health services and activities, additional basic support, and specific projects needed to fully meet all LBL and DOE environmental, safety, and health goals. LBL planning has contributed to the development of the ER prioritization system to allocate and rank necessary activities based on quantitative risk reduction criteria.

LBL planning projections indicate that budgeted resources, including those indicated in the Resource Projections (Section 11), will not be sufficient to implement all elements of the planned program without further research program growth or curtailment of other essential Laboratory services. In response to requirements of the Corrective Action Plan and to emphasize safety and health issues, LBL has significantly increased its core environment, safety, and health programs since 1991. Increases in capital resources described in Section 9 and in noncapital resources are also planned. 
Five-Year Plan Projections

\begin{tabular}{llllllllll}
\hline Category & 1992 & 1993 & 1994 & 1995 & 1996 & 1997 & 1998 & 1999 \\
\hline Operating & 13.9 & 18.7 & 23.5 & 27.1 & 28.3 & 28.8 & 29.4 & 30.6 \\
\hline
\end{tabular}

\section{Quality Assurance and Self-Assessment Programs}

LBL has a strong tradition of research productivity and quality, maintaining a commitment to the success of DOE's mission and its research program. The Laboratory is committed to continuous improvement in program performance, environment and safety management, and to the execution of best business practices. LBL has developed a strategy that calls for programs to improve performance based on nationally recognized criteria and is working with DOE on program implementation.

The Laboratory is responsible for maintaining the infrastructure for effective financial and administrative performance. LBL is committed to best business practices and continuous improvement in all areas of administration, including human resource development and training, finance and contractual relations, materials management and procurement, facilities maintenance and engineering, and other management and planning practices. The University and the Laboratory emphasize the application of performance measures. LBL is committed to working with DOE as a partner for continuous performance improvement for national research institutions.

The LBL Operating and Assurance Program (OAP) Plan is the institutional document that specifies the Quality Assurance requirements for the Laboratory. LBL's OAP was developed using the guidance provided in the Implementation Guide for QA Programs for Basic and Applied Research (DOE-ER-STD 6000-92). LBL management distinguishes between activities associated with managing Laboratory resources (facilities and infrastructure) that support research, and activities associated with actual performance of research. In both cases, the extent and detail of the management systems are commensurate with the scale, cost, complexity, and hazards of the work being performed. The requirements specified by the OAP are intended to meet the requirements of DOE Order 5700.6C, Quality Assurance. The Program also contains management system elements of other DOE Orders, where appropriate, and is meant to integrate those elements into the overall LBL approach to Laboratory management.

Through the OAP the Laboratory requires the preparation, use, and maintenance of LBL Function, Facility, and Project "Notebooks" as the primary planning and implementing documents for the OAP. LBL Notebooks establish, describe, and/or reference requirements, responsibilities, and methodologies necessary to perform given activities. They also serve as a repository for documenting such performance.

Not all items, processes, activities, and services have the same effect on health and safety, reliability, environmental protection, or programmatic objectives. Therefore, LBL uses a graded approach to determine the applicability of the OAP requirements to specific Laboratory activities and the rigor with which they should be applied. The objective of LBL.'s graded 
approach is to assist in applying management systems that are commensurate with the scale, cost, complexity, and hazards of the work being performed. LBL line managers are responsible for identifying the activities that are subject to these requirements, and for carrying out an analysis how the OAP requirements are applied.

LBL has implemented its Self-Assessment Program, which provides a formal process for assuring quality and regulatory compliance in all facets of Laboratory operations. It is a continual process of information gathering to determine whether the procedures designed to achieve quality performance are being followed adequately and, if not, what corrective actions are appropriate. It ensures accountability, enables trend analyscs, and improves communication of ES\&H information to line management.

In the LBL program, each division and office is responsible for implementing its individual program, with oversight provided by the Environment, Health \& Safety Division and the Office of Assessment and Assurance. Divisions/offices conduct self-appraisals annually to evaluate their performance in following ES\&H, quality assurance, conduct of operations, and other procedures. The EH\&S Division conducts triennial functional appraisals of division/office compliance with ES\&H procedures. The Office of Assessment and Assurance coordinates triennial internal appraisals by the Safety Review Committee of selective line management activities to evaluate the effectiveness of divisions/offices in ensuring that ES\&H, quality issurance, conduct of operations, and other program goals are being met. The LBL Self-Assessment Program has been nominated for DOE Best Management Practices recognition.

\section{Waste Minimization Plan}

LBL.'s waste minimization program is an organized, comprehensive, and continual effort to systematically reduce hazardous, radioactive, and mixed waste generation. The DOE Waste Minimization and Pollution Prevention Awareness Program and California Senate Bill 14 HazWaste Source Reduction and Management Review are designed to eliminate or minimize pollutant releases to all environmental media from all aspects of the site's operations. These efforts offer increased protection of public health and the environment. They will yield the following additional benefits:

- reduce waste management and compliance costs

- reduce resource usage

- reduce or eliminate inventories and releases of hazardous chemicals

- reduce or eliminate civil and criminal liabilities under environmental laws

The program reflects the goals and policies for waste minimization for LBL and represents an ongoing effort to make waste minimization/pollution prevention part of the site's operating philosophy. LBL's overall efforts include assessment of waste minimization opportunities and the development of source reduction plans. The projected budget authority goals of the waste minimization program as included in the Environmental Restoration and Waste Management Program are indicated in the following table for LBL.'s unconstrained funding case. 
Waste Minimization Management Program (FY BA \$M)“

\begin{tabular}{lccccccccc}
\hline Category & 1993 & 1994 & 1995 & 1996 & 1997 & 1998 & 1999 & Total \\
\hline Operating & 0.2 & 0.2 & 0.6 & 0.6 & 0.7 & 0.7 & 0.8 & 3.8 \\
\hline
\end{tabular}

"Estimated ERWM (EX) Budget Authority for unconstrained funding case.

\section{National Environmental Policy Act Planning}

The Laboratory has undertaken a program to achieve full compliance with the National Environmental Policy Act and the California Environmental Quality Act. The Laboratory has prepared Guidelines for Compliance with the National Environmental Policy Act and the California Environmental Quality Act that describe processes and procedures for LBI scientists and managers to ensure compliance and to prepare documents and recommendations to DOE and to the University of California, Office of the President (UCOP).

I.BL's general plan of action for projects includes preparation of checklists specifying NEPA and CEQA documentation recommendations for Field Task Proposals and capital projects one to two years prior to funding, and for Work for Others, Cooperative Research and Development Agreements, and research grant proposals at the time of proposal preparation. The checklists are prepared by Division NEPACEQA Coordinators, in consultation with Principal Investigators, and are submitted to DOE in budget documents and to the LBL Office of Planning and Analysis for preparation and submittal of formal NEPA and CEQA documentation to DOE and UCOP.

During 1993 three NEPA Environmental Assessments were under development: the proposed Biomedical Isotope Facility, the Human Genome Laboratory, and Recycling Surplus Copper. The Laboratory also completed a Draft Supplemental Environmental Impact Report for the renewal of the operating contract between the DOE and UC.

\section{Safety and Support Services Facility}

Of vital importance to LBL's ES\&H program is the Safety and Support Services Facility. This project would provide mitigation of ES\&H services deficiencies by correcting inadequate physical resources for selected material handling and safety services needs. Some of the LBL safety functions, including instrument calibration, would be located in this structure. The building supports materials services functions, which are essential for safe and effective property control, and electronics instrumentation maintenance and quality control. An important element of the Safety and Support Services building is vacating an obsolete World War II era wooden building that is not rated for fire control.

Safety and Support Services Facility Resource Requirements (\$M)

\begin{tabular}{lccccccc}
\hline Category & 1994 & 1995 & 1996 & 1997 & 1998 & 1999 & Total \\
\hline Operating & 0.0 & 0.0 & 0.0 & 0.0 & 0.0 & 0.0 & 0.0 \\
Construction & 0.0 & 4.1 & 7.0 & 1.2 & 0.0 & 0.0 & 12.3 \\
\hline
\end{tabular}

"Estimate of actual your LBL. Budget Authority. 


\section{Environmental Monitoring and Industrial Hygiene Building}

To provide adequate space for environmental moritoring laboratories and offices, industrial hygiene offices and laboratories, and ES\&H training facilities, LBL requires a new ES\&H support facility, the [nvironmental Monitoring and Industrial Hygiene Building. This building responds to numerous and wide-ranging deficiencies in resources for ES\&H functions at the Laboratory identified by the Technical Safety Appraisal and Tiger Team and is included as part of the Corrective Action Plan described above. Many of these deficiencies relate to the lack of space, insufficient centralized resources, and inadequate staff. As indicated in the LBL. Tiger Team Assessment Corrective Action Plan, LBL must double its ES\&H staff to meet minimum standards of performance. LBL was found to have inadequate training facilities, ES\&H sampling and monitoring laboratories, and office space. The Environmental Monitoring and Industrial Hygiene Building is the centerpiece of LBL's program to provide the necessary resources for an effective ES\&H organization that meets compliance needs.

Environmental Monitoring and Industrial Hygiene Building Resource Requirements (\$M)

\begin{tabular}{lrrrrrrr}
\hline Category & 1994 & 1995 & 1996 & 1997 & 1998 & 1999 & Total \\
\hline Operating & 0.0 & 0.0 & 0.0 & 0.0 & 0.0 & 0.0 & 0.0 \\
Construction & 0.0 & 2.5 & 17.0 & 4.5 & 0.0 & 0.0 & 24.0 \\
\hline
\end{tabular}

"Estimate of actual-year LBL Budget Authority.

\section{ENVIRONMENTAL RESTORATION AND WASTE MANAGEMENT}

LBL environmental management site projects supported through the DOE Office of Environmental Restoration and Waste Management (EM) are essential to correct and restore environmental conditions at the Laboratory and to improve the management of waste handling operations in support of DOE's national environmental objectives. The corrective actions achieve and maintain required low exposure and risk levels; the environmental restoration program includes the assessment and characterization of contamination and the closure of the existing Hazardous Waste Handling Facility. Increased support for the waste management program is necessary for the proper management of radioactive and hazardous waste. The waste management program supports the construction of a new Hazardous Waste Handling Facility. These programs provide for compliance with DOE and other federal regulations and for meeting requirements established by state and local agencies.

The Laboratory's systematic and prioritized input to the EM Five-Year Plan supports DOE's national environmental restoration and waste management goals. The plan responds to specific environmental conditions at the Laboratory and includes facilitics and operating programs for managing 
those conditions to maintain air quality, surface water quality, and groundwater quality. The plan is focused on three Environmental Management programs for restoration and management activities:

- Environmental Restoration. Assessment, characterization, and remediation of chemical contamination of soils and groundwater and the closure of the existing LBL Hazardous Waste Handling Facility and the decommissioning of the Bevalac facility.

- Corrective Activities. Corrective actions to achieve compliance with environmental regulations that protect soils, groundwater, and air and also prevent chemical discharges to sewers. Essential corrections are to laboratory ventilation systems, deionization systems, sanitary sewer systems, chemical storage tanks, and wastewater treatment units.

- Waste Management. Waste Management's program for hazardous and radioactive waste handling, disposal, waste minimization, planning, and the construction of a new Hazardous Waste Handling Facility. Current funding projections as shown in the following table are adequate for meeting regulatory requirements. However, increased funding of waste management operations will be necessary to meet additional program requirements.

EM's Five-Year Plan is vital for compliance with DOE and other Federal regulations and for meeting requirements established by state and local agencies. The program has been developed in conjunction with DOE, state, and Federal reviews. The resource projections below and in Section 10 reflect existing guidance in Activity Data Sheets. These resources do not include additional corrective action planning requirements, maintenance projects, and other upgrades funded through LBL institutional resources. The resources also do not include the cost for decommissioning the Bevalac, following acceptance by EM, as described in Section 9. 


\begin{tabular}{lrrrrrrrrr}
\hline Category & 1992 & 1993 & 1994 & 1995 & 1996 & 1997 & 1998 & 1999 \\
\hline tnvironmental Restoration & & & & & & & & \\
Operating & 1.5 & 2.7 & 4.1 & 4.4 & 5.1 & 7.7 & 8.3 & 8.0 \\
Capital Equipment & 0.0 & 0.0 & 0.0 & 0.0 & 0.0 & 0.0 & 0.0 & 0.0 \\
GPP & 0.0 & 0.0 & 0.0 & 0.0 & 0.0 & 0.0 & 0.0 & 0.0 \\
Line Items & 0.0 & 0.0 & 0.0 & 0.0 & 0.0 & 0.0 & 0.0 & 0.0 \\
Total & 1.5 & 2.7 & 4.1 & 4.4 & 5.1 & 7.7 & 8.3 & 8.0 \\
EM Corrective Activity & & & & & & & & \\
Operating & 0.3 & 0.0 & 0.0 & 0.0 & 0.0 & 0.0 & 0.0 & 0.0 \\
Capital Equipment & 0.0 & 0.0 & 0.0 & 0.0 & 0.0 & 0.0 & 0.0 & 0.0 \\
GPP & 0.6 & 0.0 & 0.0 & 0.0 & 0.0 & 0.0 & 0.0 & 0.0 \\
Line Items & 2.8 & 0.0 & 0.0 & 0.0 & 0.0 & 0.0 & 0.0 & 0.0 \\
Total & 3.7 & 0.0 & 0.0 & 0.0 & 0.0 & 0.0 & 0.0 & 0.0 \\
Waste Management & & & & & & & & \\
Operating & 7.9 & 6.1 & 7.8 & 9.7 & 10.6 & 11.4 & 12.5 & 13.9 \\
Capital Equipment & 0.5 & 0.1 & 0.9 & 0.9 & 0.5 & 0.5 & 0.7 & 1.4 \\
GPP & 0.0 & 0.5 & 0.0 & 0.2 & 0.0 & 0.0 & 0.0 & 0.0 \\
Line Items & 1.6 & 0.0 & 5.8 & 0.6 & 0.0 & 0.0 & 0.0 & 0.0 \\
Total & 10.0 & 6.7 & 14.5 & 11.4 & 11.1 & 11.9 & 13.2 & 15.3 \\
Total EM Funding & 15.2 & 9.4 & 18.6 & 15.8 & 16.2 & 19.6 & 21.5 & 23.3 \\
\hline
\end{tabular}

aActual-year LBL Budget Authoiny as provided in the EM Five-Year Plan. The funding level shown for FY 1993 reflects the actual approved budget, for FY 1994 reflects the President's budget request to Congress, and for FY 1995 reflects the target (EM requested) budget level; proposals for decommissioning of Bevalac accelerators not included (see Section 9 for more details).

\section{LANDLORD FUNDED ES\&H}

ES\&H is currently funded through Laboratory overhead except in Waste Management and Site Restoration. Proposals for replacing overhead have been submitted through the ES\&H Five-Year Plan. To date, this process has not resulted in additional funding. It is, nonetheless, used as a basis for prioritizing allocations of overhead funding.

The Activity Data Sheet process makes the distinction between current, core activities and additional funding needed to fully implement an ES\&H program to meet compliance requirements and address real risks facing the Laboratory. A review of this year's Activity Data Sheet submissions makes clear the consequences of not receiving funds for compliance under the ES\&H Five-Year Plan. The Laboratory would be forced into noncompliance with Federal, state, and local regulations. This could lead to significant fines by the Federal Government and the State of California.

Additional funds have been subsidized by the Laboratory to cover Tiger Team activities. A very small amount of funds was received for Tiger Team activities in FY 1993. 


\section{TECHNOLOGY TRANSFER AND
SCIENCE EDUCATION}

\begin{abstract}
Create value for the economy, enhance education, and contribute to the community through partnerships with industry, universities, and other laboratories.
\end{abstract}

LBL Vision 2000

\section{TECHNOLOGY TRANSFER}

To support DOE's mission to increase the nation's technological competitiveness, LBL has strengthened its programs promoting technology transfer and science and engineering education. LBL has put in place numerous projects to help link its research and resources to private industry and to enhance its contributions to the nation's education system for the benefit of both teachers and students.

LBL streamlines its technology transfer organization. In FY $1992 \mathrm{LBL}$ restructured its technology transfer organization to increase its effectiveness. A new Technology Transfer Department (TTD) was established, reporting to the Associate Laboratory Director for Administration. The former Office of Sponsored Research Administration and the Technology Transfer Office now report to the head of TTD. The Associate Laboratory Director for Planning and Development continues to have responsibility for technology transfer policy, planning, and program development

The mission of the TTD is to provide ieadership, management and support for the transfer of $L B L$ technologies to the private sector.

A new TTD strives to make technology transfer an integral part of all LBL. programs. It identifies promising research early in the creative process and guides it through to its industrial application. TTD has comprehensive responsibility for providing technology transfer services to the divisions including work for others and user facilities agreements. The TTD works closely with the research divisions to select and promote technologies 
available for licensing or collaboration with industry. It negotiates intellectual property agreements and Cooperative Research and Development Agreements (CRADAs); manages the interface with licensees and CRADA participants; and oversees submission, approval, and tracking of sponsored research projects.

\section{Aggressive Technology Transfer Plan}

With technology transfer widely accepted as a mission of $L B L$, the Laboratory continues to expand, create and manage an organization and environment where technology transfer means business. LBL will effectively and aggressively translate its research and development projects into successful commercial products and services for the community and the nation. To accomplish effective technology deployment, LBL focuses on three critical elements: (1) innovative research and technical assistance, (2) outreach to strengthen industrial relations, (3) commercialization of intellectual property.

\section{Innovative Research and Technical Assistance}

DOE National User Facilities. To support the national infrastructure for fundamental science and engineering research, LBL provides to investigators from industry, universities, and government a range of unique research facilities and centers. In FY 1992 LBL had over 200 facility users and signed 43 user agreements for a total of $\$ 3.3 \mathrm{M}$. The major national facilities available to qualified investigators include:

- The Advanced Light Source, which will provide photon beams of unprecedented brightness and coherence and with picosecond time structure. The injector was commissioned in FY 1992, and the storage ring was commissioned in FY 1993. The facility will begin operation in fall of 1993.

- The 88-Inch Cyclotron, winich provides light ions, polarized protons and deuterons, and intense and high-charge-state beams of heavy ions (up to krypton) at energies up to about $35 \mathrm{MeV}$ per nucleon. The cyclotron facility has experimental areas for conducting nuclear science experiments as well as research in other areas such as biomedicine, atomic physics, and radiation damage in semiconductors.

- The National Center for Electron Microscopy, which consists of the High Voltage Electron Microscope, operating at up to $1.5 \mathrm{MeV}$ (highest energy in the U.S.); the Atomic Resolution Microscope, offering 1.5- $\AA$ resolution; and analytical microscopes and support facilities. An upgrade of the facility is planned (see Section 4).

- The National Tritium Labeling Facility, which provides advanced instrumentation to investigators needing high specific activities of tritiated compounds as tracers in chemical and biomedical research.

In addition to these national facilities, other research facilities involved in collaborative research include the Center for Computational Seismology, 
the Sky Simulator, the Mobile Window Thermal Test Facility, and the Low Background Counting Facility.

Collaborative Centers. The Laboratory has established programmatic research centers with specific objectives of fostering collaborative research with industrial and educational institutions. These include, for example, the Center for Advanced Materials, the Human Genome Center, the Center for X-Ray Optics, the Center for Computational Seismology, and the Center for Building Sciences.

The Center for Advanced Materials supports the goals of increasing U.S.-based industry in DOE research and has established research collaborations with related industries in surface science and catalysis (petrochemical), electronic materials (electronics), polymers (chemical), instrumentation for surface science (petrochemical and chemical), structural materials with an emphasis on light alloys (aerospace), and ceramic and metal interfaces (electronics). LBL's initiatives, such as the Atomic Scale Synthesis of Advanced Materials and Advanced Lithography initiatives, will provide a wide range of research opportunities and further extend these collaborations.

The California Institute for Energy Efficiency, a research unit coordinated by the University of California, has been formed to provide a vehicle for improved technology transfer and cooperative research support. The Center is developing efficient end-use technologies to benefit users, utility companies, and manufacturers.

Work for Others. LBL is engaged in $\$ 40 \mathrm{M}$ worth of sponsored research agreements that are non-DOE funded. Examples include:

- $\$ 16 \mathrm{M}$ per year from the NIH to support various research projects in the Life Science Division.

- $\$ 10 \mathrm{M}$ per year (\$57 M total over 5 years) from ARPA to build a beamline to test optical surfaces and coatings and support modern materials fabrication at LBL's Advanced Light Source (ALS) User Facility.

- \$1.1 M from EPRI and GRI for research in energy and environment at LBL.

- $\$ 250 \mathrm{~K}$ from IBM for a partnership with Tulane University and Tennessee University for the construction of a partial beamline at the ALS.

Training Scientists of the Future. Finally, LBL believes that supplying technical and engineering training to the students who will ultimately apply their skills in industry, government, and universities is of vital importance to the Laboratory and the nation's future. Located adjacent to UC Berkeley, LBL has established a strong relationship with the University community. More than 650 advanced students are supported at LBL. About 100 students annually receive advanced degrees based on $L B L$ research, and approximately half of these students bring their technical talents to industry. LBL's numerous science and technology education programs are discussed later in this section. 
Technology Transfer Effort (Estimated)

\begin{tabular}{|c|c|c|c|c|c|c|c|c|}
\hline Category & $\begin{array}{c}F Y \\
1992\end{array}$ & $\begin{array}{c}\mathrm{FY} \\
1993^{\mathrm{a}}\end{array}$ & $\begin{array}{c}F Y \\
1994^{\mathrm{a}}\end{array}$ & $\begin{array}{l}F Y \\
1995\end{array}$ & $\begin{array}{c}\text { FY } \\
1996\end{array}$ & $\begin{array}{l}\text { FY } \\
1997\end{array}$ & $\begin{array}{l}\text { FY } \\
1998\end{array}$ & $\begin{array}{l}F Y \\
1999\end{array}$ \\
\hline \multicolumn{9}{|l|}{ Activity } \\
\hline $\begin{array}{l}\text { Industry Cooperative } \\
\text { Agreements }\end{array}$ & 13 & 15 & 20 & 25 & 25 & 25 & 25 & 25 \\
\hline $\begin{array}{l}\text { Agreement Value } \\
(\$ M)^{\mathrm{b}}\end{array}$ & 6.0 & 6.2 & 12 & 18 & 20 & 24 & 30 & 36 \\
\hline Personnel Exchanges & 2 & 3 & 5 & 7 & 7 & 7 & 7 & 7 \\
\hline \multicolumn{9}{|l|}{ Staffing } \\
\hline $\begin{array}{l}\text { Technology Transfer } \\
\text { Department }{ }^{\mathrm{C}}\end{array}$ & 4 & 6 & 12 & 14 & 14 & 14 & 14 & 14 \\
\hline
\end{tabular}

aEstimated approved (CRADAs only).

bThis represents total dollars awarded by calendar year (vs. funding received annually).

${ }^{\mathrm{C}}$ Includes Office of Sponsored Research Administration, promotion, and licensing efforts.

\section{Partnerships with Industry}

Industrial Relations/Outreach. The outreach objective at $\mathrm{LBL}$ is to increase the recognition of $L B L$ as a major resource for new technologies and partnerships. To promote LBL's technologies, the TTD uses a broad range of communication tools, from press releases and brochures to exhibiting at trade shows. LBL plans to increase the use of video tape and advertisements to announce CRADA opportunities. As a result of successful marketing efforts, LBL received over 1000 inquires from the private sector in FY 1992. Each inquiry receives personal, one-on-one contact by letter, phone or by

This infrared image demonstrates the dramatic temperature difference experienced by a person entering a vehicle parked in direct sun. $L B L$ researchers are investigating automotive applications for advanced insulation, window glazing, and electrochromic devices to reduce direct solar radiation and lower interior temperatures.

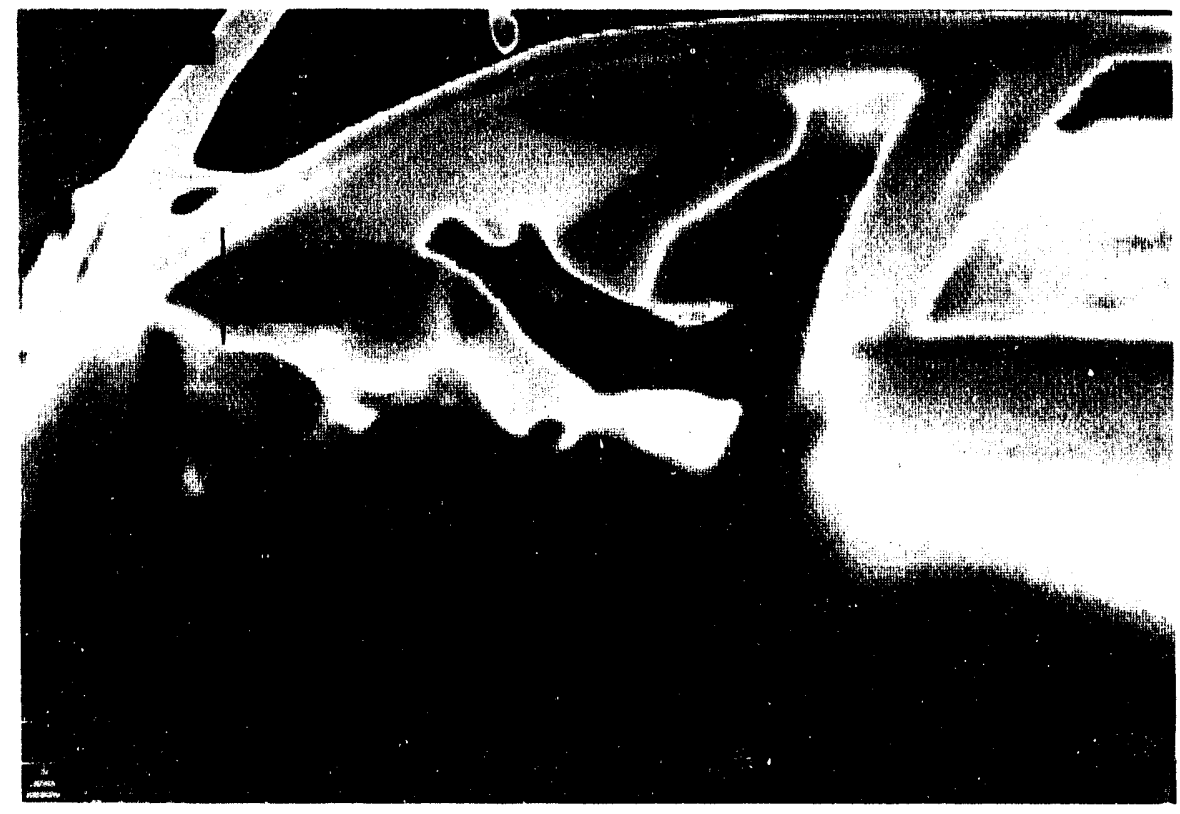


visiting $L B L$ facilities. An LBL inquiry database is maintained to track interactions. One example of a successful communications effort is the "New Technology Announcement." The attributes of a new invention available for licensing or collaborative partnership are summarized using attractive layouts and highlighting potential product applications. The package is sent to the corporate executive in charge of company development, targeted through a high-technology corporate database. LBL also distributes the announcements to emerging technology newsletters and releases them to trade journals and the general press.

Other efforts to strengthen ties with industry include:

- A collaboration with the Haas School of Business on the UC Berkeley Campus to initiate several new projects in the MBA Engineering and Technology program. Students work with the UC Business School and TTD to develop economic market data that will help attract industry interest to LBL technologies.

- An advisory board membership with East Bay NET (a professional network for entrepreneurs and technologies) to promote early-stage technologies for first-round, start-up ventures. The group holds semiannual briefings to showcase new technologies to local venture capitalists. As a member of East Bay NET, LBL presents its latest promising technologies with strong potential for licensing or the formation of start-up companies. East Bay NET holds bimonthly meetings with technology transfer, venture capital, and business service professionals.

- LBL will continue to participate in the Technology Transfer Personnel Exchange Program, which promotes collaborative technology research projects with industry. This program provides financial assistance for extended visits by senior American industry scientists to the national laboratories. These visits have been highly productive, and the Laboratory has requested program expansion. Several of the staff exchanges have resulted in executed CRADAs with the industrial partner.

Because outreach involves Laboratory networking, internal programs will focus on the important opportunities to perform collaborative research with U.S. industry. One way in which this is being accomplished is by having in place a technology transfer liaison, a senior division manager or technology transfer specialist, who acts as a single point of contact within each of the 12 research divisions to help disseminate technology transfer opportunities, updates, technology inquiries, and requests.

"Spin-Off" Technologies. LBL proposes to expand the scope of its efforts to accelerate and strengthen the transfer of small spin-off research and technology to private industry through the DOE's Energy Research Laboratory Technology Transfer program (ER-LTT). LBL's ER-LTT projects are designed to transfer technology spin-offs directly from LBL research program activities or support further technology research or maturation necessary to develop a technology to the point where a private sector partner can evaluate it for commercial applications. A spin-off technology is defined as an "orphan" technology that does not fit the funding plans of another programmatic area. For FY 1993, LBL has submitted 51 spin-off CRADA proposals of which industry is willing to commit approximately $\$ 40 \mathrm{M}$ to the ER-LTT program. 
Comparing leg tendon tissue isolated from 16-day-old chicken embryos (bottom left) and tissue from a 4month old chicken illustrates one of the two critical changes that occur as the organism develops-a drop in cell number. Correspondingly, during this period of rapid growth, the capacity to produce collagen is dramatically reduced. The elucidation of the developmental switch responsible for promoting the production of collagen-a major building block of tendons and other connective tissues-is the focus of a CRADA with Amgen Inc., a global biotechnology leader.
Consortia Collaborations. A new ER-LTT program component will build upon these program options with the addition of larger collaborative projects to the LBL's technology transfer portfolio, such as consortia involving several laboratories and multiple industry partners focused on a broad research agenda addressing diverse technology research areas. One example is LBL's highly successful technology partnership with California's universities and major utility companies. Known as the California Institute for Energy Efficiency, the consortium was established to respond to California's energy and environmental needs by tapping the scientific and technical capabilities of the state's universities, colleges, and universityaffiliated laboratories in developing new energy-efficient technologies for buildings, industry, and transportation.

The California Comeback. LBL. will share the challenge to revitalize California's economy. LBL intends to pursue action agendas that will create advanced technology partnerships with California universities, Lawrence Livermore National Laboratory and the State's private industry. As a great state resource, California can tap LBL's expertise in biotechnology and life sciences, advanced materials, environment, energy, computing and communications, and transportation. In the spirit of the National Technology Initiative, LBL will host or co-host workshops/conferences to help identify emerging technologies and match-up beneficial partnerships that will in turn translate to sustainable increases in high value-added jobs and a competitive California.

California start-up companies based on LBL technologies:

- PolyPlus-Berkeley, CA company formed to develop rechargeable, solid-state lithium batteries

- Conductus, Inc.-Sunnyvale, CA company formed to develop practical applications of LBL's high $T_{c}$ superconductor technology

- ISM-San Diego, CA company started to manufacture vapor deposition system

- Thermalux-Emeryville, CA company started to develop Aerogels

- Morris Research-Berkeley, CA company formed to perform superconductor research

- Southwall-Palo Alto, CA company formed to develop window glazing technology and custom thin film technologies
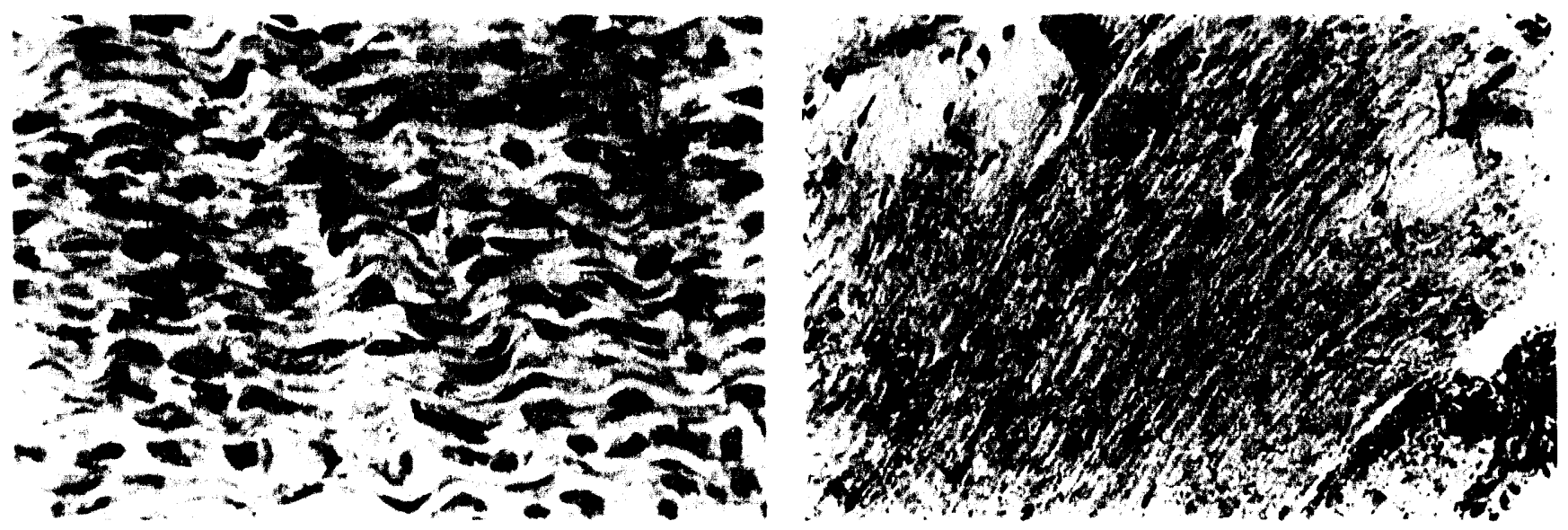
CRADAs with companies in the state of California:

- Conductus-Sunnyvale, CA company working with LBL to develop SQUIDs

- Amgen-Thousand Oaks, CA company collaborating with LBL. to develop a drug that will speed the repair of tendon and ligament damage

- Seagate Magnetics-Fremont, CA company that manufactures computer disk drives is working with LBL to develop superior computer hard drives

- California Institute for Energy Efficiency (CIEE)-a group of California utility companies formed to collaborate with LBL and UC to develop energy efficient technologies such as better insulation products

- Gilycomed-Alameda, CA company working with LBL to develop new carbohydrate-based materials

Industrial Development Program. The Industrial Development Program effort is an outreach of the Technology Transfer Department that is staffed with personnel that have scientific and industrial backgrounds. Internally, LBL's evaluation of new scientific and technological results is coupled to markets and specific industrial candidates. Expertise and strategies for presentation of LBL capability, including technology transfer mechanisms, is then presented to industry and/or local institutions jointly with the scientific team. The manager of Industrial Program Development usually works with industry Vice Presidents and Presidents to structure the interactions. Externally, Industrial Program Development watches the marketplace and identifies market driven opportunities where the LBL infrastructure and expertise can assist economic development. Since the office is outside the scientific program, interdisciplinary themes can be developed because of the unimpeded access into all of the programs.

To maximize the effectiveness of the LBL/ industry efforts, the resources of $L B L$ should be focused where the greatest leverage is gained from a match between Laboratory talent and infrastructure, and industry needs. The Laboratory would benefit most from a focus on local industries.

For example, biotechnology is a well-matched industry. The biotechnology industry was born out of the University of California San Francisco, University of California Berkeley, Stanford, and Lawrence Berkeley Laboratory. The greatest concentration of biotechnology companies in the U.S. is located in the Bay Area and in California. This high growth industry is expected to create $1 / 3$ of the new jobs in California $(200,000)$ over the next five years. The industry is already closely tied to existing scientific programs with LBL, including the Human Genome Center, the Materials Sciences Division, the Structural Biology Division, the Life Sciences Division and the Advanced Light Source. These divisions and centers employ a substantial fraction of the scientific and technical staff of the laboratory. This synergy of human resources, a growth industry, unique physical infrastructure, and an industry with positive growth potential can keep the U.S. at the top of the international biotechnology industry. L.BL intends to contribute to the success of this exciting field.

\section{Commercialization}

Intellectual Property. The third element of LBL's technology transier strategy is the commercialization of intellectual property. In addition to increasing its outreach efforts and encouraging its scientists to become involved in tech transfer opportunities, LBL. has developed a "Technology and Invention 
Inventory," which lists LBL technologies (including patentable inventions, public domain technologies, and copyrightable works) in database form. The database yields information regarding subject area, patent and licensing status, and scientists. Portfolio categories of technologies that interest U.S. companies include battery teclinology, chemical processes, new materials, advanced particle and radiation detectors, energy efficiency technology, ion sources, new instrumentation, medical applications, software, biotechnology, and environmental technology. The Laboratory is taking steps to identify potential intellectual property early in the creation process, to encourage its development, and to ensure its protection through appropriate means. The technology transfer staff is working closely with the LBL Patent Department to ensure the early identification of commercializable ideas.

Patents and licensing. LBL seeks to patent and license its intellectual property to strengthen the value of its inventions, both for use and applica. tion by industry and to promote the research and technology transfer interests of the Laboratory and its research staff. L.BL made 20 patent applications in FY 1992, and 15 patents were issued.

LBL licensing activity has increased significantly since the 1980 s when relatively few agreements were made during the decade. Over the past two fiscal years, seven license agreements (including options) have been made and nine more are estimated for 1993.

Licensing income typically arises from three terms in the license agreement: an upiront onetime, license issue fee; minimum annual royalties; and sales royalties. Intellectual property from a research laboratory may require considerable development effort. Thus, sales royalties are not expected to begin until several years after the license is signed. Licensing and royalty income: distributed according to University of California policy and is consistent with patent law. Patent prosecution costs are first deducted and University policy allows the Laboratory to assign 15\% for administrative costs in maintaining the license and licensing effort. After the inventor receives a share, the remainder is available for Laboratory Research. Consistent with patent iaw, 75\% of the royalty income that exceeds $5 \%$ of LBL's annual budget will be paid to the U.S. Treasury. LBL evaluates the allocation of intellectual property rights in technology transfer agreements.

Intellectual Property Management

\begin{tabular}{lcrrrr}
\hline Category & FY 1992 & $\begin{array}{c}\text { FY 1993 } \\
\text { (est.) }\end{array}$ & FY 1994 & $\begin{array}{c}\text { FY 1995 } \\
\text { (est.) }\end{array}$ & FY 1996 \\
\hline New Licenses (No.) & 5 & 16 & 20 & 21 & 22 \\
License Income (\$K) & 79.8 & 344 & 240 & 280 & 350 \\
Patent Applications & 20 & 25 & 25 & 30 & 33 \\
Patents Issued & 15 & 20 & 20 & 21 & 23 \\
Patent Staffing & 1 & 2.5 & 3 & 3 & 3 \\
Intellectual Property & Use of income & & & & \\
Management & & & & & \\
\hline
\end{tabular}

\footnotetext{
"Includes options.

"Invention \& ORTA Administration: 15\% of gross income plus paltenting costs

Scientific or Applied R\&D: $50 \%$ of net income

Awards \& Inventor Payments: $50 \%$ of net income Fducation/Training: ()

()ther (Specify): 0
} 


\title{
Technology Transfer Achievements
}

\author{
Industry-Laboratory Cooperative Projects
}

LBL is engaged in \$40 M worth of industrially sponsored research agreements that are non-D) (unded. The Laboratory has also initiated a number of lechnology research participation programs to improve the transfer of emerging energy lechnology to private industry. This includes forefront developments in advanced nanostructures fabrication and novel biomedical instrumentation. The Laboratory is also supporting new CRADA opportunities and initiating pilot projects to promote cooperative research opportunities within specific program areas. These projects have been successful in altracting U.S. corporate interest. Currently L.BL has 21 signed CRADAs totaling \$32 M with private industry. Examples are included below.

CRADAs in progress include: Conductus, Chiron, DuPont, Ford/Rouge Steel, Dow Chemical, DEEP, and Glycomed.

LBL joins American Textile Collaboration. With the signing of a multimillion dollar cooperative research agreement, LBL joined the DOE and seven other national laboratories to help the U.S. textile indusiry develop high technology that can successfully compete with foreign labor--thereby saving thousands of jobs and creating thousands more. The historic effort is a result of the Critical Technologies Workshop that was organized in Berkeley last spring by L.BL. Representatives of the textile industry and DOE formed a partnership called the AMTEX. It is through this partnership that the newly signed CRADA was forged. The CRADA calls for $\$ 30$ million to be spent in the next six months-and could reach $\$ 200$ million within five years (with DOE and industry contributing equal ainounts of money)-on research aimed at reversing a trend that has cost this nation more than halfa-million jobs during the past ten years. Two million dollars of the CRADA's FY 1993 money is earmarked for LBL. The AMTEX CRADA lists five major areas of research to be investigated: improved materials and processes, de mand-activated manufacturing; environmental quality and waste minimization; energy efficiency; and automation.

\section{Representative Accomplishments}

\section{Preparation and Characterization of Novel Lubricated Hard Carbon} Coatings for Computer Hard Disks. LBL has signed a CRADA with IBM in which hard carbon thin films will be deposited on various single crystal substrates (silicon, silic on dioxide, platinum) using plasma assisted chemical vapor deposition and sputter deposition. The structure, mechanical proper. ties (adhesion, friction) and chemical properties (adsorption or organic monolayers, resistance to oxidation) will be determined on the atomic level using modern surface science lechniques (STM, AFM, XPS, LEED, HREELS, ALS, SF (i, ISS) that are, in combination, unique to our Laboratory at L.BL's Center for Advanced Materials. These studies will lead to the development of the next generation of lubric ated hard coating technoleggy for disk drives for the U.S. computer industry.

Raman Spectroscopy Characterization of Amorphous Carbon Coatings for Computer Hard Disks. I.BL. and Seagate Magnetics, a Fremoni, California firm that manufactures computer disk drives, are jointly developing a new prosess for making c arbon coatings that will protect the next generation of 
hard disks. The goal is to develop new types of amorphous carbon coatings that can be applied during the production of a hard disk's thin magnetic layer. Considered a key requirement for increasing the storage capacity and reliability of hard drives, these coatings protect a magnetic layer from wear and tenr that occurs during the startup and operation of a computer.

Critical to achieving the goal of the LBL-Seagate CRADA will be the ability to characterize the carbon coalings produced at the atomic level. Such characterizations reveal much about the relationship between atomic structure and properties in a material and can lead to ways of optimizing manufacturing processes to improve the material's performance.

Scientists at LBL have developed a unique Raman spectroscopy lechnique whereby properties associated with changes in the position of aloms with respect to one another across the surface of a material can be determined thousands of times faster than ever before. This technique along with other research findings will be passed on to experts at Seagate who will apply it to their work. LBL and Seagate scientists and engineers will also collaborate on the design of new experiments that will further increase the value of Raman spectroscopy in the field of computer disk drive manufacturing.

\section{SQUID Applications of High Temperafure Superconducting Thin Films.} LBL and Conductus, Inc. of Sunnyvale, California have signed a CRADA, the goal of which is to build and test a prototype system involving one or more high transition temperature $\left(T_{c}\right)$ Superconducting QUantum Interference Devices (SQUIDs), each coupled to a high- $T_{C}$ flux transformer, to form a sensitive magnetometer. Potential applications of the system include geophysics surveying, nondestructive evaluation and biomagnetic measurements. The SQUID and flux transiormer may each be on a separate chip to form an integrated magnetometer. Both the SQUID and the flux transformer involve epitaxial multilayers of the high- $\mathrm{T}$, superconductor $\mathrm{YBa}_{2} \mathrm{Cu}_{3} \mathrm{O}_{7-x}$ and of buffer and insulating layers such as $\mathrm{CeO}_{2}$ and $\mathrm{SrTiO}_{3}$. These films are grown in situ on substrates at a temperature of about $800^{\circ} \mathrm{C}$ using a pulse excimer laser. It is particularly important to obtain high-quality, low. • noise films and junctions to ensure a high-magnetic field sensitivity.

\section{Metric Conversion Program}

Recognizing the value of adopting the common worldwide language of measurement, Lawrence Berkeley Laboratory has embarked on a program to implement the use of the metric system throughout its operations. It is the Laboratory's policy to require the use of the modern metric system, the International System of Units (SI), exrept when safety considerations dictate otherwise or when metric usage would entail excessive costs or otherwise seriously impede Lab operations. Exceptions are allowed for programs whose DOE sponsors specify the use of the inch-pound system. Division directors have broad responsibility for the course and pace of metrication within their respective divisions. The goal for $\mathrm{LBL}$ as a whole is to convent completely to metric usage within the next few years.

A number of the organizations have already begun the transition to metric usage. These groups include:

- Stores/Purchasing, which is developing a system for procuring and storking metric items in addition 10 inch-pound items.

- Mechanical Engineering, which is taking the lead in specifying a metric fastener standard for the Lab. 
- The Electronics Maintenance Ciroup, which calibrates electronics meters and other equipment based on the NIST's SI 90 standard for the volt.

- The Mechanical Processes Department, whose computer-controlled machines operate in both metric and inch-pound modes.

- The Maintenance and Operations Group, which is already providing many of its services using metric materials and measuring tools.

- The Facilities Department, which will use metric units in forthcoming Conceptual Design Reports and all other new design documents.

- The Environment, Health and Safety Division, which will include metric units in its upcoming revision of PUB-3000.

Metric units are to be used in all LBL reports and public ations. Exilusive use of $\mathrm{SI}$ units is preferred wherever possible, but dual dimensioning is allowed during the transitional period. Where dual dimensions are used, the preferred format is SI units first and the corresponding inch-pound units in parentheses. For cases in which safety is a prime concern, publications may provide dual dimensions with inch-pound units first and SI units following in parentheses. The ASTM document E380.92, Standard Practice for Use of the International System of Units (S)) the Modernized Metric System), will be the Lab's guide for metric practice.

\section{SCIENCE, MATHEMATICS, AND ENGINEERING EDUCATION}

\section{Overview}

As a national laboratory entrusted with advancing the nation's science and teclinology base, LBL is dedic ated to using its resources to help meet national needs in education. To this end, LBL's Center for Science and Engineering Education has aligned its mathematics, science and technology education mission and goals with the strategic goals of the Federal Coordi. nating Council on Science, Engineering and Technology. The Center for Science and Engineering Education was established to expand LBL's role in science education at all levels, and to serve the diverse population of students in U.S. schools. The Center's mission is to develop, implement, and evaluate programs which fully exploit the rich and unique scientific, lechnical and administrative resources of the Lawrence Berkeley Laboratory.

\section{Education Partnerships: A Strategy for the Future}

LBL has developed regional and national partnerships to improve the quality of mathematics, science, technology, and engineering education for the community and the nation. These partnerships represent diverse popu. lations and have brought about fundamental changes in the quality of education for tens of thousands of students and more than a thousand faculty and teachers over the last ten years. Our strategy has been to pursue partnerships that serve K-12 students, teachers, faculty, school ardministrators, school districts, and universities at both the undergraduate and graduate level. After six years there is clear evidence that these partnerships have had a positive impact on the quality of math and science education for these served by our programs. 


\section{Model Partnership with Minority Institutions}

In 1983 a Science Consortium was formed linking LBL. lackson Stute University (ISU), and the Ana (i. Méndez Universily System (ACiMUS). This Consortium is the first and oldest partnership among a Department of Energy National Laboratory, a Historically Black University, and a Hispanic Univer. sity System. In the 10 years since it was founded, the LBL/ISU/ACiMUS Science Consortium has focused on six major areas needing attention. These areas are listed below along with a summary of the accomplishments of the Consortium.

- Student Development. In 1983, few students from ISU and no students from ACiMUS participated in energy-related research and development activities on campus or at DOE national laboratories. By 1993, over 210 students participated in over 25 ) research appointments at LBL. alone, and many others have gone to other national laboratories or were student research assistants on campus.

- Precollege. In 1983, ACMUS and ISU had no precollege outreach programs in the schools of science and technology on their campuses. In 1993, hundreds of teachers and students annually benefit from summer precollege outreach programs as a result of the Science Consortium's support.

- Faculty Development. In 1983, ISU had no formal School of Science and Technology faculty-development program, and few ISU faculty had been to DOE national laboratorics. Of the 36 ACiMUS science faculty members, 1 had a doctoral degree, 30 had master's degrees, and 5 held bachelor's degrees. No faculty members at ACMUS and lew at ISU had peer-reviewed publications in the fields of energy, environment, mathemalics, and computer sciences. In 1993, the majority of the ACINUS faculty have doctoral degrees and are aclively involved in collaborative research. ISU faculty members are oblaining competitive research grants, annually publishing peer-reviewed papers, and allending conferences and workshops.

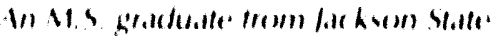

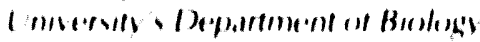

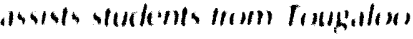

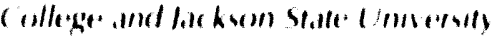

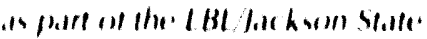

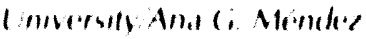

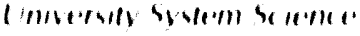

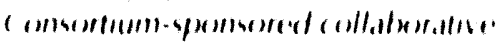

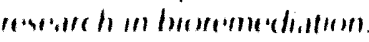

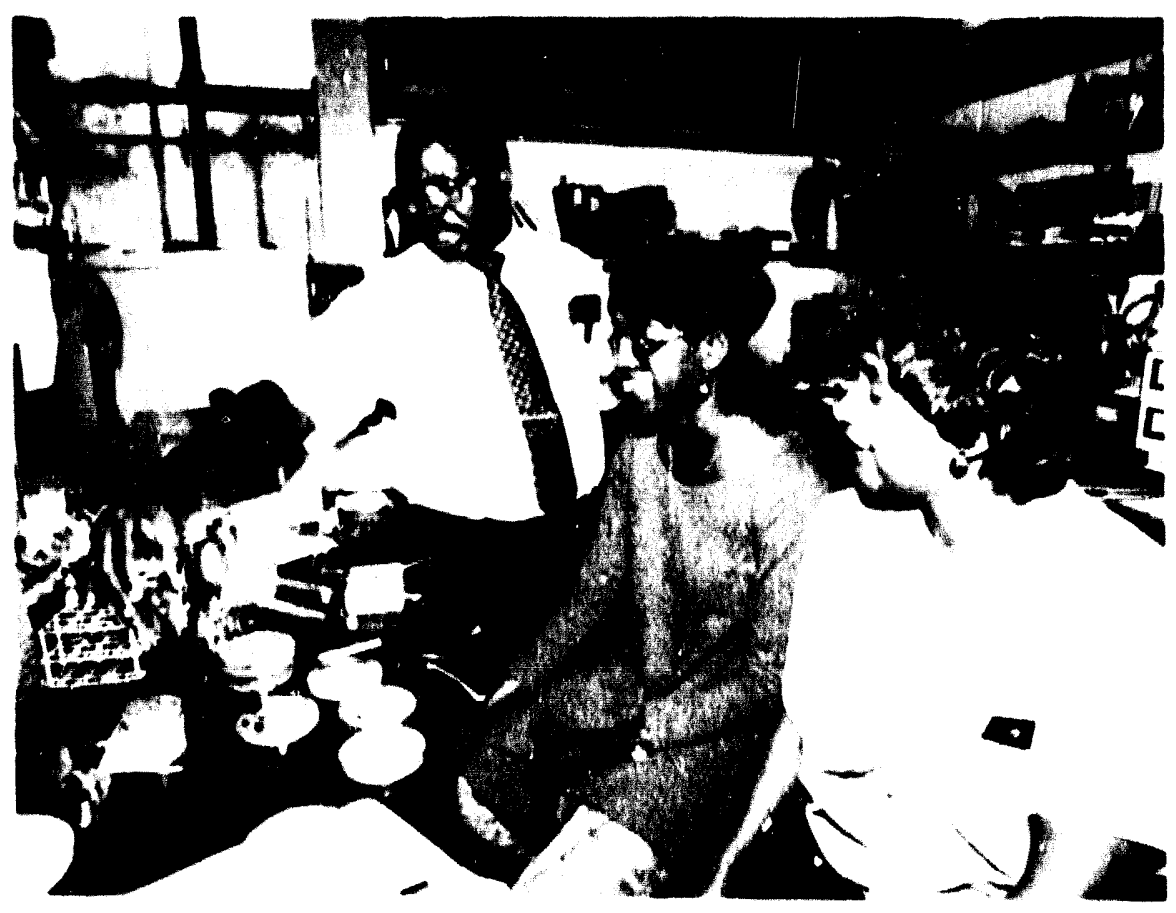


- Curriculum Development. In 1983, ACiMUS offered only an associate degree in the natural sciences, and ISU lacked computers and labora. tory equipment to suppont the bachelor of sciences degrees in the school of science and technology. The computer science curriculum was outdated in relation to the rapid advances in computing capabil. ity. By 1993, ISU and ACMUS had developed a modern curriculum in the sciences and computing sciences that includes the use of modern equipment.

- Research. In 1983, ACiMUS did not have a research capability in science or computer science. ISU had little energy research and development activity and no collaborative research. By 1993, col. laborative research activities involving $L B L$ scientists had led to nationally recognized publications, follow-on research grants from federal agencies, and opportunities for students to be involved in forefront research projects. The most successful of the projects established Bioremediation Education, Science and Technology (BEST) Centers at the three institutions and led to a Memorandum of Under. standing with the School of Natural Sciences of the University of California, Berkeley, to carry out advanced research and development in this emerging field.

- Infrastructure Development. In 1983, ISU and AGMUS had inadequate computing and networking capability to support scientific research or science and technology education. ACiMUS lacked adequate facilities for faculty and student research. By 1993, both institutions had local area networks, and many faculty members were using Internet. Scientific computing was strengthened by VAX computers, some of them donated by LBL, and science teaching and research facilities had been opened at all three ACMUS campuses.

LBL was able to further advance its collaborative research activities by expanding the partnership to include University of California at Berkeley's school of Natural Resources. This has enhanced student and faculty development, as well as curriculum, and has overall served to strengthen the science departments at the consortium universities. It has also led to the creation of a very successiul model for research and education, the BEST Center. Two BEST Centers, one at LBL and the University of California at Berkeley, and one at lackson State University will provide faculty and students from predominantly minority institutions across the country the opportunity for training in bioremediation techmology. A third center will be established in Puerto Rico at the Mendez Universities. These centers, which are largeted to be funded under the defense conversion plan, are a result of the Science Consortium's support for a collaborative research project involving over six faculty and staff and ten students from three institutions. Work was done primarily at LBL and UCB during intense summer research campaigns. The collaboration has led to important new information in the field of bioremediation.

BEST Centers will be foc al points for the development of new bioremediation education, research, and technology. The centers will devise and implement new curricula and educational programs for the highly specialized needs of the bioremediation field, incorporating biosience, chemistry, geologic al ac iences, and engineering! components, developing and disseminating new lechnologies for the remediation of halardous metal and solvent wastes. In conjunction with BEST Industrial 
An LBL nuclear physicist gives Oakland high-school students an introduction to the way an accelerator works. Through the $B A S T E C$ alliance, $L B L$ scientists and rechnicians are able to share their knowledge and expertise to advance $K-12$ science education in the ()akland Unified School District.
Partners, the new methodologies will be applied to remediate key pollutants and improve the environmental quality for the people of California, Mississippi, Puerto Rico, and the nation. Ultimately, the BEST Centers will provide both minority students and faculty with the opportunity to work on the forefront of science and technology and to make significant leadership contributions to the technology and manufacturing base of the country.

\section{The Bay Area Science and Technology Education Collaboration (BASTEC) - A Partnership with the Oakland Unified School District}

In the fall of 1989. DOE's education program identified partnerships with school districts as a high-priority opportunity for its national laboratories. LBL, LLNL, SLAC and Sandia/California began to meet to discuss the possibilities of working with the Oakland Unified School District. The Oakland District serves over 55,000 students representing over sixty ethnic groups and languages. The student ethnic composition is $57 \%$ African American, 16\% Asian, 15\% Hispanic, 9\% Caucasian, and 3\% other. BASTEC is starting its fourth year under a grant provided by the U.S. Department of Energy to Lawrence Berkeley Laboratory. Beginning with a yearlong series of round table meetings and workshops with teachers and administrators in late 1989 and early 1990, the Oakland Unified School District entered into the BASTEC collaboration with four San Francisco Bay Area Department of Energy National Laboratories, the Lawrence Hall of Science and 16 other Universities and Organizations. These partners were already active supporters of mathematics, science and technology education in the District, but they rarely interacted with each other. In the first year of funding, a conference was held with 120 teachers from the District to assess their needs. In the middle of that school year another "mini-conference" to update teachers' knowledge and instruction methods was held, with over $450 \mathrm{~K}-12$ teachers attending, far more than expected. Teacher leaders from

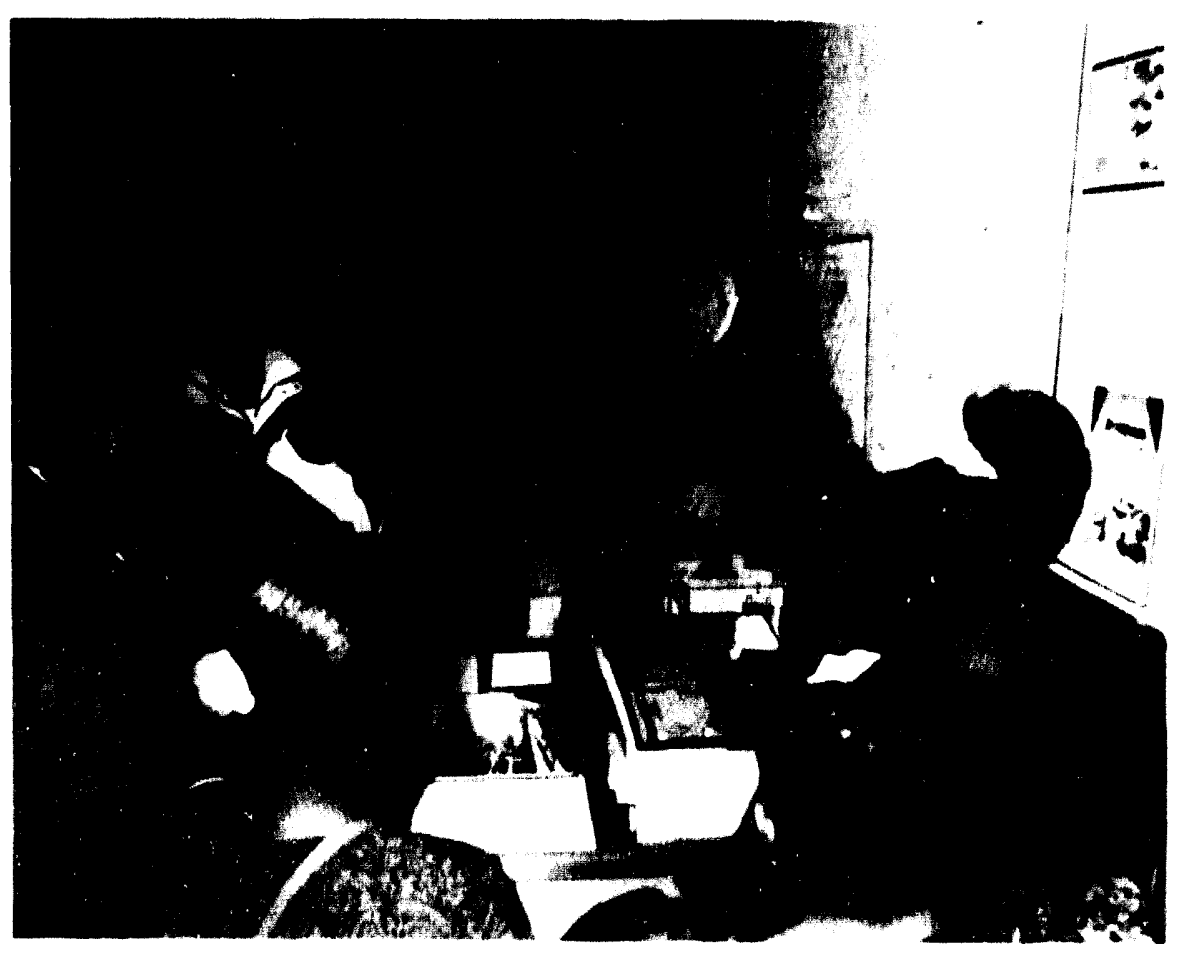


the district provided many of the workshops, and every participant received a copy of the new Science Framework for California Schools. Mini-grants to teachers provided during the school year and the establishment of a resource center demonstrated BASTEC's responsiveness to teachers' immediate needs for materials, supplies, equipment, and field trips. The partnership supported a Science and Technology Awareness Day, and Dr. Mae Jemisson, the first African-American female astronaut, was the featured guest at the event that reached well over 5000 students. Summer workshops for over 250 teachers rcunded out the year's events, with 12 workshops designed to improve teachers' knowledge, teaching skills, and curriculum, as well as their ability to tap into the science resources of the Bay Area.

At the start of BASTEC's second year with the Oakland Unified School District, the District's Superintendent invited BASTEC to develop the K-12 Curriculum for the District. This was a tremendous vote of confidence by the District for BASTEC. Recognizing that teacher acceptance is the key to implementation, BASTEC set up a series of four Saturday workshops and established grade-level and subject-area working groups, eventually involving over 100 teachers in the science curriculum writing process, as well as scientists, parents and science educators. The second mini-conference was limited to 450 teachers. Representatives from surrounding school districts attended the conference to solicit presenters for their own district conferences. The number of mini-grants was doubled in response to the large number of high quality proposals received. A second Science and Technology Awareness Day provided several thousand students with hands-on science lessons sponsored and provided by business and industries. Over 250 teachers again participated in multiple-week workshops provided by BASTEC's partner institutions.

During the third year of BASTEC, the mini-conference, mini-grants, and Science and Technology Awareness Day were continued. The science core curriculum was delivered to the Superintendent and School Board. Following a review, the BASTEC-developed Core Science Curriculum was promoted by the Superintendent as a model for other subject areas. Summer workshops for over 250 teachers were designed to support the core curriculum and the District applied its own Eisenhower math/science funds to expand the number of participants in the summer workshops. BASTEC will begin working with the District on a formal assessment of its systemic impact on teacher performance in 1994.

\section{Partnerships for Professional Development of Teachers of Science K-12 - The DOE National Teacher Research Associate Program}

DOE's nationwide Teacher Research Associate Program had its roots at LBL and became a national program in 1985 by including Argonne National Laboratory and Brookhaven National Laboratory. In 1993, over 26 Department of Energy laboratories provided over 226 high school and middle school science and mathematics teachers with an eight week summer research appointment. Of those 226 teachers, 44 came to LBL from both Bay Area schools across the country. While at LBL they spent four days with their assigned research teams and one day each week participating in laboratory research and curriculum development workshops. The program has served to give science and mathematics teachers the opportunity to be scientists, sometimes for the first time in their lives. They update their knowledge of science, learn how scientific groups operate on a day-to-day 
basis, and validate the important teaching methods relevant to preparing students to live and work in a technically advanced culture. At the same time, many scientists at the Laboratory are able to gain some insight into what is happening in the classroom and tune in to science teachers needs. This has resulted in greater involvement of the Laboratory's scientists in the education reform effort. One such "spin-off" has been the establishment of an NSF funded "Hands-On Universe" project that will give teachers and students access to the automated astronomy technology used by LBL astrophysicists to search for supernova. Over 210 teachers have participated as teacher research associates in the LBL program since 1983. Similar programs were started at other laboratories and by business partnerships and have gained credibility as a complement to traditional two and three week workshops for professional development.

A second partnership, the Science Education Academy of the Bay Area (SEABA), was established through the signing of a Memorandum of Understanding at Lawrence Berkeley Laboratory in January of 1992. The Academy is now funded primarily under a grant from the U.S. Department of Education, with over sixty-two institutions in the San Francisco Bay Area. The Academy is committed to providing in-service education and professional development to over 40,000 teachers over the next 10 years, helping K-12 science educators implement the new Science Framework for California Public Schools. A major objective of SEABA is to establish an electronic. catalog of staff development opportunities for the teachers. A data base of teacher professional development will be tracked and made available to teachers and school administrators for recognition and assignment of leadership responsibilities. SEABA will provide forums for members to share best practice and for educators to update members on innovations in science, mathematics and technology education. LBL played a central role in the establishment of SEABA and will benefit from this network to reach and serve the Bay Area teachers.

\section{Partnerships with the Community}

In 1992 LBL formed a new outreach project to provide LBL staff with direct access to students in the classroom though volunteer opportunities. The program partners with community organized volunteer projects. The number of volunteers contributing time and resources to these local volunteer organizations has grown from 33 to over 100 people, providing up to six hours weekly of volunteer service to assist students and teachers. Training programs for the volunteers are provided, and a newsletter keeps the staff informed of activities and opportunities. Equipment can now be made available to public schools, and coordination and support is being provided to facilitate this process. The program strongly emphasizes providing handson summer research opportunities to underrepresented minority students at the high school level. LBL has developed a four week summer science experience for students at the Lab. Student selection is based on demonstrated potential and references by teachers. In 1993 six students worked at LBL and were paid through use of Department of Labor programs targeted at the eccnomically disadvantaged.

The Partnership for Environmental Technology Education (PETE) concentrates on linking the DOE Laboratories' resources with California Community Colleges. Carried out with the Lawrence Livermore National Laboratory, PETE provides support and professional development to 
The Allany High School 199.3 National Science Bowl Champions pose with their trophy. $L B L$ sponsored the regional science bowl that qualified Albany for the national championship. LBL's Associate Director-at-Large Glenn Seaborg and Deputy Director Pier Oddone were on hand to welcome the team back irom Washington.

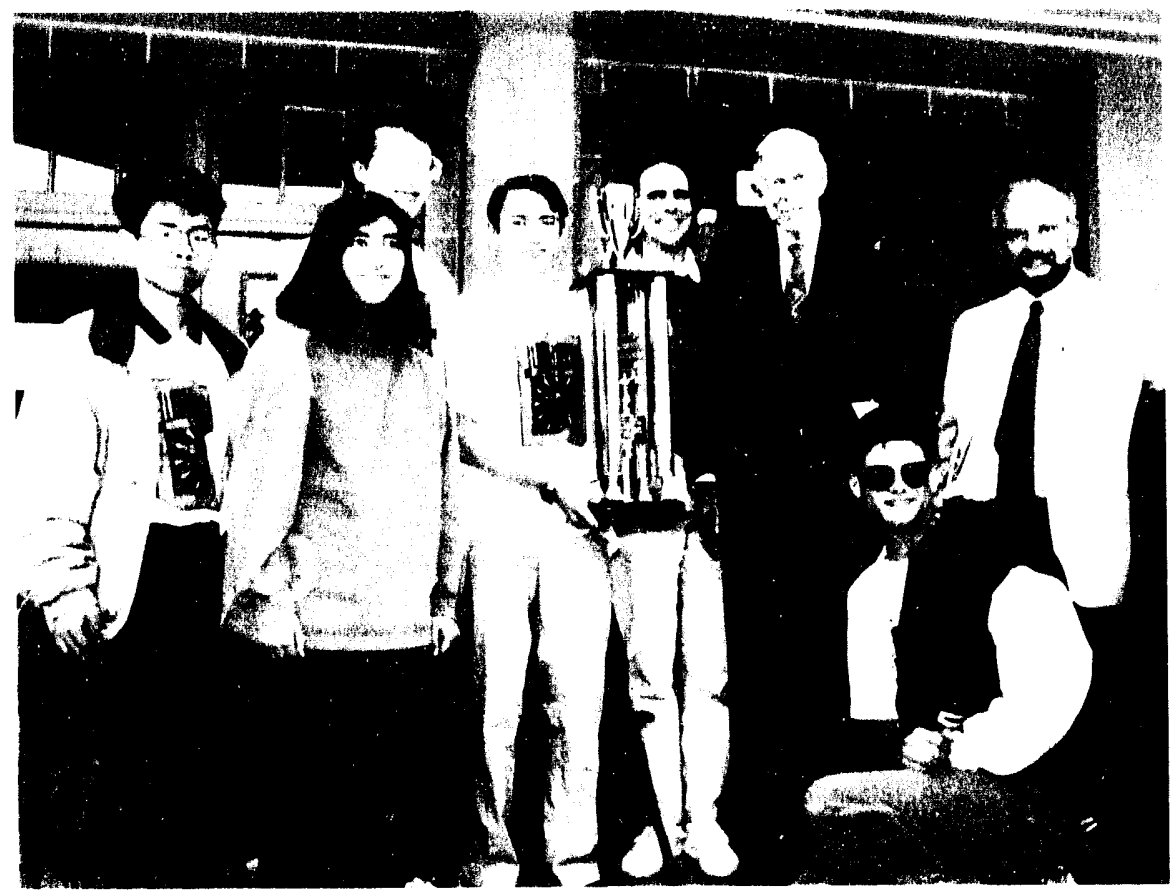

community college faculty for preparing students for careers in environmental management. LBL. plans to expand its activities with the local community colleges to create cooperative research and education centers. These centers will support collaborative research activities with the faculty of the community college, allowing them to use this base of activity for educating and retraining the reentry and largely minority student populations in science and technology.

\section{Technology in the Curriculum-The Hands-On Universe (HOU) Project}

The Hands-On Universe (HOU) project provides the means to transfer the technology, tools, and methods of inquiry of LBL's Astrophysics Group, worldleaders in automated supernova searches, to high school science classrooms, science museums and community based technology centers across the United States. HOU integrates real-time astronomical images, contemporary astrophysics research, and newly emerged technologies, to teach the processes of science and develop relevant scientific curriculum materials for use in high school classrooms and science and technology centers.

With support from the National Science Foundation, the HOU program is being transferred to the public science arena through the collaborative efforts of the HOU staff, the Boston Museum of Science, the Macon Museum of Arts and Sciences, and the Association of Science and Technology Centers. The HOU staff at LBL and museum partners are constructing and testing a model which uses science museums and lechnology centers as reglonal dissemination nodes for the HOU project. These "nodes" would distribute materials, train teachers, and serve as electronic network connection points for HOU participant school and the community at large. The partnership will also create museum-based programs and exhibits based on the HOU project. 
Most HOU program development to date has been in the secondary school area. Over the past two years LBL has assembled a group of 35 teachers and have trained them to use image processing and other state-ofthe-art technology to do astrophysics research. Working with these teachers, HOU scientists, teachers, and students have developed curricula materials for use with specific astrophysics group data sets.

During this period HOU program has run two very successful summer teacher workshops. The purpose of these workshops has been to evaluate materials and to train teachers to use modern image processing technology. The first workshop coincided with the July, 1991 total solar eclipse in Hawaii. With the support of the DOE Office of University and Science Education, the LBL Physics Division, IBM and Chevron, 12 teachers, a few LBL scientists with 3 CCD camera systems and a half dozen PC's donated by IBM convened in Hawaii to train high school teachers to use PCVista and other image processing tools, and to view the eclipse and acquire high quality digital images. One of these images, taken at Hapuna Beach, Hawaii, by American Fork High School teacher Curtis Craig, was selected for the cover of the DOE Education catalogue.

Inherent in the HOU structure is an evaluation mechanism: new materials are introduced and tested at HOU summer evaluation and teacher training workshops, where they are critiqued by teacher participants. Similar evaluations are collected during classroom trials of the HOU materials. Feedback obtained from these evaluation efforts will dictate refinements to the materials as they are being created.

\section{The Traditional Role of LBL in Undergraduate, Graduate, and Postgraduate Education - Partnerships Ensure Diversity}

LBL will continue to be an education and training center for students and faculty from the undergraduate to postdoctoral level. Over 300 undergraduate students from across the country, as well as the Berkeley Campus work as research assistants from eight weeks to six inonihs each year. These students join the research and engineering teams at the Laboratory and are assigned projects and responsibilities to support team goals. They attend seminars, lectures and conferences at the Laboratory and are expected to write technical reports and give technical presentations on their work. Over 400 graduate students and 200 postdocs are employed at LBL annually. Through the outreach efforts of the Center for Science and Engineering Education and minority partnerships in engineering and health and safety, over 100 students were from underrepresented minority populations. Partnerships with the California State University System provided opportunities for approximately 12 minority students through the Minority Access to Energy Research Careers program. Over 25 students were supported under the LBL/ISU/AGMUS Science Consortium Program described above. LBL also provides support to the 10 students through the Environmental Career Opportunities for Minorities program, an alliance of 15 Historically Black Colleges and Universities and Minority Institutions. At LBL students have a chance to experience a program with a diverse student population. 
Science/Math Educational Program Participation

\begin{tabular}{|c|c|c|c|c|c|c|}
\hline \multirow[b]{2}{*}{ Program } & \multicolumn{3}{|c|}{ FY 1992} & \multicolumn{3}{|c|}{ FY 1993} \\
\hline & Total & Minorities & Women & Totals & Minorities & Women \\
\hline \multicolumn{7}{|l|}{ Precollege Programs } \\
\hline $\begin{array}{l}\text { Bay Area Science and Technology } \\
\text { Education Collaboration (Teachers) }\end{array}$ & 700 & 280 & 490 & 908 & 411 & 621 \\
\hline $\begin{array}{l}\text { Bay Area Science and Technology } \\
\text { Education Consortium (Students) }\end{array}$ & 5600 & 4130 & 3920 & 4215 & 3837 & 2078 \\
\hline $\begin{array}{l}\text { Teacher Research Associates } \\
\text { (Teachers) }\end{array}$ & 42 & 11 & 23 & 44 & 7 & 21 \\
\hline $\begin{array}{l}\text { High School Honors Program } \\
\text { Life Sciences (Students) }\end{array}$ & 64 & 8 & 35 & 64 & 12 & 35 \\
\hline $\begin{array}{l}\text { High School Science } \\
\text { Symposium (Students) }\end{array}$ & 275 & 55 & 100 & $97^{a}$ & 27 & 58 \\
\hline $\begin{array}{l}\text { Updating Science Knowledge } \\
\text { for Instruction (Teachers) }\end{array}$ & 400 & 100 & 180 & 450 & 75 & 208 \\
\hline Science Bowl (Students) & 80 & 5 & 5 & 80 & 10 & 12 \\
\hline $\begin{array}{l}\text { Student Research Assistantships } \\
\text { (Division Hires) }\end{array}$ & 200 & 50 & 90 & 200 & 50 & 90 \\
\hline \multicolumn{7}{|l|}{ Undergraduate Programs } \\
\hline Community College Transfer & 5 & 2 & 2 & 5 & 4 & 3 \\
\hline Laboratory Co-op Program & 20 & 5 & 9 & 5 & 1 & 2 \\
\hline $\begin{array}{l}\text { Environmental Management Career } \\
\text { Opportunities for Minorities }\end{array}$ & 5 & 5 & 2 & 3 & 3 & 3 \\
\hline $\begin{array}{l}\text { Minority Access to Energy } \\
\text { Research Careers }\end{array}$ & 8 & 6 & 4 & 11 & 10 & 4 \\
\hline LBL/JSU/AGMEF Science Consortium & 27 & 26 & 7 & 24 & 23 & 15 \\
\hline $\begin{array}{l}\text { Science and Engineering } \\
\text { Research Semester }\end{array}$ & 37 & 2 & 20 & 28 & 2 & 10 \\
\hline Faculty/Student Teams (Faculty) & 5 & 4 & 1 & 2 & 2 & 1 \\
\hline
\end{tabular}

Minority numbers are for underserved groups only.

"Does not include Southern California Symposium, provided in 1992.

Divisions within LBL have also made significant efforts in outreach to students. The Engineering Division has two programs related to engineering education. The first is a corporate membership in the National Consortium for Graduate Degrees for Minorities in Engineering and Science, Inc. This will provide summer internships for students between their undergraduate degree and the first and second years of graduate school (two summers for 
each student). The second Engineering Division program is a six month coop internship for outstanding students with an emphasis on minority students. Both these programs are directed at providing a proven source of minority career employees. The Accelerator and Fusion Research Division has placed students at the Advanced Light Source, while the Environment, Health and Safety and Physics Divisions have placed students in their programs as well. The Engineering Division and Environmental Health and Safety Division provided support to a total of 8 students from Florida A\&M University's "Life Gets Better Program." In another program, the LBLproduced chart "Standard Model of Fundamental Particles and Interactions" has become an internationally recognized educational tool.

\section{The Future Role of LBL - Students and Teachers as Partners in Science and Technology}

The emphasis in science education for the future must be on providing students with the technical skills and knowledge they will need to be creative problems solvers, effective managers of information, and team players in the fields of science and technology. The challenge is to do this equitably and on a larger scale than has been previously done, ensuring that all students, irrespective of race, gender, and economic circumstances, have the chance to participate in scientific endeavors. I.BL through its Center for Science and Engineering Education will continue to meet this challenge through strategic partnerships such as those described above and through the use of high performance computing and the National Information Infrastructure to allow students and teachers access to the world of science.

Educational Programs Resource Requirements ${ }^{\mathrm{a}}$

\begin{tabular}{lrrrrrr}
\hline Category & 1994 & 1995 & 1996 & 1997 & 1998 & 1999 \\
\hline Budget & 3.5 & 3.5 & 3.5 & 3.5 & 3.5 & 3.5 \\
Staff (FTE) & 15 & 15 & 15 & 15 & 15 & 15 \\
\hline
\end{tabular}

"Projected funding through the Office of University and Science Education Programs ( $B \& R$ codes $K V$ and $K T$ ). 


\title{
8 HUMAN RESOURCES
}

\author{
Commit to developing our people to their fullest potential. \\ We value and seek diversity in our work force. We will \\ create an environment that respects the individual, \\ encourages leadership, stimulates innovation, fosters \\ integrity, and demands excellence.
}

LBL Vision 2000

\section{LABORATORY PERSONNEL}

LBL's most valuable resource is its people-the scientists, engineers, and support staff who contribute their many diverse skills to advance the Laboratory's research programs. The Laboratory's scientific and engineering staff are known for a wide range of accomplishments and honors. Nine LBL scientists have become Nobel laureates, sixteen have won Lawrence Awards, and four have won Fermi Awards. Of its present staff, 55 have been elected to the National Academies of Sciences and Engineering. Much of this success is founded on the Laboratory's ability to create highly effective teams of scientists, engineers, technicians, and students-then to orchestrate their efforts to produce a rich yield of basic knowledge and applied technology. From scientific leadership to technical expertise to administrative support-all parts of the team are necessary if we are to succeed.

A large part of the Laboratory's success is also owed to the many graduate and undergraduate students who contribute their efforts each year, as well as the many senior staff scientists jointly appointed as faculty on the $\cup C$ campuses, primarily UC Berkeley. This relationship with $U C$ provides a unique ability to interact with the broader university community, and helps to attract and retain a professional staff of high caliber. All of these factors contribute to LBL's mission to promote excellence in education and training, 
both for its own employees and for the greater scientific community. Our ultimate goal is to offer exceptional opportunities for professional growth, in an environment where achieveme. $1 t$ is recognized and rewarded at every level.

Laboratory Staff Composition (Full- and Part-Time Personnel-FY 1992)

\begin{tabular}{lccccc}
\hline Group & Doctoral & Master's & Bachelor & Other & Total \\
\hline Professional Staff & & & & & \\
Staff Scientists & 648 & 73 & 73 & 18 & 812 \\
& $(18.0)$ & $(2.0)$ & $(2.0)$ & $(0.5)$ & $(22.5)$ \\
Engineers & 152 & 119 & 97 & 21 & 389 \\
& $(4.2)$ & $(3.3)$ & $(2.7)$ & $(0.6)$ & $(10.8)$ \\
ManagementAdministrative & 30 & 86 & 204 & 332 & 652 \\
& $(0.8)$ & $(2.4)$ & $(5.7)$ & $(4.2)$ & $(18.1)$ \\
Suppont Staff & & & & & \\
Technicians & 18 & 79 & 181 & 799 & 1077 \\
& $(0.5)$ & $(2.2)$ & $(5.0)$ & $(22.2)$ & $(29.9)$ \\
All Other & 18 & 106 & 376 & 177 & 677 \\
& $(0.5)$ & $(2.9)$ & $(10.4)$ & $(4.9)$ & $(18.8)$ \\
Total & 866 & 463 & 931 & 1347 & 3607 \\
& $(24.0)$ & $(12.8)$ & $(25.8)$ & $(37.3)$ & $(100.0)$ \\
\hline
\end{tabular}

\section{Recruitment Program}

To prepare for the new challenges that lie ahead, we must not only maintain our foundation of excellence, we must build on it. Since its beginning, the Laboratory has had a strong commitment to train the next generation of scientists and engineers. The education programs of the Center for Science and Engineering Education are instrumental in encouraging young people, especially women and minorities, to enter careers in science and engineering disciplines, and to better position them to attain their career goals. LBI. is now exploring ways to maintain contact with these students after they have completed a Laboratory program and as they progress through the educational system in order to continue to encourage and assist them in achieving their goals.

In an effort to retain young staff as well as to recruit promising scientists to the Laboratory, LBL is currently restructuring its scientist and engineer classification and pay program so that it will provide more clearly defined career paths in the areas of both scientific accomplishment and scientific management. The Laboratory is directing its recruitment program toward three goals: (1) ensuring a breadth of experience, (2) maintaining a strong scientific and technical base in the work force, and (3) committing to its affirmative action, equal opportunity goals. LBL is active in recruiting promising scientists and engineers through its divisional fellow and postdoctoral associate programs, and each division is accountable for affirmative action/equal employment opportunity (AAEEO) action-oriented programs aimed at achieving a diverse work force. LBL has effected the following specific steps to achieve these goals: 
- A program to increase the Laboratory's comperitiveness in the recruitment marketplace, including an active advertising campaign, strong representation at job fairs, and training programs.

- Other outreach recruitment programs, including a widely distributed job listing, professional seminars, and search commiltees, including extensive participation of managers and supervisors.

- Special employment and internship programs to increase employment opportunities, including summer, student, and youth employment programs, as well as minority education programs.

\section{PERSONNEL DEVELOPMENT PROGRAMS}

As part of its continuing effort to improve its staff capabilities, to establish standards of safety excellence, and to increase opportunities for women and minorities, the Laboratory supports and conducts a number of professional development programs, coordinated by the Laboratory's Employee Development and Training Office. More than 30 development courses and seminars are offered on site each year. The Laboratory also provides support for off-site training and education, including baccalaureate and advanced degrees as well as other professional training credentials. Scheduled on-site training and programs are conducted in environmental safety and health and in management, personnel, computer, and workstation skills.

The Development and Training Office informs employees of the resources available to them and encourages them to establish a formal Employee Development Plan in consultation with their supervisors. It also conducts special management institutes to improve employee performance and to enhance overall Laboratory management goals.

In addition to training, the Laboratory maintains an Employee Assistance Program (EAP), which offers several employee programs to promote retention, personal well-being, and effective job performance. These resources and programs are available through the Personnel Department Employee Relations Group and the Health Services Department. The program includes on-site consulting services for emotional problems and substance abuse, and off-site referral services. In addition, a new substance abuse training program has been implemented for supervisors and managers.

\section{Training and Development Programs}

Through a combination of on-the-job training, in-house training programs, and attendance at programs/courses provided by non-Laboratory institutions, the lechnical skills of the Laboratory's employees are constantly upgraded to meet the ever-changing needs brought about by rapid technological advances. Communication and training are the keys to achieving our diversity goals. For any program to be successful, it must be integrated at all levels of authority and supervision; therefore, a diversity training program is mandatory for all manag. ers and supervisors. This program not only helps them to understand cultural differences and manage diversily, but also solicits their assistance in creating a diverse work force at the Laboratory. In addition, it is the Laboratory's policy that all managers and supervisors be held accountable for achieving diversity goals, and as such they are reviewed annually to evaluate their performance with regard to AAVEO policies. 
In developing the leadership skills required to achieve the Laboratory's goals and objectives, an intensive training program for managers and supervisors has been developed. A pilot program has begun that includes approximately 90 managers and supervisors, Laboratory Heads, and Divi. sion Directors. II is anticipaled that all supervisors and managers will eventually participate, and the training will be integrated as part of the Laboratory's required training program.

In addition, the Human Resources Department offers a range of inan. agement and supervisory development courses. One of these is the ongoing Iraining program to assist managers and supervisors of disabled employees. Special events have been held to heighten the awareness of the Laboratory community regarding employment of the disabled, including workshops and demonstrations of equipment to assist disabled employees. The Vocational Rehabilitation Service is available to assist supervisors in making accommodations for employees and potential employees with disabilities. Other important I luman Resources Department programs and activities include on-site and off-site programs to assist employees in developing their skills. Several of these courses emphasize communications, including The Pronounced Difference: Speech Evaluation Workshop for Non-native Speakers of English. In addition, the Laboratory has an education assistance and luition reimbursement program to assist employees in obtaining advanced Iraining and education through approved employee development plans.

\section{Employee Services}

Other programs at LBL offer the following services:

- The Health Services Department at LBL provides a range of health services, including physical examinations, evaluations for return-10-work, eye examinations for laser users, a bioassay program for employees who work with radioactive substances, and a blood bank donation program.

- The Employees Recreation Association, an LBL recreational and cultural organization that includes all LBL. employees and their imme. diate families, supports existing and future special-interest clubs, and offers various other discount opportunities for employees.

- The Vocational Rehabilitation Program provides counseling, vocational evaluation, job restrucluring, and worksite modification. The Vocational Rehabilitation Coordinator is available to all employees.

- The flextime policy at LBL provides some flexibility for scheduling employee work hours, consistent with meeting all operational requirements of divisions and departments. It facilitates commuting, childcare activities, and other scheduling needs of employees.

- In FY 1992 the Laboratory conducted a survey to obtain information from LBL employees regarding their dependent-care responsibilities; the results will be used to lay the groundwork for programs to address work and family issues. The Employee Assistance Program has also held a childe are and eldercare information fair for employees and has distributed handbooks on meeting childcare and eldercare responsibilities. The elderc are guide has become a model for use in the UC system and has been distributed to other national laboratories.

An Employee Handbook (PUB 80) with an extensive description of programs, benefits, and personnel services is provided to employees. 


\section{WORK FORCE DIVERSITY}

One of the guidling princ iples of Vision 2000 is a reaffirmaltion of our commitment to work force diversity 10 . c cultural ethic thall fully respeects every individual. The Labordlory is commifted to AA and EE( ) in all aspects of employment. This means integrating diversity into its research culture and providing an environment that is accessible, equitable, and hospitable 16 all employees.

The Laboratory's commitment, coupled with the recognition of the changing dymamics of the work force and increasing competition among the scientific and technological industries, means that we must work harder to make Lawrence Berkeley Laboratory an employer of choice. To achieve this goal, the Laboratory has formed a Work Force Diversity Office and Commit. tee on Diversity as well as initiated a wide range of programs that will enhance our ability to attract and retain qualified individuals. We recognize that there is still much to be done to accomplish this goal. What follows is a sampling of our efforts.

\section{Work Force Diversity Office}

In lanuary 1993, the Director established the Work Force Diversity Office, which subsumed the former Office of Equal Opportunity and its affirmative action functions. The establishment of this Office will advance the Laboratory from being a compliance entity to becoming an institution that incorporates proactive programs addressing the broad issues of work force diversity. As part of its charter, the Work Force Diversity Office will focus and direct senior management toward developing and implementing a broad-based strategy targeted at diversifying the work force, managing diversity issues, coordinating recruitment and outreach efforts, and enhanc. ing employee development practices. The Head of the Work Force Diversity (Office reports direcily to the Laboratory Director, works closely with the Deputy Laboratory Director on related aspects of strategic planning, and is management's liasison to the Committee on Diversity to facilitate implementation of the commiltee's formal recommendations.

The Work Force Diversity Office, in partnership with Laboratory management, will undertake the following specific programmatic initiatives:

- Aggressively seek women, people of coles. and individuals from other protected classes who have the potential to achieve excellence at LBL. Initiate new recruitment methods that identify potential candidates early in their educational careers.

- Mentor employees so that they can achieve excellence.

- Ensure a working atmosphere that is supportive and gives a sense of belonging to employees from all culleures.

- Provide resources 60 help managers and supervisors implement the Laboratory's alfirmative action program.

- Develop new capabilities for the Laboratory and each division lo review its performance with regard to affirmative action issues.

\section{Committee on Diversity}

The Commitlee on Diversity was constituted by the Direc tor of the Laboralory in lanuary 1992. The commiltee is composed of a representative from each division who serves a three-year ferm. The members selededed are 
representative of the different classification levels at the Laboratory, from the clerical support level up to the scientific level. Committee members are placed on one of three subcommiltees and focus on issues related to fecruit. ment, laboratory environment, and education. The subrommiltees meet and consider topics related to areas of responsibilities and convene with the full committee to make its recommendations to the Director. Issues to be addressed by the committee can best be summed by L.BL's Statement or: Diversity that reads as follows:

$L B L$ is an institution with a tradition of, and dedication to, excellence in scientific research, lechnological innovaltion, educational opportunities and service to the nattion. Reflecting the nation's values, $L B L$ is dedi. cated to integrating diversity into its research culture and to providing an environment that is accessible and hospitable to all employees.

\section{Affirmative Action Programs}

While an affirmative action program is required by federal regulations, the Laboratory considers affirmative action as a mechanism to promote diversity. The following is a representation of several programmatic achievements that occurred in 1992 and 1993:

Managing Diversity Workshops. Throughout last year, the Laboratory continued to offer a mandatory workshop for supervisors and managers that covered issues pertaining to management in an increasingly diverse workplace. To date, this training has reached over 90 percent of the Laboratory's supervisors and managers.

Community Relations Office. The Community Relations Office, begun in lanuary of 1992, is helping LBL to strengthen its good-neighbor standing in the surrounding communities, to find new opportunities for creating awareness of LBL in the Bay Area, and to provide information and guided tours for the general public.

Culture of Women and Science. In June 1992, the Laboratory joined with the National Science Foundation and the University of California at Berkeley in sponsoring a three-day workshop entitled, The Changing Culture in Science-Bringing it into Balance. Approximately 100 participants from across the United States convened in Berkeley to develop strategies for accelerating positive change in the environment of our schools and laboratories in order to improve, in particular, the integration of women, people of color, and individuals from other protected classes.

Dependent Care Survey. In July 1992, the Laboratory conducted a survey to determine employee needs in terms of child care and adult care responsibilities, the impact of these responsibilities on employees' work lives, and to seek suggestions for programs and benefits that would support employees. Approximately 75 percent of the employees responded to the survey, with almost half indic ating they had either child care or adult care responsibilities. The results of the survey are now under review by Laboratory management.

Organization for Women Scientists and Engineers. In August of last year, a group of women scientists and engineers formed an organization aimed at creating a forum to meet and discuss one another's research as well as mutual areas of professional concern. The group has been meeting on a monthly basis. 
Educational Outreach Program. In Septermber 1992, the Center for Science and Engineering Education introduced an educational outreach program that makes Laboralory staff av.rilable (1) assist teachers and students in Bay Area schools. Scientists, engineers, graduate students, administrators, managers, lechnical and suppont staff participale in the program. LBL's Education Outreach Coordinator has matched volunteers as role models/mentors, lutors, lecturers, and resource specialists to classroom leachers.

Outstanding Performance Awards. In October 1992, the Laboratory introduced an Outstanding Performance Award program. Among the criteria for selection was contribution to outstanding techniques or procedures, technical innovation or notable accomplishment in improving environmental, health or safety conditions at the Laboratory, or demonstrated leadership and success in employee development, affirmative actions, diversified work force and communily relations. The monetary awards range from $\$ 1,000$ to $\$ 5,000$.

Training Program for Managers and Employees. In addition to a full complement of supervisor training, the Work Force Diversity Office introduced two new courses focusing on the topic of sexual harassment. A class for supervisors and managers, entitled Responding to Complaints of Sexual Harassment: A Supervisor's Responsibility, provides information to better understand the law and to provide guidance to supervisors and managers on responding to complaints. Over 200 supervisors and managers attended classes held between July and September of 1992. The class for employees, entitled Sexual Harassment: Employee Rights and Responsibilities, provides information on the law and on employee's rights and responsibilities as they relate to sexual harassment. Over 120 employees attended classes held between July and September of 1992. Both classes will continue to be offered through 1993.

Implementation of Americans with Disabilities Act. In response to the recent requirements of the Americans with Disabilities $A C I$ (ADA), the Laboratory developed and implemented guidelines and informational materials for the Laboratory's community. Representatives from the Laboratory's Employee Assistance Program, Risk Management, Employment/ Staffing Office. Human Resources Department's Policy and Benefits Unit, Facilities Department, and the Office of Equal Opportunity provided resources to accomplish this effort. Since the enactment of the new requirements of the Act, the Laboratory has distributed informational materials to all supervisors and managers and offered training sessions to all employees about the requirements of the Act. An ADA Handbook has been developed and is available through the Human Resources Department.

Commitment to People Task Force. In November 1992, the Laboratory convened a lask force with a charge to identify steps the Laboratory may take to improve the quality of work life at I.BL. The task force's recommendations will be formulated and reviewed by management in 1993.

Management Skills Assessment Program. Originally designed to provide career development support for women, people of color, and individuals from other protected classes, participants gain a better understanding of their strengths and an action plan for continued development of their management skills. Participants also gain an improved understanding of the supervisory role by developing skills to give effective feedback to direct reports. 
In March 1993, the Laboratory sent four employees to participate in the program and plans on sending four employees to each yearly session.

\section{University of California Los Angeles/Leadership Educalion for Asian Pacifics (UCLA/LEAP) Technology and Aerospace Management Program.} Leadership Eclucation for Asian Pacifics, Inc, is a nonprofit, educational, community-based organization that sponsors the UCLALEAP Technology and Aerospace Management Program. This one-week residential program for Asian Pacific American technical and administrative managers is designed to address issues related to strategic management, stereotypes, self. assessments in principles related to str. 'ejic management atid organizational design in high technology and aerospace, self-assessment of strengths in their organization, performance in dynamic group settings and in formal presentations, and career planning skills. The program was introduced to the Laboratory in 1992 and participation is on a division-by-division basis. To date, the Laboratory sent three employees to participate in the program and will continue its involvement in upcoming sessions.

UC President's Postdoctoral Fellowship Program. The Regents established this program in order to improve the quality and diversity of UC faculty and to enhance the competitiveness of outstanding people of color and women Ph.D. degree holders for appointments at UC campuses and the DOE laboratories. The Laboratory will host two fellows annually and each fellowship is for a 12 month period, renewable for a second year pending evidence of satisfactory progress. Two posidoctoral fellows are currently in the program.

\section{AFFIRMATIVE ACTION 1993}

In 1993, the Laboratory will undertake a series of new efforts that will focus on areas of recruitment, promotion, and retention. These efforts will augment others already in place, such as those discussed earlier. In proceeding, the Work Force Diversity Office will foster collaboration among the divisions with various Laboratory administrative functions to develop programs and activities for supervisors and managers accountable for meeting our affirmative action goals.

In this year's recruitment efforts, the Work Force Diversity Office is working with the Human Resources Department and the Public Information Department to create recruitment materials to enhance the image of the Laboratory and a Recruitment Resource Guide that identifies organizations, networks, and contacts to broaden recruitment sources when vacant positions are being filled.

In addition to these efforts, the Work Force Diversity Office and the Human Resources Department participate in outreach recruitment activities in order to attract and recruit women, people of color, and individuals from other protected classes to the Laboratory in all classifications. Specific activities have included participation in the Public Health Carrier Day at the University of California at Berkeley, the Chinese for Affirmative Action Job Fair, the Bay Area Urban League Job Fair, the Rio Grande Research Corridor for High Tech Minority and Women Job Fair, and the Conference of Minority Public Administrator foh Fair.

A new component to the recruitment campaign is the Human Resources Department's computerized screening system (RESUMIX). The Work Force Diversity Office will work with the Human Resources Department to 
develop a Resume Bank that will store the resumes of women and minorities for use by the divisions and the Human Resources Department when filling vacancies throughout the year.

In the area of promotion and retention, the Work Force Diversity Office coordinates the Laboratory's participation in two residential programs for women, people of color, and individuals from other protected classes. The first program, the Management Skills Assessment Program, is a four-day career development program administered by the University of California. Each division is given an opportunity to nominate individuals from underrepresented groups and other protected classes to participate in the program. The Laboratory will send four employees per year to the program, and the Work Force Diversity Office will coordinate the nomination process with all divisions.

The second program, the UCLA/LEAP Technology and Aerospace Management Program, is a five-day development program hosted in Southern California. Selection of the Laboratory's Asian Pacific employees is on a division-by-division basis. Within the past year of participation, the Laboratory sent three employees, and evaluations have been very positive.

A critical component to the promotion process is the evaluation of an employee's performance. The Work Force Diversity Office will survey other organizations to identify training and development efforts that have effectively responded to meet the needs of women, people of color, and individuals from other protected classes in the work force. The goal of this effort will be development of training programs to assist these groups in becoming more competitive in applying for positions that offer advancement. In addition, each division will collaborate with the Human Resources Department in developing an improved system for evaluating employee performance. A major component of that system will be identification of strong areas of performance, areas in which performance can be strengthened, and development of a plan for the employee's career growth.

\section{Affirmative Action Plans}

On an annual basis, the Laboratory prepares two Affirmative Action Plans, which are approved by the University of California and provided to the U.S. Department of Energy. The Equal Opportunity Administrator is responsible for the audit and implementation of the Laboratory's affirmative action program. The Affirmative Action Plans allow the Laboratory to establish and audit its affirmative action and equal employment opportunities activities and performance. The first plan focuses on women and underrepresented groups, and the second plan focuses on Vietnam-Era Veterans and persons with disabilities. These plans are distributed to each of the Laboratory's 12 divisions, senior-level management offices, and the Laboratory's five on-site libraries. Copies are available to employees, applicants, and compliance agencies upon request through the Work Force Diversity Office. The plans provide the Laboratory with an opportunity to discuss analyses of the Laboratory's underutilization and division-based adverse impact as well as Laboratory-wide and division-based affirmative action efforts of the preceding year. On an annual basis, the Equal Opportunity Administrator meets with the Laboratory Director, Deputy Laboratory Director, Associate Laboratory Directors, and each Division Director to discuss the plar,s in detail.

The following two tables are excerpted from the Laboratory's 1988 and 1993 Affirmative Action Plans. Data source for each plan year is based on the preceding fiscal year (October 1 through September 30). The two tables 
show Laboratory population for the 1987 and 1992 fiscal years and provide a breakdown by Federal occupation category and by race. Population figures are now based on career-status employees. Despite the shrinking availability pool, (particularly at the PH.D. level) in the representation of women and underrepresented groups, the Laboratory population has remained relatively constant during this period. To increase the recruitment pool of qualified individuals, the Work Force Diversity Office will undertake various programs and initiatives, such as those mentioned earlier, that focus on staff career development as well as outreach recruitment to offset the dearth of women and underrepresented groups in the pipeline.

In preparation of the 1993 Affirmative Action Plan, the Work Force Diversity Office reviewed all job groups and, where necessary, restructured them to depict a more accurate representation of the classifications currently at the Laboratory. The Laboratory's present reporting mechanism is based on requirements dictated by the U.S. Department of Labor. As such, their specifications do not require a breakdown of the ethnic categories by gender. The Work Force Diversity Office is in the process of modifying the reporting procedure to include such a breakdown for future Institutional Plans.

Equal Employment Opportunity - 1987

\begin{tabular}{|c|c|c|c|c|c|c|c|c|}
\hline \multirow{3}{*}{$\begin{array}{l}\text { Federal Occupational Category } \\
\text { Gender }\end{array}$} & & & \multirow{3}{*}{$\begin{array}{l}\text { Total } \\
\text { White }\end{array}$} & \multicolumn{5}{|c|}{ Minority } \\
\hline & \multicolumn{2}{|c|}{ Total } & & \multirow{2}{*}{$\begin{array}{l}\text { Total } \\
\text { M\&F }\end{array}$} & \multirow{2}{*}{$\begin{array}{l}\text { Black } \\
\text { M\&F }\end{array}$} & \multirow{2}{*}{$\begin{array}{c}\text { Hispanic } \\
\text { M\&F }\end{array}$} & \multirow{2}{*}{$\begin{array}{l}\text { Asian } \\
\text { M\&F }\end{array}$} & \multirow{2}{*}{$\begin{array}{l}\text { Nat. Am. } \\
\text { M\&F }\end{array}$} \\
\hline & Male & Female & & & & & & \\
\hline OFFICIALS \& MANAGERS & $\begin{array}{c}95 \\
(82.61 \%)\end{array}$ & $\begin{array}{c}20 \\
(17.39 \%)\end{array}$ & $\begin{array}{c}105 \\
(91.30 \%)\end{array}$ & $\begin{array}{c}10 \\
(8.70 \%)\end{array}$ & $\begin{array}{c}7 \\
(6.09 \%)\end{array}$ & $\begin{array}{c}2 \\
(1.74 \%)\end{array}$ & $\begin{array}{c}1 \\
(0.87 \%)\end{array}$ & $\begin{array}{c}0 \\
(0.00 \%)\end{array}$ \\
\hline \multicolumn{9}{|l|}{ PROFESSIONALS } \\
\hline \multirow[t]{2}{*}{ Scientists \& Engineers } & 757 & 130 & 755 & 132 & 18 & 13 & 100 & 1 \\
\hline & $(85.34 \%)$ & $(14.66 \%)$ & $(85.12 \%)$ & $(14.88 \%)$ & $(2.03 \%)$ & $(1.47 \%)$ & $(11.27 \%)$ & $(0.11 \%)$ \\
\hline \multirow{2}{*}{ Management \& Administrative } & 51 & 105 & 114 & 42 & 16 & 11 & 15 & 0 \\
\hline & $(32.69 \%)$ & $(67.31 \%)$ & $(73.08 \%)$ & $(26.92 \%)$ & $(10.26 \%)$ & $(7.05 \%)$ & $(9.62 \%)$ & $(0.00 \%)$ \\
\hline \multirow[t]{2}{*}{ TECHNICIANS } & 276 & 57 & 242 & 91 & 28 & 19 & 41 & 3 \\
\hline & $(82.88 \%)$ & $(17.12 \%)$ & $(72.67 \%)$ & $(27.33 \%)$ & $(8.41 \%)$ & $(5.71 \%)$ & $(12.32 \%)$ & $(0.90 \%)$ \\
\hline \multicolumn{9}{|l|}{ All Other (Crafts, Laborers, Service } \\
\hline \multirow[t]{2}{*}{ Workers, Office, and Clericals) } & 318 & 256 & 360 & 214 & 107 & 58 & 43 & 6 \\
\hline & $(55.40 \%)$ & $(44.60 \%)$ & $(62.72 \%)$ & $(37.28 \%)$ & $(18.64 \%)$ & $(10.10 \%)$ & $(7.49 \%)$ & $(1.05 \%)$ \\
\hline Total All Categories & $\begin{array}{c}1497 \\
(72.49 \%)\end{array}$ & $\begin{array}{c}568 \\
(27.51 \%)\end{array}$ & $\begin{array}{c}1576 \\
(76.32 \%)\end{array}$ & $\begin{array}{c}489 \\
(23.68 \%)\end{array}$ & $\begin{array}{c}176 \\
(8.52 \%)\end{array}$ & $\begin{array}{c}103 \\
(4.99 \%)\end{array}$ & $\begin{array}{c}200 \\
(9.69 \%)\end{array}$ & $\begin{array}{c}10 \\
(0.48 \%)\end{array}$ \\
\hline
\end{tabular}

Source: 1988 LBL Affirmative Action Plan-Figures are based on fiscal year 1987 (9/30/86-10/01/87). 
Equal Employment Opportunity - 1992

\begin{tabular}{|c|c|c|c|c|c|c|c|c|}
\hline \multirow{3}{*}{$\begin{array}{l}\text { Federal Occupational Category } \\
\text { Gender }\end{array}$} & & & \multirow{3}{*}{$\begin{array}{l}\text { Total } \\
\text { White }\end{array}$} & \multicolumn{5}{|c|}{ Minority } \\
\hline & \multicolumn{2}{|c|}{ Total } & & \multirow{2}{*}{$\begin{array}{l}\text { Total } \\
\text { M\&F }\end{array}$} & \multirow{2}{*}{$\begin{array}{l}\text { Black } \\
\text { M\&F }\end{array}$} & \multirow{2}{*}{$\begin{array}{c}\text { Hispanic } \\
\text { M\&F }\end{array}$} & \multirow{2}{*}{$\begin{array}{l}\text { Asian } \\
M \& F\end{array}$} & \multirow{2}{*}{$\begin{array}{c}\text { Nat. Am. } \\
\text { M\&F }\end{array}$} \\
\hline & Male & Female & & & & & & \\
\hline OFFICIALS \& MANAGERS & $\begin{array}{c}119 \\
(78.81 \%)\end{array}$ & $\begin{array}{c}32 \\
(21.19 \%)\end{array}$ & $\begin{array}{c}131 \\
(86.75 \%)\end{array}$ & $\begin{array}{c}20 \\
(13.25 \%)\end{array}$ & $\begin{array}{c}6 \\
(3.97 \%)\end{array}$ & $\begin{array}{c}6 \\
(3.97 \%)\end{array}$ & $\begin{array}{c}8 \\
(5.30 \%)\end{array}$ & $\begin{array}{c}0 \\
(0.00 \%)\end{array}$ \\
\hline \multicolumn{9}{|l|}{ PROFESSIONALS } \\
\hline Scientists \& Engineers & $\begin{array}{c}770 \\
(81.40 \%)\end{array}$ & $\begin{array}{c}176 \\
(18.60 \%)\end{array}$ & $\begin{array}{c}743 \\
(78.54 \%)\end{array}$ & $\begin{array}{c}203 \\
(21.48 \%)\end{array}$ & $\begin{array}{c}16 \\
(1.69 \%)\end{array}$ & $\begin{array}{c}16 \\
(1.69 \%)\end{array}$ & $\begin{array}{c}170 \\
(17.97 \%)\end{array}$ & $\begin{array}{c}1 \\
(0.11 \%)\end{array}$ \\
\hline Management \& Administrative & $\begin{array}{c}65 \\
(29.68 \%)\end{array}$ & $\begin{array}{c}154 \\
(70.32 \%)\end{array}$ & $\begin{array}{c}162 \\
(73.97 \%)\end{array}$ & $\begin{array}{c}57 \\
(26.03 \%)\end{array}$ & $\begin{array}{c}18 \\
(8.22 \%)\end{array}$ & $\begin{array}{c}13 \\
(5.94 \%)\end{array}$ & $\begin{array}{c}26 \\
(11.87 \%)\end{array}$ & $\begin{array}{c}0 \\
(0.00 \%)\end{array}$ \\
\hline TECHNICIANS & $\begin{array}{c}434 \\
(88.03 \%)\end{array}$ & $\begin{array}{c}59 \\
(11.97 \%)\end{array}$ & $\begin{array}{c}379 \\
(76.88 \%)\end{array}$ & $\begin{array}{c}114 \\
(23.12 \%)\end{array}$ & $\begin{array}{c}30 \\
(6.09 \%)\end{array}$ & $\begin{array}{c}28 \\
(5.68 \%)\end{array}$ & $\begin{array}{c}55 \\
(11.16 \%)\end{array}$ & $\begin{array}{c}1 \\
(0.20 \%)\end{array}$ \\
\hline \multicolumn{9}{|l|}{ All Other (Crafts, Laborers, Service } \\
\hline Workers, Office, and Clericals) & $\begin{array}{c}266 \\
(49.26 \%)\end{array}$ & $\begin{array}{c}274 \\
(50.74 \%)\end{array}$ & $\begin{array}{c}319 \\
(59.07 \%)\end{array}$ & $\begin{array}{c}221 \\
(40.93 \%)\end{array}$ & $\begin{array}{c}113 \\
(20.93 \%)\end{array}$ & $\begin{array}{c}56 \\
(10.37 \%)\end{array}$ & $\begin{array}{c}46 \\
(8.52 \%)\end{array}$ & $\begin{array}{c}6 \\
(1.11 \%)\end{array}$ \\
\hline Total All Categories & $\begin{array}{c}1654 \\
(70.41 \%)\end{array}$ & $\begin{array}{c}695 \\
(29.59 \%)\end{array}$ & $\begin{array}{c}1734 \\
(73.82 \%)\end{array}$ & $\begin{array}{c}615 \\
\text { (26.18\%) }\end{array}$ & $\begin{array}{c}183 \\
(7.79 \%)\end{array}$ & $\begin{array}{c}119 \\
(5.07 \%)\end{array}$ & $\begin{array}{c}305 \\
(12.98 \%)\end{array}$ & $\begin{array}{c}8 \\
(0.34 \%)\end{array}$ \\
\hline
\end{tabular}

Source: 1993 LBL Affirmative Action Plan-Figures are based on fiscal year 1992 (9/30/91-10/01/92). 


\section{SITE AND FACILITIES}

Make $L B L$ the location of choice for facilities and programs.

LBL Vision 2000

Lawrence Berkeley Laboratory is located in the hills above the UC Berkeley campus, on a 54-hectare (134-acre) site overlooking the San Francisco Bay. Founded in 1931 by Ernest O. Lawrence, the Laboratory was moved to its present site in 1940. It is the oldest of the DOE national laboratories, and the only one located adjacent to a major university.

Over the past decades LBL has continued on a course of diversification that has brought it wide recognition in high-energy and nuclear physics, materials science, chemistry, life sciences, and energy conservation research. For LBL to continue its mission as a multidisciplinary national laboratory, it must address some critical issues and opportunities concerning site and facility improvement. The Laboratory strongly supports strategic planning activities that are now taking place as part of a national effort to restore and maintain the nation's scientific infrastructure, and has made these activities an integral part of its own site development process. In addition, LBL is continually vigilant about creating conditions that protect the LBL staff, the public, and the environment.

\section{SITE DEVELOPMENT PLANS}

Each year LBL prepares site development plans to provide analysis and policy guidance for the effective use and orderly development of land and facilities at LBL. This planning effort is critical to all of the Laboratory's programs; first, because facilities require rehabilitating to avert safety hazards, shutdowns, and failures, and, second to optimize use of the Laboratory's limited land and building resources. In addition to the site development plan, department-wide planning efforts, such as the Five-Year Non-Defense Facilities Modernization Plan and the Capital Asset Management Plan (CAMP) are integrated into the total site planning process. The objectives of the site planning effort are as follows: 
- Evaluate future mission projections and anticipate DOE national research facility needs.

- Ensure a safe and healthy workplace in full compliance with building and fire codes.

- Protect the environment and buffer activities to enhance adjacent land uses.

- Protect the national investment in valuable government-owned research and support assets.

- Consolidate research and support services through proper siting of new buildings and maintenance of functional units.

- Improve access and communications within and to the Laboratory.

- Promote energy conversion and cost reductions through efficient building design, location, operation, maintenance, parking, and transportation.

Resources to improve the Laboratory's facilities are provided through Multiprogram Energy Laboratory Facilities Support (MEL-FS), General Plant Projects (GPP), In-House Energy Management, and General Purpose Equipment (GPE). Adequate funding from these resources is a critical element of LBL's ability to provide DOE with an effective multiprogram Laboratory that can meet environmental and safety standards. A major effort is under way to provide conditions that meet accepted standards for LBL's environment, health, and safety programs, including providing adequate monitoring and sample processing laboratories, emergency command and response facilities, and sufficient space for on-site offices for industrial hygiene, environment, and other essential EH\&S staff (see Section 6).

LBL's facilities planning is coordinated through specific Laboratory management activities and DOE initiatives. The Site Development Plan and the Laboratory Integrated Facilities Plan have been updated for FY 1993 and will continue to be updated annually. LBL has established a prioritization framework for its multiprogram capital projects and incorporates a safety and environmental program that supports the Capital Asset Management Program. The Laboratory's ten-year In-House Energy Management Plan represents significant opportunities for cost savings. All proposed projects undergo NEPA and CEQA review procedures for full compliance with SEN 15, DOE Order 5440.IE, and UC guidelines. Institutional planning acts to couple site management planning activities closely to program planning and other strategic management processes. For FY 1993 a complete 20-year infrastructure program has been developed consistent with DOE guidelines and supporting the Laboratory's Vision 2000 strategic planning.

\section{SITE DESCRIPTION AND STATUS}

The first building on the LBL site was constructed in 1940. In FY 1993 the main site includes 81 permanent buildings and 110 trailers and temporary structures. Over $70 \%$ of the permanent buildings are over 25 years old (see figure below). The existing facilities and the currently funded construction at LBL constitute $183,000 \mathrm{gsm}$ (1.97 Mgsf) located on the main site, the UC campus, and leased off-site locations. The space distribution upon completion of current projects in FY 1993 is shown below. 
Age distribution of permanent mainsite buildings lexcluding proposed FY 1993 construction).

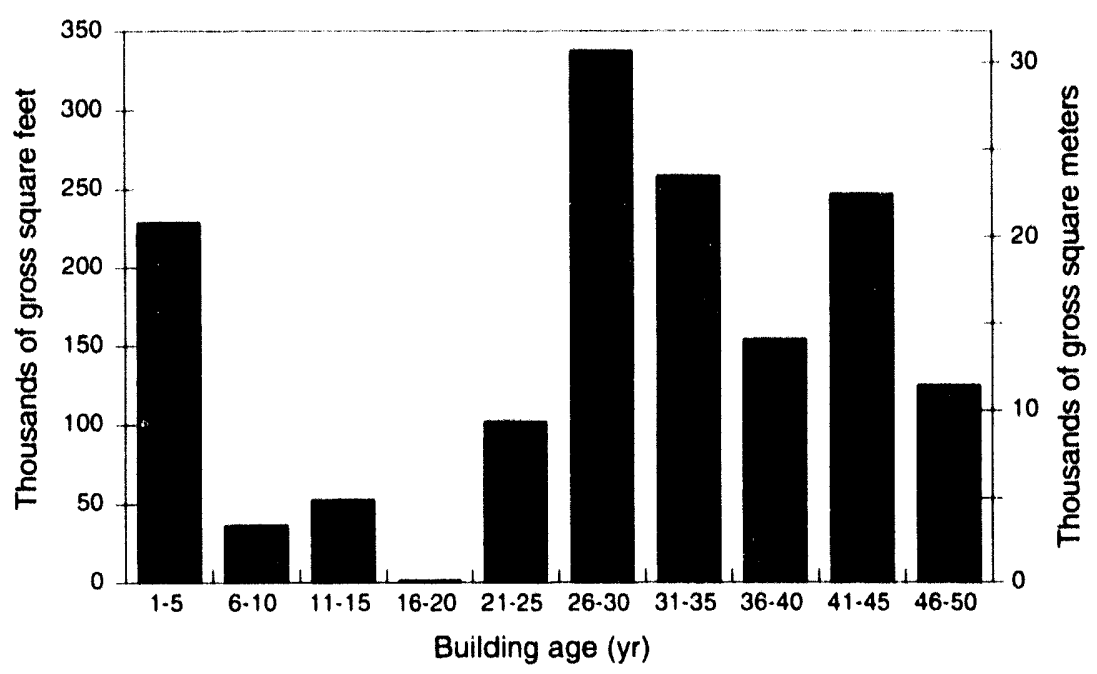

LBL Space Distributiond

\begin{tabular}{lccc}
\hline Location & Area $\left(1000 \mathrm{~s} \mathrm{~m}^{2}\right)$ & Area $(\mathrm{Mgs})$ & $\%$ of Total \\
\hline Main site & 153 & 1.65 & 83 \\
On campus and & 16 & 0.17 & 9 \\
$\quad$ Richmond Field Station & 14 & 0.15 & 8 \\
Off-site leased & 183 & 1.97 & 100 \\
Total & & &
\end{tabular}

Includes funded projects.

LBL space in campus buildings is available to DOE on a long-term arrangement. The off-site leased buildings provide space for essential research and support functions. Of the $153,000 \mathrm{gsm}(1.65 \mathrm{Mgsf})$ on the main site, about $6,630 \mathrm{gsm}(71,300 \mathrm{gsf})$ are in trailers and other temporary structures. The replacement value of the buildings, utilities, and other improvements at the main site, as determined by DOE's Real Property Inventory System for 1986, is more than $\$ 425 \mathrm{M}$ (see table following). follows:

The inventory of building space, including funded construction, is as

- Adequate: 50,600 gsm (544,500 gsf) that require maintenance such as painting, repairs, and minor alterations;

- Substandard, can be made adequate: $82,500 \mathrm{gsm}(887,800 \mathrm{gsf}$ that do not meet existing standards-about $20 \%$ require minor rehabilitation (in electrical, structural, and mechanical systems), and the balance require major rehabilitation (for existing or projected program requirements); and

- Substandard, cannot be made adequate: $20,100 \mathrm{gsm}(216,700 \mathrm{gsf})$ that cannot be upgraded or rehabilitated at a cost less than new construction. 
Condition of Laboratory space fexcluding proposed FY 199.3 construction).

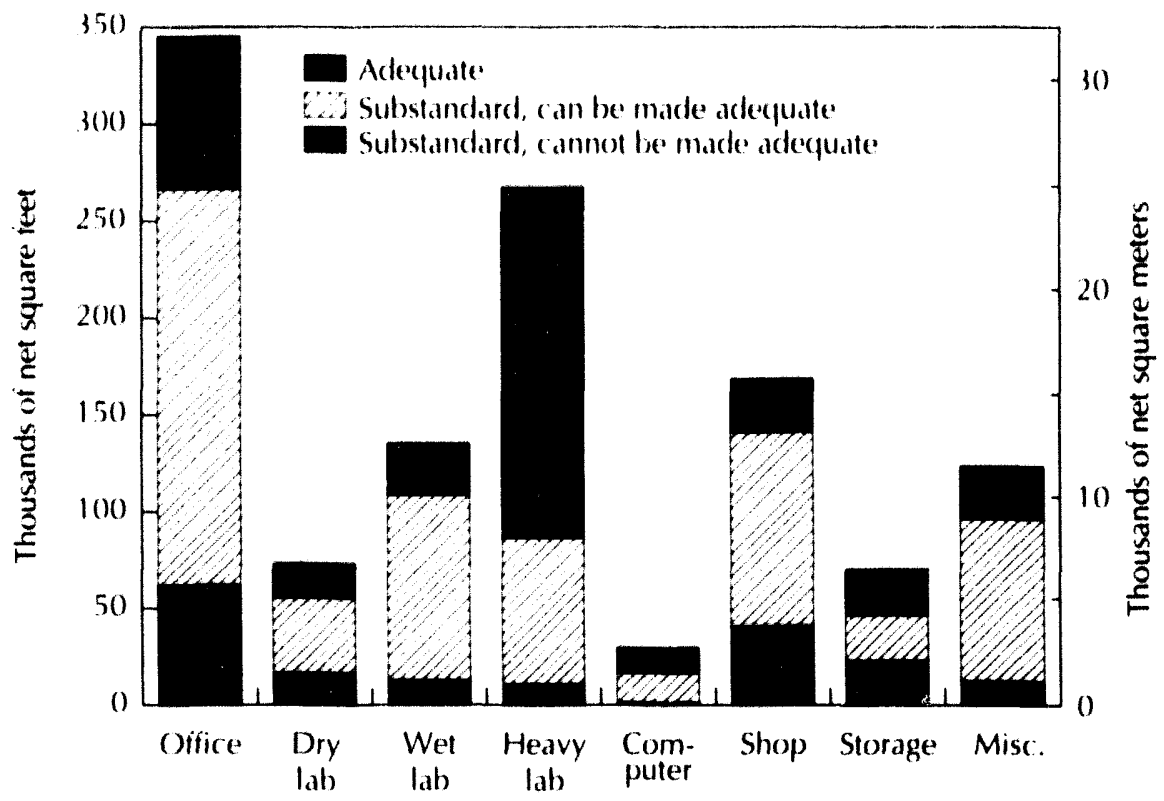

Facilities Replacement Value

\begin{tabular}{lcc}
\hline Type & Value $(\$ M)$ & $\%$ of Total \\
\hline Buildings & 272.5 & 64 \\
Utilities & 123.9 & 29 \\
Miscellaneous & 28.8 & 7 \\
Total & 425.2 & 100 \\
\hline
\end{tabular}

"Estimated in 1986.

\section{FACILITIES PLANS AND OPTIONS}

The SDP is based on five LBL site master plan concepts. These concepts accommodate the facilities improvement needs within existing geophysical, environmental, and operational conditions. They provide a basis for understanding and evaluating the more detailed elements of specific projects, planned locations, and other site improvement projections. The site planning concepts are to:

- Consolidate activities within seven functional planning areas to enhance efficiency and effectiveness and to provide specialized research facilities;

- Redevelop obsolete buildings and infrastructure, eliminate temporary structures used for permanent functions, and improve building arrangements to increase safety and energy efficiency;

- Concentrate development along the east-west circulation and utilities axis to enhance transportation and service systems, e.g., develop offroad parking and improve pedestrian pathways; 


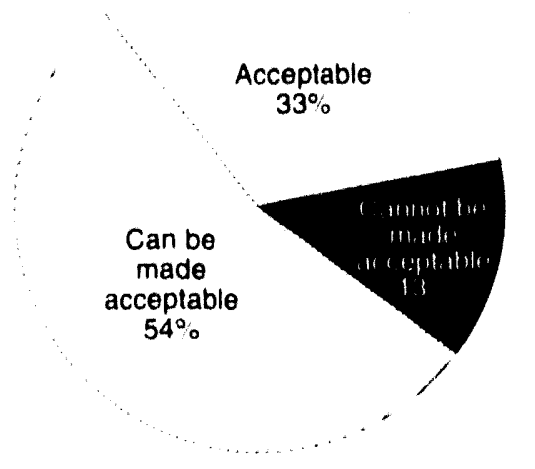

Condition of Laboratory space (excluding proposed fy 199.3 construction).
- Improve and maintain perimeter and internal buffer zones to screen noise-generating activities and minimize potential incompatibility between adjacent operations; and

- Provide off-site facilities for receiving, warehousing, and other support and research activities suitable for decentralization.

Facility planning includes exploring such options as the potential interim uses of off-site facilities for administrative and other support functions. Over the past three decades administrative requirements have been increasing as safety, accounting, environmental, and other requirements are addressed. LBL is exploring long-term options, such as reconfiguration of some existing space, new additions of priority office and support buildings, and the removal of temporary office structures. These temporary office structures proliferated during the past several decades as a result of limited construction for offices and support buildings.

If the full programmatic capability of the site is developed to meet anticipated national needs in energy technology and supporting research, the sites and new buildings would result in a net increase of $37,000 \mathrm{gsm}(0.4 \mathrm{Mgsf}$ to the existing main site, for a total of approximately $186,000 \mathrm{gsm}(2.0 \mathrm{Mgs}$ ). For comparison, the 1992 total, including current construction, consists of 153,000 $\operatorname{gsm}(1.65 \mathrm{Mgs}$ ) at the main site. The Laboratory's on-site space is now $100 \%$ utilized at an approximate $70 \%$ net to gross area efficiency. The useable onsite space is approximately $102,000 \mathrm{gsm}(1.1 \mathrm{M}$ net square feet). The building utilization efficiency is not projected to change significantly, although the efficiency of land use is expecied to improve with the replacement of obsolete single- and two-story buildings with three- to five-story structures.

\section{GENERAL PURPOSE FACILITIES PLANS}

\section{Multiprogram Energy Laboratory Facilities Support}

The total proposed and budgeted five-year MEL-FS program represents an investment need of $\$ 190.1 \mathrm{M}$. This modernization program addresses needs primarily related to the many buildings and utilities that are 20 to 40 years old. Individual projects are evaluated against generic Laboratory site development priorities such as safety, environmental protection, reliability, maintainability, design standards and obsolescence, and delivery of research and support services. Full modernization of the site would require a longterm 20-year investment program.

Proposed MEL-FS Construction Projects FY 1994-1998

\begin{tabular}{lc}
\hline Category & TEC (\$M) \\
\hline Safety, health, and environment & 64.0 \\
Mechanical utilities $^{\mathrm{a}}$ & 16.4 \\
Electrical utilities $^{\mathrm{a}}$ & 17.4 \\
Building rehabilitation/additions $^{\text {Total190.1 }}$ & 92.3 \\
\hline
\end{tabular}

"Also includes health- and safety-related building and utility improvements. 


\section{Safety and Health Improvements}

Health and safety improvements are needed in safety services, health services, building illumination, radiation protection and monitoring, and in other safety systems (see Section 6). Many of these projects were initiated as MEL-FS projects. Examples of important funded projects include Slope and Seismic Stabilization (required in the Bevalac and Shops areas), the Original Labsite Substation Project, the Instrument Support Laboratory Rehabilitation, the Building 90 Seismic Rehabilitation, a Fire and Safety Systems Upgrade Project (Phase I), and Hazardous Materials Saleguards Program (Phase I).

Road safety improvements are required for safe and efficient operations and movement of staff and materials throughout the site. New facilities, the Safety and Support Services Facility and an Environmental Monitoring and Industrial Hygiene Building, are necessary to correct deficiencies identified by the 1991 Tiger Team Assessment (see Section 6). These facilities will allow the Laboratory to meet accepted standards for LBL's environment, health, and safety programs, including providing adequate monitoring and sample processing laboratories, adequate emergency command and response facilities, and sufficient space for on-site offices for industrial hygiene, environmental, and other essential ES\&H staff.

In addition, other safety-related projects are needed for asbestos removal from the Health Services facility, fire safety, and emergency egress, as examples. Also, roads need widening, base materials need to be replaced to conform to current standards, acute curves and blind spots need to be eliminated, and road beds and adjacent slopes on steep hillsides need to be stabilized. As an example of long-range safety needs, the plan calls for three phases of road rehabilitation as MEL.FS projects.

\section{Mechanical Utilities/Safety}

The Laboratory's mechanical/utility systems are up to 40 years old. Mechanical utilities consist of domestic- and cooling-water, storm-drain and waste, natural-gas, compressed-air, and vacuum systems. Recent MEL-FS funding for Phase $I$ of the Mechanical Utilities Rehabilitation Plan has improved several critical mechanical systems, but nearly $60 \%$ of existing equipment is more than 20 years old and beyond its useful service life. Full implementation of LBL's Six-Phase Plan would minimize the possibility of accidents or program disruption by loss of essential utilities and equipment.

\section{Electrical Utilities/Safety}

LBL's power-distribution system consists of 24 substations and $32 \mathrm{~km}$ (20 miles) of $12-\mathrm{kV}$ primary distribution cable. Much of the distribution equipment and cables are beyond their expected service lives, resulting in reduced reliability and increased maintenance. The electrical rehabilitation projects have been prioritized into a six-phase program based on the expected failure rates of equipment and importance to site-wide facilities demands. Phase one, Cirizzly Peak Substation, has been completed. Phase two, Original Labsite Substation, and phase three, East Canyon Electrical Safety Project, were funded in FY 1992. The three remaining phases, Blackberry Switching Station Replacement, Central Switching Station and Feeders, and the Upper Blackberry Switching Station, are proposed projects included in the Twenty-Year MEL-FS Plan. The upgraded switching and distribution system will provide the reliability, flexibility, and expandability necessary for efficient Laboratory operation and future growth. 


\section{Building Replacement and Modernization}

The MEL.FS building replacement and modernization plan responds to the needs for salety, support and research infrastructure, and for general. purpose engineering facilities. Improvements in the condition of substandard space, as part of a long-erm modernization program, would enhance conditions for safely and efficiently achieving the Laboratory's mission. Included in the long-range plan is the removal of substandard facilities that cannot be made adequate. Projects include upgrades and ardditions for facilities, engineering and maintenance services, administrative services, applied sciences, science education and visitor facilities, and for integrated communic ations and computing.

\section{General Plant Projects}

Funding to date has been inadequate to meet the Laboratory needs within a timely schedule. This program has a significant backlog of projects, exceeding \$30 M. Roughly a third of this backlog is for environment, health, and safety needs; a third is for utilities safety and reliability, building maintenance, and standards compliance; and a third for multiprogram support facilities and small programmatic projects and additions. In FY 1993 more than half of the $\$ 3.3 \mathrm{M} \mathrm{CPP}$ budget will be used for environment, safety and health-related projects. The remaining funds cannot meet current project needs and do not allow progress in reducing this backlog. Increasing Cipp funding to $\$ 6 \mathrm{M}$ annually and increasing the ceiling from $\$ 1.2 \mathrm{M}$ to $\$ 2.0 \mathrm{M}$ would ensure the success of the Laboratory's safely rehabilitation program and help reduce the current backlog of projects over the next five years.

\section{General-Purpose Equipment}

In the past, essential support equipment has been funded through DOE's Nuclear Physics Division. LBL's Five-Year CPE Plan identifies needs based on DOE prioritization criteria, including those coupled to environment, safety, and health; legal renuirements; failed, worn, inefficient, or obsolete equipment; substandard performance; or increased workload and demand. The current funding level of $\$ 1.7 \mathrm{M}$ year is inadequate to meet the Laboratory needs. As noted in the new DOE/UC contract, "a continuing, active and affirmative program of supplementing and replacing such equipment is essential to scientific progress." Currently there is a $\$ 38 \mathrm{M}$ equipment backlog, which includes equipment for environmental monitoring, transportation, data processing, and communications. Increasing GPE support to $\$ 3 \mathrm{M} /$ year would provide a basis for reducing the backlog.

\section{Maintenance Plans}

Maintenance plans and budgets are developed annually within an overall five-year planning and safety management strategy. The Laboratory has improved its current maintenance scheduling system and backlog of maintenance projects through implementation of the site-wide Plant Inspection and Maintenance System Upgrade Program and is an active participant in the development of the Condition Assessment Inspection Service. These include noncapital alterations, general plant projects, and multiprogram general-purpose line items. Requirements are identified by periodic reviews and inspections, and new priorities are developed during the fiscal year. 


\section{LAWRENCE BERKELEY LABORATORY CONSTRUCTION PLAN \\ FY 1994 - FY 1999}

Pian for Programmatic and Coneral Purpose Facillitee, Including Funded, Budgoted and Proposed Conatructlon (FY BA, sM)

\begin{tabular}{|c|c|c|c|c|c|c|c|c|c|c|}
\hline Project & TEC & 1994 & 1995 & 1996 & 1997 & 1998 & 1999 & 2000 & $200 !$ & 2002 \\
\hline 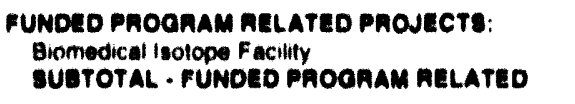 & $\begin{array}{l}23 \\
2.3\end{array}$ & & & & & & & & & \\
\hline 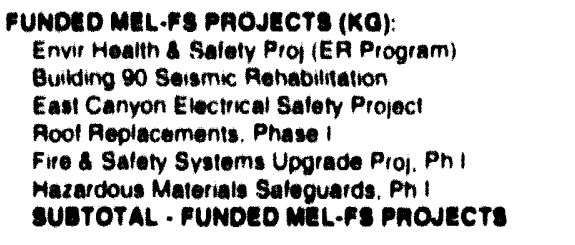 & $\begin{array}{r}13163 \\
6800 \\
3900 \\
2500 \\
4600 \\
5100 \\
30.003\end{array}$ & $\begin{array}{l}1691 \\
1,608 \\
1000 \\
1000 \\
6.200\end{array}$ & $\begin{array}{l}1000 \\
2000 \\
2500 \\
8.600\end{array}$ & $\begin{array}{l}1100 \\
1100 \\
2.200\end{array}$ & & & & & & \\
\hline 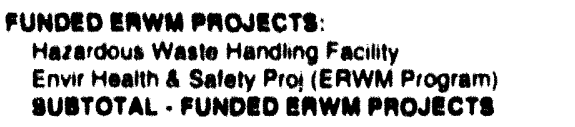 & $\begin{array}{r}120 \\
11 \\
13.1\end{array}$ & $\begin{array}{l}5.8 \\
0.0 \\
8.0\end{array}$ & & & & & & & & \\
\hline TOTAL FUNDED & 81.8 & 11.1 & 8.8 & 2.2 & & & & & & \\
\hline $\begin{array}{l}\text { BUDOETED PAOGRAM AELATED PAOJECTS } \\
\text { Human OEnome Laboralory (KP) } \\
\text { ALS Structural Biotogy Support Facilities (KC) }\end{array}$ & $\begin{array}{r}247 \\
79\end{array}$ & $\begin{array}{l}2.2 \\
0.6\end{array}$ & $\begin{array}{r}156 \\
47\end{array}$ & $\begin{array}{l}59 \\
26\end{array}$ & 10 & & & & & \\
\hline $\begin{array}{l}\text { DUDOETED MEL.FS PAOJECTS (KO) } \\
\text { No new pinfect slarts in FY } 1994\end{array}$ & 0000 & 0000 & 0000 & 0000 & & & & & & \\
\hline TOTAL FUNDED and BUDQETED & 4.1 & 13.0 & 25.8 & 10.7 & 1.0 & & & & & \\
\hline $\begin{array}{l}\text { PAOPOSEO PROOAAM AELATEO PAOJECTS*: } \\
\text { ALS Beamines Initialive (KC) } \\
\text { Induction Linac Systems Experiment (AT) } \\
\text { Chemical DYnamics Research Laboratory (KC) } \\
\text { sUbTOTAL. PAOPOSED PAOQAAM RELATEO }\end{array}$ & $\begin{array}{r}650 \\
610 \\
559 \\
298.6\end{array}$ & 16.7 & $\begin{aligned} 150 \\
8.3 \\
71 \\
76.8\end{aligned}$ & $\begin{array}{l}20.0 \\
131 \\
169 \\
09.2\end{array}$ & $\begin{array}{l}150 \\
14.0 \\
16.2 \\
47.2\end{array}$ & $\begin{array}{l}100 \\
135 \\
102 \\
33.7\end{array}$ & $\begin{array}{r}50 \\
121 \\
55 \\
22.6\end{array}$ & & & \\
\hline PAOPOSED MEL-FS PAOJECTS: & & & & & & & & & & \\
\hline Salety \& Suppon Services Facility & 123 & & 12 & 61 & 50 & & & & & \\
\hline $\begin{array}{l}\text { Uogrd of Site Mech Uil Ph II. Sewer Monit } \\
\text { Envit Monitoring \& Industial Hygiene Bidg } \\
\text { Roadway Safety \& Stabilization. Phase I } \\
\text { Sanitary Sewer Restoration }\end{array}$ & $\begin{array}{r}91 \\
249 \\
67 \\
26\end{array}$ & & & $\begin{array}{l}09 \\
50 \\
07 \\
03\end{array}$ & $\begin{array}{r}63 \\
119 \\
46 \\
17\end{array}$ & $\begin{array}{l}19 \\
80 \\
1.4 \\
06\end{array}$ & & & & \\
\hline $\begin{array}{l}\text { LBL Condult Intrastructure Improvements } \\
\text { Upgrade of LBL Communications System } \\
\text { Facilties Buiding } \\
\text { Medical Serv Asbestos Abatement \& Rehab }\end{array}$ & $\begin{array}{r}39 \\
47 \\
165 \\
31\end{array}$ & & & & $\begin{array}{l}04 \\
05 \\
16 \\
03\end{array}$ & $\begin{array}{r}2.7 \\
32 \\
117 \\
22\end{array}$ & $\begin{array}{l}08 \\
10 \\
32 \\
06\end{array}$ & & & \\
\hline $\begin{array}{l}\text { Mecranical Equipment Replacement. Ph I } \\
\text { Root Replacements. Ph II } \\
\text { Elec Sys Rehab. Ph IV. Bikby Swit Sta Replc } \\
\text { Olu Town Parking Structure }\end{array}$ & $\begin{array}{l}50 \\
75 \\
74 \\
35\end{array}$ & & & & & $\begin{array}{l}05 \\
07 \\
07 \\
03\end{array}$ & $\begin{array}{l}35 \\
54 \\
53 \\
26\end{array}$ & $\begin{array}{l}10 \\
14 \\
14 \\
06\end{array}$ & & \\
\hline $\begin{array}{l}\text { Blackberry Canyon Parking Structure } \\
\text { Admin Services Addn. Bidg } 50 E \text { F } 2 \text { nd Fl } \\
\text { Fire \& Satery Systems Upgrd Prolect Ph II } \\
\text { Hazardous Matertais Safeguards. Phase II }\end{array}$ & $\begin{array}{r}229 \\
94 \\
56 \\
81\end{array}$ & & & & & & $\begin{array}{l}23 \\
09 \\
06 \\
08\end{array}$ & $\begin{array}{r}160 \\
67 \\
38 \\
57\end{array}$ & $\begin{array}{l}46 \\
18 \\
12 \\
16\end{array}$ & \\
\hline SUETOTAL - PAOPOSED MEL-FS PAOJ'CTSS & 183.2 & 0.0 & 1.2 & 13.0 & 32.3 & 33.9 & 27.0 & 36.6 & 9.2 & 0.0 \\
\hline TOTAL FUNO, GUDOT \& PAOP MEL.FS 'JAOJ & 189.3 & 5.3 & 6.7 & 16.2 & 32.3 & 33.9 & 27.0 & 36.6 & 9.2 & 0.0 \\
\hline
\end{tabular}

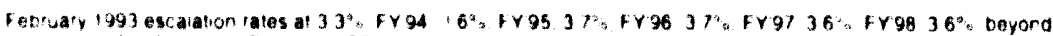

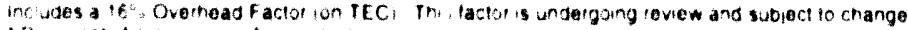

Prosestrom previous tisca years

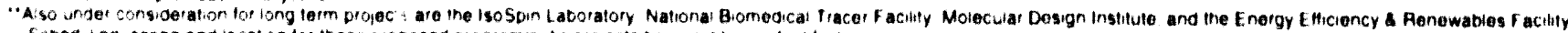

scheduling sope and bcation tor these proposes programmatic propects have not been ifentitiod 


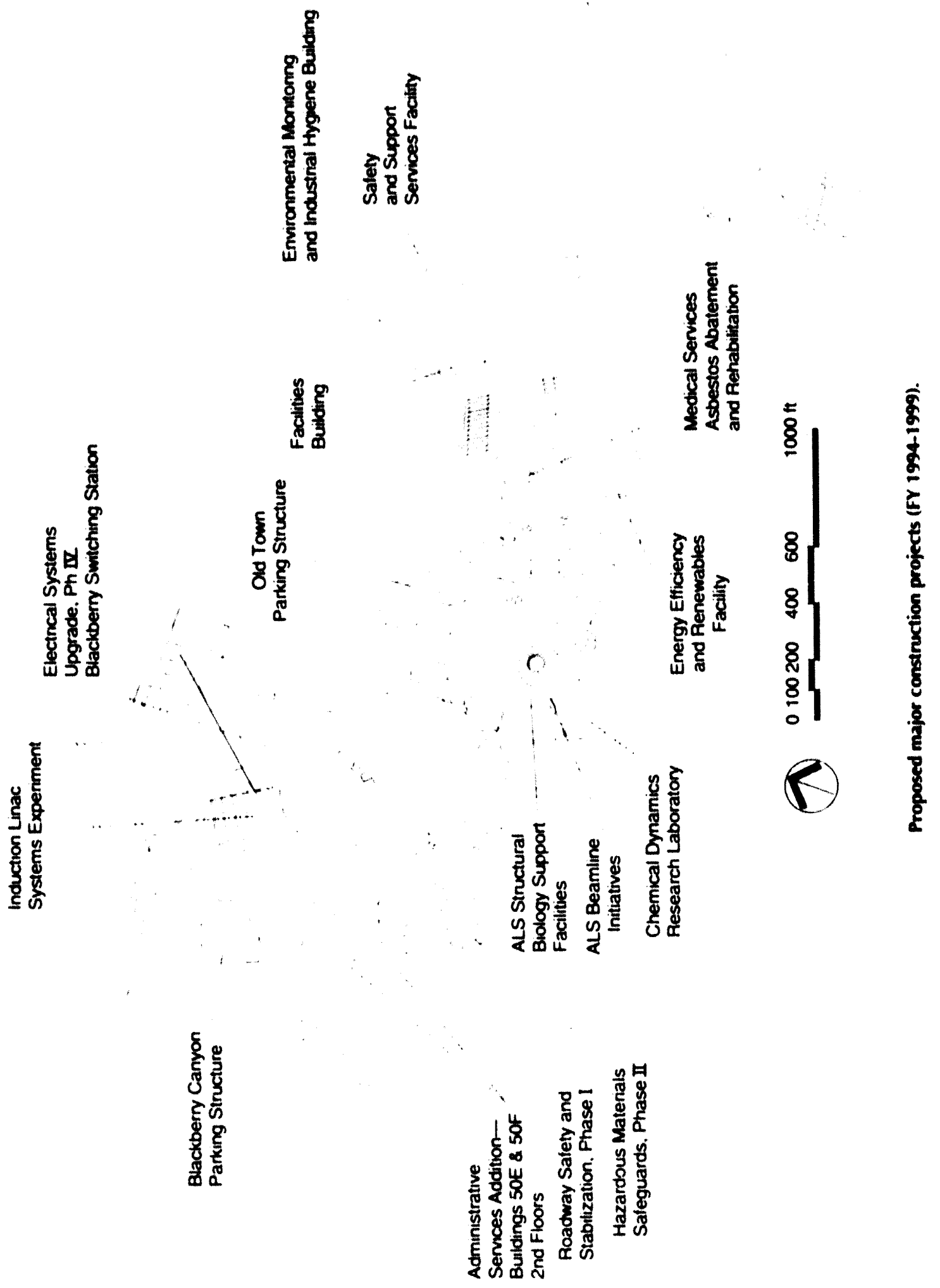


As discussed in the MEL. FS and ERWM sections abeve, long-range site modernization also addresses many of the major maintenance issues. The road safety projects, slope and seismic stabilization, general-purpose-facilities replacement projects, and other environmental and safety improvements are examples. The operating expenses for maintenance include physic al-plant maintenance, mobile-equipment maintenance, and specialized maintenance related to shop, computer, and telecommunications facilities.

The current strategy for improving maintenance relies on strengthening the capital outlays, continuing the operating-costs efforts, and implementing the maintenance planning system as indic ated above. This allows the Laboratory to sustain DOE facilities while planning for maintenance cost economies. These economies can be achieved through the replacement of existing obsolete and high-maintenance-cost facilities with modern facilities and equipment supported by increased MEL.FS, CPP, and CPE funds.

\section{PROGRAMMATIC FACILITIES PLANS}

The new programmatic research buildings and facilities in the plan serve the national interest in several research areas where L.BL. has established programs. Several major scientific facilities form the core of LBL's plans to contribute to DOE's research capabilities (see pages 9.8 and 9.9 for table and accompanying figure). The Human (ienome Laboratory, ALS Structural Biology Support Facilities, Chemical Dynamics Research Laboratory, Induction Linac Systems Experiments, and ALS Beamline Initiative are significant resources for programs supported by the DOE's Office of Fusion Energy, Chemical Sciences Division, and Office of Health and Environmental Research. Completion of the second floor of the ALS building for users is essential to meet ALS program requirements. The Laboratory is also in the initial stages of planning for an IsoSpin Laboratory, National Biomedical Tracer Facility, Energy Efficiency and Renewables Facility, and a Molecular Design Institute.

\section{FACILITIES DECOMMISSIONING PLAN}

The development of new national program directions for nuclear physics has resulted in the shutdown of LBL's Bevalac nuclear physics program during FY 1993. The Bevalac decommissioning plans are being developed between I.BL, O):R, EM-60) and EM-40. Operations were curtailed in mid-FY 1993. A stand-down and secure phase will be conducted by L.BL in the remainder of $F Y$ 1993 and into FY 1994. Subsequent planning, activities and funding are being identified by the responsible DOE office and L.BL.

The Laboratory conducts periodic reviews of facilities that may become inactive. Other facilities to be decommissioned inc lude garmma irradiators. 
Facilities Decommissioning Plan (\$M)

\begin{tabular}{lccccccc}
\hline Project Phase & FY 1994 & FY 1995 & FY 1996 & FY 1997 & FY 1998 & FY 1999 \\
\hline Bevalac: & & & & & & \\
$\quad$ Stand-down and Secure (OER) & 4.0 & 2.0 & - & - & - & - \\
Transition (EM-60) & 1.15 & $3.0^{4}$ & 3.0 & 3.0 & - & - \\
D\&D (EM-40)" & - & - & - & - & 15.0 & 21.0 \\
Gamma Irradiators (OER) & 0.3 & - & - & - & - & - \\
D\&D Base Program (OER) & 0.4 & 0.6 & 0.6 & - & - & - \\
$\quad$ Total & 5.85 & 5.6 & 3.6 & 3.0 & 15.0 & 21.0 \\
\hline
\end{tabular}

IFTPA for \$3 M in FY 1995 submitted to ()ER pending commitment of EM-60) funding.

"Tolal D\&D estimate for the Bevalac fo completion in FY $2002 \$ 75 \mathrm{M}$.

\section{FACILITIES RESOURCES REQUIREMENTS}

A five-year construction plan for programmatic and general purpose facilities is provided in the L.BL Five-Year Construction Plan table on page 9.8. MEL-FS proposed projects are listed in a prioritized order of sequence in accordance with CAMP criteria. All budget information as indicated is actual-year authority. 


\title{
10 INFORMATION RESOURCES MANAGEMENT
}

\author{
Our operational, administrative, and technical resources \\ will integrate secmlessly with the research and engineering \\ programs to make an LBL that works.
}

LBL Vision 2000

LBL's information resources program is a pioneer in the development of network-based, "location-transparent," distributed computing. This program supports the national High Performance Computing and Communications Initiative, the next generation of U.S. "information highways," and the proposed National Information Infrastructure. Through the Laboratory's Information Resources Management (IRM) planning function, these national efforts are coordinated with LBL's own mission.

This section identifies IRM planning goals, summarizes the present environment and IRM organization, and describes the strategy and initiatives underlying the management of our information resources. Information resources directly complements and enables LBL's human resources-its scientific, technical, and support staff. It also complements the need to plan for support of the staff in conjunction with improved site and facilities resources.

\section{GOALS AND OBJECTIVES}

\section{National Context}

LBL's information resources directly support DOE national goals that are an important element of the Laboratory's IRM program. The research programs conducted at LBL make essential use of information technology for data collection and analysis, modeling of physical processes, project management, administration, and the preparation and publication of technical reports. LBL continues to support DOE strategic goals through: 
- Providing a computational and analytical basis to DOE programs for securing future energy supplies. For example, LBL is developing models and technologies to image structures and processes in the earth's crust, including electromagnetic methods for high-resolution mapping, methods for signal processing, and measuring anisotropy in geological formations.

- Contributing to computing industry collaborations that assist DOE programs for economic competitiveness in critical technologies. LBL collaborates with industry on hardware and software, including the application of network technology to computer-assisted cooperative work, network performance, and internet working protocols.

- Developing databases that underlay DOE programs for protecting health and the environment. LBL leads in the development of the Comprehensive Epidemiological Data Resource, providing nationwide epidemiological information. Populations At Risk to Environmental Pollution (PAREP) develops biostatistical and computational techniques for geographic analysis of disease risk factors.

LBL conducts programs for the Office of Energy Research and other partners as described in Section 5, Scientific and Technical Programs. Central to these programs are computational research and networking systems for Basic Energy Sciences, Health and Environmental Research, and High Energy and Nuclear Physics through:

- Advancing models and databases that underpin Basic Energy Sciences programs to develop new insights in chemistry, materials sciences, geology, and computing science. For example, the Scientific Database Management Research Program investigates the structural differences between scientific data and conventional data and develops storage, organization, and new data-modeling methods.

- Assisting DOE information management for fundamental health and environmental research. As an example, the Laboratory Information Management System (LIMS) is a computer-assisted molecular biology laboratory notebook that supports sequencing protocols.

- Developing high-speed hardware, software, and process simulations for high energy and nuclear physics. Sophisticated detectors for investigating the basic constituents of matter at high energy and luminosity are designed by LBL to meet the new megahertz data-rate demands with new systems for readout and analysis for the DO and the CDF detectors at Fermilab, the Solenoidal Detector at the SSCL, and STAR at RHIC (Sections 4 and 5).

LBL also develops information technology in collaboration with other laboratories, universities, and industry that supports DOE's mission for technology transfer. For example, LBL develops improved systems for modeling energy use and loss in buildings, which receive widespread application and distribution by the energy conservation industry. In the computer hardware area, LBL completed CRADAs for the joint development of disk-drive hardware with two major manufacturers in FY 1992. In supporting DOE's science and engineering education mission, $L B L$ provides access to its information resources to nearly 500 hundred graduate students and several hundred undergraduates. Part of the education these students receive is an introduction to the application of sophisticated computational and visualization tools and techniques to research problems. 


\section{Strategic Goals}

The Laboratory has developed IRM goals to support research and development, corporate information management, technical information requirements, and records management. The IRM goals are to:

- Conduct an advanced research and development program in support of the nation's High Performance Computing and Communications Initiative and the most effective development and utilization of national information highways.

- Provide state-of-the-art and transparent computing and communications resources for DOE programs and service to every scientific, engineering, or administrative employee, including advanced network communications technology that keeps pace with demand; workstation support services and technical support for telecommuting and telework; transparent access to computing resources; upgraded central computing facilities; and proper control of access to sensitive files.

- Define and implement a corporate information management architecture that includes interoperable electronic mail, makes corporate databases fully accessible, implements file portability, and strengthens institutional standards for database systems and utilities.

- Supply effective scientific and technical information services for those functions that the Laboratory can provide more efficiently and timely than through commercial services, and introduce appropriate new digital technology within funding constraints.

- Maintain a secure records management program that identifies and protects the essential scientific and administrative records of the Laboratory and administers appropriate retention schedules for all LBL records.

These goals underpin LBL's mission for research and development, design and operation of user facilities, education and training, and technology transfer. Together with the human and facilities resources of the Laboratory, the information resources enable a flexible and responsive operating environment for the implementation of DOE programs. Effective information management is vital to the success of this mission, and will require the allocation of adequate DOE resources for effective implementation.

\section{CURRENT SITUATION}

\section{Technology Resources}

LBL's technology for information resources management includes access to a broad spectrum of computing platforms, storage devices and network facilities, a range of software for research, administrative, support functions, and integrated telecommunications and special purpose communications systems. A key element of LBL's long-range strategy is a program of focused replacement and improvement of these resources to support DOE's programs and institutional support requirements.

LBL's on-site computing resources include powerful computing processor clusters, storage, and networking facilities in the central scientific center (based on the VMS and UNIX standards), smaller business computing 
facilities in the administrative data processing center (based on IBM and UNIX standards), and a range of divisional servers and systems. All professional and administrative staff are provided with desktop systems. Specialized servers in the central scientific center include a mass storage system and a scientific visualization system. Access to OER supercomputer centers at Lawrence Livermore National Laboratory and Florida State University is also provided. A new system for parallel computing will be installed in the third quarter of FY 1993.

Software for individual use on single-user or workgroup computers is obtained commercially in most cases; some small applications are developed for specific, limited use. Software that supports unique experimental devices is developed within rigorous formal management appropriate to the experiment in question. Software for general applications-scientific and administrative-is developed using conventional software development techniques, including appropriate reviews. As projects are completed, those not LBL-specific are submitted to DOE's Energy Scientific and Technical Software Center.

LBL operates an on-site Integrated Communications System (ICS) for voice and data switching with a 7500 line capacity and 390 trunks. The system is being acquired on lease-to-ownership, with ownership to take place in FY 1997. LBL operates a Laboratory-wide Local Area Network (LBLnet) that provides internal and external network services for all computer, workstation, and personal computer systems, connecting 81 buildings and 70 trailers on the LBL site. LBLnet also provides connections to external networks (e.g., ESnet, BARRNET, and the Internet). LBL is a leading participant in the Bay Area Gigabit Testbed to advance regional and national communication infrastructure. Special purpose communications systems, including the Fire Alarm System, the Cardkey Access Control System, and the Energy Management Control System, use both ICS circuits and dedicated lines installed throughout the site for signal transmission. In addition cellular telephones are utilized where appropriate to provide voice contact with personnel in the field and are available to serve as backup to the regular telephone system and radios in emergency situations.

Laboratory radiocommunications systems consist of 17 base stations, 64 mobile stations (including transmitters in rescue boxes distributed about the LBL site), and approximately 195 portable stations. These provide two-way radio communications for service and emergency functions, such as transportation of materials, fire, police, and safety services, and also support voice and data telemetry for research field crews. LBL also supports microwave links, including those to LLNL and SLAC. LBL has been a leader in the ER Videoconference Network (ERVN), a facility that includes several US and foreign laboratories. ERVN is being expanded to interconnect with DOE headquarters and field offices at San Francisco and Chicago. Also planned is the evolution away from shared ESnet bandwidth resources toward service with greater connectivity based on Video Telecom codec equipment.

Existing information infrastructure must be upgraded and replaced in a timely manner. New demands for extremely high data rates, reliance of shared resources, and the need for improved communications call for new computational power, higher-speed networking resources, and more advanced technical information systems for document preparation, transmission, and storage. The Laboratory anticipates that these institutional and distributed resources will be provided in the most cost-effective manner through equipment and capitalized projects supported by the Office of Energy Research. 
Information Resource Management Resource Requirements (FY BA, \$M)

\begin{tabular}{lrrrrr}
\hline Category & 1992 & 1993 & 1994 & 1995 & 1996 \\
\hline Recharge & 8.7 & 9.6 & 10.1 & 10.6 & 11.1 \\
Overhead & 5.0 & 4.3 & 3.6 & 3.4 & 3.7 \\
\hline
\end{tabular}

No program funding has been received.

\section{IRM Organization and Management Resources}

DOE and LBL are strengthening management of information resources to improve the effectiveness, efficiency, reliability, and security of information technology. Through DOE and in partnership with industry $L B L$ is participating in national efforts to improve "information highways" to advance the nation's scientific and technical infrastructure. At $L B L$ the planning and operational management of information technology is focused in the Information and Computing Sciences Division.

- The Communications and Networking Resources Department has responsibility for developing the LBL's communications resources, including local and external connectivity. The department reviews divisional plans to ensure consistency with LBL policies and plans, and manages telephone services and the LBLnet.

- The Office of Information Technology Resources Planning has responsibility for developing policy and coordinating planning for LBL computing technology, including equipment acquisitions and computer security. The office coordinates long-range plans with institutional and site development plans in the Office for Planning and Development.

- The Computing Resources Department maintains and operates the Central Scientific Computing Facility, provides system maintenance support for small distributed systems, maintains access to the National Energy Research Supercomputing Center, and provides a broad range of workstation support services.

- The Technical Information Department has responsibility for centralized printing and publishing services, including technical writing and editing, illustration and photography, operation of the LBL Printing Plant, conference coordination, and video and audio services.

- The Systems Engineering Department, in the Engineering Division, designs and builds computer control and data acquisition systems for LBL programs, conducts research in networks and provides research and engineering software, and supplies hardware maintenance for workstations.

- The Information Systems and Services Department, in the Administration Division, provides administrative computing services, including payroll, stores, and accounting functions. It also provides interactive access to centralized databases for business reports. The LBL archivist, also in the in the Administration Division, has responsibility for archives and records management.

Smaller computing functions exist within many of the Laboratory's research organizations. They are dedicated to computer applications support in a particular research discipline. 


\section{Recent IRM Accomplishments}

The Laboratory is currently participating in a range of DOE/ER-wide and project-specific efforts to enhance the development of information technology and management. Examples of these efforts in FY 1992-93 serve to highlight LBL's current information management activities and computing environment:

- New networking technologies for distributed computing resulted in improved protocols, including completely distributed applications. $L B L$ is also developing high-performance protocols that make effective use of network bandwidth at high (megabit-to-gigabits/second) and low ends (300-56,000 bps).

- Installing an artificial intelligence-based administrative software application for handling job applications and résumés. Pilot use will begin in early calendar year 1993.

- Undertaking a phased implementation of Fiber Distributed Digital Interchange, including beta-testing of commercial implementations, over the next several years.

- Selecting a new system for parallel computing, scheduled for installation in the third quarter of FY 1993.

- Designing new methods for networking and handling the real-time video conferencing on existing computer networks such as ESnet. LBL is an active participant in the BLANCA, Bay Area Gigabit, and MACIC gigabit networking testbeds. LBL is participating in the ESnet T3 Upgrade project through testing technology concepts and demonstrating high-performance applications at speeds beyond $\mathrm{T} 1$.

\section{STRATEGIES}

LBL's strategy for IRM includes focused support for improving the nation's computing and communications infrastructure, assistance to DOE in the development of IRM policies and plans, and the development of LBL's computing and communications infrastructure to support the LBL DOE multiprogram energy research Laboratory mission. LBL's strategies directly support IRM goals and objectives described above to provide state-of-theart, transparent computing and communications resources, accessible corporate information management systems, effective scientific and technical information services, and a secure records management program.

LBL proposes a strategy for internal telecommunications resource development funding that supports institutional programs for DOE rather than fragmented project by project costs that are difficult to determine. LBL telecommunications costs are accrued by the LBL organization, not by project. For example, telephone costs are billed to the telephone used to make the call, and hence to the division responsible for the charges accumulated from that instrument. Meaningful assignment of these costs to specific programs is not possible. On this basis, increased GPP and GPE for information infrastructure is critical. 


\section{External Strategies}

\section{Improve National Computing Infrastructure}

To develop DOE's Information Resources Management strategies, LBL. has provided continuing support to the Office of IRM Policy. This includes review of existing strategies and policies and the development of new policies to cope with the changing IRM environment. LBL is active in the IRM Planning Process Improvement Team through direct participation in the Team itself and through participation in a dual pilot program instituted by OER to test various methods for increasing participation by program managers in the planning process. LBL strongly supports DOE's efforts to develop a planning process that is driven by programmatic need rather than by reporting requirements. LBL is interested in advancing both DOE's process improvement team and in the OER pilot projects that supplement it.

LBL is active in several branches of the standards-development process that are of particular interest to DOE. These include participation in technical committees dealing with network issues and text and office applications and languages, the planning and development of ESnet, the Internet Engineering Task Force, and the planning and implementation of COSIP (Government Open Systems Interconnection Profile).

LBL also supports the development of the national communications infrastructure by providing the chairperson of the ESnet Steering Committee, which also assists in the prioritizing at DOE developments in high-speed networking and video conferencing.

\section{Implement Distributed Computing and Telecommuting}

To advance $L B L$ strategic planning to improve distributed computing and the working environment, LBL is initiating in FY 1993 a project to investigate the requirements and consequences of telecommuting in the DOE environment. LBL is also developing prototypical software to define and refine mechanisms that facilitate the use of distributed, disparate computers linked by high-speed networks to work cooperatively on single problems. In essence, using temporary connections over the network, the user creates a virtual computer with a number of special-purpose processors, each of which is optimal for some portion of the task the user is trying to complete. Such virtual computers are expected to have many applications in both research and education.

\section{Enhance International Collaborations and Information Environment}

In support of DOE and Laboratory goals to increase the benefits of international cooperation in basic science, LBL engages in many activities that involve foreign collaborations. Among those that are IRM-intensive are those involving LBL's expertise in the numerical modeling of geophysical and geochemical processes. For example, there are joint projects on nuclear or chemical waste with Russia, Sweden, Switzerland, and Canada, and formal agreements with both Mexico and Italy on geothermal development. $\mathrm{LBL}$ is the site of the International Geothermal Association. 


\section{Internal Strategies}

\section{Enhance the LBL Work Environment and Corporate Information}

A new institutional strategic change to achieve the LBL Vision 2000 has been an increase in activity directed toward the definition and management of corporate and institutional data and information. LBL's site strategy encourages the integration of appropriate information technology into the individual work environments. The process of creating an integrated Laboratory approach to information management has been initiated. This effort will extend over several years. Activities in FY 1992 included an initiation of a database forum to exchange experience and to recommend Labwide policy; development of distributed databases for the reporting and analysis of self-assessment information; and initiation of projects to develop electronic time reporting and automated account authorization. LBL issued a Request for Information in FY 1993 addressing institutional needs for distributed database management. After analysis of the responses, one or more institutional standards will be adopted for management systems and specific applications. This is a first major step in a long-range effort to provide more effective management of institutional data. A second step is the planned replacement, also in FY 1993, of the 20 year old purchasing system.

Many of LBL's computing and communications facilities and planning requirements are institutional, and we believe that DOE should provide more vigorous support for institutional technology resources at the multiprogram energy laboratories. This strategy strongly supports the goals of DOE's Vision 21 because it reinforces the notion of institutionalization of IRM Planning, which is obscured by a reporting process that requires the partitioning of institutional requirements into a welter of small, fictitious, "programmatic" tabulations.

\section{Provide Quality and Timely Information and Records}

In support of LBL Vision 2000 and DOE's renewed emphasis on quality, LBL is placing increased emphasis on quality of operations consistent with DOE Orders 5480.19 (Conduct of Operations) and 5700.6B (Quality Assurance). These orders require the Laboratory to ensure that suppliers to LBL meet acceptable quality standards. LBL is seeking to implement this for all information resources and related services, including telecommunications and printing services. LBL encounters problems with GPO-approved nationally solicited printers who fail to meet quality standards in the initial press run. This leads to publication delays, often compounded by non-local travel to conduct press inspections. LBL will continue to work with DOE to resolve the problem to enable the timely preparation of quality technical documents.

\section{RESOURCES AND INITIATIVES}

LBL IRM initiatives span a range of significant new programs that will expand DOE computing and communications resources. The National High Performance Computing and Communications Initiative is described in 
Section 4. The following initiatives are directed at improving DOE and Laboratory infrastructure and management and are directed toward meeting the resource and management needs of the DOE and its Office of Energy Research.

\section{Advanced High-Speed Networking}

To meet projected traffic and demand, I.BL anticipates that expanded communications and networking infrastructure will be required during the middle of this decade. The Laboratory's High Speed Networking Initiative seeks to extend high-speed ( $100 \mathrm{Mbs}$ and beyond) network access to the majority of LBL workstations in order to relieve congestion and meet traffic demands. In addition, network backbones and server systems will need to be upgraded or replaced.

LBL also supports the implementation of the Government Open Systems Interconnection Profile (COSIP), a networking and interconnection protocol system that improves communications among government agencies and supporting contractors. LBL's GOSIP Transition Plan includes a period of coexistence between GOSIP and the protocols currently in use to ensure minimal disruption to ongoing programs and cost effectiveness. Initial elements of the LBL project include development of GOSIP services on LBLnet, participation in field tests and GOSIP Internet Gateway efforts, and development of compatible directory services on LBLnet.

Advanced High-Speed Networking Resource Requirements (\$M) ${ }^{\mathrm{a}}$

\begin{tabular}{lccccccc}
\hline Category & 1994 & 1995 & 1996 & 1997 & 1998 & 1999 & Total \\
\hline Operating & 0.4 & 0.8 & 0.7 & 0.3 & 0.2 & 0.2 & $2.6^{\mathrm{b}}$ \\
Construction & 0.0 & 0.0 & 0.0 & 0.0 & 0.0 & 0.0 & 0.0 \\
\hline
\end{tabular}

aPreliminary estimate of LBL actual year Budget Authority (B\&R code KC07, tentative). bIncludes $\$ 1.6 \mathrm{M}$ in equipment costs.

\section{Computing Upgrade}

LBL expects that new styles of computation, relying more heavily on parallel algorithms and intensive imaging and visualization services, will come into increasing use during the middle years of this decade. The existing cluster is poorly suited to accommodate such demands. The Laboratory's Computing Upgrade Initiative seeks to replace the existing computer cluster with more modern systems, expand our mass storage systems, and provide more generally accessible visualization services.

As a first step, in FY 1993 LBL will acquire a system for parallel computing to serve as a research platform for the development of parallel programming techniques and parallel algorithms. It will also serve as a source of improved performance on a class of problems involving complex operations on large arrays of data, such as occurrences in image processing, visualization, and image reconstruction. These problems arise in several areas of LBL research, including tomographic image construction, underground imaging, gene sequencing, and molecular docking. The next steps will involve significant new parallel and other computational and storage resources in both distributed and centralized locations. 
Computing Upgrade Resource Requirements (\$M)*

\begin{tabular}{lccccccc}
\hline Categoty & 1994 & 1995 & 1996 & 1997 & 1998 & 1999 & Total \\
\hline Operating & 0.4 & 0.4 & 0.8 & 0.5 & 0.5 & 1.0 & $3.6^{\mathrm{b}}$ \\
Construction & 0.0 & 0.0 & 0.0 & 0.0 & 0.0 & 0.0 & 0.0 \\
\hline
\end{tabular}

apreliminary estimate of LBL actual year Buctget Authority (B\&R code KC(17).

"Total is all equipment costs.

\section{Visualization, Video Conferencing, and Technical Information}

LBL is playing a lead role in organizing a testbed that will bring logether most of the major workstation vendors, several universities and laboratories, and the telecommunications industry to develop and test the hardware and software technology needed to support workstation-based video teleconferencing in a metropolitan area network. The project will also develop prototype technologies for tele-seminar.

LBL is also playing a lead role in the development of packet-based computing and communication environments to support collaborative work. This includes a multimedia environment incorporating audio, video, and a distributed electronic whiteboard operating over the InterneV/ESnet and a packet-based video-conferencing multicast environment that will greatly enhance ER-wide video conferencing.

LBL has begun a program of introducing modern technology into the operation of the Library and the Technical Information Departments. In past years, editors and illustrators have been provided with computer-assisted desktop workstations, and video technology has been upgraded. In FY 1992 LBL began the development of a digital darkroom, which will allow phasing out the use of wet-chemistry photography on site, and began installation of an on-line Library catalogue system, accessible to the whole LBL population.

LBL has begun implementation of the LBL node in a video conference facility being installed under the auspices of the UC Office of the President for purposes of facilitating contract management for the DOE laboratories. This system, based on Compression Labs CODEC equipment, is being built over dedicated commercial and UC systemwide data circuits, and will be operational in FY 1993.

Technical Information Resource Requirements (\$M)

\begin{tabular}{lccccccc}
\hline Category & 1994 & 1995 & 1996 & 1997 & 1998 & 1999 & Total \\
\hline Operating & 0.5 & 0.5 & 0.3 & 0.2 & 0.2 & 0.2 & $1.9^{\mathrm{b}}$ \\
Construction & 0.0 & 0.0 & 0.0 & 0.0 & 0.0 & 0.0 & 0.0 \\
\hline
\end{tabular}

"Preliminary estimate of LBL actual year Budget Authority (B\&R code KCO7).

"Includes \$( $) .4 \mathrm{M}$ in equipment costs. 


\section{Other Initiatives and Infrastructure Investments}

In FY 1993 LBL will undertake a program to assess the implications of telecommuting for scientific environments such as exist at DOE laboratories. As a separate initiative, and in common with other federal facilities, LBL has begun an examination of options to satisfy the impending narrowed bandwidth requirements for radio systems. Conversion to narrow bandwidth telecommınications using a trunk based system will require expenditures over the next several years, beginning in FY 1996. This program is to be coordinated among the DOE laboratories in response to the DOE requirements.

Other Initiatives and Infrastructure Investments

Resource Requirements (SM)*

\begin{tabular}{lcccccccc}
\hline Category & 1994 & 1995 & 1996 & 1997 & 1998 & 1999 & Total \\
\hline Operating & 0.3 & 0.3 & 0.5 & 1.0 & 1.5 & 1.5 & $5.1^{\mathrm{b}}$ \\
Construction & 0.0 & 0.0 & 0.4 & 0.0 & 0.0 & 0.0 & 0.4 \\
\hline
\end{tabular}

"Preliminary estimate of LBL actual year Budget Authority (B\&R code KC07).

bIncludes \$2.5 $\mathrm{M}$ in equipment costs. 


\section{RESOURCE PROJECTIONS}

Resource projections for the Institutional Plan provide a description of the budget authority to implement the research programs. The resource tables also indicate actual FY 1992 BA and estimated FY 1993 BA for comparison. These tables include:

- Resource Summaries (page 11-2);

- Secretarial Level Resources (pages 11.3 and 11-4);

- Program Office Resources (pages 11.5 through 11-8);

- Work for Others Resources (page 11-9); and

- Subcontracting and Procurement (Page 11-10).

The FY 1994 estimate is based on FY 1994 DOE budget guidance and assessments by LBL divisions. For fiscal years 1995 and beyond, operating cost projections are in FY 1995 dollars and construction costs are in actualyear doilars (as indicated in the DOE guidance). For FY 1995 to FY 1999, the growth assumptions in program areas as tabulated range from $0 \%$ to $1.5 \%$ per year. These growth assumptions are based on the general direction indicated by DOE program personnel. Specific trend levels were established within each program activity.

The resource projections that follow include all funded and budgeted construction projects, the projected MEL-FS program, the approved Environmental Restoration and Waste Management program funding and construction and operational costs for the Advanced Light Source. The Nuclear Physics Program at the Bevalac ended in FY 1993. The new initiative costs are indicated in Section 4. Proposed construction project costs are provided in Section 9. The distribution of direct and indirect full-time equivalents is integrated with ES\&H staffing planning, but is insufficient for full support of the 5-year ES\&H plan and Corrective Action Plan (see Section 6), which requires additional program support for full implementation of these plans. 
Funding Summary (Fiscal Year Budget Authority, SM)

\begin{tabular}{lrrrrrrrr}
\hline Category & 1992 & 1993 & 1994 & 1995 & 1996 & 1997 & 1998 & 1999 \\
DOE Operating & 180.3 & 173.7 & 183.1 & 192.8 & 195.3 & 194.1 & 195.4 & 198.5 \\
WF() Operating & 37.9 & 42.3 & 52.6 & 48.9 & 45.6 & 44.9 & 43.2 & 43.5 \\
$\quad$ Total Operating & 218.2 & 216.0 & 235.7 & 241.7 & 240.9 & 239.0 & 238.6 & 242.0 \\
Capital Equipment & 34.8 & 32.5 & 45.8 & 36.2 & 33.0 & 29.0 & 26.4 & 26.9 \\
Program Construction & 17.6 & 3.4 & 7.1 & 21.1 & 11.4 & 6.5 & 5.5 & 5.5 \\
Ciencral Purpose Facilities & 11.0 & 4.1 & 5.3 & 6.7 & 15.2 & 32.3 & 33.9 & 27.0 \\
Environmental Restoration & 5.0 & 0.5 & 5.8 & 0.4 & 0.0 & 0.0 & 0.0 & 0.0 \\
Cieneral Plant Projects & 1.2 & 3.4 & 4.0 & 4.5 & 4.5 & 4.5 & 4.5 & 4.5 \\
General Purpose Equipment & 1.7 & 1.9 & 5.0 & 5.5 & 5.5 & 5.5 & 5.5 & 5.5 \\
$\quad$ Total Lab Funding & 289.5 & 261.8 & 308.7 & 316.1 & $\mathbf{3 1 0 . 5}$ & $\mathbf{3 1 6 . 8}$ & 314.4 & 311.4 \\
\hline
\end{tabular}

Personnel Summary (Fiscal Year FTE)

\begin{tabular}{lrrrrrrrr}
\hline Category & 1992 & 1993 & 1994 & 1995 & 1996 & 1997 & 1998 & 1999 \\
\hline DOE Effort & 1579 & 1588 & 1549 & 1560 & 1570 & 1578 & 1587 & 1595 \\
WFO & 274 & 310 & 298 & 293 & 296 & 290 & 279 & 281 \\
$\quad$ Total Direct & 1853 & 1898 & 1846 & 1854 & 1866 & 1868 & 1866 & 1876 \\
Total Indirect & 764 & 783 & 772 & 772 & 772 & 772 & 772 & 772 \\
Total Lab Personnel & 2617 & 2681 & $\mathbf{2 6 1 8}$ & $\mathbf{2 6 2 5}$ & $\mathbf{2 6 3 8}$ & $\mathbf{2 6 4 0}$ & $\mathbf{2 6 3 8}$ & $\mathbf{2 6 4 8}$ \\
\hline
\end{tabular}


Secretarial Office Funding Summary (Fiscal Year Budget Authority, SM)

\begin{tabular}{|c|c|c|c|c|c|c|c|c|}
\hline Office/Program & 1992 & 1993 & 1994 & 1995 & 1996 & 1997 & 1998 & 1999 \\
\hline \multicolumn{9}{|l|}{ Office of Energy Research } \\
\hline Operaling & $: 33.3$ & 130.5 & 131.3 & 136.0 & 137.0 & 138.1 & 139.1 & 140.2 \\
\hline Capital Equipment & 23.0 & 16.7 & 20.8 & 19.4 & 17.6 & 17.7 & 17.8 & 17.7 \\
\hline Construction & 24.8 & 10.0 & 14.4 & 30.3 & 29.1 & 41.3 & 41.9 & 35.0 \\
\hline Total & 181.1 & 157.2 & 166.5 & 185.7 & 183.7 & 197.1 & 198.8 & 192.9 \\
\hline \multicolumn{9}{|c|}{ Energy Efficiency and Renewable Energy } \\
\hline Operating & 15.5 & 17.4 & 22.0 & 23.0 & 23.2 & 23.1 & 23.1 & 23.1 \\
\hline Capital Equipment & 0.7 & 0.7 & 0.8 & 0.8 & 0.8 & 0.8 & 0.8 & 0.8 \\
\hline Tutal & 16.2 & 18.1 & 22.8 & 23.8 & 24.0 & 23.9 & 23.9 & 23.9 \\
\hline \multicolumn{9}{|l|}{ Fossil Energy } \\
\hline Operating & 2.2 & 2.2 & 2.2 & 2.3 & 2.3 & 2.3 & 2.3 & 2.3 \\
\hline Capital Equipment & 0.0 & 0.0 & 0.1 & 0.0 & 0.1 & 0.0 & 0.1 & 0.0 \\
\hline Total & 2.2 & 2.2 & 2.3 & 2.3 & 2.4 & 2.3 & 2.4 & 2.3 \\
\hline \multicolumn{9}{|l|}{ Civilian Waste Management } \\
\hline Operating & 3.4 & 3.2 & 3.3 & 3.5 & 3.5 & 3.5 & 3.5 & 3.5 \\
\hline Capital Equipment & 0.1 & 0.1 & 0.1 & 0.1 & 0.1 & 0.1 & 0.1 & 0.1 \\
\hline Total & 3.5 & 3.3 & 3.4 & 3.6 & 3.6 & 3.6 & 3.6 & 3.6 \\
\hline \multicolumn{9}{|c|}{ Environmental Restoration and Waste Management } \\
\hline Operating & 9.2 & 9.7 & 14.0 & 17.5 & 18.7 & 16.5 & 16.8 & 18.8 \\
\hline Capital Equipment & 0.5 & 0.2 & 0.9 & 0.9 & 0.5 & 0.5 & 0.7 & 1.4 \\
\hline Construction & 5.0 & 0.5 & 5.8 & 0.4 & 0.0 & 0.0 & 0.0 & 0.0 \\
\hline Total & 14.7 & 10.4 & 20.7 & 18.8 & 19.2 & 17.0 & 17.5 & 20.2 \\
\hline \multicolumn{9}{|c|}{ Environment, Health and Safety } \\
\hline Operating & 1.7 & 2.6 & 2.6 & 2.7 & 2.8 & 2.8 & 2.8 & 2.8 \\
\hline Capital Equipment & 0.1 & 0.0 & 0.0 & 0.0 & 0.0 & 0.0 & 0.0 & 0.0 \\
\hline Total & 1.8 & 2.6 & 2.6 & 2.7 & 2.8 & 2.8 & 2.8 & 2.8 \\
\hline \multicolumn{9}{|c|}{ Administration and Human Resource Management } \\
\hline Operating & 0.3 & 0.2 & 0.3 & 0.3 & 0.3 & 0.3 & 0.3 & 0.3 \\
\hline Capital Equipment & 0.0 & 0.0 & 0.0 & 0.0 & 0.0 & 0.0 & 0.0 & 0.0 \\
\hline Construction & 5.0 & 0.9 & 2.0 & 2.0 & 2.0 & 2.0 & 2.0 & 2.0 \\
\hline Total & 5.3 & 1.1 & 2.3 & 2.3 & 2.3 & 2.3 & 2.3 & 2.3 \\
\hline \multicolumn{9}{|c|}{ Domestic \& International Energy Policy } \\
\hline Operating & 1.6 & 1.5 & 2.0 & 2.1 & 2.1 & 2.1 & 2.1 & 2.1 \\
\hline Total & 1.6 & 1.5 & 2.0 & 2.1 & 2.1 & 2.1 & 2.1 & 2.1 \\
\hline \multicolumn{9}{|c|}{ Work for Other DOE Contractors } \\
\hline Operating & 13.1 & 6.4 & 5.4 & 5.4 & 5.4 & 5.4 & 5.4 & 5.4 \\
\hline Capital Equipment & 3.7 & 7.8 & 12.2 & 10.1 & 10.0 & 10.0 & 10.0 & 10.0 \\
\hline Total & 16.8 & 14.2 & 17.6 & 15.5 & 15.4 & 15.4 & 15.4 & 15.4 \\
\hline \multicolumn{9}{|l|}{ Total DOE } \\
\hline Operating & 180.3 & 173.7 & 183.1 & 192.8 & 195.3 & 194.1 & 195.4 & 198.5 \\
\hline Capital Equip. (inc.GPE) & 28.1 & 25.5 & 34.9 & 31.3 & 29.1 & 29.1 & 29.5 & 30.0 \\
\hline Construction (inc. MEL) & 34.8 & 11.4 & 22.2 & 32.7 & 31.1 & 43.3 & 43.9 & 37.0 \\
\hline Total & 243.2 & 210.6 & 240.2 & 256.8 & 255.5 & 266.5 & 268.8 & 265.5 \\
\hline Work for Others & 46.3 & 51.2 & 68.5 & 59.3 & 55.0 & 50.3 & 45.6 & 45.9 \\
\hline Total Lab Funding & 289.5 & 261.8 & 308.7 & 316.1 & 310.5 & 316.8 & 314.4 & 311.4 \\
\hline
\end{tabular}


Personnel By Assistant Secretary Level Office (Fiscal Year FTE)

\begin{tabular}{|c|c|c|c|c|c|c|c|c|}
\hline Office/Program & 1992 & 1993 & 1994 & 1995 & 1996 & 1997 & 1998 & 1999 \\
\hline Office of Energy Research & 1225 & 1195 & 1104 & 1112 & 1120 & 1128 & 1137 & 1145 \\
\hline Energy Efficiency \& Renewable Energy & 138 & 163 & 202 & 204 & 205 & 205 & 205 & 205 \\
\hline Fossil Energy & 18 & 19 & 19 & 19 & 19 & 19 & 19 & 19 \\
\hline Civilian Waste Management & 29 & 29 & 25 & 26 & 26 & 26 & 26 & 26 \\
\hline Environment, Health and Safety & 11 & 14 & 15 & 15 & 16 & 16 & 16 & 16 \\
\hline Environmental Restoration & 39 & 58 & 67 & 67 & 67 & 67 & 67 & 67 \\
\hline Administration \& Human Resource & 5 & 8 & 8 & 8 & 8 & 8 & 8 & 8 \\
\hline Policy, Planning and Analysis & 11 & 12 & 14 & 14 & 14 & 14 & 14 & 14 \\
\hline Other DOE Contractors & 103 & 90 & 95 & 95 & 95 & 95 & 95 & 95 \\
\hline Total DOE & 1579 & 1588 & 1549 & 1560 & 1570 & 1578 & 1587 & 1595 \\
\hline Work for Others & 274 & 310 & 298 & 293 & 296 & 290 & 279 & 281 \\
\hline Total Direct & 1853 & 1898 & 1846 & 1854 & 1866 & 1868 & 1866 & 1876 \\
\hline Total Indirect & 764 & 783 & 772 & 772 & 772 & 772 & 772 & 772 \\
\hline Total Personnel & 2617 & 2681 & 2618 & 2625 & 1638 & 2640 & 2638 & 2648 \\
\hline
\end{tabular}


Office of Energy Research Funding and Personnel (FY BA, \$M)

\begin{tabular}{|c|c|c|c|c|c|c|c|c|}
\hline Office/Program & 1992 & 1993 & 1994 & 1995 & 1996 & 1997 & 1998 & 1999 \\
\hline \multicolumn{9}{|l|}{ AT Fusion Energy } \\
\hline Operating & 7.8 & 7.2 & 6.9 & 7.2 & 7.2 & 7.2 & 7.2 & 7.2 \\
\hline Capital Equipment & 1.3 & 1.4 & 1.0 & 1.0 & 1.0 & 1.0 & 1.0 & 1.0 \\
\hline $\begin{array}{l}\text { Total } \\
\text { Direct FTE }\end{array}$ & $\begin{array}{l}9.1 \\
65\end{array}$ & $\begin{array}{l}8.6 \\
60\end{array}$ & $\begin{array}{l}7.9 \\
52\end{array}$ & $\begin{array}{l}8.2 \\
54\end{array}$ & $\begin{array}{c}8.2 \\
54\end{array}$ & $\begin{array}{c}8.2 \\
54\end{array}$ & $\begin{array}{c}8.2 \\
54\end{array}$ & $\begin{array}{c}8.2 \\
54\end{array}$ \\
\hline \multicolumn{9}{|c|}{ KA High Energy Physics } \\
\hline Operating & 20.6 & 19.7 & 19.7 & 20.6 & 20.9 & 21.2 & 21.5 & 21.9 \\
\hline Capital Equipment & 2.1 & 2.1 & 2.1 & 2.1 & 2.1 & 2.1 & 2.1 & 2.1 \\
\hline $\begin{array}{l}\text { Total } \\
\text { Direct FTE }\end{array}$ & $\begin{array}{l}22.7 \\
164\end{array}$ & $\begin{array}{l}21.8 \\
164\end{array}$ & $\begin{array}{l}\mathbf{2 1 . 8} \\
150\end{array}$ & $\begin{array}{l}\mathbf{2 2 . 7} \\
151\end{array}$ & $\begin{array}{l}\mathbf{2 3 . 0} \\
153\end{array}$ & $\begin{array}{l}\mathbf{2 3 . 3} \\
156\end{array}$ & $\begin{array}{l}\mathbf{2 3 . 6} \\
158\end{array}$ & $\begin{array}{l}\mathbf{2 4 . 0} \\
160\end{array}$ \\
\hline \multicolumn{9}{|l|}{ KB Nuclear Physics } \\
\hline Operating & 32.6 & 25.1 & 22.1 & 21.1 & 21.1 & 21.1 & 21.1 & 21.1 \\
\hline Capital Equipment & 11.5 & 6.6 & 10.7 & 9.3 & 7.5 & 7.6 & 7.7 & 7.6 \\
\hline Construction & 4.1 & 3.7 & 4.4 & 5.0 & 5.0 & 5.0 & 5.0 & 5.0 \\
\hline $\begin{array}{l}\text { Total } \\
\text { Direct FTE }\end{array}$ & $\begin{array}{l}48.2 \\
309\end{array}$ & $\begin{array}{l}35.4 \\
265\end{array}$ & $\begin{array}{l}37.2 \\
230\end{array}$ & $\begin{array}{l}35.4 \\
221\end{array}$ & $\begin{array}{l}\mathbf{3 3 . 6} \\
221\end{array}$ & $\begin{array}{l}\mathbf{3 3 . 7} \\
221\end{array}$ & $\begin{array}{l}33.8 \\
221\end{array}$ & $\begin{array}{l}33.7 \\
221\end{array}$ \\
\hline \multicolumn{9}{|c|}{ KC 02 Materials Sciences } \\
\hline Operating & 35.2 & 37.9 & 39.9 & 42.6 & 42.9 & 43.1 & 43.3 & 43.6 \\
\hline Capital Equipment & 3.8 & 3.5 & 4.5 & 4.5 & 4.5 & 4.5 & 4.5 & 4.5 \\
\hline Construction & 8.0 & 1.6 & 2.5 & 3.0 & 3.0 & 3.0 & 3.0 & 3.0 \\
\hline $\begin{array}{l}\text { Total } \\
\text { Direct FTE }\end{array}$ & $\begin{array}{l}47.0 \\
384\end{array}$ & $\begin{array}{l}43.0 \\
368\end{array}$ & $\begin{array}{l}\mathbf{4 6 . 9} \\
340\end{array}$ & $\begin{array}{l}\mathbf{5 0 . 1} \\
349\end{array}$ & $\begin{array}{l}\mathbf{5 0 . 4} \\
351\end{array}$ & $\begin{array}{l}\mathbf{5 0 . 6} \\
354\end{array}$ & $\begin{array}{l}\mathbf{5 0 . 8} \\
356\end{array}$ & $\begin{array}{l}\mathbf{5 1 . 1} \\
359\end{array}$ \\
\hline \multicolumn{9}{|c|}{ KC 03 Chemical Sciences } \\
\hline Operating & 7.9 & 8.3 & 7.7 & 8.1 & 8.2 & 8.3 & 8.5 & 8.6 \\
\hline Capital Equipment & 1.4 & 2.3 & 1.0 & 1.0 & 1.0 & 1.0 & 1.0 & 1.0 \\
\hline $\begin{array}{l}\text { Total } \\
\text { Direct FTE }\end{array}$ & $\begin{array}{l}9.3 \\
67\end{array}$ & $\begin{array}{l}10.6 \\
64\end{array}$ & $\begin{array}{c}8.7 \\
63\end{array}$ & $\begin{array}{l}9.1 \\
63\end{array}$ & $\begin{array}{r}9.2 \\
64\end{array}$ & $\begin{array}{l}9.3 \\
65\end{array}$ & $\begin{array}{l}9.5 \\
66\end{array}$ & $\begin{array}{l}9.6 \\
67\end{array}$ \\
\hline \multicolumn{9}{|c|}{ KC 04 Engineering, Math and Geosciences } \\
\hline Operating & 2.4 & 2.5 & 2.5 & 2.6 & 2.7 & 2.7 & 2.7 & 2.8 \\
\hline Capital Equipment & 0.1 & 0.1 & 0.1 & 0.1 & 0.1 & 0.1 & 0.1 & 0.1 \\
\hline Total & 2.5 & 2.6 & 2.6 & 2.7 & 2.8 & 2.8 & 2.8 & 2.9 \\
\hline Direct FTE & 24 & 24 & 24 & 24 & 24 & 25 & 25 & 25 \\
\hline \multicolumn{9}{|c|}{ KC 05 Advanced Energy Projects } \\
\hline Operating & 0.5 & 0.5 & 0.6 & 0.6 & 0.6 & 0.6 & 0.7 & 0.7 \\
\hline Capital Equipment & 0.1 & 0.0 & 0.1 & 0.1 & 0.1 & 0.1 & 0.1 & 0.1 \\
\hline Total & 0.6 & 0.5 & 0.7 & 0.7 & 0.7 & 0.7 & 0.8 & 0.8 \\
\hline Direct FTE & 7 & 5 & 5 & 5 & 5 & 5 & 5 & 5 \\
\hline \multicolumn{9}{|c|}{ KC 06 Energy Biosciences } \\
\hline Operating & 1.1 & 1.1 & 1.1 & 1.2 & 1.2 & 1.2 & 1.2 & 1.2 \\
\hline Capital Equipment & 0.1 & 0.1 & 0.1 & 0.1 & 0.1 & 0.1 & 0.1 & 0.1 \\
\hline Total & 1.2 & 1.2 & 1.2 & 1.3 & 1.3 & 1.3 & 1.3 & 1.3 \\
\hline Direct FTE & 8 & 8 & 8 & 8 & 8 & 8 & 8 & 8 \\
\hline \multicolumn{9}{|c|}{ KC 07 Applied Math Sciences } \\
\hline Operating & 3.1 & 3.3 & 3.3 & 3.5 & 3.5 & 3.6 & 3.6 & 3.7 \\
\hline Capital Equipment & 0.1 & 0.1 & 0.1 & 0.1 & 0.1 & 0.1 & 0.1 & 0.1 \\
\hline Total & 3.2 & 3.4 & 3.4 & 3.6 & 3.6 & 3.7 & 3.7 & 3.8 \\
\hline Direct FTE & 21 & 23 & 23 & 23 & 23 & 24 & 24 & 25 \\
\hline
\end{tabular}


Office of Energy Research Funding and Personnel (FY BA, \$M) (continued).

\begin{tabular}{|c|c|c|c|c|c|c|c|c|}
\hline Office/Program & 1992 & 1993 & 1994 & 1995 & 1996 & 1997 & 1998 & 1999 \\
\hline \multicolumn{9}{|c|}{ KC Basic Energy Sciences (Total) } \\
\hline Operating & 50.2 & 53.6 & 55.1 & 58.5 & 59.1 & 59.5 & 60.0 & 60.5 \\
\hline Capital Equipment & 5.6 & 6.1 & 5.9 & 5.9 & 5.9 & 5.9 & 5.9 & 5.9 \\
\hline Construction & 8.0 & 1.6 & 2.5 & 3.0 & 3.0 & 3.0 & 3.0 & 3.0 \\
\hline Total & 63.8 & 61.3 & 63.5 & 67.4 & 68.0 & 68.4 & 68.9 & 69.4 \\
\hline Direct FTE & 511 & 492 & 463 & 472 & 476 & 480 & 485 & 489 \\
\hline \multicolumn{9}{|c|}{ KG General Purpose Facilities Revitalization } \\
\hline Operating & 0.0 & 0.0 & 0.0 & 0.0 & 0.0 & 0.0 & 0.0 & 0.0 \\
\hline Capital Equipment & 0.0 & 0.0 & 0.0 & 0.0 & 0.0 & 0.0 & 0.0 & 0.0 \\
\hline Construction & 11.0 & 4.1 & 5.3 & 6.7 & 15.2 & 32.3 & 33.9 & 27.0 \\
\hline Total & 11.0 & 4.1 & 5.3 & 6.7 & 15.2 & 32.3 & 33.9 & 27.0 \\
\hline Direct FTE & 12 & 20 & 17 & 18 & 18 & 18 & 18 & 18 \\
\hline \multicolumn{9}{|c|}{ KP Biological and Environmental Research } \\
\hline Operating & 17.5 & 20.6 & 20.6 & 21.5 & 21.8 & 22.0 & 22.2 & 22.4 \\
\hline Capital Equipment & 2.1 & 0.5 & 1.1 & 1.1 & 1.1 & 1.1 & 1.1 & 1.1 \\
\hline Construction & 1.7 & 0.6 & 2.2 & 15.6 & 5.9 & 1.0 & 0.0 & 0.0 \\
\hline Total & 21.3 & 21.7 & 23.9 & 38.2 & 28.8 & 24.1 & 23.3 & 23.5 \\
\hline Direct FTE & 147 & 170 & 160 & 163 & 164 & 166 & 167 & 169 \\
\hline \multicolumn{9}{|c|}{ KS Superconducting Super Collider } \\
\hline Operating & 0.0 & 0.0 & 0.0 & 0.0 & 0.0 & 0.0 & 0.0 & 0.0 \\
\hline Capital Equipment & 0.4 & 0.0 & 0.0 & 0.0 & 0.0 & 0.0 & 0.0 & 0.0 \\
\hline Construction & 0.0 & 0.0 & 0.0 & 0.0 & 0.0 & 0.0 & 0.0 & 0.0 \\
\hline Total & 0.4 & 0.0 & 0.0 & 0.0 & 0.0 & 0.0 & 0.0 & 0.0 \\
\hline Direct FTE & 5 & 5 & 0 & 0 & 0 & 0 & 0 & 0 \\
\hline \multicolumn{9}{|c|}{$\mathrm{KT} / \mathrm{KV}$ University and Science Education } \\
\hline Operating & 2.5 & 2.5 & 2.9 & 3.0 & 3.0 & 3.0 & 3.0 & 3.0 \\
\hline Capital Equipment & 0.0 & 0.0 & 0.0 & 0.0 & 0.0 & 0.0 & 0.0 & 0.0 \\
\hline Construction & 0.0 & 0.0 & 0.0 & 0.0 & 0.0 & 0.0 & 0.0 & 0.0 \\
\hline Total & 2.5 & 2.5 & 2.9 & 3.0 & 3.0 & 3.0 & 3.0 & 3.0 \\
\hline Direct FTE & 9 & 9 & 11 & 13 & 13 & 13 & 13 & 13 \\
\hline \multicolumn{9}{|c|}{ KU Laboratory Technology Transfer } \\
\hline Operating & 2.1 & 1.8 & 4.0 & 4.0 & 4.0 & 4.0 & 4.0 & 4.0 \\
\hline Capital Equipment & 0.0 & 0.0 & 0.0 & 0.0 & 0.0 & 0.0 & 0.0 & 0.0 \\
\hline Construction & 0.0 & 0.0 & 0.0 & 0.0 & 0.0 & 0.0 & 0.0 & 0.0 \\
\hline Total & 2.1 & 1.8 & 4.0 & 4.0 & 4.0 & 4.0 & 4.0 & 4.0 \\
\hline Direct FTE & 3 & 10 & 20 & 20 & 20 & 20 & 20 & 20 \\
\hline \multicolumn{9}{|l|}{ Total OER } \\
\hline Operating & 133.3 & 130.3 & 131.3 & 136.0 & 137.0 & 138.1 & 139.1 & 140.2 \\
\hline Capital Equipment & 23.0 & 16.7 & 20.8 & 19.4 & 17.6 & 17.7 & 17.8 & 17.7 \\
\hline Construction & 24.8 & 10.0 & 14.4 & 30.3 & 29.1 & 31.3 & 41.9 & 35.0 \\
\hline Total & 181.1 & 157.2 & 166.5 & 185.7 & 183.7 & 197.1 & 198.8 & 192.9 \\
\hline Direct FTE & 1225 & 1195 & 1104 & 1112 & 1120 & 1128 & 1137 & 1145 \\
\hline
\end{tabular}


Energy Efficiency and Renewable Energy Funding and Personnel (FY BA, \$M)

\begin{tabular}{|c|c|c|c|c|c|c|c|c|}
\hline Office/Program & 1992 & 1993 & 1994 & 1995 & 1996 & 1997 & 1998 & 1999 \\
\hline \multicolumn{9}{|c|}{ AK Electric Energy Systems } \\
\hline Operating & 0.2 & 0.2 & 0.2 & 0.2 & 0.2 & 0.2 & 0.2 & 0.2 \\
\hline Capital Equipment & 0.0 & 0.0 & 0.0 & 0.0 & 0.0 & 0.0 & 0.0 & 0.0 \\
\hline Total & 0.2 & 0.2 & 0.2 & 0.2 & 0.2 & 0.2 & 0.2 & 0.2 \\
\hline Direct FTE & 1 & 1 & 1 & 1 & 1 & 1 & 1 & 1 \\
\hline \multicolumn{9}{|l|}{ AM Geothermal Energy } \\
\hline Operating & 1.4 & 0.8 & 0.8 & 0.9 & 1.0 & 1.0 & 1.0 & 1.0 \\
\hline Capital Equipment & 0.1 & 0.1 & 0.1 & 0.1 & 0.1 & 0.1 & 0.1 & 0.1 \\
\hline Total & 1.5 & 0.9 & 0.9 & 1.0 & 1.1 & 1.1 & 1.1 & 1.1 \\
\hline Direct FTE & 10 & 9 & 9 & 9 & 10 & 10 & 10 & 10 \\
\hline \multicolumn{9}{|l|}{ EC Building Sector } \\
\hline Operating & 8.8 & 10.2 & 14.6 & 15.3 & 15.3 & 15.3 & 15.3 & 15.3 \\
\hline Capital Equipment & 0.5 & 0.5 & 0.6 & 0.6 & 0.6 & 0.6 & 0.6 & 0.6 \\
\hline Total & 9.3 & 10.5 & 15.2 & 15.9 & 15.9 & 15.9 & 15.9 & 15.9 \\
\hline Direct FTE & 89 & 105 & 145 & 146 & 146 & 146 & 146 & 146 \\
\hline \multicolumn{9}{|l|}{ ED Industrial Sector } \\
\hline Operating & 1.3 & 1.2 & 1.2 & 1.3 & 1.3 & 1.3 & 1.3 & 1.3 \\
\hline Capital Equipment & 0.1 & 0.0 & 0.0 & 0.0 & 0.0 & 0.0 & 0.0 & 0.0 \\
\hline Total & 1.4 & 1.2 & 1.2 & 1.3 & 1.3 & 1.3 & 1.3 & 1.3 \\
\hline Direct FTE & 10 & 14 & 13 & 14 & 14 & 14 & 14 & 14 \\
\hline \multicolumn{9}{|l|}{ EE Transportation Sector } \\
\hline Operating & 3.0 & 3.9 & 3.9 & 4.1 & 4.1 & 4.1 & 4.1 & 4.1 \\
\hline Capital Equipment & 0.0 & 0.1 & 0.1 & 0.1 & 0.1 & 0.1 & 0.1 & 0.1 \\
\hline Total & 3.0 & 4.0 & 4.0 & 4.2 & 4.2 & 4.2 & 4.2 & 4.2 \\
\hline Direct FTE & 23 & 25 & 25 & 24 & 24 & 24 & 24 & 24 \\
\hline \multicolumn{9}{|l|}{ EF State/Local Programs } \\
\hline Operating & 0.0 & 0.1 & 0.2 & 0.2 & 0.2 & 0.2 & 0.2 & 0.2 \\
\hline Capital Equipment & 0.0 & 0.0 & 0.0 & 0.0 & 0.0 & 0.0 & 0.0 & 0.0 \\
\hline Total & 0.0 & 0.1 & 0.2 & 0.2 & 0.2 & 0.2 & 0.2 & 0.2 \\
\hline Direct FTE & 0 & 1 & 1 & 1 & 1 & 1 & 1 & 1 \\
\hline \multicolumn{9}{|l|}{ EK Utility Sector } \\
\hline Operating & 0.8 & 1.0 & 1.1 & 1.1 & 1.1 & 1.1 & 1.1 & 1.1 \\
\hline Capital Equipment & 0.0 & 0.0 & 0.0 & 0.0 & 0.0 & 0.0 & 0.0 & 0.0 \\
\hline Total & 0.8 & 1.0 & 1.1 & 1.1 & 1.1 & 1.1 & 1.1 & 1.1 \\
\hline Direct FTE & 5 & 8 & 9 & 9 & 9 & 9 & 9 & 9 \\
\hline \multicolumn{9}{|c|}{ Total Energy Efficiency and Renewable Energy } \\
\hline Operating & 15.5 & 17.4 & 22.0 & 23.0 & 23.2 & 23.1 & 23.1 & 23.1 \\
\hline Capital Equipment & 0.7 & 0.7 & 0.8 & 0.8 & 0.8 & 0.8 & 0.8 & 0.8 \\
\hline Total & 16.2 & 18.1 & 22.8 & 23.8 & 24.0 & 23.9 & 23.9 & 23.9 \\
\hline Direct FTE & 138 & 163 & 202 & 204 & 205 & 205 & 205 & 205 \\
\hline
\end{tabular}


Fossil Fuel and Other DOE Program Funding and Personnel (FY BA, \$M)

\begin{tabular}{|c|c|c|c|c|c|c|c|c|}
\hline Office/Program & 1992 & 1993 & 1994 & 1995 & 1996 & 1997 & 1998 & 1999 \\
\hline \multicolumn{9}{|l|}{ AA Coal } \\
\hline Operating & 1.0 & 0.9 & 0.9 & 0.9 & 0.9 & 0.9 & 0.9 & 0.9 \\
\hline Capital Equipment & 0.0 & 0.0 & 0.1 & 0.0 & 0.1 & 0.0 & 0.1 & 0.0 \\
\hline Total & 1.0 & 0.9 & 1.0 & 0.9 & 1.0 & 0.9 & 1.0 & 0.9 \\
\hline Direct FTE & 9 & 7 & 7 & 7 & 7 & 7 & 7 & 7 \\
\hline \multicolumn{9}{|l|}{ AB Gas } \\
\hline Operating & 0.3 & 0.5 & 0.5 & 0.5 & 0.5 & 0.5 & 0.5 & 0.5 \\
\hline Total & 0.3 & 0.5 & 0.5 & 0.5 & 0.5 & 0.5 & 0.5 & 0.5 \\
\hline Direct FTE & 2 & 5 & 5 & 5 & 5 & 5 & 5 & 5 \\
\hline \multicolumn{9}{|l|}{ AC Petroleum } \\
\hline Operating & 0.9 & 0.8 & 0.8 & 0.8 & 0.8 & 0.8 & 0.8 & 0.8 \\
\hline Total & 0.9 & 0.8 & 0.8 & 0.8 & 0.8 & 0.8 & 0.8 & 0.8 \\
\hline Direct FTE & 7 & 7 & 7 & 7 & 7 & 7 & 7 & 7 \\
\hline \multicolumn{9}{|l|}{ Total Fossil } \\
\hline Operating & 2.2 & 2.2 & 2.2 & 2.3 & 2.3 & 2.3 & 2.3 & 2.3 \\
\hline Capital Equipment & 0.0 & 0.0 & 0.1 & 0.0 & 0.1 & 0.0 & 0.1 & 0.0 \\
\hline Total & 2.2 & 2.2 & 2.3 & 2.3 & 2.4 & 2.3 & 2.4 & 2.3 \\
\hline Direct FTE & 18 & 19 & 19 & 19 & 19 & 19 & 19 & 19 \\
\hline \multicolumn{9}{|c|}{ DB Civilian Waste Management } \\
\hline Operating & 3.4 & 3.2 & 3.2 & 3.5 & 3.5 & 3.5 & 3.5 & 3.5 \\
\hline Capital Equipment & 0.1 & 0.1 & 0.1 & 0.1 & 0.1 & 0.1 & 0.1 & 0.1 \\
\hline Total & 3.5 & 3.3 & 3.4 & 3.6 & 3.6 & 3.6 & 3.6 & 3.6 \\
\hline Direct FTE & 29 & 29 & 25 & 26 & 26 & 26 & 26 & 26 \\
\hline \multicolumn{9}{|c|}{ EX/EW Environmental Restoration and Waste Management } \\
\hline Operating & 9.2 & 9.7 & 14.0 & 17.5 & 18.7 & 16.5 & 16.8 & 18.8 \\
\hline Capital Equipment & 0.5 & 0.2 & 0.9 & 0.9 & 0.5 & 0.5 & 0.7 & 1.4 \\
\hline Construction & 5.0 & 0.5 & 5.8 & 0.4 & 0.0 & 0.0 & 0.0 & 0.0 \\
\hline Total & 14.7 & 10.4 & 20.7 & 18.8 & 19.2 & 17.0 & 17.5 & 20.2 \\
\hline Direct FTE & 39 & 58 & 67 & 67 & 67 & 67 & 67 & 67 \\
\hline \multicolumn{9}{|c|}{ HA Environment, Safety \& Health } \\
\hline Operating & 1.7 & 2.6 & 2.6 & 2.7 & 2.8 & 2.8 & 2.8 & 2.8 \\
\hline Capital Equipment & 0.1 & 0.0 & 0.0 & 0.0 & 0.0 & 0.0 & 0.0 & 0.0 \\
\hline Total & 1.8 & 2.6 & 2.6 & 2.7 & 2.8 & 2.8 & 2.8 & 2.8 \\
\hline Direct FTE & 11 & 14 & 15 & 15 & 16 & 16 & 16 & 16 \\
\hline \multicolumn{9}{|c|}{ WB Administration and Human Resource Management } \\
\hline Operating & 0.3 & 0.2 & 0.3 & 0.3 & 0.3 & 0.3 & 0.3 & 0.3 \\
\hline Construction & 5.0 & 0.9 & 2.0 & 2.0 & 2.0 & 2.0 & 2.0 & 2.0 \\
\hline Total & 5.3 & 1.1 & 2.3 & 2.3 & 2.3 & 2.3 & 2.3 & 2.3 \\
\hline Direct FTE & 5 & 8 & 8 & 8 & 8 & 8 & 8 & 8 \\
\hline \multicolumn{9}{|c|}{ PE Policy, Planning and Analysis } \\
\hline Operating & 1.6 & 1.5 & 2.0 & 2.1 & 2.1 & 2.1 & 2.1 & 2.1 \\
\hline Total & 1.6 & 1.5 & 2.0 & 2.1 & 2.1 & 2.1 & 2.1 & 2.1 \\
\hline Direct FTE & 11 & 12 & 14 & 14 & 14 & 14 & 14 & 14 \\
\hline \multicolumn{9}{|c|}{ Work for Other DOE Contractors } \\
\hline Operating & 13.1 & 6.4 & 5.4 & 5.4 & 5.4 & 5.4 & 5.4 & 5.4 \\
\hline Capital Equipment & 3.7 & 7.8 & 12.2 & 10.1 & 10.0 & 10.0 & 10.0 & 10.0 \\
\hline Total & 16.8 & 14.2 & 17.6 & 15.5 & 15.4 & 15.4 & 15.4 & 15.4 \\
\hline Direct FTE & 103 & 90 & 95 & 95 & 95 & 95 & 95 & 95 \\
\hline
\end{tabular}


Work for Others Funding and Personnel (FY BA, \$M)

\begin{tabular}{|c|c|c|c|c|c|c|c|c|}
\hline Office/Program & 1992 & 1993 & 1994 & 1995 & 1996 & 1997 & 1998 & 1999 \\
\hline \multicolumn{9}{|c|}{ Other Federal Agencies } \\
\hline AID & 0.3 & 0.1 & 0.1 & 0.0 & 0.0 & 0.0 & 0.0 & 0.0 \\
\hline Direct FTE & 2 & 1 & 1 & 0 & 0 & 0 & 0 & 0 \\
\hline Defense & 12.8 & 12.2 & 30.0 & 20.0 & 15.0 & 10.0 & 5.0 & 5.0 \\
\hline Direct FTE & 32 & 40 & 40 & 40 & 40 & 32 & 20 & 20 \\
\hline EPA & 2.0 & 1.3 & 1.6 & 1.8 & 1.8 & 1.8 & 1.8 & 1.8 \\
\hline Direct FTE & 12 & 11 & 13 & 14 & 14 & 14 & 14 & 14 \\
\hline DOI & 0.7 & 0.6 & 0.7 & 0.7 & 0.7 & 0.7 & 0.7 & 0.7 \\
\hline Direct FTE & 8 & 7 & 8 & 7 & 7 & 7 & 7 & 7 \\
\hline NASA & 3.2 & 3.2 & 2.7 & 2.6 & 2.6 & 2.6 & 2.6 & 2.6 \\
\hline Direct FTE & 19 & 19 & 15 & 14 & 14 & 14 & 14 & 14 \\
\hline $\mathrm{NIH}$ & 14.3 & 15.9 & 16.9 & 17.8 & 18.0 & 18.1 & 18.2 & 18.3 \\
\hline Direct FTE & 106 & 111 & 114 & 116 & 117 & 118 & 119 & 119 \\
\hline Other & 1.0 & 0.5 & 0.7 & 0.8 & 1.3 & 1.5 & 1.7 & 1.9 \\
\hline Direct FTE & 1 & 2 & 3 & 3 & 5 & 6 & 6 & 7 \\
\hline \multicolumn{9}{|c|}{ Total Other Federal Agencies } \\
\hline Operating & 25.9 & 25.5 & 37.2 & 33.7 & 30.4 & 29.7 & 28.0 & 28.3 \\
\hline Capital Equipment & 8.4 & 8.3 & 15.5 & 10.0 & 9.0 & 5.0 & 2.0 & 2.0 \\
\hline Total & 34.3 & 33.8 & 52.7 & 43.7 & 39.4 & 34.7 & 30.0 & 30.3 \\
\hline Direct FTE & 180 & 191 & 193 & 194 & 198 & 191 & 180 & 182 \\
\hline \multicolumn{9}{|l|}{ State/Private } \\
\hline Operating & 12.0 & 16.8 & 15.4 & 15.2 & 15.2 & 15.2 & 15.2 & 15.2 \\
\hline Capital Equipment & 0.0 & 0.6 & 0.4 & 0.4 & 0.4 & 0.4 & 0.4 & 0.4 \\
\hline Total & 12.0 & 17.4 & 15.8 & 15.6 & 15.6 & 15.6 & 15.6 & 15.6 \\
\hline Direct FTE & 94 & 119 & 104 & 99 & 99 & 99 & 99 & 99 \\
\hline \multicolumn{9}{|l|}{ Total Work for Others } \\
\hline Operating & 37.9 & 42.3 & 52.6 & 48.9 & 45.6 & 44.9 & 43.2 & 43.5 \\
\hline Capital Equipment & 8.4 & 8.9 & 15.9 & 10.4 & 9.4 & 5.4 & 2.4 & 2.4 \\
\hline Total & 46.3 & 51.2 & 68.5 & 59.3 & 55.0 & 50.3 & 45.6 & 45.9 \\
\hline Direct FTE & 274 & 310 & 298 & 293 & 296 & 290 & 279 & 281 \\
\hline
\end{tabular}


Subcontracting and Procurement (\$M)

\begin{tabular}{lccc}
\hline Recipient & FY 1992 & FY 1993 (Projected) & FY 1994 (Projected) \\
\hline Universities & 10.0 & 8.0 & 9.0 \\
All Other & 80.0 & 69.0 & 72.0 \\
Other DOE & 2.0 & 1.0 & 1.0 \\
Total & $\mathbf{9 2 . 0}$ & $\mathbf{7 8 . 0}$ & $\mathbf{8 2 . 0}$ \\
\hline
\end{tabular}

Procurement from Disadvantaged, Women Owned, and All Small Businesses

\begin{tabular}{lcc}
\hline Business & FY 1992 & FY 1993 \\
Category & $\$ M(\%)$ & $\$ M(\%)$ \\
\hline Disadvantaged & $6.4(8)$ & $7.0(9)$ \\
Women Owned & $4.0(5)$ & $3.9(5)$ \\
All Small & $36.0(45)$ & $35.8(46)$ \\
\hline
\end{tabular}




\section{ACKNOWLEDGMENTS}

Institutional planning at LBL is conducted as an annual management activity based on technical information contributed by the Laboratory's Division Directors (see organization chart, Section 2). Preparation of reporting documents is coordinated through the Office for Planning and Development. Divisional staff coordinating information and assisting in preparation include:

Accelerator and Fusion Research

Chemical Sciences

Earth Sciences

Energy and Environment

Engineering

Environment, Health and Safety

Information and Computing Sciences

Life Sciences

Materials Sciences

Nuclear Science

Physics

Structural Biology
Joseph T. Chew Linda R. Maio

Karl R. Olson

Donald F. Grether Mary M. Clary

Anil V. Moré

David F. Stevens

David E. Gilbert Mark D. Alper Janis M. Dairiki

Pauline L. Fong

James C. Bartholomew

Robert K. Johnson and G. Douglas Vaughan of the Deputy Director's Office provided review and comments, and scientific program leaders contributed to specific sections of the plan through division offices. In addition, elements of the documents are developed in conjunction with responsible support program administrators:

Assurance and Assessment James F. Koonce/Jonathan W. Stanley Budget and Resource Planning

Educational Programs

Facilities Planning Laboratory Directed R\&D

Planning and Development

Technology Transfer

Workforce Diversity Phillip D. Phythian/Robert B. Shuey Roland J. Otto Laura J. Chen/Richard M. McClure Todd Hansen Pamela J. Patterson

Cheryl A. Fragiadakis/Bruce S. Davies Harry Reed/Christine T. Jue

Correspondence regarding the Institutional Plan can be directed to:

Michael A. Chartock

Office for Planning and Development

Lawrence Berkeley Laboratory, MS 50A-4112

University of California

Berkeley, California 94720

(415) 486-6669; (Fax) 486-6866

MAChartock@lbl.gov 


\section{ACRONYMS AND ABBREVIATIONS}

\begin{tabular}{|c|c|}
\hline AA & Affirmative Action \\
\hline ADA & Americans with Disabilities Act \\
\hline ADEPT & Assisting Development Practices and Technology \\
\hline AECR & Advanced Electron Cyclotron Resonance \\
\hline AEDOT & Advanced Energy Design and Operation Technologies \\
\hline AGMUS & Ana G. Méndez University System \\
\hline ALS & Advanced Light Source \\
\hline ANL & Argonne National Laboratory \\
\hline ARPA & Advanced Research Projects Agency \\
\hline BA & Budget Authority \\
\hline BASTEC & Bay Area Science and Technology Education Collaboration \\
\hline BES & Basic Energy Sciences (Office) \\
\hline BESAC & Basic Energy Sciences Advisory Committee \\
\hline BEST & Bioremediation Education, Science and Technology \\
\hline $\mathrm{BGO}$ & bismuth germanate \\
\hline $\mathrm{BNL}$ & Brookhaven National Laboratory \\
\hline CAD & computer-aided design \\
\hline CAM & Center for Advanced Materials \\
\hline CAMP & Capital Asset Management Plan \\
\hline CCD & charge-coupled device \\
\hline CDF & Collider Detector Facility at Fermilab \\
\hline CDRL & Chemical Dynamics Research Laboratory \\
\hline CEHR & Committee on Education and Human Resources \\
\hline CEQA & California Environmental Quality Act \\
\hline CERCLA & $\begin{array}{l}\text { Comprehensive Environmental Response, Compensation } \\
\text { and Liability Act }\end{array}$ \\
\hline CERN & European Organization for Nuclear Research \\
\hline CIEE & California Institute for Energy Efficiency \\
\hline $\mathrm{CP}$ & charge parity \\
\hline CRADAs & Cooperative Research and Development Agreements \\
\hline CSD & Chemical Sciences Division \\
\hline CSEE & Center for Science and Engineering Education \\
\hline CXRO & Center for X-ray Optics \\
\hline $\mathrm{D} \& \mathrm{D}$ & Decontamination and Decommissioning \\
\hline DLS & Dilepton Spectrometer \\
\hline DOD & U.S. Department of Defense \\
\hline DOE & U.S. Department of Energy \\
\hline DOE/SF & DOE Operations Office, San Francisco (recently changed) \\
\hline DOI & U.S. Department of Interior \\
\hline EAP & Employee Assistance Program \\
\hline EEO & equal employment opportunity \\
\hline EH\&S & Environment, Health and Safety Division (LBL) \\
\hline EM & Environmental Management \\
\hline EPA & U.S. Environmental Protection Agency \\
\hline EPRI & Electric Power Research Institute \\
\hline ER & Office of Energy Research (see also OER) \\
\hline ER-LTT & DOE Energy Research Laboratory Technology Transfer \\
\hline ERVN & ER Videoconference Network \\
\hline ERWM & Environmental Restoration and Waste Management \\
\hline ES\&H & environment, safety, and health \\
\hline
\end{tabular}




\begin{tabular}{|c|c|}
\hline EUV & extreme ultraviolet \\
\hline f.c.c. & face centered cubic \\
\hline FTE & full-time equivalent \\
\hline FY & fiscal year \\
\hline GOSIP & Government Open Systems Interconnection Profile \\
\hline GPE & General Purpose Equipment \\
\hline GPP & General Plant Projects \\
\hline GRI & Gas Research Institute \\
\hline gsf & gross square feet \\
\hline gsm & gross square meters \\
\hline HIFAR & Heavy-Ion Fusion Accelerator Research \\
\hline $\mathrm{HOU}$ & Hands-On Universe \\
\hline HVAC & heating, ventilating, and air-conditioning \\
\hline IC & integrated circuit \\
\hline ICS & Integrated Communication System \\
\hline ILSE & Induction Linac Systems Experiments \\
\hline IRM & information resources management \\
\hline ISL & IsoSpin Laboratory \\
\hline ITER & International Thermonuclear Experimental Reactor \\
\hline ISU & Jackson State University \\
\hline JWS & Joint Work Statement \\
\hline KEK & National Laboratory for High Energy Physics (Japan) \\
\hline LBL & Lawrence Berkeley Laboratory \\
\hline LDRD & Laboratory Directed Research and Development Program \\
\hline LHS & Lawrence Hall of Science \\
\hline LIMS & Laboratory Information Management System \\
\hline LLNL & Lawrence Livermore National Laboratory \\
\hline MAERC & Minority Access to Energy Research Careers \\
\hline MEL-FS & Multiprogram Energy Laboratory Facilities Support \\
\hline Mgsf & million gross square feet \\
\hline MMM & Magnetic Materials Microscope \\
\hline NASA & National Aeronautics and Space Administration \\
\hline NCEM & National Center for Electron Microscopy \\
\hline NDN & Nuclear Data Network \\
\hline NEPA & National Environmental Policy Act \\
\hline NESHAPS & National Emissions Standards Hazards Air Pollutants \\
\hline NIH & National Institutes of Health \\
\hline NMR & nuclear magnetic resonance \\
\hline NPDES & National Pollution Discharge Elimination System \\
\hline NSLS & National Synchrotron Light Source \\
\hline OAP & Operating and Assurance Program \\
\hline OBES & Office of Basic Energy Science \\
\hline OER & Office of Energy Research (see also ER) \\
\hline OFA & other federal agencies \\
\hline OHER & Office of Health and Environmental Research \\
\hline OPD & Office for Planning and Development \\
\hline ORNL & Oak Ridge National Laboratory \\
\hline OSHA & Occupational Safety and Health \\
\hline PAREP & Populations At Risk to Environmental Pollution \\
\hline PEP & Positron Electron Project \\
\hline PETE & Partnership for Environmental Technology Education \\
\hline QA & Quality Assurance \\
\hline$R \& D$ & research and development \\
\hline
\end{tabular}




$\begin{array}{ll}\text { RCRA } & \text { Resource Conservation and Recovery Act } \\ \text { RHIC } & \text { Relativistic Heavy-lon Collider } \\ \text { SDC } & \text { Solenoidal Detector Collaboration } \\ \text { SEABA } & \text { Science Education Academy of the Bay Area } \\ \text { SERS } & \text { Science and Engineering Research Semester } \\ \text { Si } & \text { International System of Units } \\ \text { SLAC } & \text { Stanford Linear Accelerator Center } \\ \text { SLC } & \text { Stanford Linear Collider } \\ \text { SNL } & \text { Sandia National Laboratories } \\ \text { SNO } & \text { Sudbury Neutrino Observatory } \\ \text { SPS } & \text { Super Proton Synchrotron } \\ \text { SQUID } & \text { Superconducting QUantum Interference Device } \\ \text { SSC } & \text { Superconducting Super Collider } \\ \text { STAR } & \text { Solenoidal Tracker at RHIC } \\ \text { SXR } & \text { soft x-ray } \\ \text { TEC } & \text { total estimated cost } \\ \text { TPC } & \text { Time Projection Chamber } \\ \text { TTD } & \text { Technology Transfer Department } \\ \text { UC } & \text { University of California } \\ \text { UCLALEAP } & \text { University of California Los Angeles/Leadership Education } \\ & \text { for Asian Pacifics } \\ \text { UCOP } & \text { University of California, Office of the President } \\ \text { UV } & \text { ultraviolet } \\ \text { VUV } & \text { vacuum ultraviolet } \\ \text { VLSI } & \text { very large scale integration } \\ \text { WFO } & \text { Work for Others }\end{array}$



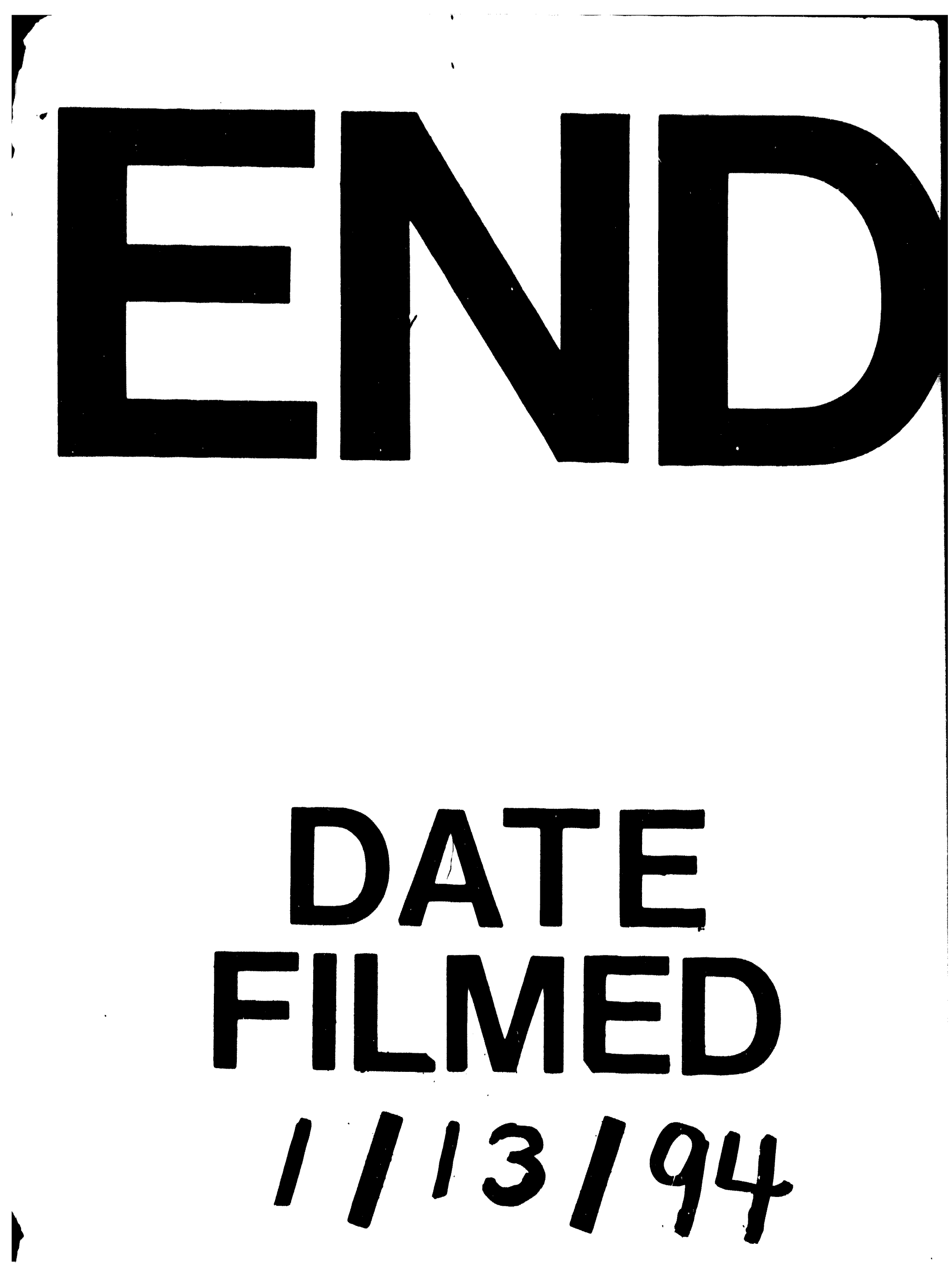
